\title{
Theoretical \& Experimental Studies of Elementary Physics
}

\author{
Award: DE-FG02-91ER40685 \\ University of Rochester \\ Department of Physics and Astronomy \\ FINAL REPORT \\ $11 / 1 / 1991-7 / 15 / 2012$
}

Date submitted: September 28, 2012

Principal Investigators:

Arie Bodek

Ashok Das

Regina Demina

Thomas Ferbel

C. Richard Hagen

Steven Manly

Adrian Melissinos

Kevin McFarland

Susumu Okubo

Stephen Olsen

Lynne Orr

Sarada Rajeev

Paul Slattery

Paul Tipton

Submitted to: DOE/Office of Science Program Office: Office of High Energy Physics

Current Spokesperson for the Principal Investigators:

Kevin McFarland (kevin@rochester.edu (585-275-7076))

University of Rochester

Office of Research and Project Administration

River Campus, Hylan Bldg.

Rochester, NY 14627-0140 


\section{Executive Summary}

High energy physics has been one of the signature research programs at the University of Rochester for over 60 years. The group has made leading contributions to experimental discoveries at accelerators and in cosmic rays and has played major roles in developing the theoretical framework that gives us our "standard model" of fundamental interactions today. This award from the Department of Energy funded a major portion of that research for more than 20 years. During this time, highlights of the supported work included the discovery of the top quark at the Fermilab Tevatron, the completion of a broad program of physics measurements that verified the electroweak unified theory, the measurement of three generations of neutrino flavor oscillations, and the first observation of a "Higgs like" boson at the Large Hadron Collider. The work has resulted in more than 2000 publications over the period of the grant.

The principal investigators supported on this grant have been recognized as leaders in the field of elementary particle physics by their peers through numerous awards and leadership positions. Most notable among them is the APS W.K.H. Panofsky Prize awarded to Arie Bodek in 2004, the J.J. Sakurai Prizes awarded to Susumu Okubo and C. Richard Hagen in 2005 and 2010, respectively, the Wigner medal awarded to Susumu Okubo in 2006, and five principal investigators (Das, Demina, McFarland, Orr, Tipton) who received Department of Energy Outstanding Junior Investigator awards during the period of this grant.

The University of Rochester Department of Physics and Astronomy, which houses the research group, provides primary salary support for the faculty and has waived most tuition costs for graduate students during the period of this grant. The group also benefits significantly from technical support and infrastructure available at the University which supports the work.

The research work of the group has provided educational opportunities for graduate students, undergraduate students and high school students and teachers. Seventy-two graduate students received a Ph.D. in physics for research supported by this grant. 


\section{Introduction}

The University of Rochester Department of Physics and Astronomy has remained committed to its historic strength in high energy physics during the period of this award. As faculty on the grant have retired or left the University, we have continued to recruit in both theoretical and experimental particle physics to maintain a strong group.

The group has completed a large number of experimental and theoretical research programs during the period of this grant. The remainder of this report contains brief summaries of the major activities carried out by the principal investigators during the period of this grant.

\section{Contents}

1 CDF (Bodek, McFarland, Tipton) 5

$\begin{array}{lll}2 & \text { D0 (Demina, Ferbel, Slattery) } & 6\end{array}$

3 CMS (Bodek, Demina, Slattery, Tipton) 6

\begin{tabular}{lll}
\hline 4 & E706 (Ferbel, Slattery) & 7
\end{tabular}

$\begin{array}{llr}5 \text { ATLAS (Slatterv) } & 8\end{array}$

\begin{tabular}{lll}
\hline 6 & AMY (Bodek, Olsen) & 8
\end{tabular}

\begin{tabular}{lll}
\hline 7 & NuTeV (Bodek, McFarland) & 9
\end{tabular}

8 MINERvA (Bodek, Manly, McFarland) 9

$\begin{array}{lll}9 & \text { T2K (Manly, McFarland) } & 10\end{array}$

$\begin{array}{ll}10 \text { JUPITER (Bodek, Manly) } & 10\end{array}$

11 Neutrino Interaction Phenomenology (Bodek, McFarland) 11

12 Light-by-Light Scattering at SLAC E-144 (Melissinos) 11

13 Axion Searches (Melissinos) 12

14 Novel Techniques in Accelerator Physics (Melissinos) 13

15 Theoretical Work of Ashok Das 13

16 Theoretical Work of C. Richard Hagen 13 


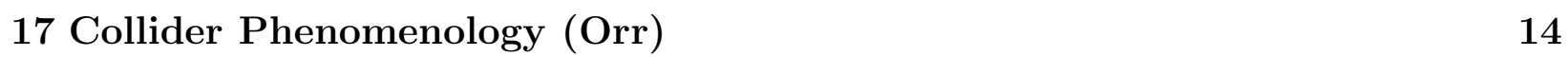

18 Theoretical Work of Susumu Okubo 15

$\begin{array}{ll}19 \text { Theoretical Work of Sarada Rajeev } & 15\end{array}$

$\begin{array}{lr}20 \text { Education and Public Outreach } & 16\end{array}$

21 Graduate Student and Postdoc Training $\quad 16$

$21.1 \mathrm{PhDs}$ in Experimental Particle Phvsics 1991-2012 . . . . . . . . . . . . . . 16

$21.2 \mathrm{PhDs}$ in Theoretical Particle Phvsics 1991-2012 . . . . . . . . . . . . 18

21.3 High Energy RAs/SRAs at Rochester 1991-2012 . . . . . . . . . . . . . . 19

22 Publications by Rochester Experiment group 1991 - $2012 \quad 21$

23 Publications by Rochester Theory group 1991-2012 201 


\section{CDF (Bodek, McFarland, Tipton)}

Bodek was in charge of the construction of the CDF plug-upgrade hadron calorimeters, Tipton was in charge of the construction and maintenance of the cooling system of the silicon vertex detector, and McFarland's group developed the Level-3 trigger, data logger and the event feed to the online monitoring for the experiment. The CDF plug upgrade calorimeter was the first large scale hadron calorimeter to use tile-fiber technology. Bodek and Haelen invented a new method to construct large multi-tile assemblies on an industrial scale (called megatiles). Bodek's group (Sakumoto) was in charge of the maintenance and calibration of the calorimeters, as well as CDF operations. McFarland served for a number of years as the leader of CDF offline software and data processing.

Tipton and Watts played a major role in the discovery of the top quark, and Tipton and Koehn measured the heavy flavor content of quark jets. Tipton and Tollefson performed the first measurement of the mass of the top quark. Tipton, Cassada and Coca measured the top pair production cross section and searched for new particles decaying to top-antitop pairs. Tipton and Ivanov searched for anomalous kinematics in dilepton events from top-antitop events. Tipton and Eusebi, and Bodek and G. B. Yu searched for the production of charged Higgs boson in top decays in different Higgs decay mode. McFarland and Demers measured the branching ratio of the top quark to tau leptons, and Bodek and Pillai searched for the production of Z' bosons using mass distribution of Drell-Yan events and published limits on quark-lepton compositeness, and Bodek and Baur published a paper on signatures of $Z^{\prime}$ boson in the forward-backward asymmetry of Drell-Yan pairs.

Bodek pioneered a series of measurements in hadron colliders that were used to provide information on parton distribution functions. Bodek and Dixon performed the first precise measurement of the $W$-lepton production asymmetry which was the first measurement of the ratio of down to up in hadron colliders. It was also the first hadron collider data to be incorporated into fits for Parton Distribution Functions (PDFs). Later Bodek and Fan published a new technique to measure the $W$ asymmetry in the forward direction beyond the tracking region of the central drift chamber. The technique involved extrapolating the tracks from the silicon vertex detector to the electromagnetic shower counter and using the position of the centroid of the EM shower to determine the charge of electrons and positrons. The technique was extended to the measurement of the production of $Z$ bosons as a function of rapidity to high rapidity (Bodek and J. Liu and later Bodek and J. Han). These measurements of the rapidity distribution of $Z$ boson were incorporated into PDF fits. In addition, Bodek and Sakumoto measured the transverse momentum distribution of $Z$ bosons.

Bodek, McFarland, and B.Y. Han published a new technique to unfold the $W$ decay lepton asymmetry to extract the $W$ production asymmetry. The results of the unfolded asymmetry are the only $W$ asymmetry data that are currently used in NNLO PDF fits (because these inclusive results do not require additional assumptions on modeling $W$ transverse momentum distributions). Bodek, Sakumoto and J. Han made the first measurement of angular distributions in the production of $Z$ bosons and determined the relative contribution 
of quark-antiquark annihilation and gluon-quark Compton process to $Z$ boson production.

Currently, Bodek and Sakumoto are working the analysis of the complete $10 \mathrm{fb}^{-1} \mathrm{CDF}$ Tevatron data sets in the single and dilepton channel. One of the goals is to extract a precise measurement of the electroweak mixing angle from the forward-backward asymmetry in Drell-Yan events. Bodek published a new event weighting technique to measure the asymmetry that improves the statistical precision of the measurement, and for which most of the systematic errors cancel. Bodek and Sakumoto also propose to repeat all of the above measurements with $W$ and $Z$ bosons with the full Tevatron sample. The large statistical samples will improve the precision of the measurements of $\mathrm{d} / \mathrm{u}$. In addition, a measurement of the ratio of the $W$ and $Z$ lepton rapidity distribution will result in an improved measurement of the strange sea.

\section{Do (Demina, Ferbel, Slattery)}

Rochester physicists played important roles in the Run II Upgrade of the D0 detector, including supplying the Visible Light Photon Counters (VLPCs) used to read out the Central Fiber Tracker (CFT) and central preshower detectors, co-leading the development of the Run II Online system, developing and testing the silicon sensors for the Layer 0 silicon tracker, and designing and installing the Analog Front End boards for the CFT readout. Rochester Senior Scientist, G. Ginther, served as D0's Technical Integration Coordinator from 2004-12.

At the beginning of Run II Demina and Khanov developed tracking and b-tagging algorithm for D0 data reconstruction. The main thrust of the Rochester analysis activities was the investigation of top quark. Demina, Khanov and Pleier measured top-antitop production cross section. SJ Park under Demina's supervision searched for an admixture of supersymmetric top quark production in top sample. In Run I Ferbel's group (Canelli and Estrada) developed an innovative matrix element algorithm to measured top quark properties including its mass. This approach was widely adopted in the field and extended to searches for new particles including Higgs. In 2005-2009 Demina led the D0 group on top quark mass measurements and supervised Rochester student C. Garcia in his work on the subject. Analysis of forward-backward asymmetry in top production performed by Demina, Harel and Orbaker yielded a 3 sigma discrepancy with the Standard Model prediction.

\section{CMS (Bodek, Demina, Slattery, Tipton)}

Bodek's group (Bodek, and senior scientists de Barbaro and Budd) was in charge of the construction of the tile-fiber optical detectors (megatiles) for the CMS hadron calorimeter (HCAL). The process for the construction of megatile was developed at Rochester when we built the plug hadron calorimeter for CDF. In parallel, the groups participated in numerous test beam runs that tested and mapped the response of a HCAL module in a hadron beam at CERN. Slattery and Ginther and Lobkowicz were in charge of the construction of the large and complex motion table to support and map the test beam HCAL module. Upon 
the completion of the construction of the optical elements, Dr. de Barbaro moved to CERN and was in charge of the installation of the megatiles in the CMS detector. After serving as deputy project manager of HCAL, de Barbaro was appointed as HCAL project manager.

Since 2000 Demina has served as a US project manager deputy, and for the period of 2010-2012 as a project manager, for the Silicon Tracker. Rochester was responsible for silicon sensor quality assurance (Korjenevski), shipping (Tipton) and long term testing of the rods (Gotra). The group played a key role in the detector commissioning at CERN working on Monte Carlo tuning (Gotra) and low level cluster reconstruction (Betchart).

Zielinski and Harel were responsible for jet algorithms and jet ID. As a result, Rochester played a key role in the search for contact interaction using dijet centrality ratio (thesis of D. Miner). After the start of the LHC run Demina and Flaetcher were responsible for the commissioning of missing energy for SUSY measurements. As a result, the first search for SUSY in the LHC was the one using the so-called $\alpha_{T}$ technique in an all-hadronic mode (Betchart, Flaetcher, Demina). Now Garcia-Bellido and Eshaq continue to apply this method to update SUSY searches with a larger statistics.

Bodek and Han have been working on precision electroweak physics and QCD studies using leptonic decays of $Z / W$ bosons and Drell-Yan events in the 2010, 2011 and 2012 data. They developed a new data technique to correct muon momenta for misalignment and scale. These corrections, which are called Rochester momentum corrections are necessary for precision SM studies. They remove all bias in the measurement of positive and negative muons as a function of $\eta$ and $\phi$, and reduce the contributions from muon momentum measurement to the error in the Higgs mass from $0.4 \%$ to $0.1 \%$. They also improve the mass resolution for $1 \mathrm{TeV}$ muons from $8 \%$ to $4 \%$. Bodek and Han have applied their experience on CDF to CMS measurements of $Z$ boson rapidity distribution and angular distribution, $W$ asymmetry, Drell-Yan mass and rapidity distributions, $W / Z$ ratios, and of the Drell-Yan forward-backward asymmetry, and search for $Z^{\prime}$ bosons and deviations from the standard model in the mass and angular distributions of dileptons. First results from these studies have already been published.

Using angular distributions in two Z-boson mode Covarelli and Demina performed a search for spin 2 Kaluza-klein graviton. The same technique is used for Higgs spin measurement. Demina, Covarelli, Garcia-Bellido and Goldenzweig are searching for VBF Higgs production in $\mathrm{ZZ}$ to two leptons, two quarks mode.

Betchart and Demina are measuring ttbar charge production asymmetry in CMS.

\section{$4 \quad$ E706 (Ferbel, Slattery)}

Fermilab experiment E706 was approved in 1981 as one of the initial round of Tevatron fixedtarget experiments. It was a second-generation study of neutral meson and direct-photon production by pions and protons on a variety of nuclear targets, including hydrogen. The E706 apparatus featured a 3-meter diameter liquid argon calorimeter to detect and measure photons and electrons and a large aperture multi-particle spectrometer to measure charged 
particles produced in association with direct-photons and neutral pions. P. Slattery served as Spokesperson for the experiment.

E706 had an engineering run in 1988, and two primary physics runs, the first in 1990 and the second in 1991-92. Results from E706 have had a significant impact on the development of perturbative QCD by demonstrating that conventional calculations of both neutral meson and direct-photon production, even at next-to-leading order (NLO), cannot reproduce the measured results. The discrepancy has been interpreted as being due to soft gluon processes not included in NLO calculations.

\section{ATLAS (Slattery)}

In 1992, following the last data run of E706, Slattery, together with NSF-funded physicist F. Lobkowicz, joined the GEM experiment at the SSC, where Rochester was assigned several major responsibilities, including supplying the hadronic modules for the proposed liquid krypton barrel calorimeter, and leading GEM's Fermilab-based test beam effort. After cancellation of the SSC, Slattery and Lobkowicz joined ATLAS at the CERN LHC, where Rochester provided the conceptual design for the ATLAS barrel cryostat and also completed a successful program of R\&D to develop an innovative high-density signal feed-through for all the ATLAS cryostats. In 1998, as part of a reorganization of Rochester's HEP activities following Lobkowicz's death, Slattery switched from ATLAS to CMS, where he helped design, construct and commission a large, computer controlled, motion table that has been extensively used in the CERN test beam program to study and calibrate elements of the CMS detector. He also joined D0 at that time.

\section{AMY (Bodek, Olsen)}

Rochester led the construction of the AMY $e^{+} e^{-}$experiment at the TRISTAN accelerator in Japan. Prof. Olsen served as the AMY spokesperson. Olsen led the construction of the AMY central drift chambers. Bodek led the construction of the muon chambers, gas system, muon scintillators and the online electronics. During this grant period several PhD theses on AMY were supervised by Bodek and Olsen. These include studies of the forwardbackward asymmetry in the production of dileptons (Bodek, C. Vellisaris), studies of photonphoton interactions (Bodek, B. J. Kim), extraction of $\alpha_{s}$ from events with jets (Bodek, Y. K. Lee), measurement of the hadronic cross section (Bodek, T. Kumita), measurements of multiplicities (Olsen, H. W. Zheng), studies of four lepton final states (Olsen, M. Y. Hong), and studies of the properties of quark versus gluon jets (Olsen, Young Kee Kim). 


\section{$7 \quad \mathrm{NuTeV}$ (Bodek, McFarland)}

The main goal of the $\mathrm{NuTeV}$ experiment was to measure charged and neutral cross-sections at high neutrino energies, from 20 to $400 \mathrm{GeV}$, in order to determine parton distributions and to test the predictions of the neutral to charged current ratio in the electroweak theory. The latter test is sensitive to possible unknown interactions beyond those of the standard model. Construction of the new beamline and refurbishing of the detector took place between 19941996 and the detector ran from 1996-1997. Rochester had responsibility for procurement and testing of photomultipliers, the design of the veto wall, the electroweak data anlalysis (McFarland) and the structure function analysis (Bodek, postdoc Harris, student Un-Ki Yang). Un-Ki Yang received the URA Fermilab best thesis award for his work on structure functions. Bodek and deBarbaro searched for neutral heavy leptons, and Bodek and student Avvakumov completed a search for high $\delta m^{2} \nu_{\mu} \rightarrow \nu_{e}$ oscillations. The electroweak analysis resulted in a paper with over 500 citations, and a Tanaka dissertation award for student Sam Zeller who collaborated extensively with McFarland on the work.

\section{MINERvA (Bodek, Manly, McFarland)}

MINERvA is an experiment designed to study interactions of neutrinos and anti-neutrinos with energies between 1.5 and $15 \mathrm{GeV}$. Improved knowledge of these interactions is a critical input for current and future neutrino oscillation experiments. The data also provides a laboratory for strong interaction processes which cannot be calculated from first principles in our current frameworks, but which can be modeled by various effective theories which give insight into the phenomenology of these complex strongly bound systems.

The University of Rochester group wrote the original proposal for MINERvA in 2002 which included the basic detector design. Prof. McFarland has served as co-spokesperson since the formation of the collaboration. The University of Rochester group played many key roles in the construction of the experiment, including prototyping of the detector, construction of the optical fiber systems, development of the data acquisition system and final assembly of the modules and the detector. The detector construction project won the Secretary of Energy's Award of Achievement in 2011. After detector construction finished in 2010, postdoc Gabriel Perdue and then senior scientist Howard Budd have served as run coordinators during the operations phase.

The University of Rochester group also plays key roles in the data analyses completed to date. Graduate student Jesse Chvojka's thesis on the process $\bar{\nu}_{\mu} p \rightarrow \mu^{+} n$ was the first completed cross-section measurement from the experiment in 2011. Postdoc Gabriel Perdue is currently the deputy analysis coordinator for the experiment, and Prof. Manly leads the electromagnetic final states analysis group. Prof. Bodek is leading the flux extraction using an improved low $\nu$ method. 


\section{$9 \quad$ T2K (Manly, McFarland)}

Our group began an involvement in $\mathrm{T} 2 \mathrm{~K}$ as the concept for the experiment was being formed in 2001. Our initial efforts focused on development of the near detector, ND280, and Prof. McFarland was one of the initial co-conveners of the first T2K near detector working group. Rochester played important roles in designing and constructing parts of the near detector, particularly the $\pi^{0}$ detector (P0D). Most of the electronics for the near detector is based on the design of the MINERvA electronics, the fiber readout and mirroring scheme was prototyped and developed by Rochester originally in the context of MIENRvA and P0D uses scintillator extrusions developed for MINERvA. In this way, we were able to realize synergies between our MINERvA and T2K construction efforts which ultimately led to significant savings and reduced techincal risk for the US T2K project whose early construction lagged MINERvA by a year. Melanie Day made the first measurement of the electron neutrino content of the T2K beam as her thesis, and this was an important supporting measurement for the initial indications that the rate $\nu_{\mu} \rightarrow \nu_{e}$ was large from T2K in 2011.

Much of our current effort focuses on modeling of neutrino reaction cross-sections for T2K's oscillation analyses. Prof. McFarland co-convenes the T2K group responsible for the model and for fitting data to the model (Neutrino Interactions Working Group, or NIWG), and most of the active Rochester personnel on $\mathrm{T} 2 \mathrm{~K}$ work in this area which has obvious synergies with the work on MINERvA. Prof. Manly's measurements of pion electroproduction on different nuclear targets, described below, are one of the important constraints that go into this cross-section model.

\section{JUPITER (Bodek, Manly)}

The purpose of the JUPITER program (Bodek and Manly) is to use electron scattering data measured at Jefferson lab to study vector current processes of interest for one-few $\mathrm{GeV}$ neutrino scattering. This data can be used to build models of neutrino cross-sections and provide a theoretical framework for the interpretation of MINER $\nu \mathrm{A}$ data.

Bodek is the co-spokesperson of the Hall $\mathrm{C}$ component of the JUPITER program (JLab experiments E04-001, JLab E02-109). These two electron scattering experiments measure separated vector structure functions $, F_{2 p}, F_{2 n}, R_{p}, R_{n}$ for free and bound nucleons in the quasielastic, resonance and inelastic regions. The data is used to determine the vector component of the structure function in neutrino scattering experiments. These new data taken on nuclear targets are expected to reduce the errors on the vector cross sections by a factor of 10 .

Manly is leading the Hall B component of the JUPITER program which makes use of the full acceptance CLAS experiment data. Hyupwoo Lee, working with Prof. Manly, is producing differential pion production cross sections on different nuclear targets for his Ph.D. thesis project. These results will be useful for tuning models of neutrino resonance production as well providing constraints on backgrounds and nuclear final state interactions in neutrino experiments 


\section{Neutrino Interaction Phenomenology (Bodek, Mc- Farland)}

Bodek's work in electron scattering and neutrino interaction phenomenology provide models for neutrino interactions Monte Carlo (such as GENIE). These include the Bodek-Ritchie model for Fermi motion, and the Bodek-Yang model for structure functions in the inelastic region.

Bodek was the spokesperson of SLAC experiment E140, which yielded very precise measurement of separated structure functions, $F_{2}$ and $R$. Bodek's parametrization of $R$ is used in the Bodek-Yang model. The precise measurements of SLAC E140, were used to do a combined analysis of all electron scattering data at SLAC, which were cross normalized to SLAC E140 (Bodek and Rock). The BCDMS muon data was then cross normalized to the SLAC data to provide structure data over a very wide range of $Q^{2}$.

Bodek and Yang's analysis of electron scattering data determined that the higher twist corrections that are needed to describe the SLAC data within a NLO model were mostly due to the missing QCD NNLO terms. The Bodek-Yang model that describes all of the structure functions from $Q^{2}=0$ to very high $Q^{2}=0$ uses a new scaling variable to account for target mass and the missing higher order terms, in combination with $K$ factors at very low $Q^{2}$.

Recently, Bodek and Budd used the JUPITER data for quasielastic electron scattering on nuclear targets to extract a parametrization of the Transverse Enhancement which is observed in electron scattering. This enhancement is expected from meson exchange currents. If this transverse enhancement is included in the theoretical predictions for quasielastic scattering in neutrino reactions, it resolves the long standing discrepancy between MiniBooNE data and higher energy neutrino experiments, thus resolving what has been called "the axial mass anomaly".

\section{Light-by-Light Scattering at SLAC E-144 (Melissi- nos)}

In the early 1990s we carried out an experiment at SLAC to examine non-linearities in electromagnetic scattering. The experiment was a collaboration between Rochester-PrincetonSLAC and the Un. of Tennessee. A high intensity laser beam was back-scattered from the $\sim 50 \mathrm{GeV}$ electrons in the Final Focus Test Beam. The intensity at the focus (diameter $\sim 10 \mu \mathrm{m}$ ) of the laser pulse (energy $\sim 1 \mathrm{~J}$ ) and of duration 1-2 ps was of order $I=10^{18} \mathrm{~W} / \mathrm{cm}^{2}$. This corresponds to an electric field at the focus $E_{L} \sim 2 \times 10^{10} \mathrm{~V} / \mathrm{cm}$. However in its rest frame the electron $\left(\gamma \sim 10^{5}\right)$ sees an electric field $E^{*}=2 \gamma E_{L} \sim 4 \times 10^{15}$, which is of order of $1 / 3$ of the critical field strength, $E_{c}=\left(m^{2} c^{3}\right) /(e \hbar)=1.3 \times 10^{16} \mathrm{~V} / \mathrm{cm}$. At such field strength we expect the vacuum to break down spontaneously by the production of $e^{+} e^{-}$pairs, as first shown by Schwingen 1 . Note the importance of the highly relativistic

\footnotetext{
${ }^{1}$ Phys. Rev., 82, 664 (1951)
} 
electron beam in increasing the effective laser intensity by a factor of $10^{10}$, which can not be achieved in the laboratory, even in future high power lasers.

At these high electric fields, non-linear effects are dominant, and drive multi-photon processes. We studied Compton scattering involving the absorption of up to 5 photons from the laser field. On occasion the backscattered high energy photon interacted again within the laser focus, with at least 4 photons, to produce an $e^{+} e^{-}$pair. A precise description of the observed process is "photon-multiphoton inelastic scattering", and the rate agrees exactly with the perturbative calculation, but also with the non-perturbative calculation of Schwinger; this because the electric field is near the critical value, but neither well below nor much higher than $E_{c}$. This is the familiar case where the same physical process can be interpreted in two different frameworks.

Our SLAC experiment was the first time that critical field was reached in the laboratory and that the "breakdown of the vacuum" was demonstrated. It was also the first time that light-by-light scattering in the absence of matter (in the vacuum) was observed in a laboratory experiment. Finally, it also provided evidence for the treatment of the non-linear aspects of the electromagnetic field at energies much higher than in the conventional quantum optics experiments.

\section{Axion Searches (Melissinos)}

Our group was the first to carry out a search for cosmic axions by their conversion into microwave photons in a cavity placed in a magnetic field, as proposed by P.Sikivie. The experiment was carried out at Brookhaven National Laboratory in the late 1980s, and covered the range of axion masses $4 \leq m_{a} \leq 20 \mu \mathrm{eV}$ without observing a signal, but not with sufficient sensitivity to exclude the theoretical predictions on the strength of the axion coupling to two photons, given the expected local axion density. While considerable effort has been invested since then to improve these limits (the ADMX project) the Rochester-Brookhaven-Fermilab results represent, even today, the widest coverage in axion mass in the $\mu \mathrm{eV}$ range.

In the early 1990s we initiated a new experiment to create axions using optical techniques. In this case the polarization vector of the light traversing a magnetic field is rotated if axions with $m_{a}<\hbar \omega$ are produced, or becomes elliptically polarized when $m_{a}>\hbar \omega$. In spite of the negative results, such experiments are still being continued at other laboratories, even at Fermilab, but have not improved on the limits set by the series of the RochesterBrookhaven-Fermilab-Trieste experiments. In general the sensitivity that can be reached in optical experiments is much less than that obtained from the search for cosmic axions, but the optical experiments have the advantage that they do not demand the presence of an axion background and they cover a broad mass range in a single measurement. Finally, we mention the first search for axions produced in the sun, that would have typical energies in the X-ray region, carried out by our group at Brookhaven. 


\section{Novel Techniques in Accelerator Physics (Melissi- nos)}

Our group participated in the establishment and operation in the mid 1990s of the "A0 Test Facility" at Fermilab. The facility was based on a prototype low emittance electron accelerator. The accelerator consisted of an rf photoinjector followed by a 9-cell Niobium suoerconducting cavity operating at $1.3 \mathrm{GHz}$. The final electron energy was $\sim 18 \mathrm{MeV}$. The photoinjector was driven by short UV pulses of duration few to $10 \mathrm{ps}$, in a train of 1-1000 pulses spaced $1 \mu \mathrm{s}$ apart, so as to match the specifications of the TESLA design for an $e^{+} e^{-}$ collider. The cathode was $\mathrm{Cs}_{2} \mathrm{Te}$ and the charge yield $\sim 10 \mathrm{nC}$, or $6 \times 10^{10}$ electrons per pulse. Rochester built, installed and operated the drive laser, and participated in many of the experiments carried out at the A0 facility.

Most notable among the experiments was the first demonstration of electro-optic sampling of a high energy electron beam (M.Fitch), now an important diagnostic for short pulse intense electron beams. Other experiments involved the production of "flat beams" and several diagnostics, including the use of transition radiation. We also participated in the completion and commissioning of the electron cooling of the antiproton beam in the Fermilab accumulator (S.Seletskiy).

Rochester's involvement, and the contribution of our graduate students, were a key factor in the establishment of the A0 test facility, and its operation over the next decade.

\section{Theoretical Work of Ashok Das}

During the grant period his research has focussed around many areas in theoretical physics including quantum field theory, field theory at finite temperature, integrable models, string theory and even some works on phenomenology. The works were done in collaboration with colleagues from various countries as well as with students and post doctoral fellows here at the University of Rochester. During this period, his research has led to over 200 published papers, about 30 conference reports, 13 published books and several hundred invited talks at various institutions. This has also led to several $\mathrm{Ph}$. D. theses of graduate students.

\section{Theoretical Work of C. Richard Hagen}

During the time period covered by this report, Hagen has pursued topics in such diverse areas as Aharonov-Bohm and Aharonov-Casher effects, Galilean relativity, two dimensional field theories and the Casimir effect. Particularly important in the first category are his results presenting the first correct treatment of the spin- $1 / 2$ Aharonov-Bohm effect and the demonstration of equivalence between the Aharonov-Casher effect and the spin-1/2 Aharonov-Bohm effect. These results set the standard for ongoing research in this field. He has used Galilean relativity to analyze some formal issues as well as to provide a framework for Aharonov-Bohm calculations in which a coulomb potential is also considered. His work on two dimensional 
field theory culminated in formulating and solving the most general soluble field theory which yields in various limits ALL the previously known soluble cases (Thirring model and Schwinger model to name just two). His work on the Casimir effect served to resolve some technical issues which existed in many of the early calculations in this subfield. Finally, his work with Nicholas Bigelow resolved issues surrounding a claim of superluminal wave propagation.

\section{Collider Phenomenology (Orr)}

The Collider Phenomenology group, led by Professor Lynne H. Orr, has covered a number of topics within and beyond the Standard Model (SM) at the Fermilab Tevatron, CERN LHC, and a future high energy electron-positron collider. In top quark physics, our primary focus, we have studied the effects of Quantum Chromodynamics (QCD), including the effects of gluon radiation on measuring top properties as well as computing higher order QCD corrections to top production and decay processes. We have also investigated the measurability of anomalous couplings to top quarks of photons and $Z$ bosons at the Tevatron and LHC. In other work, we have considered so-called boosted tops, which are produced at high energies and can be difficult to identify. We found methods to significantly improve the chances of discovering new heavy particles in the $t \bar{t}$ channel at the LHC.

We have also computed full next-to-leading-order QCD corrections to production of $t \bar{t}$ in association with a Higgs boson at the Tevatron and LHC. This production mode will be very important for determining the strength of the Higgs coupling to the top quark, and in particular whether this coupling deviates from its expected SM value. Also in electroweak theory, our group contributed to the next-to-leading order electroweak calculation of $W^{+} W^{-}$ production at LEP, and the incorporation of this calculation in the publicly available computer program RACOONWW. In pure QCD we have improved on the standard calculation of the solution of the Balitsky-Fadin-Kuraev-Lipatov (BFKL) equation for summation of soft gluons in forward jet production, by constructing a Monte Carlo generator that incorporates kinematic constraints on the process.

Our work in collider phenomenology beyond the SM has contributed to several kinds of physics models. In Supersymmetry (SUSY), we have identified a simple parameterization for SUSY models which do not incorporate the discrete symmetry R-parity, and explored the resulting phenomenology. We have contributed to the SUSY Madgraph program. We have also investigated the breakdown of the narrow width approximation in production and decay of SUSY particles. This common approximation is widely used in studies, but is generally invalid for the heavy particles produced in SUSY. In Little Higgs models, we have studied the phenomenology of a new heavy neutral gauge bosons, including placing new limits from direct searches and calculating the tree-level decay width and branching ratios the Littlest Higgs. We have also studied the production via SM gauge-boson fusion and decay of gauge bosons in the Simplest Higgs model. Finally, in deconstructed Higgsless models we have also studied production of new heavy gauge bosons via gauge-boson fusion. 


\section{Theoretical Work of Susumu Okubo}

Okubo's collaborations with Professors Kamiya and Elduque of Japan and Spain, respectively were initially concerned with Jordan algebras. Jordan algebras are intimately related to the theory of observables in quantum mechanics. He has constructed exceptional Lie superalgebras $D(2,1 ; \alpha), G(3)$, and $F(4)$ from triple systems related to quarternion and octonions. Okubo recently has been concentrating on mathematical physics with an emphasis on applications of non-associative algebras to physics. It has been known for some time that the octonion algebra satisfies the so called triality relation. More recently, he has shown that many algebras including the Lie algebra $E_{8}$ do possess similar properties together with $S_{4}$-symmetry. He is pursuing the problem in more depth with A. Elduque of the University of Zaragoza in Spain, N. Kamiya of Aizu University in Japan and D. Mondac of Lund University in Sweden.

\section{Theoretical Work of Sarada Rajeev}

Rajeev's seminal work on the Skyrme model led him to a study of other quantum field theories, with an emphasis on gauge theories and an occasional foray into string theory. He has developed mainly geometrical methods that build on the large $\mathrm{N}$ limit and path integrals that apply as well to other areas of physics. The exact solution of two dimensional pure Yang-Mills theory and the determination of the quantum theory of hadrons (including baryon masses) in two dimensions were among the early results. Renormalization beyond perturbation theory was developed in several models of quantum mechanics and later, quantum field theory. Rigorous construction of renormalized path integrals were given to show that it is possible to make mathematical sense of divergent quantum theories. Soliton models of parton structure functions was an application of phenomenological interest. This work has led to a Finite Element Approach to quantum field theory based on Discrete Differential Geometry, which is a current research interest. This work has been published in over a hundred papers and $10 \mathrm{Ph}$. D. theses. 


\section{Education and Public Outreach}

Launched in 1994, the Pre-College Experience in Physics (PREP) program is the only extensive program in science for high school girls in the Rochester area. PREP received initial funding from the U. S. Department of Energy's (now-terminated) Office of Science Education (1994-96), and the US-CMS collaboration has provided partial funding for the continuation of the program (since 1998). The all-day, 4-week summer program serves 24 high school students, and employs at least two women undergraduate or graduate students as instructors. Now entering its eighteenth year, PREP-CMS has involved about 375 young women in the Rochester community and 34 graduate and undergraduate instructors. Manly is the program director.

Bodek is the PI for the NSF Research Experience for Undergraduate and Research Experience for Teachers (REU/RET ) program at Rochester. The NSF grant provides partial support for undergraduates to do particle physics research. Undergraduates who do not meet NSF eligibility requirements (US residents) are supported by the DOE grant. One of our REU undergraduates won the APS Apker award for best undergraduate thesis.

McFarland is the PI for Rochester QuarkNet program for high school teachers, who were trained to take data with cosmic ray telescopes and bring these detector to their high school. The PARTICLE (Physicists and Rochester Teachers Inventing CLassroom Experiments) program at the University of Rochester now is entering its tenth year. PARTICLE has trained 36 area teachers from 28 different schools in the use of cosmic ray detector for classroom research use. It maintains a lending library of these detectors, and provides support for participating classrooms. PARTICLE also maintains a $3 \mathrm{~m}^{2}$ continuously operating surface cosmic ray detector which has been taking data since 2005 and is used for high-school student research into long-term studies of cosmic ray rate variations with solar activity. In the recent past, PARTICLE teachers and students refurbished $\mathrm{NuTeV}$ scintillator panels into the hardware for MINERvA's veto wall. In summer 2010 and 2011, the program supported a team of nine teachers to develop lesson plans to use data streams from MINERvA of quasielastic scattering candidates and stopping muons to study elastic scattering kinematics and radioactive decay.

\section{Graduate Student and Postdoc Training}

\subsection{PhDs in Experimental Particle Physics 1991-2012}

\begin{tabular}{lcll} 
Name & Ph.D Year & Thesis Expt. & Current Position/Expt. \\
\hline R. Cameron & 1992 & Axions E840 & Industry \\
C. Bamber & 1992 & Laser-Linac & National Research Council, Canada \\
T. Kumita & 1992 & AMY & RA INS, U. of Tokyo \\
Y.K. Li & 1993 & AMY & Industry, Hughes Aerospace \\
S. Durston-Johnson & 1993 & DZero & Sr. Lecturer Simon Frazer Univ. \\
N. Varelas & 1994 & FNAL-E706 & Prof., U. of Illinois at Chicago
\end{tabular}


R. Roser

M. Dickson

J. Borders

R. Hirosky

G. Watts

L. de Barbaro

B.J. Kim

C. Velissaris

C. Cretsinger

T. Kotseroglou

S. Boege

M. Pillai

G. Osborne

A. Fry

K. Fatyga (NSF)

P. Koehn

E. Won

K. Tollefson

D. Casey

T. Koffas

K.S. Hahn

M. Begel

D. Reis

J. Cassada

M. Fitch ${ }^{1}$

J.B. Liu

U.K. Yang ${ }^{2}$

S. Avvakumov

J. Estrada ${ }^{3}$

M. Florencia Canelli ${ }^{4}$

Benjamin Kilminster ${ }^{5}$

Gregory Davis

William Butler

Sarah Demers Konezny

Andrew Ivanov

Mircea Coca

Alexander Khanov

Doo Kee Cho

Sergei Seletskiy

Kwok ming (Leo) Chan

Rodian Tikhoplav

Phil Yoon

SuJung Park

Carlos Garcia

Jiyeon Han
1994

1994

1994

1994

1994

1995

1995

1995

1996

1996

1996

1996

1996

1996

1996

1996

1996

1997

1997

1998

1998

1999

1999

1999

2000

2000

2001

2001

2001

2003

2003

2004

2004

2004

2004

2004

2004

2005

2005

2006

2006

2007

2007

2007

2008
FNAL-E706

CDF

DZero

DZero

CDF

FNAL-E706

AMY

AMY

DZero

SLAC/QED

SLAC/QED

$\mathrm{CDF}$

E706

FNAL/A $\emptyset$

DZero

CDF

DZero

CDF

DZero

SLAC/QED

DZero

FNAL-E706

SLAC/QED

$\mathrm{CDF}$

FNAL/A $\emptyset$

CDF

CCFR/NuTev

$\mathrm{NuTeV}$

DZero

DZero

CDF

DZero

LIGO

CDF

CDF

CDF

DZero

DZero

FNAL/A0

DZero

FNAL/A0

FNAL/Accel

DZero

Dzero

CDF
Scientist, Fermilab

Scientist, MIT Lincoln Labs

Analyst, Credit Suisse, N.Y.

Assoc. Prof., U of Virginia

Prof., U. of Washington

Scientist, Lucent Labs

Asst. Scientist, University of Florida

Lecturer, U. Central Florida

Patent Attorney, San Francisco

Industry

Scientist, Opt. Sys, Life Technologies

Industry, India

Industry, Rochester

Scientist, SLAC

retired

Research Staff, Inst. for Defense Analyses

Asst. Prof., Korea University

Assoc. Prof., Michigan State University

Faculty, St. John's College, Maryland

Asst. Prof., Carleton University

Lucent Industries, IL

Scientist, Brookhaven Nat'l Lab.

Assoc. Professor, Stanford University

U.S. Naval Aviator

Researcher, Johns Hopkins Applied Physics Lab

Completing MBA, U. of Toronto

Lecturer, University of Manchester

Industry, Finance

Scientist, Fermilab

Prof. Univ. of Zurich

Prof. Univ. of Zurich

Research, Inst. for Defense Analyses

Sr. Processing Eng., Intel, Portland, OR

Asst. Prof., Yale U.

Asst. Prof., Kansas State U.

UR medical school

Asst. Prof. Oklahoma State

RA, Brown University (DZero)

Scientist, Brookhaven Nat'l Lab, SSRL

RA, University of Notre Dame

Industry

Industry

Industry

Industry, Finance

RA, University of Rochester (CMS) 


$\begin{array}{llll}\text { Stefanos Giampanis } & 2008 & \text { LIGO } & \text { RA, University of Wisconsin, Milwaukee } \\ \text { BoYoung Han } & 2008 & \text { CDF } & \text { Korea Atomic Energy Research Inst. } \\ \text { Jennifer Gimmell } & 2009 & \text { CDF } & \text { Asst. Prof., College of DuPage } \\ \text { Geumbong Yu } & 2009 & \text { CDF } & \text { RA Duke University (CDF/ATLAS) } \\ \text { Douglas Orbaker } & 2011 & \text { D0 } & \text { RA University of Rochester }\end{array}$

${ }^{1}$ Awarded most outstanding thesis on research conducted at Fermilab by the URA, 2001.

2 Awarded most outstanding thesis on research conducted at Fermilab by the URA, 2002 and Lobkowicz Prize 2002.

${ }^{3}$ Awarded Tollestrup Award for Postdoctoral Research done at Fermilab by the URA, 2003 (based partially on his thesis) and Lobkowicz Prize 2001.

4 Awarded URA Best Thesis Award 2004, Lobkowicz Prize 2004 and APS Tanaka Prize 2006.

5 Awarded the Lobkowicz Prize 2004.

\subsection{PhDs in Theoretical Particle Physics 1991-2012}

\begin{tabular}{lcll} 
Name & Ph.D Year & Thesis Expt. & Current Position/Expt. \\
\hline G. Ferretti & 1993 & S. Rajeev & Prof., Chalmers University,Sweden \\
P. Bedaque & 1994 & A. Das & Prof., University of Maryland \\
I. Horvath & 1994 & A. Das & Research Prof., University of Kentucky \\
S. Guruswamy & 1995 & S. Rajeev & Lecturer at UC Santa Barbara \\
P. Vitale & 1995 & S. Rajeev & Asst. Prof., University of Naples, Italy \\
O. Turgut & 1996 & S. Rajeev & Director, Gursey Institute, Turkey \\
R. Henderson & 1997 & S. Rajeev & J.P. Morgan Bank, NYC \\
C.-W. Herbert Lee & 1999 & S. Rajeev & Bank of China, Hong Kong \\
Cosmin Macesanu $^{2}$ & 2001 & L. Orr & Wave Imaging Technology, Texas \\
Silvana Perez $^{3}$ & 2003 & A. Das & Professor, Univ. Federal do Para, Brazil \\
Levent Akant $^{4}$ & 2003 & S. Rajeev & Teaching Position, Bogazici Univ., Turkey \\
Alexander Mitov & 2003 & L. Orr & Theory Fellow, CERN \\
Govind Krishnaswami & 2004 & S. Rajeev & Asst. Prof., Chennai Math Inst., India \\
Alin Constandache & 2004 & A. Das & Publishing, Boston \\
Arsen Melikyan & 2006 & A. Das & Prof., University of Brasilia, Brazil \\
A. Agarwal & 2006 & S. Rajeev & Editor at Physical Review Letters \\
John Boersma & 2007 & L. Orr & Adjunct Prof. UR and Educational Software \\
Dan Berdine & 2010 & L. Orr & Defense Intelligence Agency, Washington, DC
\end{tabular}

${ }^{1}$ Worked at Rochester, final degree from University of Naples.

2 Awarded Okubo Prize 1997.

3 Worked at Rochester, final degree from Universidade de São Paulo.

4 Awarded Okubo Prize 1999.

5 APS Apker Award 2000 and Okubo Prize 2001. 


\subsection{High Energy RAs/SRAs at Rochester 1991-2012}

\begin{tabular}{|c|c|c|c|}
\hline Name & Position & $\begin{array}{l}\text { At UR } \\
\text { (SRA) }\end{array}$ & Current Position \\
\hline Vishnu Mathur & SRA/Faculty (theory) & $1968-1996$ & Prof. Emerit., Rochester \\
\hline Howard Budd & RA (CCFR) & 1983(1989)-present & SRA, Rochester (MINERvA) \\
\hline Koji Ueno & SRA (AMY) & $1984-1992$ & SRA, Natl Taiwan Univ/KEK \\
\hline Willis Sakumoto & 1984-present & Scientist, Rochester (CDF) & \\
\hline Gerald Blazey & RA (D0) & 1986(1990)-1994 & Professor, Northern Illinois U. \\
\hline Paul Draper & $\mathrm{RA}(\mathrm{D} 0)$ & 1986(1990)-1992 & Faculty, U Texas $\rightarrow$ science publishing \\
\hline Joe Rogers & RA (Axions) & $1987-1991$ & Professor, Cornell (deceased 2004) \\
\hline Alan Sill & RA (AMY/CDF) & 1987(1990)-1992 & Sr. Scientist, U. Texas, Lubbock \\
\hline Marek Zielinski & SRA (E706/DØ) & 1987-present & Senior Scientist, Rochester (DØ/CMS) \\
\hline James Dunlea & RA (ATLAS) & 1988(1993)-1994 & Industry \\
\hline Priscilla Auchincloss & $\mathrm{RA}(\mathrm{CCFR})$ & 1989-1997 & Dean of Students UR $\rightarrow$ Education \\
\hline Giovanni Cantatore & RA (Axions) & 1989-1990 & Scientist, INFN Trieste \\
\hline Robert Walker & $\mathrm{RA}(\mathrm{CDF})$ & 1989-1993 & Industry \\
\hline Leigh Chinitz & RA (AMY) & $1990-1993$ & Industry, Scientist, Motorola \\
\hline Pawel de Barbaro & RA (CCFR) & 1990(1997)-present & Senior Scientist, Rochester (CDF/CMS) \\
\hline Jan Ftacnik & RA (NSF E706) & $1990-1994$ & CERN \\
\hline Sunichi Kanda & RA (AMY) & 1990-1992 & KEK \\
\hline Yigao Liang & RA (Theory) & 1990-1992 & Industry \\
\hline John Mansour & RA (E706) & 1990-1992 & Industry \\
\hline Yannis Semertzidis & RA (Axions) & 1990-1992 & Scientist, BNL \\
\hline Hongwei Zheng & $\mathrm{RA}(\mathrm{CDF})$ & 1990-1991 & $\mathrm{SSC} \rightarrow$ Industry \\
\hline J. Barcelos-Neto & RA (Theory) & 1991-1992 & Prof. Emerit., U. Rio deJaneiro \\
\hline William Desoi & $\mathrm{RA}(\mathrm{E} 706)$ & 1991-1993 & Industry \\
\hline Giuseppe Ruoso & RA (Axions) & 1991-1992 & Scientist INFN, Italy \\
\hline Brian Winer & $\mathrm{RA}(\mathrm{CDF})$ & 1991-1996 & Assoc. Prof., Ohio State U. \\
\hline Zhu Yang & RA (Theory) & 1991-1993 & Industry \\
\hline Charles Bamber & RA (SLAC E144) & $1992-1995$ & INat'l Research Council, Canada \\
\hline Stefan Gruenendahl & RA (D0) & 1992(1996)-1998 & Scientist, FNAL \\
\hline Kumar Gupta & RA (Theory) & 1993-1995 & Professor, Saha Inst., India \\
\hline Richard Hughes & $\mathrm{RA}(\mathrm{CDF})$ & 1993-1996 & Assoc. Prof., Ohio State \\
\hline Vladimir Glebov & SRA (ATLAS) & 1994-1996 & Scientist, Rochester Laser Laboratory \\
\hline Yi Cen & RA (ATLAS) & 1994-1995 & Industry \\
\hline Deborah Harris & $\mathrm{RA}(\mathrm{NuTeV})$ & 1994-1999 & Scientist, FNAL \\
\hline Chung Kao & RA (theory) & 1994-1996 & Assoc. Prof., U. of Oklahoma \\
\hline Bockjoo Kim & RA (CDF-Plug) & 1994-1997 & Scientist, U. of Florida (iVDGL) \\
\hline Marc Paterno & RA (D0) & 1994(1998)-1998 & Scientist, FNAL \\
\hline Jaehoon Yu & RA (D0) & 1994-1996 & Assoc. Prof.,U. Texas-Arlington \\
\hline Kamel Bazizi & RA (ATLAS) & 1995(1996)-1997 & Industry \\
\hline Sergio Pernice & RA (Theory) & $1995-1997$ & Professor, U. Buenos Aires \\
\hline Theo Kotseroglou & RA (SLAC E140) & $1995-1996$ & Staff, SLAC \\
\hline Alan Fry & RA (SLAC E144) & 1996-1996 & Scientist, SLAC \\
\hline Mark Kruse & $\mathrm{RA}(\mathrm{CDF})$ & 1996-1999 & Assoc. Prof., Duke \\
\hline Steven Blusk & $\mathrm{RA}(\mathrm{CDF})$ & $1996-2001$ & Assoc. Prof., Syracuse \\
\hline Michael Bisset & RA (Theory) & $1997-1998$ & Professor, Tsinghua University \\
\hline Otto Kong & RA (Theory) & 1997-1999 & Professor, National Central U., Taiwan \\
\hline Thomas Koffas & RA (SLAC E144) & 1998-1999 & Asst. Prof. Carleton U. \\
\hline
\end{tabular}




$\begin{array}{lcc}\text { Robert Szalapski } & \text { RA (Theory) } & 1998-1999 \\ \text { Volker Buescher } & \text { RA (D0) } & 1998-2000 \\ \text { Kirsten Tollefson } & \text { RA (CDF) } & 1998-2002 \\ \text { Inkyu Park } & \text { RA (PHOBOS) } & 1998-2004 \\ \text { Varghese John } & \text { RA (Theory) } & 1999-2000 \\ \text { Michael Fitch } & \text { RA (A0) } & 1999-2000 \\ \text { Gennaro Corcella } & \text { RA (Theory) } & 1999-2001 \\ \text { Doreen Wackeroth } & \text { RA (Theory) } & 1999-2002 \\ \text { Tony Vaiciulis } & \text { RA (CDF) } & 1999-2005 \\ \text { Michael Begel } & \text { RA (Dzero) } & 1999-2007 \\ \text { Yonsei Chung } & \text { RA (CDF/CMS) } & 1999-2010 \\ \text { Andy Hocker } & \text { RA (CDF) } & 2000-2005 \\ \text { Wojtek Skulski } & \text { RA (NSF PHOBOS) } & 2000-2004 \\ \text { Eva Halkiadakis } & \text { RA (CDF) } & 2001-2005 \\ \text { Adrian Ghinculov } & \text { RA (Theory) } & 2002-2003 \\ \text { Gilles DeLentdecker } & \text { RA (CDF) } & 2003-2005 \\ \text { Jianliang Li } & \text { RA (A0) } & 2003-2006 \\ \text { Veronique Boisvert } & \text { RA (CDF) } & 2004-2007 \\ \text { Yuri Gotra } & \text { RA (DØ/CMS) } & 2004-2010 \\ \text { David Rainwater } & \text { RA (phenom) } & 2004-2008 \\ \text { Arnulf Quadt } & \text { RA (DØ) } & 2004-2006 \\ \text { Jochin Cammin } & \text { RA (DØ) } & 2004-2009 \\ \text { Robert Bradford } & \text { RA (MINERvA) } & 2005-2010 \\ \text { Ulrich Husemann } & \text { RA (CDF) } & 2005-2006 \\ \text { Sergey Avvakumov } & \text { RA (MINERvA) } & 2006-2008 \\ \text { Amnon Harel } & \text { RA (CMS) } & 2006-\text { present } \\ \text { Matsuo Sato } & \text { RA (Theory) } & 2006-2007 \\ \text { John Boersma } & \text { RA (Theory) } & 2008-2009 \\ \text { Christian Stahn } & \text { RA (Theory) } & 2008-2009 \\ \text { Gianluca Petrillo } & \text { RA (Dzero) } & 2008-\text { present } \\ \text { Gabriel Purdue } & \text { RA (MINERvA) } & 2008-\text { present } \\ \text { Jiyeon Han } & \text { RA (CMS) } & 2008-\text { present } \\ \text { Henning Flaecher } & \text { RA (CMS) } & 2010-\text { present } \\ \text { Pablo Goldenzweig } & \text { RA (CMS) } & 2010-\text { present } \\ \text { Roberto Covarelli } & \text { RA (CMS) } & 2011-\text { present } \\ \text { Philip Rodrigues } & \text { RA (T2K) } & \\ \text { Laura Loiacono } & \text { RA (MINERvA) } & \\ & & \end{array}$

Adjunct Faculty, SUNY Brockport

RA, Gutenberg U., Germany

Assoc. Prof., Michigan State

Assist. Prof., KAIST Inst., Korea

Industry

Researcher, Johns Hopkins, Applied Physics

RA, MPI-Munich

Assoc. Prof., Buffalo

Industry

Physicist, BNL

Research Fellow, Korea Rare Isotope Accelerator

Associate Scientist, Fermilab

Sr. Scientist, UR LLE

Asst. Prof., Rutgers University

Industry

SRA, U. Libre, Brussels

Industry

Lecturer, Royal Holloway Univ.

Industry

Scientist, U. Texas, Applied Research Lab

Faculty, Inst. Phys. Gottingen

RA, Johns Hopkins, Medical Imaging

Scientist, Argonne

Scientist, DESY

Industry

Faculty, Hirosaki University, Japan

Asst. Prof. UR \& Industry

Industry

Lecturer, U. of Bristol, England 


\section{Publications by Rochester Experiment group 1991 - 2012: A. Bodek, R. Demina, T. Ferbel, S. Manly, K. McFarland, A. Melissinos, S. Olsen, P. Slattery, P. Tipton}

1. "Combined search for the quarks of a sequential fourth generation" S. Chatrchyan et al. [ CMS Collaboration]. arXiv:1209.1062 [hep-ex]

2. "Search for pair produced fourth-generation up-type quarks in pp collisions at $\operatorname{sqrt}(\mathrm{s})=7 \mathrm{TeV}$ with a lepton in the final state"

S. Chatrchyan et al. [ CMS Collaboration].

arXiv:1209.0471 [hep-ex]

3. "Measurements of the Top-quark Mass and the $t \bar{t}$ Cross Section in the Hadronic $\tau+$ Jets Decay Channel at $\sqrt{s}=1.96$ TeV"

T. Aaltonen et al. [CDF Collaboration].

arXiv:1208.5720 [hep-ex]

4. "Search for supersymmetry in events with b-quark jets and missing transverse energy in pp collisions at $7 \mathrm{TeV}$ "

, et al. [CMS Collaboration].

arXiv:1208.4859 [hep-ex]

5. "Extracting Muon Momentum Scale Corrections for Hadron Collider Experiments"

A. Bodek, A. van Dyne, J. Y. Han, W. Sakumoto and A. Strelnikov. arXiv:1208.3710 [hep-ex]

6. "Study of the dijet mass spectrum in $p p \rightarrow W+$ jets events at $\sqrt{s}=7 \mathbf{T e V}$ "

S. Chatrchyan et al. [CMS Collaboration]. arXiv:1208.3477 [hep-ex]

7. "Search for three-jet resonances in pp collisions at $\operatorname{sqrt}(\mathrm{s})=7 \mathrm{TeV}$ "

S. Chatrchyan et al. [CMS Collaboration]. arXiv:1208.2931 [hep-ex]

8. "Observation of sequential Upsilon suppression in $\mathrm{PbPb}$ collisions"

S. Chatrchyan et al. [CMS Collaboration].

arXiv:1208.2826 [nucl-ex]

9. "Measurement of the $\mathrm{t}$ t-bar production cross section in the dilepton channel in pp collisions at $\operatorname{sqrt}(\mathrm{s})=7 \mathrm{TeV}$ "

S. Chatrchyan et al. [CMS Collaboration].

arXiv:1208.2671 [hep-ex] 
10. "Search for the standard model Higgs boson produced in association with top quarks using the full CDF data set"

T. Aaltonen et al. [CDF Collaboration].

arXiv:1208.2662 [hep-ex]

11. "Measurement of the azimuthal anisotropy of neutral pions in $\mathrm{PbPb}$ collisions at $\operatorname{sqrt}(\mathrm{s}(\mathrm{NN}))=2.76 \mathrm{TeV}$ "

S. Chatrchyan et al. [CMS Collaboration].

arXiv:1208.2470 [nucl-ex]

12. "Search for flavor changing neutral currents in top quark decays in pp collisions at 7 TeV"

S. Chatrchyan et al. [CMS Collaboration].

arXiv:1208.0957 [hep-ex]

13. "Search for a W' boson decaying to a bottom quark and a top quark in pp collisions at $\operatorname{sqrt}(\mathrm{s})=7 \mathrm{TeV}$ "

S. Chatrchyan et al. [CMS Collaboration].

arXiv:1208.0956 [hep-ex]

14. "Observation of a new boson at a mass of $125 \mathrm{GeV}$ with the CMS experiment at the LHC"

S. Chatrchyan et al. [CMS Collaboration].

arXiv:1207.7235 [hep-ex]

Phys. Lett. B

15. "Transverse momentum cross section of $e^{+} e^{-}$pairs in the $Z$-boson region from $p \bar{p}$ collisions at $\sqrt{s}=1.96 \mathbf{T e V}^{\prime \prime}$

T. Aaltonen et al. [CDF Collaboration].

arXiv:1207.7138 [hep-ex]

16. "Precision Top-Quark Mass Measurements at CDF"

T. Aaltonen et al. [CDF Collaboration].

arXiv:1207.6758 [hep-ex]

17. "Evidence for a particle produced in association with weak bosons and decaying to a bottom-antibottom quark pair in Higgs boson searches at the Tevatron"

T. Aaltonen et al. [CDF and D0 Collaborations].

arXiv:1207.6436 [hep-ex]

Phys. Rev. Lett. 109, 071804 (2012)

18. "Search for a Higgs boson in the diphoton final state using the full CDF data set from proton-antiproton collisions at $\sqrt{ } s=1.96 \mathrm{TeV}$ '

T. Aaltonen et al. [CDF Collaboration].

arXiv:1207.6386 [hep-ex]

19. "Search for heavy Majorana neutrinos in $\mathbf{m u}+\mathbf{m u}+[\mathrm{mu}-\mathrm{mu}-]$ and $\mathrm{e}+\mathrm{e}+[\mathrm{e}-\mathrm{e}-]$ events in pp collisions at sqrt(s) $=7 \mathrm{TeV}^{\prime \prime}$ 
S. Chatrchyan et al. [CMS Collaboration].

arXiv:1207.6079 [hep-ex]

20. "Search for pair production of first- and second-generation scalar leptoquarks in pp collisions at $\operatorname{sqrt}(\mathrm{s})=7 \mathrm{TeV} "$

S. Chatrchyan et al. [CMS Collaboration].

arXiv:1207.5406 [hep-ex]

21. "An inclusive search for the Higgs boson in the four-lepton final state at CDF" T. Aaltonen et al. [CDF Collaboration].

arXiv:1207.5016 [hep-ex]

22. "Study of the inclusive production of charged pions, kaons, and protons in pp collisions at sqrt(s) $=0.9,2.76$, and $7 \mathrm{TeV}$ "

S. Chatrchyan et al. [CMS Collaboration].

arXiv:1207.4724 [hep-ex]

23. "Forward-backward asymmetry of Drell-Yan lepton pairs in $p p$ collisions at $\sqrt{s}=7$ TeV"

S. Chatrchyan et al. [CMS Collaboration].

arXiv:1207.3973 [hep-ex]

24. "Search for Neutral Higgs Bosons in Events with Multiple Bottom Quarks at the Tevatron"

T. Aaltonen et al. [CDF and D0 Collaborations].

arXiv:1207.2757 [hep-ex]

25. "A search for a doubly-charged Higgs boson in pp collisions at sqrt(s) $=7$ TeV" S. Chatrchyan et al. [CMS Collaboration].

arXiv:1207.2666 [hep-ex]

26. "Measurement of the underlying event activity in $p p$ collisions at $\sqrt{s}=0.9$ and 7 TeV with the novel jet-area/median approach"

S. Chatrchyan et al. [CMS Collaboration].

arXiv:1207.2392 [hep-ex]

27. "Measurement of the difference of CP-violating asymmetries in $D^{0} \rightarrow K^{+} K^{-}$and $D^{0} \rightarrow \pi^{+} \pi^{-}$decays at CDF"

T. Aaltonen et al. [CDF Collaboration].

arXiv:1207.2158 [hep-ex]

28. "Search for new physics in the multijet and missing transverse momentum final state in proton-proton collisions at $\sqrt{s}=7 \mathrm{TeV}$ "

S. Chatrchyan et al. [CMS Collaboration].

arXiv:1207.1898 [hep-ex]

29. "Search for supersymmetry in hadronic final states using MT2 in $p p$ collisions at $\sqrt{s}=7 \mathbf{T e V}^{\prime \prime}$ 
S. Chatrchyan et al. [CMS Collaboration].

arXiv:1207.1798 [hep-ex]

30. "Search for the standard model Higgs boson decaying to a $b \bar{b}$ pair in events with no charged leptons and large missing transverse energy using the full CDF data set"

T. Aaltonen et al. [CDF Collaboration].

arXiv:1207.1711 [hep-ex]

31. "Combined search for the standard model Higgs boson decaying to a bb pair using the full CDF data set"

T. Aaltonen et al. [CDF Collaboration].

arXiv:1207.1707 [hep-ex]

32. "Search for the standard model Higgs boson decaying to a bb pair in events with two oppositely-charged leptons using the full CDF data set"

T. Aaltonen et al. [CDF Collaboration].

arXiv:1207.1704 [hep-ex]

33. "Search for the standard model Higgs boson decaying to a bb pair in events with one charged lepton and large missing transverse energy using the full CDF data set"

T. Aaltonen et al. [CDF Collaboration].

arXiv:1207.1703 [hep-ex]

34. "Extraction of Neutrino Flux with the Low $\nu$ Method at MiniBooNE Energies" A. Bodek, U. Sarica, K. S. Kuzmin and V. A. Naumov.

arXiv:1207.1247 [hep-ex]

35. "Search for a fermiophobic Higgs boson in $p p$ collisions at $\sqrt{s}=7$ TeV"

S. Chatrchyan et al. [CMS Collaboration].

arXiv:1207.1130 [hep-ex]

36. "Combination of the top-quark mass measurements from the Tevatron collider" T. Aaltonen et al. [CDF and D0 Collaborations].

arXiv:1207.1069 [hep-ex]

37. "Measurement of CP-violation asymmetries in $D^{0} \rightarrow K_{S} \pi^{+} \pi^{-}$"

T. Aaltonen et al. [CDF Collaboration].

arXiv:1207.0825 [hep-ex]

Phys. Rev. D 86, 032007 (2012)

38. "Search for new physics with long-lived particles decaying to photons and missing energy in pp collisions at $\operatorname{sqrt}(\mathrm{s})=7 \mathrm{TeV}^{\prime \prime}$

S. Chatrchyan et al. [CMS Collaboration].

arXiv:1207.0627 [hep-ex]

39. "Search for stopped long-lived particles produced in $p p$ collisions at $\sqrt{s}=7$ TeV" S. Chatrchyan et al. [CMS Collaboration]. 
arXiv:1207.0106 [hep-ex]

JHEP 1208, 026 (2012)

40. "Inclusive and differential measurements of the $t \bar{t}$ charge asymmetry in protonproton collisions at $7 \mathrm{TeV}$ "

S. Chatrchyan et al. [CMS Collaboration].

arXiv:1207.0065 [hep-ex]

41. "Search for a light pseudoscalar Higgs boson in the dimuon decay channel in pp collisions at $\operatorname{sqrt}(\mathrm{s})=7 \mathrm{TeV}$ "

S. Chatrchyan et al. [CMS Collaboration].

arXiv:1206.6326 [hep-ex]

42. "Search for dark matter and large extra dimensions in monojet events in pp collisions at $\operatorname{sqrt}(\mathrm{s})=7 \mathrm{TeV}$ "

S. Chatrchyan et al. [CMS Collaboration].

arXiv:1206.5663 [hep-ex]

43. "Search for the standard model Higgs boson produced in association with a $W^{ \pm}$ boson with $7.5 \mathrm{fb}^{-1}$ integrated luminosity at CDF"

T. Aaltonen et al. [CDF Collaboration].

arXiv:1206.5063 [hep-ex]

Phys. Rev. D 86, 032011 (2012)

44. "Performance of CMS muon reconstruction in pp collision events at $\operatorname{sqrt}(\mathrm{s})=7$ TeV"

S. Chatrchyan et al. [CMS Collaboration].

arXiv:1206.4071 [physics.ins-det]

45. "Diffractive Dijet Production in $\bar{p} p$ Collisions at $\sqrt{s}=1.96 \mathrm{TeV}$ "

T. Aaltonen et al. [CDF Collaboration].

arXiv:1206.3955 [hep-ex]

Phys. Rev. D 86, 032009 (2012)

46. "Search for new physics in events with opposite-sign leptons, jets, and missing transverse energy in pp collisions at $\operatorname{sqrt}(\mathrm{s})=7 \mathrm{TeV}$ "

S. Chatrchyan et al. [CMS Collaboration]. arXiv:1206.3949 [hep-ex]

47. "Search for charge-asymmetric production of $\mathbf{W}$ ' bosons in top pair + jet events from pp collisions at sqrt(s) $=7 \mathrm{TeV}$ "

S. Chatrchyan et al. [CMS Collaboration].

arXiv:1206.3921 [hep-ex]

48. "Measurement of the electron charge asymmetry in inclusive $\mathbf{W}$ production in pp collisions at sqrt(s) $=7 \mathrm{TeV}$ "

S. Chatrchyan et al. [CMS Collaboration].

arXiv:1206.2598 [hep-ex] 
49. "Search for narrow resonances in dilepton mass spectra in pp collisions at sqrt(s) $=7 \mathrm{TeV}$ "

S. Chatrchyan et al. [CMS Collaboration].

arXiv:1206.1849 [hep-ex]

Phys. Lett. B 714, 158 (2012)

50. "Search for high mass resonances decaying into tau-lepton pairs in pp collisions at $\operatorname{sqrt}(\mathrm{s})=7 \mathrm{TeV} "$

S. Chatrchyan et al. [CMS Collaboration].

arXiv:1206.1725 [hep-ex]

51. "Search for exotic particles decaying to $\mathrm{WZ}$ in pp collisions at $\operatorname{sqrt}(\mathrm{s})=7 \mathrm{TeV}$ " S. Chatrchyan et al. [CMS Collaboration].

arXiv:1206.0433 [hep-ex]

52. "Search for new physics with same-sign isolated dilepton events with jets and missing transverse energy"

S. Chatrchyan et al. [CMS Collaboration].

arXiv:1205.6615 [hep-ex]

Phys. Rev. Lett. 109, 071803 (2012)

53. "Study of $\mathrm{W}$ boson production in $\mathrm{PbPb}$ and $\mathrm{pp}$ collisions at $\operatorname{sqrt}(\mathrm{s}[\mathrm{NN}])=2.76$ TeV"

S. Chatrchyan et al. [CMS Collaboration].

arXiv:1205.6334 [nucl-ex]

Phys. Lett. B 715, 66 (2012)

54. "Measurement of jet fragmentation into charged particles in $\mathrm{pp}$ and $\mathrm{PbPb}$ collisions at $\operatorname{sqrt}(\mathrm{s}[\mathrm{NN}])=2.76 \mathrm{TeV} "$

S. Chatrchyan et al. [CMS Collaboration].

arXiv:1205.5872 [nucl-ex]

55. "Search for a light charged Higgs boson in top quark decays in pp collisions at $\operatorname{sqrt}(\mathrm{s})=7 \mathrm{TeV}^{\prime \prime}$

S. Chatrchyan et al. [CMS Collaboration].

arXiv:1205.5736 [hep-ex]

JHEP 1207, 143 (2012)

56. "Search for new physics in events with same-sign dileptons and b-tagged jets in pp collisions at sqrt(s) $=7 \mathrm{TeV}$ "

S. Chatrchyan et al. [CMS Collaboration].

arXiv:1205.3933 [hep-ex]

57. "Fundamental Physics at the Intensity Frontier"

J. L. Hewett, H. Weerts, R. Brock, J. N. Butler, B. C. K. Casey, J. Collar, A. de Govea and R. Essig et al..

arXiv:1205.2671 [hep-ex] 
58. "Measurement of the pseudorapidity and centrality dependence of the transverse energy density in $\mathrm{PbPb}$ collisions at $\operatorname{sqrt}(\mathrm{s}[\mathrm{NN}])=2.76 \mathrm{TeV}$ "

S. Chatrchyan et al. [CMS Collaboration].

arXiv:1205.2488 [nucl-ex]

59. "Measurement of the Lambda(b) cross section and the anti-Lambda(b) to Lambda(b) ratio with Lambda(b) to $\mathrm{J} /$ Psi Lambda decays in pp collisions at sqrt(s) $=7$ TeV"

S. Chatrchyan et al. [CMS Collaboration].

arXiv:1205.0594 [hep-ex]

Phys. Lett. B 714, 136 (2012)

60. " $W$ boson polarization measurement in the $t \bar{t}$ dilepton channel using the CDF II Detector"

T. Aaltonen et al. [CDF Collaboration].

arXiv:1205.0354 [hep-ex]

61. "Search for heavy long-lived charged particles in pp collisions at $\operatorname{sqrt}(\mathrm{s})=7 \mathrm{TeV}$ " S. Chatrchyan et al. [CMS Collaboration].

arXiv:1205.0272 [hep-ex]

Phys. Lett. B 713, 408 (2012)

62. "Studies of jet quenching using isolated-photon+jet correlations in $\mathrm{PbPb}$ and pp collisions at $\operatorname{sqrt}(\mathrm{s}[\mathrm{NN}])=2.76 \mathrm{TeV}^{\prime \prime}$

S. Chatrchyan et al. [CMS Collaboration]. arXiv:1205.0206 [nucl-ex]

63. "Observation of a new $\mathrm{Xi}(\mathrm{b})$ baryon"

S. Chatrchyan et al. [CMS Collaboration]. arXiv:1204.5955 [hep-ex]

Phys. Rev. Lett. 108, 252002 (2012)

64. "Search for anomalous production of multilepton events in pp collisions at sqrt(s)=7 TeV"

S. Chatrchyan et al. [CMS Collaboration].

arXiv:1204.5341 [hep-ex]

JHEP 1206, 169 (2012)

65. "Search for leptonic decays of $\mathrm{W}$ ' bosons in pp collisions at $\operatorname{sqrt}(\mathrm{s})=7 \mathrm{TeV}$ "

S. Chatrchyan et al. [CMS Collaboration].

arXiv:1204.4764 [hep-ex]

JHEP 1208, 023 (2012)

66. "Search for physics beyond the standard model in events with a $\mathrm{Z}$ boson, jets, and missing transverse energy in pp collisions at $\operatorname{sqrt}(\mathrm{s})=7 \mathrm{TeV}$ "

S. Chatrchyan et al. [CMS Collaboration].

arXiv:1204.3774 [hep-ex] 
67. "Shape, transverse size, and charged hadron multiplicity of jets in pp collisions at 7 TeV"

S. Chatrchyan et al. [CMS Collaboration].

arXiv:1204.3170 [hep-ex]

JHEP 1206, 160 (2012)

68. "Measurement of the mass difference between top and antitop quarks"

S. Chatrchyan et al. [CMS Collaboration].

arXiv:1204.2807 [hep-ex]

JHEP 1206, 109 (2012)

69. "Search for anomalous $\mathbf{t} \mathbf{t}$-bar production in the highly-boosted all-hadronic final state"

S. Chatrchyan et al. [CMS Collaboration].

arXiv:1204.2488 [hep-ex]

70. "Azimuthal anisotropy of charged particles at high transverse momenta in $\mathrm{PbPb}$ collisions at $\operatorname{sqrt}(\mathrm{s}[\mathrm{NN}])=2.76 \mathrm{TeV}$ "

S. Chatrchyan et al. [CMS Collaboration].

arXiv:1204.1850 [nucl-ex]

Phys. Rev. Lett. 109, 022301 (2012)

71. "Measurement of the $\mathrm{Z} /$ gamma* $+\mathrm{b}$-jet cross section in pp collisions at $7 \mathrm{TeV}$ " S. Chatrchyan et al. [CMS Collaboration].

arXiv:1204.1643 [hep-ex]

JHEP 1206, 126 (2012)

72. "Measurement of the elliptic anisotropy of charged particles produced in $\mathrm{PbPb}$ collisions at nucleon-nucleon center-of-mass energy $=2.76 \mathrm{TeV} "$

S. Chatrchyan et al. [CMS Collaboration].

arXiv:1204.1409 [nucl-ex]

73. "Measurement of the underlying event in the Drell-Yan process in proton-proton collisions at sqrt(s) $=7$ TeV"

S. Chatrchyan et al. [CMS Collaboration].

arXiv:1204.1411 [hep-ex]

74. "Search for heavy bottom-like quarks in 4.9 inverse femtobarns of pp collisions at $\operatorname{sqrt}(\mathrm{s})=7 \mathrm{TeV}^{\prime \prime}$

S. Chatrchyan et al. [CMS Collaboration].

arXiv:1204.1088 [hep-ex]

JHEP 1205, 123 (2012)

75. "Search for Dark Matter and Large Extra Dimensions in pp Collisions Yielding a Photon and Missing Transverse Energy"

S. Chatrchyan et al. [CMS Collaboration].

arXiv:1204.0821 [hep-ex]

Phys. Rev. Lett. 108, 261803 (2012) 
76. "Ratios of dijet production cross sections as a function of the absolute difference in rapidity between jets in proton-proton collisions at $\operatorname{sqrt}(\mathrm{s})=7 \mathrm{TeV}$ "

S. Chatrchyan et al. [CMS Collaboration].

arXiv:1204.0696 [hep-ex]

77. "Measurement of $B_{s}^{0} \rightarrow D_{s}^{(*)+} D_{s}^{(*)-}$ Branching Ratios"

T. Aaltonen et al. [CDF Collaboration].

arXiv:1204.0536 [hep-ex]

Phys. Rev. Lett. 108, 201801 (2012)

78. "Measurement of the top quark pair production cross section in $p p$ collisions at $\sqrt{s}=7 \mathrm{TeV}$ in dilepton final states containing a $\tau^{\prime \prime}$

S. Chatrchyan et al. [CMS Collaboration].

arXiv:1203.6810 [hep-ex]

Phys. Rev. D 85, 112007 (2012)

79. "Search for the Standard Model Higgs Boson Produced in Association with a $Z$ Boson in $p \bar{p}$ Collisions at $\sqrt{s}=1.96$ TeV"

T. Aaltonen et al. [CDF Collaboration].

arXiv:1203.5815 [hep-ex]

80. "Search for heavy, top-like quark pair production in the dilepton final state in $p p$ collisions at $\sqrt{s}=7 \mathrm{TeV}^{\prime \prime}$

S. Chatrchyan et al. [CMS Collaboration].

arXiv:1203.5410 [hep-ex]

81. "R \& D Results on Scintillating Tile / Fiber Calorimetry for the CDF and SDC Detectors"

P. d. Barbaro, A. Bodek, H. S. Budd, Q. Fan, W. K. Sakumoto, B. Winer, J. Proudfoot and D. G. Underwood et al..

82. "Search for Scalar Top Quark Production in $p \bar{p}$ Collisions at $\sqrt{s}=1.96$ TeV" T. Aaltonen et al. [CDF Collaboration].

arXiv:1203.4171 [hep-ex]

83. "Search for $B_{s}^{0} t o \mu^{+} \mu^{-}$and $B^{0} t o \mu^{+} \mu^{-}$decays"

S. Chatrchyan et al. [CMS Collaboration].

arXiv:1203.3976 [hep-ex]

JHEP 1204, 033 (2012)

84. "Search for a heavy particle decaying to a top quark and a light quark in $p \bar{p}$ collisions at $\sqrt{s}=1.96 \mathrm{TeV}$ "

T. Aaltonen et al. [CDF Collaboration].

arXiv:1203.3894 [hep-ex]

Phys. Rev. Lett. 108, 211805 (2012)

85. "Measurement of the cross section for production of $\mathbf{b}$ b-bar $\mathbf{X}$, decaying to muons in pp collisions at $\operatorname{sqrt}(\mathrm{s})=7 \mathrm{TeV}$ " 
S. Chatrchyan et al. [CMS Collaboration].

arXiv:1203.3458 [hep-ex]

JHEP 1206, 110 (2012)

86. "Demonstration of Communication using Neutrinos"

D. D. Stancil, P. Adamson, M. Alania, L. Aliaga, M. Andrews, C. Araujo Del Castillo, L. Bagby and J. L. Bazo Alba et al..

arXiv:1203.2847 [hep-ex]

Mod. Phys. Lett. A 27, 1250077 (2012)

87. "A Search for dark matter in events with one jet and missing transverse energy in $p \bar{p}$ collisions at $\sqrt{s}=1.96 \mathbf{T e V}$ "

T. Aaltonen et al. [CDF Collaboration].

arXiv:1203.0742 [hep-ex]

Phys. Rev. Lett. 108, 211804 (2012)

88. "Precise measurement of the $W$-boson mass with the CDF II detector"

T. Aaltonen et al. [CDF Collaboration].

arXiv:1203.0275 [hep-ex]

Phys. Rev. Lett. 108, 151803 (2012)

89. "Measurement of the $W Z$ Cross Section and Triple Gauge Couplings in $p \bar{p}$ Collisions at $\sqrt{s}=1.96 \mathrm{TeV}^{\prime}$

T. Aaltonen et al. [CDF Collaboration].

arXiv:1202.6629 [hep-ex]

Phys. Rev. D 86, 031104 (2012)

90. "Search for microscopic black holes in pp collisions at $\operatorname{sqrt}(\mathrm{s})=7 \mathrm{TeV}$ "

S. Chatrchyan et al. [CMS Collaboration].

arXiv:1202.6396 [hep-ex]

JHEP 1204, 061 (2012)

91. "Search for a dark matter candidate produced in association with a single top quark in $p \bar{p}$ collisions at $\sqrt{s}=1.96 \mathrm{TeV}$ "

T. Aaltonen et al. [CDF Collaboration].

arXiv:1202.5653 [hep-ex]

Phys. Rev. Lett. 108, 201802 (2012)

92. "Search for quark compositeness in dijet angular distributions from pp collisions at $\operatorname{sqrt}(\mathrm{s})=7 \mathrm{TeV}$ "

S. Chatrchyan et al. [CMS Collaboration].

arXiv:1202.5535 [hep-ex]

JHEP 1205, 055 (2012)

93. "Combination of CDF and Do measurements of the $W$ boson helicity in top quark decays"

T. Aaltonen et al. [CDF and D0 Collaborations].

arXiv:1202.5272 [hep-ex] 
94. "Jet momentum dependence of jet quenching in $\mathrm{PbPb}$ collisions at $\operatorname{sqrt}(\mathrm{sNN})=\mathbf{2 . 7 6}$ TeV"

S. Chatrchyan et al. [CMS Collaboration].

arXiv:1202.5022 [nucl-ex]

Phys. Lett. B 712, 176 (2012)

95. "Inclusive b-jet production in pp collisions at sqrt(s)=7 $\mathrm{TeV}$ "

S. Chatrchyan et al. [CMS Collaboration].

arXiv:1202.4617 [hep-ex]

JHEP 1204, 084 (2012)

96. "Search for the standard model Higgs boson decaying to bottom quarks in pp collisions at $\operatorname{sqrt}(\mathrm{s})=7 \mathrm{TeV}$ "

S. Chatrchyan et al. [CMS Collaboration].

arXiv:1202.4195 [hep-ex]

Phys. Lett. B 710, 284 (2012)

97. "Search for neutral Higgs bosons decaying to tau pairs in pp collisions at sqrt(s)=7 TeV"

S. Chatrchyan et al. [CMS Collaboration].

arXiv:1202.4083 [hep-ex]

Phys. Lett. B 713, 68 (2012)

98. "Search for large extra dimensions in dimuon and dielectron events in pp collisions at $\sqrt{s}=7 \mathrm{TeV}^{\prime \prime}$

S. Chatrchyan et al. [CMS Collaboration].

arXiv:1202.3827 [hep-ex]

Phys. Lett. B 711, 15 (2012)

99. "Search for the standard model Higgs boson in the $\mathbf{H}$ to $\mathrm{ZZ}$ to $212 \mathrm{nu}$ channel in pp collisions at sqrt(s) $=7 \mathrm{TeV}$ "

S. Chatrchyan et al. [CMS Collaboration].

arXiv:1202.3478 [hep-ex]

JHEP 1203, 040 (2012)

100. "Search for the standard model Higgs boson in the $\mathbf{H}$ to $\mathrm{ZZ}$ to $\mathrm{ll}$ tau tau decay channel in pp collisions at $\operatorname{sqrt}(\mathrm{s})=7 \mathrm{TeV}$ "

S. Chatrchyan et al. [CMS Collaboration].

arXiv:1202.3617 [hep-ex]

JHEP 1203, 081 (2012)

101. "Study of high-pT charged particle suppression in $\mathrm{PbPb}$ compared to pp collisions at $\operatorname{sqrt}(\mathrm{sNN})=2.76 \mathrm{TeV}$ "

S. Chatrchyan et al. [CMS Collaboration].

arXiv:1202.2554 [nucl-ex]

Eur. Phys. J. C 72, 1945 (2012) 
102. "Search for the standard model Higgs boson in the decay channel $\mathrm{H}$ to $\mathrm{ZZ}$ to 4 leptons in pp collisions at sqrt(s) $=7 \mathrm{TeV}$ "

S. Chatrchyan et al. [CMS Collaboration].

arXiv:1202.1997 [hep-ex]

Phys. Rev. Lett. 108, 111804 (2012)

103. "Search for the standard model Higgs boson decaying to a $\mathrm{W}$ pair in the fully leptonic final state in pp collisions at $\operatorname{sqrt}(\mathrm{s})=7 \mathrm{TeV}$ "

S. Chatrchyan et al. [CMS Collaboration].

arXiv:1202.1489 [hep-ex]

Phys. Lett. B 710, 91 (2012)

104. "Combined results of searches for the standard model Higgs boson in pp collisions at $\operatorname{sqrt}(\mathrm{s})=7 \mathrm{TeV} "$

S. Chatrchyan et al. [CMS Collaboration].

arXiv:1202.1488 [hep-ex]

Phys. Lett. B 710, 26 (2012)

105. "Search for the standard model Higgs boson decaying into two photons in pp collisions at $\operatorname{sqrt}(\mathrm{s})=7 \mathrm{TeV}$ "

S. Chatrchyan et al. [CMS Collaboration].

arXiv:1202.1487 [hep-ex]

Phys. Lett. B 710, 403 (2012)

106. "Search for a Higgs boson in the decay channel $\mathbf{H}$ to $\mathrm{ZZ}\left(^{*}\right)$ to $\mathbf{q}$ qbar $\mathbf{l}-1+$ in $\mathrm{pp}$ collisions at $\operatorname{sqrt}(\mathrm{s})=7 \mathrm{TeV}^{\prime \prime}$

S. Chatrchyan et al. [CMS Collaboration].

arXiv:1202.1416 [hep-ex]

JHEP 1204, 036 (2012)

107. "Search for anomalous production of multiple leptons in association with $W$ and $Z$ bosons at CDF"

T. Aaltonen et al. [CDF Collaboration].

arXiv:1202.1260 [hep-ex]

Phys. Rev. D 85, 092001 (2012)

108. "Measurement of the inclusive production cross sections for forward jets and for dijet events with one forward and one central jet in pp collisions at $\operatorname{sqrt}(s)=7$ TeV"

S. Chatrchyan et al. [CMS Collaboration].

arXiv:1202.0704 [hep-ex]

JHEP 1206, 036 (2012)

109. "Suppression of non-prompt $\mathbf{J} / \mathbf{p s i}$, prompt $\mathbf{J} / \mathbf{p s i}$, and $\mathbf{Y}(\mathbf{1 S})$ in $\mathbf{P b P b}$ collisions at $\operatorname{sqrt}(\mathrm{sNN})=2.76 \mathrm{TeV}^{\prime \prime}$

S. Chatrchyan et al. [CMS Collaboration].

arXiv:1201.5069 [nucl-ex]

JHEP 1205, 063 (2012) 
110. "Search for a low mass Standard Model Higgs boson in the $\tau-\tau$ decay channel in $p \bar{p}$ collisions at $\sqrt{s}=1.96 \mathrm{TeV}$ "

T. Aaltonen et al. [CDF Collaboration].

arXiv:1201.4880 [hep-ex]

Phys. Rev. Lett. 108, 181804 (2012)

111. "Centrality dependence of dihadron correlations and azimuthal anisotropy harmonics in $\mathrm{PbPb}$ collisions at $\operatorname{sqrt}(\mathrm{s}[\mathrm{NN}])=2.76 \mathrm{TeV}$ "

S. Chatrchyan et al. [CMS Collaboration].

arXiv:1201.3158 [nucl-ex]

Eur. Phys. J. C 72, 2012 (2012)

112. "Measurement of isolated photon production in pp and $\mathrm{PbPb}$ collisions at sqrt(sNN) $=2.76 \mathrm{TeV}$ "

S. Chatrchyan et al. [CMS Collaboration].

arXiv:1201.3093 [nucl-ex]

Phys. Lett. B 710, 256 (2012)

113. "Methods to Determine Neutrino Flux at Low Energies:Investigation of the Low $\nu$ Method"

A. Bodek, U. Sarica, D. Naples and L. Ren.

arXiv:1201.3025 [hep-ex]

Eur. Phys. J. C 72, 1973 (2012)

114. "Measurement of the charge asymmetry in top-quark pair production in protonproton collisions at sqrt(s) $=7 \mathrm{TeV}$ "

S. Chatrchyan et al. [CMS Collaboration].

arXiv:1112.5100 [hep-ex]

Phys. Lett. B 709, 28 (2012)

115. "Measurement of the Top Quark Mass in the All-Hadronic Mode at CDF"

T. Aaltonen et al. [CDF Collaboration].

arXiv:1112.4891 [hep-ex]

Phys. Lett. B 714, 24 (2012)

116. "Search for Standard Model Higgs Boson Production in Association with a $W$ Boson Using a Matrix Element Technique at CDF in $p \bar{p}$ Collisions at $\sqrt{s}=1.96$ TeV"

T. Aaltonen et al. [CDF Collaboration].

arXiv:1112.4358 [hep-ex]

Phys. Rev. D 85, 072001 (2012)

117. "Measurement of the branching fraction $\mathcal{B}\left(\Lambda_{b}^{0} \rightarrow \Lambda_{c}^{+} \pi^{-} \pi^{+} \pi^{-}\right)$at CDF"

T. Aaltonen et al. [CDF Collaboration].

arXiv:1112.3334 [hep-ex]

Phys. Rev. D 85, 032003 (2012) 
118. "Measurement of the masses and widths of the bottom baryons $\Sigma_{b}^{+-}$and $\Sigma_{b}^{*+-}$ " T. Aaltonen et al. [CDF Collaboration].

arXiv:1112.2808 [hep-ex]

Phys. Rev. D 85, 092011 (2012)

119. "Measurement of $Z Z$ production in leptonic final states at $\sqrt{ } s$ of $1.96 \mathrm{TeV}$ at CDF"

T. Aaltonen et al. [CDF Collaboration].

arXiv:1112.2978 [hep-ex]

Phys. Rev. Lett. 108, 101801 (2012)

120. "Search for Standard Model Higgs Boson Production in Association with a $W$ Boson at CDF"

T. Aaltonen et al. [CDF Collaboration].

arXiv:1112.1930 [hep-ex]

Phys. Rev. D 85, 052002 (2012)

121. "Measurement of the CP-Violating Phase $\beta_{s}^{J / \Psi \phi}$ in $B_{s}^{0} \rightarrow J / \Psi \phi$ Decays with the CDF II Detector"

T. Aaltonen et al. [CDF Collaboration].

arXiv:1112.1726 [hep-ex]

Phys. Rev. D 85, 072002 (2012)

122. "Measurements of Angular Distributions of Muons From $\Upsilon$ Meson Decays in $p \bar{p}$ Collisions at $\sqrt{s}=1.96 \mathrm{TeV}$ "

T. Aaltonen et al. [CDF Collaboration].

arXiv:1112.1591 [hep-ex]

Phys. Rev. Lett. 108, 151802 (2012)

123. "Search for new phenomena in events with two $Z$ bosons and missing transverse momentum in $p \bar{p}$ collisions at $\sqrt{s}=1.96 \mathrm{TeV}^{\prime \prime}$

T. Aaltonen et al. [CDF Collaboration].

arXiv:1112.1577 [hep-ex]

Phys. Rev. D 85, 011104 (2012)

124. "Observation of Exclusive Gamma Gamma Production in $p \bar{p}$ Collisions at $\sqrt{s}=$ 1.96 TeV"

T. Aaltonen et al. [CDF Collaboration].

arXiv:1112.0858 [hep-ex]

Phys. Rev. Lett. 108, 081801 (2012)

125. "Search for signatures of extra dimensions in the diphoton mass spectrum at the Large Hadron Collider"

S. Chatrchyan et al. [CMS Collaboration].

arXiv:1112.0688 [hep-ex]

126. "Exclusive photon-photon production of muon pairs in proton-proton collisions at $\operatorname{sqrt}(\mathrm{s})=7 \mathrm{TeV}^{\prime \prime}$ 
S. Chatrchyan et al. [CMS Collaboration].

arXiv:1111.5536 [hep-ex]

JHEP 1201, 052 (2012)

127. "Measurement of $\mathbf{C P}$-violating asymmetries in $D^{0} \rightarrow \pi^{+} \pi^{-}$and $D^{0} \rightarrow K^{+} K^{-}$ decays at CDF"

T. Aaltonen et al. [CDF Collaboration].

arXiv:1111.5023 [hep-ex]

Phys. Rev. D 85, 012009 (2012)

128. "The T2K ND280 Off-Axis Pi-Zero Detector"

S. Assylbekov, B. E. Berger, H. Berns, D. Beznosko, A. Bodek, R. Bradford, N. Buchanan and H. Budd et al..

arXiv:1111.5030 [physics.ins-det]

Nucl. Instrum. Meth. A 686, 48 (2012)

129. "An additional study of multi-muon events produced in $p \bar{p}$ collisions at $\sqrt{s}=1.96$ TeV"

T. Aaltonen et al. [CDF Collaboration].

arXiv:1111.5242 [hep-ex]

Phys. Lett. B 710, 278 (2012)

130. "Search for high-mass resonances decaying into $Z Z$ in $\mathbf{p} \bar{p}$ collisions at $\sqrt{s}=$ 1.96 TeV"

T. Aaltonen et al. [CDF Collaboration].

arXiv:1111.3432 [hep-ex]

Phys. Rev. D 85, 012008 (2012)

131. "J/psi and $\mathrm{psi}(2 \mathrm{~S})$ production in $\mathrm{pp}$ collisions at $\operatorname{sqrt}(\mathrm{s})=7 \mathrm{TeV} "$

S. Chatrchyan et al. [CMS Collaboration].

arXiv:1111.1557 [hep-ex]

JHEP 1202, 011 (2012)

132. "Evidence for the charmless annihilation decay mode $B_{s}^{0} \rightarrow \pi^{+} \pi^{-}$"

T. Aaltonen et al. [CDF Collaboration].

arXiv:1111.0485 [hep-ex]

Phys. Rev. Lett. 108, 211803 (2012)

133. "Measurement of the Production Cross Section for Pairs of Isolated Photons in pp collisions at sqrt(s) $=7 \mathrm{TeV}$ "

S. Chatrchyan et al. [CMS Collaboration].

arXiv:1110.6461 [hep-ex]

JHEP 1201, 133 (2012)

134. "Measurement of the Rapidity and Transverse Momentum Distributions of $\mathrm{Z}$ Bosons in pp Collisions at sqrt(s)=7 TeV"

S. Chatrchyan et al. [CMS Collaboration]. 
arXiv:1110.4973 [hep-ex]

Phys. Rev. D 85, 032002 (2012)

135. "Jet Production Rates in Association with $\mathbf{W}$ and $\mathrm{Z}$ Bosons in pp Collisions at $\operatorname{sqrt}(\mathrm{s})=7 \mathrm{TeV} "$

S. Chatrchyan et al. [CMS Collaboration].

arXiv:1110.3226 [hep-ex]

JHEP 1201, 010 (2012)

136. "Measurement of the weak mixing angle with the Drell-Yan process in protonproton collisions at the LHC"

S. Chatrchyan et al. [CMS Collaboration].

arXiv:1110.2682 [hep-ex]

Phys. Rev. D 84, 112002 (2011)

137. "Measurement of energy flow at large pseudorapidities in $p p$ collisions at $\sqrt{s}=0.9$ and 7 TeV"

S. Chatrchyan et al. [CMS Collaboration].

arXiv:1110.0211 [hep-ex]

JHEP 1111, 148 (2011), [Erratum-ibid. 1202, 055 (2012)]

138. "Forward Energy Flow, Central Charged-Particle Multiplicities, and Pseudorapidity Gaps in $\mathrm{W}$ and $\mathrm{Z}$ Boson Events from pp Collisions at $7 \mathrm{TeV}$ "

S. Chatrchyan et al. [CMS Collaboration].

arXiv:1110.0181 [hep-ex]

Eur. Phys. J. C 72, 1839 (2012)

139. "Performance of tau-lepton reconstruction and identification in CMS"

C. Collaboration et al. [CMS Collaboration].

arXiv:1109.6034 [physics.ins-det]

JINST 7, P01001 (2012)

140. "Letter of Intent to build an Off-axis Detector to study numu to nue oscillations with the NuMI Neutrino Beam"

D. Ayres, G. Drake, M. Goodman, V. Guarino, T. Joffe-Minor, D. Reyna, R. Talaga and J. Thron et al.

hep-ex/0210005

141. "Search for a Vector-like Quark with Charge 2/3 in $t+Z$ Events from pp Collisions at $\operatorname{sqrt}(\mathrm{s})=7 \mathrm{TeV}$ "

S. Chatrchyan et al. [CMS Collaboration].

arXiv:1109.4985 [hep-ex]

Phys. Rev. Lett. 107, 271802 (2011)

142. "Search for a Higgs Boson in the Diphoton Final State in $p \bar{p}$ Collisions at $\sqrt{s}=1.96$ TeV"

T. Aaltonen et al. [CDF Collaboration]. 
arXiv:1109.4427 [hep-ex]

Phys. Rev. Lett. 108, 011801 (2012)

143. "Search for heavy metastable particles decaying to jet pairs in $p \bar{p}$ collisions at $\sqrt{s}=1.96$ TeV"

T. Aaltonen et al. [CDF Collaboration].

arXiv:1109.3136 [hep-ex]

Phys. Rev. D 85, 012007 (2012)

144. "Search for Supersymmetry at the LHC in Events with Jets and Missing Transverse Energy"

S. Chatrchyan et al. [CMS Collaboration].

arXiv:1109.2352 [hep-ex]

Phys. Rev. Lett. 107, 221804 (2011)

145. "Top-quark mass measurement using events with missing transverse energy and jets at CDF"

T. Aaltonen et al. [CDF Collaboration].

arXiv:1109.1490 [hep-ex]

Phys. Rev. Lett. 107, 232002 (2011)

146. "Measurements of branching fraction ratios and CP-asymmetries in suppressed $B^{-} \rightarrow D\left(\rightarrow K^{+} \pi^{-}\right) K^{-}$and $B^{-} \rightarrow D\left(\rightarrow K^{+} \pi^{-}\right) \pi^{-}$decays"

T. Aaltonen et al. [CDF Collaboration].

arXiv:1108.5765 [hep-ex]

Phys. Rev. D 84, 091504 (2011)

147. "Search for resonant production of $t \bar{t}$ decaying to jets in $p \bar{p}$ collisions at $\sqrt{s}=1.96$ TeV"

T. Aaltonen et al. [CDF Collaboration].

arXiv:1108.4755 [hep-ex]

Phys. Rev. D 84, 072003 (2011)

148. "Measurement of the $t \bar{t}$ Production Cross Section in $p p$ Collisions at $\mathbf{7}$ TeV in Lepton + Jets Events Using b-quark Jet Identification"

S. Chatrchyan et al. [CMS Collaboration].

arXiv:1108.3773 [hep-ex]

Phys. Rev. D 84, 092004 (2011)

149. "Measurement of the Differential Cross Section for Isolated Prompt Photon Production in pp Collisions at 7 TeV"

S. Chatrchyan et al. [CMS Collaboration].

arXiv:1108.2044 [hep-ex]

Phys. Rev. D 84, 052011 (2011)

150. "Search for $W Z+Z Z$ production with MET + jets with $b$ enhancement at $\sqrt{s}=$ $1.96 \mathrm{TeV}$ "

T. Aaltonen et al. [CDF Collaboration]. 
arXiv:1108.2060 [hep-ex]

Phys. Rev. D 85, 012002 (2012)

151. "Measurement of the top-quark mass in the lepton+jets channel using a matrix element technique with the CDF II detector"

T. Aaltonen et al. [CDF Collaboration].

arXiv:1108.1601 [hep-ex]

Phys. Rev. D 84, 071105 (2011)

152. "Resolving the Axial Mass Anomaly in neutrino Scattering"

A. Bodek, H. S. Budd and M. E. Christy.

arXiv:1108.0903 [nucl-ex]

AIP Conf. Proc. 1441, 420 (2012)

153. "Measurements of the Angular Distributions in the Decays $B \rightarrow K^{(*)} \mu^{+} \mu^{-}$at CDF"

T. Aaltonen et al. [CDF Collaboration].

arXiv:1108.0695 [hep-ex]

Phys. Rev. Lett. 108, 081807 (2012)

154. "Measurement of the Drell-Yan Cross Section in pp Collisions at sqrt(s) $=7$ TeV"

S. Chatrchyan et al. [CMS Collaboration].

arXiv:1108.0566 [hep-ex]

JHEP 1110, 007 (2011)

155. "Search for new physics in high $p_{T}$ like-sign dilepton events at CDF II"

T. Aaltonen et al. [CDF Collaboration].

arXiv:1108.0101 [hep-ex]

Phys. Rev. Lett. 107, 181801 (2011)

156. "Search for $\mathrm{B}(\mathrm{s})$ and $\mathrm{B}$ to dimuon decays in pp collisions at $7 \mathrm{TeV}$ "

S. Chatrchyan et al. [CMS Collaboration].

arXiv:1107.5834 [hep-ex]

Phys. Rev. Lett. 107, 191802 (2011)

157. "A Search for resonant production of $t \bar{t}$ pairs in $4.8 \mathrm{fb}^{-1}$ of integrated luminosity of $p \bar{p}$ collisions at $\sqrt{s}=1.96 \mathrm{TeV}$ "

T. Aaltonen et al. [CDF Collaboration].

arXiv:1107.5063 [hep-ex]

Phys. Rev. D 84, 072004 (2011)

158. "Measurement of Polarization and Search for CP-Violation in $B_{s}^{0} \rightarrow \phi \phi$ Decays" T. Aaltonen et al. [CDF Collaboration].

arXiv:1107.4999 [hep-ex]

Phys. Rev. Lett. 107, 261802 (2011) 
159. "Search for Resonances in the Dijet Mass Spectrum from 7 TeV pp Collisions at CMS"

S. Chatrchyan et al. [CMS Collaboration].

arXiv:1107.4771 [hep-ex]

Phys. Lett. B 704, 123 (2011)

160. "Measurement of the Inclusive $\mathbf{W}$ and $\mathrm{Z}$ Production Cross Sections in pp Collisions at $\operatorname{sqrt}(\mathrm{s})=7 \mathrm{TeV}$ "

S. Chatrchyan et al. [CMS Collaboration].

arXiv:1107.4789 [hep-ex]

JHEP 1110, 132 (2011)

161. "Dependence on pseudorapidity and centrality of charged hadron production in $\mathrm{PbPb}$ collisions at a nucleon-nucleon centre-of-mass energy of $2.76 \mathrm{TeV}$ "

S. Chatrchyan et al. [CMS Collaboration]. arXiv:1107.4800 [nucl-ex]

JHEP 1108, 141 (2011)

162. "Determination of Jet Energy Calibration and Transverse Momentum Resolution in CMS"

S. Chatrchyan et al. [CMS Collaboration].

arXiv:1107.4277 [physics.ins-det]

JINST 6, P11002 (2011)

163. "Search for a Heavy Top-Like Quark in $p \bar{p}$ Collisions at $\sqrt{ } s=1.96$ TeV"

T. Aaltonen et al. [CDF Collaboration].

arXiv:1107.3875 [hep-ex]

Phys. Rev. Lett. 107, 261801 (2011)

164. "Observation of the $\Xi_{b}^{0}$ Baryon"

T. Aaltonen et al. [CDF Collaboration].

arXiv:1107.4015 [hep-ex]

Phys. Rev. Lett. 107, 102001 (2011)

165. "Search for New $T^{\prime}$ Particles in Final States with Large Jet Multiplicities and Missing Transverse Energy in $p \bar{p}$ Collisions at sqrt(s) $=1.96 \mathrm{TeV}^{\prime \prime}$

T. Aaltonen et al. [CDF Collaboration].

arXiv:1107.3574 [hep-ex]

Phys. Rev. Lett. 107, 191803 (2011)

166. "Observation of the Baryonic Flavor-Changing Neutral Current Decay $\Lambda_{b} \rightarrow$ $\Lambda \mu^{+} \mu^{-}$

T. Aaltonen et al. [CDF Collaboration].

arXiv:1107.3753 [hep-ex]

Phys. Rev. Lett. 107, 201802 (2011)

167. "Search for Three-Jet Resonances in pp Collisions at sqrt(s) $=7 \mathrm{TeV}$ "

S. Chatrchyan et al. [CMS Collaboration]. 
arXiv:1107.3084 [hep-ex]

Phys. Rev. Lett. 107, 101801 (2011)

168. "Search for $B_{s} \rightarrow \mu^{+} \mu^{-}$and $B_{d} \rightarrow \mu^{+} \mu^{-}$Decays with CDF II"

T. Aaltonen et al. [CDF Collaboration].

arXiv:1107.2304 [hep-ex]

Phys. Rev. Lett. 107, 239903 (2011), [Phys. Rev. Lett. 107, 191801 (2011)]

169. "Search for supersymmetry in pp collisions at $\operatorname{sqrt}(\mathrm{s})=\mathbf{7} \mathrm{TeV}$ in events with a single lepton, jets, and missing transverse momentum"

S. Chatrchyan et al. [CMS Collaboration].

arXiv:1107.1870 [hep-ex]

JHEP 1108, 156 (2011)

170. "A search for excited leptons in pp Collisions at $\operatorname{sqrt}(\mathrm{s})=7 \mathrm{TeV}$ "

S. Chatrchyan et al. [CMS Collaboration].

arXiv:1107.1773 [hep-ex]

Phys. Lett. B 704, 143 (2011)

171. "Inclusive search for squarks and gluinos in pp collisions at $\operatorname{sqrt}(\mathrm{s})=7 \mathrm{TeV}$ "

S. Chatrchyan et al. [CMS Collaboration].

arXiv:1107.1279 [hep-ex]

Phys. Rev. D 85, 012004 (2012)

172. "Measurement of the Underlying Event Activity at the LHC with $\sqrt{s}=7$ TeV and Comparison with $\sqrt{s}=0.9$ TeV"

S. Chatrchyan et al. [CMS Collaboration].

arXiv:1107.0330 [hep-ex]

JHEP 1109, 109 (2011)

173. "Study of Substructure of High Transverse Momentum Jets Produced in ProtonAntiproton Collisions at $\sqrt{s}=1.96 \mathbf{T e V}^{\prime \prime}$

T. Aaltonen et al. [CDF Collaboration].

arXiv:1106.5952 [hep-ex]

Phys. Rev. D 85, 091101 (2012)

174. "Measurement of the Cross Section for Prompt Isolated Diphoton Production in $p \bar{p}$ Collisions at $\sqrt{s}=1.96 \mathbf{T e V}$ "

T. Aaltonen et al. [CDF Collaboration].

arXiv:1106.5123 [hep-ex]

Phys. Rev. Lett. 107, 102003 (2011)

175. "Measurement of the Cross Section for Prompt Isolated Diphoton Production in $p \bar{p}$ Collisions at $\sqrt{s}=1.96 \mathbf{T e V}$ "

T. Aaltonen et al. [CDF Collaboration].

arXiv:1106.5131 [hep-ex]

Phys. Rev. D 84, 052006 (2011) 
176. "Missing transverse energy performance of the CMS detector"

S. Chatrchyan et al. [CMS Collaboration].

arXiv:1106.5048 [physics.ins-det]

JINST 6, P09001 (2011)

177. "Search for New Physics with a Mono-Jet and Missing Transverse Energy in $p p$ Collisions at $\sqrt{s}=7 \mathrm{TeV}^{\prime \prime}$

S. Chatrchyan et al. [CMS Collaboration].

arXiv:1106.4775 [hep-ex]

Phys. Rev. Lett. 107, 201804 (2011)

178. "Search for Higgs Bosons Produced in Association with $b$-quarks"

T. Aaltonen et al. [CDF Collaboration].

arXiv:1106.4782 [hep-ex]

Phys. Rev. D 85, 032005 (2012)

179. "Search for New Physics with Jets and Missing Transverse Momentum in pp collisions at sqrt(s) $=7 \mathrm{TeV}$ "

S. Chatrchyan et al. [CMS Collaboration].

arXiv:1106.4503 [hep-ex]

JHEP 1108, 155 (2011)

180. "Measurement of the Strange B Meson Production Cross Section with J/Psi phi Decays in pp Collisions at sqrt(s) $=7$ TeV"

S. Chatrchyan et al. [CMS Collaboration].

arXiv:1106.4048 [hep-ex]

Phys. Rev. D 84, 052008 (2011)

181. "Evidence for $t \bar{t} \gamma$ Production and Measurement of $\sigma_{t} \bar{t} \gamma / \sigma_{t} \bar{t} "$

T. Aaltonen et al. [CDF Collaboration].

arXiv:1106.3970 [hep-ex]

Phys. Rev. D 84, 031104 (2011)

182. "Measurement of branching ratio and $B_{s}^{0}$ lifetime in the decay $B_{s}^{0} \rightarrow J / \psi f_{0}(980)$ at CDF"

T. Aaltonen et al. [CDF Collaboration].

arXiv:1106.3682 [hep-ex]

Phys. Rev. D 84, 052012 (2011)

183. "Search for Supersymmetry in Events with b Jets and Missing Transverse Momentum at the LHC"

S. Chatrchyan et al. [CMS Collaboration].

arXiv:1106.3272 [hep-ex]

JHEP 1107, 113 (2011)

184. "Measurement of the t-channel single top quark production cross section in pp collisions at $\operatorname{sqrt}(\mathrm{s})=7 \mathrm{TeV}$ "

S. Chatrchyan et al. [CMS Collaboration]. 
arXiv:1106.3052 [hep-ex]

Phys. Rev. Lett. 107, 091802 (2011)

185. "Search for Light Resonances Decaying into Pairs of Muons as a Signal of New Physics"

S. Chatrchyan et al. [CMS Collaboration].

arXiv:1106.2375 [hep-ex]

JHEP 1107, 098 (2011)

186. "Search for Same-Sign Top-Quark Pair Production at sqrt(s) $=7 \mathrm{TeV}$ and Limits on Flavour Changing Neutral Currents in the Top Sector"

S. Chatrchyan et al. [CMS Collaboration].

arXiv:1106.2142 [hep-ex]

JHEP 1108, 005 (2011)

187. "The T2K Experiment"

K. Abe et al. [T2K Collaboration].

arXiv:1106.1238 [physics.ins-det]

Nucl. Instrum. Meth. A 659, 106 (2011)

188. "Measurement of the Top-antitop Production Cross Section in $p p$ Collisions at $\sqrt{s}=7 \mathrm{TeV}$ using the Kinematic Properties of Events with Leptons and Jets"

S. Chatrchyan et al. [CMS Collaboration].

arXiv:1106.0902 [hep-ex]

Eur. Phys. J. C 71, 1721 (2011)

189. "Search for Physics Beyond the Standard Model Using Multilepton Signatures in $p p$ Collisions at $\sqrt{s}=7 \mathrm{TeV}$ "

S. Chatrchyan et al. [CMS Collaboration].

arXiv:1106.0933 [hep-ex]

Phys. Lett. B 704, 411 (2011)

190. "Measurement of the Ratio of the 3-jet to 2-jet Cross Sections in $p p$ Collisions at $\sqrt{s}=7 \mathrm{TeV} "$

S. Chatrchyan et al. [CMS Collaboration].

arXiv:1106.0647 [hep-ex]

Phys. Lett. B 702, 336 (2011)

191. "Neutrino Quasielastic Scattering on Nuclear Targets: Parametrizing Transverse Enhancement (Meson Exchange Currents)"

A. Bodek, H. S. Budd and M. E. Christy.

arXiv:1106.0340 [hep-ph]

Eur. Phys. J. C 71, 1726 (2011)

192. "Measurement of the Inclusive Jet Cross Section in pp Collisions at $\operatorname{sqrt}(\mathrm{s})=7$ TeV"

S. Chatrchyan et al. [CMS Collaboration]. 
arXiv:1106.0208 [hep-ex]

Phys. Rev. Lett. 107, 132001 (2011)

193. "Measurements of the properties of $\Lambda_{c}(2595), \Lambda_{c}(2625), \Sigma_{c}(2455)$, and $\Sigma_{c}(2520)$ baryons"

T. Aaltonen et al. [CDF Collaboration].

arXiv:1105.5995 [hep-ex]

Phys. Rev. D 84, 012003 (2011)

194. "Improved determination of the sample composition of dimuon events produced in $p \bar{p}$ collisions at $\sqrt{s}=1.96 \mathrm{TeV}$ "

T. Aaltonen et al. [CDF Collaboration].

arXiv:1105.5787 [hep-ex]

Eur. Phys. J. C 71, 1720 (2011)

195. "Measurement of the $\mathrm{t} t$-bar production cross section and the top quark mass in the dilepton channel in pp collisions at $\operatorname{sqrt}(\mathrm{s})=7 \mathrm{TeV}$ "

S. Chatrchyan et al. [CMS Collaboration].

arXiv:1105.5661 [hep-ex]

JHEP 1107, 049 (2011)

196. "Search for First Generation Scalar Leptoquarks in the evjj channel in pp collisions at $\operatorname{sqrt}(\mathrm{s})=7 \mathrm{TeV}$ "

S. Chatrchyan et al. [CMS Collaboration].

arXiv:1105.5237 [hep-ex]

Phys. Lett. B 703, 246 (2011)

197. "Indications of suppression of excited $\Upsilon$ states in $\mathrm{PbPb}$ collisions at $\sqrt{S_{N N}}=\mathbf{2 . 7 6}$ TeV"

S. Chatrchyan et al. [CMS Collaboration]. arXiv:1105.4894 [nucl-ex]

Phys. Rev. Lett. 107, 052302 (2011)

198. "Search for supersymmetry in events with a lepton, a photon, and large missing transverse energy in $p p$ collisions at $\sqrt{s}=7 \mathrm{TeV}$ "

S. Chatrchyan et al. [CMS Collaboration].

arXiv:1105.3152 [hep-ex]

JHEP 1106, 093 (2011)

199. "Measurement of $W \gamma$ and $Z \gamma$ production in $p p$ collisions at $\sqrt{s}=7 \mathrm{TeV}$ "

S. Chatrchyan et al. [CMS Collaboration].

arXiv:1105.2758 [hep-ex]

Phys. Lett. B 701, 535 (2011)

200. "First Search for Multijet Resonances in $\sqrt{s}=1.96 \mathrm{TeV} p \bar{p}$ Collisions"

T. Aaltonen et al. [CDF Collaboration].

arXiv:1105.2815 [hep-ex]

Phys. Rev. Lett. 107, 042001 (2011) 
201. "Long-range and short-range dihadron angular correlations in central $\mathbf{P b P b}$ collisions at a nucleon-nucleon center of mass energy of $2.76 \mathrm{TeV}$ "

S. Chatrchyan et al. [CMS Collaboration]. arXiv:1105.2438 [nucl-ex]

JHEP 1107, 076 (2011)

202. "Measurement of the $t \bar{t}$ production cross section in $p \bar{p}$ collisions at $\sqrt{s}=1.96 \mathrm{TeV}$ using events with large Missing $E_{T}$ and jets"

T. Aaltonen et al. [CDF Collaboration].

arXiv:1105.1806 [hep-ex]

Phys. Rev. D 84, 032003 (2011)

203. "Top quark mass measurement using the template method at CDF"

T. Aaltonen et al. [CDF Collaboration].

arXiv:1105.0192 [hep-ex]

Phys. Rev. D 83, 111101 (2011)

204. "Search for a Very Light CP-Odd Higgs Boson in Top Quark Decays from $\mathbf{p}^{-} p$ Collisions at $1.96 \mathrm{TeV}$ "

T. Aaltonen et al. [CDF Collaboration].

arXiv:1104.5701 [hep-ex]

Phys. Rev. Lett. 107, 031801 (2011)

205. "Charged particle transverse momentum spectra in $p p$ collisions at $\sqrt{s}=0.9$ and 7 TeV"

S. Chatrchyan et al. [CMS Collaboration].

arXiv:1104.3547 [hep-ex]

JHEP 1108, 086 (2011)

206. "Measurement of the Polarization of W Bosons with Large Transverse Momenta in W+Jets Events at the LHC"

S. Chatrchyan et al. [CMS Collaboration].

arXiv:1104.3829 [hep-ex]

Phys. Rev. Lett. 107, 021802 (2011)

207. "Search for new physics with same-sign isolated dilepton events with jets and missing transverse energy at the LHC"

S. Chatrchyan et al. [CMS Collaboration].

arXiv:1104.3168 [hep-ex]

JHEP 1106, 077 (2011)

208. "Measurement of the B0 production cross section in pp Collisions at sqrt(s) $=7$ TeV"

S. Chatrchyan et al. [CMS Collaboration].

arXiv:1104.2892 [hep-ex]

Phys. Rev. Lett. 106, 252001 (2011) 
209. "Measurement of the differential dijet production cross section in proton-proton collisions at $\sqrt{s}=7 \mathrm{TeV}^{\prime \prime}$

S. Chatrchyan et al. [CMS Collaboration].

arXiv:1104.1693 [hep-ex]

Phys. Lett. B 700, 187 (2011)

210. "Search for the Rare Radiative Decay: $W \rightarrow \pi \gamma$ in $p \bar{p}$ Collisions at $\sqrt{s}=1.96$ TeV" T. Aaltonen et al. [CDF Collaboration].

arXiv:1104.1585 [hep-ex]

Phys. Rev. D 85, 032001 (2012)

211. "Measurement of the Inclusive Z Cross Section via Decays to Tau Pairs in $p p$ Collisions at $\sqrt{s}=7 \mathrm{TeV}$ "

S. Chatrchyan et al. [CMS Collaboration].

arXiv:1104.1617 [hep-ex]

JHEP 1108, 117 (2011)

212. "Search for Neutral MSSM Higgs Bosons Decaying to Tau Pairs in $p p$ Collisions at $\sqrt{s}=7 \mathrm{TeV} "$

S. Chatrchyan et al. [CMS Collaboration].

arXiv:1104.1619 [hep-ex]

Phys. Rev. Lett. 106, 231801 (2011)

213. "Invariant Mass Distribution of Jet Pairs Produced in Association with a $W$ boson in $p \bar{p}$ Collisions at $\sqrt{s}=1.96 \mathbf{T e V}^{\prime}$

T. Aaltonen et al. [CDF Collaboration].

arXiv:1104.0699 [hep-ex]

Phys. Rev. Lett. 106, 171801 (2011)

214. "Measurements of Direct CP Violating Asymmetries in Charmless Decays of Strange Bottom Mesons and Bottom Baryons"

T. Aaltonen et al. [CDF Collaboration].

arXiv:1103.5762 [hep-ex]

Phys. Rev. Lett. 106, 181802 (2011)

215. "First Measurement of the Angular Coefficients of Drell-Yan $e^{+} e^{-}$pairs in the Z Mass Region from $p \bar{p}$ Collisions at $\sqrt{s}=1.96$ TeV"

T. Aaltonen et al. [CDF Collaboration].

arXiv:1103.5699 [hep-ex]

Phys. Rev. Lett. 106, 241801 (2011)

216. "Measurement of Event Shapes in Proton-Antiproton Collisions at Center-ofMass Energy 1.96 TeV"

T. Aaltonen et al. [CDF Collaboration].

arXiv:1103.5143 [hep-ex]

Phys. Rev. D 83, 112007 (2011) 
217. "Search for New Dielectron Resonances and Randall-Sundrum Gravitons at the Collider Detector at Fermilab"

T. Aaltonen et al. [CDF Collaboration].

arXiv:1103.4650 [hep-ex]

Phys. Rev. Lett. 107, 051801 (2011)

218. "Measurement of the Top Pair Production Cross Section in the Lepton + Jets Channel Using a Jet Flavor Discriminant"

T. Aaltonen et al. [CDF Collaboration].

arXiv:1103.4821 [hep-ex]

Phys. Rev. D 84, 031101 (2011)

219. "Search for Large Extra Dimensions in the Diphoton Final State at the Large Hadron Collider"

S. Chatrchyan et al. [CMS Collaboration].

arXiv:1103.4279 [hep-ex]

JHEP 1105, 085 (2011)

220. "Measurement of the lepton charge asymmetry in inclusive $W$ production in pp collisions at $\sqrt{s}=7 \mathrm{TeV}^{\prime \prime}$

S. Chatrchyan et al. [CMS Collaboration].

arXiv:1103.3470 [hep-ex]

JHEP 1104, 050 (2011)

221. "Combined CDF and Do Upper Limits on Standard Model Higgs Boson Production with up to $8.2 \mathrm{fb}^{-1}$ of Data"

T. Aaltonen et al. [CDF and D0 Collaboration].

arXiv:1103.3233 [hep-ex]

222. "Measurement of the mass difference between $t$ and $\bar{t}$ quarks"

T. Aaltonen et al. [CDF Collaboration].

arXiv:1103.2782 [hep-ex]

Phys. Rev. Lett. 106, 152001 (2011)

223. "Limits on Anomalous Trilinear Gauge Couplings in $Z \gamma$ Events from $p \bar{p}$ Collisions at $\sqrt{s}=1.96 \mathrm{TeV}^{\prime \prime}$

T. Aaltonen et al. [CDF Collaboration].

arXiv:1103.2990 [hep-ex]

Phys. Rev. Lett. 107, 051802 (2011)

224. "Search for Production of Heavy Particles Decaying to Top Quarks and Invisible Particles in $p \bar{p}$ collisions at $\sqrt{s}=1.96 \mathrm{TeV}$ "

T. Aaltonen et al. [CDF Collaboration].

arXiv:1103.2482 [hep-ex]

Phys. Rev. Lett. 106, 191801 (2011)

225. "Measurement of the $B_{s}$ Lifetime in Fully and Partially Reconstructed $B_{s} \rightarrow$ $D_{s}^{-}\left(\phi \pi^{-}\right) X$ Decays in $\bar{p}-p$ Collisions at $\sqrt{s}=1.96 \mathbf{T e V}^{\prime}$ 
T. Aaltonen et al. [CDF Collaboration].

arXiv:1103.1864 [hep-ex]

Phys. Rev. Lett. 107, 272001 (2011)

226. "Search for Physics Beyond the Standard Model in Opposite-Sign Dilepton Events at $\sqrt{s}=7 \mathrm{TeV}$ "

S. Chatrchyan et al. [CMS Collaboration].

arXiv:1103.1348 [hep-ex]

JHEP 1106, 026 (2011)

227. "Search for Supersymmetry in $p p$ Collisions at $\sqrt{s}=7 \mathrm{TeV}$ in Events with Two Photons and Missing Transverse Energy"

S. Chatrchyan et al. [CMS Collaboration].

arXiv:1103.0953 [hep-ex]

Phys. Rev. Lett. 106, 211802 (2011)

228. "Search for Resonances in the Dilepton Mass Distribution in $p p$ Collisions at $\sqrt{(s)}=7$ TeV"

S. Chatrchyan et al. [CMS Collaboration].

arXiv:1103.0981 [hep-ex]

JHEP 1105, 093 (2011)

229. "Search for a $W^{\prime}$ boson decaying to a muon and a neutrino in $p p$ collisions at $\sqrt{s}=7$ TeV"

S. Chatrchyan et al. [CMS Collaboration].

arXiv:1103.0030 [hep-ex]

Phys. Lett. B 701, 160 (2011)

230. "Measurement of $\mathbf{W}+\mathbf{W}$ - Production and Search for the Higgs Boson in pp Collisions at sqrt(s) $=7 \mathrm{TeV}$ "

S. Chatrchyan et al. [CMS Collaboration].

arXiv:1102.5429 [hep-ex]

Phys. Lett. B 699, 25 (2011)

231. "Study of $\mathrm{Z}$ boson production in $\mathrm{PbPb}$ collisions at nucleon-nucleon centre of mass energy $=2.76 \mathrm{TeV}$ "

S. Chatrchyan et al. [CMS Collaboration].

arXiv:1102.5435 [nucl-ex]

Phys. Rev. Lett. 106, 212301 (2011)

232. "Search for a Heavy Bottom-like Quark in $p p$ Collisions at $\sqrt{s}=7 \mathbf{T e V}$ "

S. Chatrchyan et al. [CMS Collaboration].

arXiv:1102.4746 [hep-ex]

Phys. Lett. B 701, 204 (2011)

233. "Search for New Heavy Particles Decaying to $Z Z \rightarrow \ell \ell \ell \ell, \ell \ell j j$ in $p \bar{p}$ Collisions at $\sqrt{s}=1.96$ TeV"

T. Aaltonen et al. [CDF Collaboration]. 
arXiv:1102.4566 [hep-ex]

Phys. Rev. D 83, 112008 (2011)

234. "Strange Particle Production in pp Collisions at sqrt(s) $=0.9$ and $7 \mathrm{TeV}$ "

V. Khachatryan et al. [CMS Collaboration].

arXiv:1102.4282 [hep-ex]

JHEP 1105, 064 (2011)

235. "Measurement of $B \bar{B}$ Angular Correlations based on Secondary Vertex Reconstruction at $\sqrt{s}=7 \mathbf{T e V}$ "

V. Khachatryan et al. [CMS Collaboration].

arXiv:1102.3194 [hep-ex]

JHEP 1103, 136 (2011)

236. "Measurement of Dijet Angular Distributions and Search for Quark Compositeness in pp Collisions at sqrts $=7 \mathrm{TeV}^{\prime}$

V. Khachatryan et al. [CMS Collaboration].

arXiv:1102.2020 [hep-ex]

Phys. Rev. Lett. 106, 201804 (2011)

237. "Observation and studies of jet quenching in $\mathrm{PbPb}$ collisions at nucleon-nucleon center-of-mass energy $=2.76 \mathrm{TeV}$ "

S. Chatrchyan et al. [CMS Collaboration].

arXiv:1102.1957 [nucl-ex]

Phys. Rev. C 84, 024906 (2011)

238. "Observation of $B_{s}^{0} \rightarrow J / \psi K^{* 0}(892)$ and $B_{s}^{0} \rightarrow J / \psi K_{S}^{0}$ Decays"

T. Aaltonen et al. [CDF Collaboration].

arXiv:1102.1961 [hep-ex]

Phys. Rev. D 83, 052012 (2011)

239. "Observation of the $Y(4140)$ structure in the $J / \psi \phi$ Mass Spectrum in $B^{ \pm} \rightarrow J / \psi \phi K$ decays"

T. Aaltonen et al. [CDF Collaboration].

arXiv:1101.6058 [hep-ex]

240. "Search for the Higgs boson in the all-hadronic final state using the CDF II detector"

T. Aaltonen et al. [CDF Collaboration].

arXiv:1102.0024 [hep-ex]

Phys. Rev. D 84, 052010 (2011)

241. "First Measurement of Hadronic Event Shapes in $p p$ Collisions at $\sqrt{(s)}=7$ TeV" V. Khachatryan et al. [CMS Collaboration].

arXiv:1102.0068 [hep-ex]

Phys. Lett. B 699, 48 (2011) 
242. "Search for heavy bottom-like quarks decaying to an electron or muon and jets in $p \bar{p}$ collisions at $\sqrt{s}=1.96 \mathbf{T e V}$ "

T. Aaltonen et al. [CDF Collaboration].

arXiv:1101.5728 [hep-ex]

Phys. Rev. Lett. 106, 141803 (2011)

243. "Dijet Azimuthal Decorrelations in $p p$ Collisions at $\sqrt{s}=7$ TeV"

V. Khachatryan et al. [CMS Collaboration].

arXiv:1101.5029 [hep-ex]

Phys. Rev. Lett. 106, 122003 (2011)

244. "Measurement of the Top Quark Mass in the Lepton+Jets Channel Using the Lepton Transverse Momentum"

T. Aaltonen et al. [CDF Collaboration].

arXiv:1101.4926 [hep-ex]

Phys. Lett. B 698, 371 (2011)

245. "Search for High Mass Resonances Decaying to Muon Pairs in $\sqrt{s}=1.96 \mathrm{TeV} p \bar{p}$ Collisions"

T. Aaltonen et al. [CDF Collaboration].

arXiv:1101.4578 [hep-ex]

Phys. Rev. Lett. 106, 121801 (2011)

246. "Inclusive b-hadron production cross section with muons in $p p$ collisions at $\sqrt{s}=7$ TeV"

V. Khachatryan et al. [CMS Collaboration].

arXiv:1101.3512 [hep-ex]

JHEP 1103, 090 (2011)

247. "Measurement of Bose-Einstein Correlations in $p p$ Collisions at $\sqrt{s}=0.9$ and 7 TeV"

V. Khachatryan et al. [CMS Collaboration].

arXiv:1101.3518 [hep-ex]

JHEP 1105, 029 (2011)

248. "Production of $\Lambda, \bar{\Lambda}^{0} \Xi^{ \pm}$and $\Omega^{ \pm}$Hyperons in $p \bar{p}$ Collisions at $\sqrt{s}=1.96 \mathbf{T e V}$ "

T. Aaltonen et al. [CDF Collaboration].

arXiv:1101.2996 [hep-ex]

Phys. Rev. D 86, 012002 (2012)

249. "Search for Supersymmetry in pp Collisions at $7 \mathrm{TeV}$ in Events with Jets and Missing Transverse Energy"

V. Khachatryan et al. [CMS Collaboration].

arXiv:1101.1628 [hep-ex]

Phys. Lett. B 698, 196 (2011)

250. "Search for Heavy Stable Charged Particles in pp collisions at sqrt(s)=7 TeV" V. Khachatryan et al. [CMS Collaboration]. 
arXiv:1101.1645 [hep-ex]

JHEP 1103, 024 (2011)

251. "Measurement of the Forward-Backward Asymmetry in the $B \rightarrow K^{(*)} \mu^{+} \mu^{-}$Decay and First Observation of the $B_{s}^{0} \rightarrow \phi \mu^{+} \mu^{-}$Decay"

T. Aaltonen et al. [CDF Collaboration].

arXiv:1101.1028 [hep-ex]

Phys. Rev. Lett. 106, 161801 (2011)

252. "Measurement of the $B^{+}$Production Cross Section in pp Collisions at $\sqrt{s}=$ $7 \mathrm{TeV} "$

V. Khachatryan et al. [CMS Collaboration].

arXiv:1101.0131 [hep-ex]

Phys. Rev. Lett. 106, 112001 (2011)

253. "Evidence for a Mass Dependent Forward-Backward Asymmetry in Top Quark Pair Production"

T. Aaltonen et al. [CDF Collaboration].

arXiv:1101.0034 [hep-ex]

Phys. Rev. D 83, 112003 (2011)

254. "Search for a heavy gauge boson $\mathrm{W}$ ' in the final state with an electron and large missing transverse energy in pp collisions at $\operatorname{sqrt}(\mathrm{s})=7 \mathrm{TeV}$ "

V. Khachatryan et al. [CMS Collaboration].

arXiv:1012.5945 [hep-ex]

Phys. Lett. B 698, 21 (2011)

255. "Measurement of the Inclusive Upsilon production cross section in pp collisions at $\operatorname{sqrt}(\mathrm{s})=7 \mathrm{TeV} "$

V. Khachatryan et al. [CMS Collaboration].

arXiv:1012.5545 [hep-ex]

Phys. Rev. D 83, 112004 (2011)

256. "Search for a New Heavy Gauge Boson $W^{\prime}$ with Electron + missing ET Event Signature in $p \bar{p}$ collisions at $\sqrt{s}=1.96 \mathrm{TeV}$ "

T. Aaltonen et al. [CDF Collaboration].

arXiv:1012.5145 [hep-ex]

Phys. Rev. D 83, 031102 (2011)

257. "Search for Pair Production of First-Generation Scalar Leptoquarks in pp Collisions at $\operatorname{sqrt}(\mathrm{s})=7 \mathrm{TeV}$ "

V. Khachatryan et al. [CMS Collaboration].

arXiv:1012.4031 [hep-ex]

Phys. Rev. Lett. 106, 201802 (2011)

258. "Search for Pair Production of Second-Generation Scalar Leptoquarks in pp Collisions at $\operatorname{sqrt}(\mathrm{s})=7 \mathrm{TeV}$ "

V. Khachatryan et al. [CMS Collaboration]. 
arXiv:1012.4033 [hep-ex]

Phys. Rev. Lett. 106, 201803 (2011)

259. "Search for Microscopic Black Hole Signatures at the Large Hadron Collider" V. Khachatryan et al. [CMS Collaboration].

arXiv:1012.3375 [hep-ex]

Phys. Lett. B 697, 434 (2011)

260. "Measurement of $t \bar{t}$ Spin Correlation in $p \bar{p}$ Collisions Using the CDF II Detector at the Tevatron"

T. Aaltonen et al. [CDF Collaboration].

arXiv:1012.3093 [hep-ex]

Phys. Rev. D 83, 031104 (2011)

261. "Measurement of $\mathbf{b}$ hadron lifetimes in exclusive decays containing a $\mathbf{J} / \mathbf{p s i}$ in p-pbar collisions at $\operatorname{sqrt}(\mathrm{s})=1.96 \mathrm{TeV}$ "

T. Aaltonen et al. [CDF Collaboration].

arXiv:1012.3138 [hep-ex]

Phys. Rev. Lett. 106, 121804 (2011)

262. "Measurements of Inclusive $\mathrm{W}$ and $\mathrm{Z}$ Cross Sections in pp Collisions at sqrt(s)=7 TeV"

V. Khachatryan et al. [CMS Collaboration].

arXiv:1012.2466 [hep-ex]

JHEP 1101, 080 (2011)

263. "Search for Randall-Sundrum Gravitons in the Diphoton Channel at CDF"

T. Aaltonen et al. [CDF Collaboration].

arXiv:1012.2795 [hep-ex]

Phys. Rev. D 83, 011102 (2011)

264. "Measurement of the Isolated Prompt Photon Production Cross Section in $p p$ Collisions at $\sqrt{s}=7$ TeV"

V. Khachatryan et al. [CMS Collaboration].

arXiv:1012.0799 [hep-ex]

Phys. Rev. Lett. 106, 082001 (2011)

265. "Charged particle multiplicities in pp interactions at $\operatorname{sqrt}(\mathrm{s})=\mathbf{0 . 9}, \mathbf{2 . 3 6}$, and 7 TeV"

V. Khachatryan et al. [CMS Collaboration].

arXiv:1011.5531 [hep-ex]

JHEP 1101, 079 (2011)

266. "Search for Stopped Gluinos in $p p$ collisions at $\sqrt{s}=7 \mathbf{T e V}$ "

V. Khachatryan et al. [CMS Collaboration].

arXiv:1011.5861 [hep-ex]

Phys. Rev. Lett. 106, 011801 (2011) 
267. "Update to the Bodek-Yang Unified Model for Electron- and Neutrino- Nucleon Scattering Cross Sections"

A. Bodek and U. -K. Yang.

arXiv:1012.0261 [hep-ph]

PoS ICHEP 2010, 292 (2010)

268. "Axial and Vector Structure Functions for Electron- and Neutrino- Nucleon Scattering Cross Sections at all $Q^{2}$ using Effective Leading order Parton Distribution Functions"

A. Bodek and U. -k. Yang.

arXiv:1011.6592 [hep-ph]

269. "Prompt and non-prompt $\mathbf{J} /$ psi production in pp collisions at $\operatorname{sqrt}(\mathrm{s})=7 \mathrm{TeV}$ " V. Khachatryan et al. [CMS Collaboration].

arXiv:1011.4193 [hep-ex]

Eur. Phys. J. C 71, 1575 (2011)

270. "Design, Performance, and Calibration of CMS Hadron-Barrel Calorimeter Wedges" G. Baiatian et al. [CMS HCAL Collaboration].

271. "Energy Response and Longitudinal Shower Profiles Measured in CMS HCAL and Comparison With Geant4"

G. Baiatian et al. [CMS HCAL Collaboration].

272. "Study of Various Photomultiplier Tubes with Muon Beams And Cerenkov Light Produced in Electron Showers"

S. Chatrchyan et al. [CMS HCAL Collaboration].

JINST 5, P06002 (2010).

273. "First Measurement of the Cross Section for Top-Quark Pair Production in Proton-Proton Collisions at sqrt(s)=7 TeV"

V. Khachatryan et al. [CMS Collaboration]. arXiv:1010.5994 [hep-ex]

Phys. Lett. B 695, 424 (2011)

274. "Top Quark Mass Measurement in the Lepton + Jets Channel Using a Matrix Element Method and in situ Jet Energy Calibration"

T. Aaltonen et al. [CDF Collaboration].

arXiv:1010.4582 [hep-ex]

Phys. Rev. Lett. 105, 252001 (2010)

275. "Search for Quark Compositeness with the Dijet Centrality Ratio in pp Collisions at $\operatorname{sqrt}(\mathrm{s})=7 \mathrm{TeV}$ "

V. Khachatryan et al. [CMS Collaboration].

arXiv:1010.4439 [hep-ex]

Phys. Rev. Lett. 105, 262001 (2010) 
276. "Search for Dijet Resonances in $7 \mathrm{TeV}$ pp Collisions at CMS"

V. Khachatryan et al. [CMS Collaboration].

arXiv:1010.0203 [hep-ex]

Phys. Rev. Lett. 105, 211801 (2010)

277. "Observation of Long-Range Near-Side Angular Correlations in Proton-Proton Collisions at the LHC"

V. Khachatryan et al. [CMS Collaboration].

arXiv:1009.4122 [hep-ex]

JHEP 1009, 091 (2010)

278. "Improved Search for a Higgs Boson Produced in Association with Z- $\mathrm{i} l+1-$ in proton antiproton Collisions at $\operatorname{sqrt}(\mathrm{s})=1.96 \mathrm{TeV}^{\prime \prime}$

T. Aaltonen et al. [CDF Collaboration].

arXiv:1009.3047 [hep-ex]

Phys. Rev. Lett. 105, 251802 (2010)

279. "Search for the supersymmetric partner of the top quark in $p \bar{p}$ collisions at sqrt(s)

$=1.96 \mathrm{TeV}$ "

T. Aaltonen et al. [CDF Collaboration].

arXiv:1009.0266 [hep-ex]

Phys. Rev. D 82, 092001 (2010)

280. "Updated Search for the Flavor-Changing Neutral-Current Decay $D^{0} \rightarrow \mu^{+} \mu^{-}$" T. Aaltonen et al. [CDF Collaboration].

arXiv:1008.5077 [hep-ex]

Phys. Rev. D 82, 091105 (2010)

281. "Measurement of the $W W+W Z$ Production Cross Section Using a Matrix Element Technique in Lepton + Jets Events"

T. Aaltonen et al. [CDF Collaboration].

arXiv:1008.4404 [hep-ex]

Phys. Rev. D 82, 112001 (2010)

282. "Direct Top-Quark Width Measurement CDF"

T. Aaltonen et al. [CDF Collaboration].

arXiv:1008.3891 [hep-ex]

Phys. Rev. Lett. 105, 232003 (2010)

283. "Diffractive W and Z Production at the Fermilab Tevatron"

T. Aaltonen et al. [CDF Collaboration].

arXiv:1007.5048 [hep-ex]

Phys. Rev. D 82, 112004 (2010)

284. "Measurement of the $t \bar{t}$ Production Cross Section with an in situ Calibration of $b$-jet Identification Efficiency"

T. Aaltonen et al. [CDF Collaboration]. 
arXiv:1007.4423 [hep-ex]

Phys. Rev. D 83, 071102 (2011)

285. "Study of various photomultiplier tubes with muon beams and Cherenkov light produced in electron showers"

S. Chatrchyan et al. [CMS HCAL Collaboration].

JINST 5, P06002 (2010).

286. "CMS Tracking Performance Results from early LHC Operation"

V. Khachatryan et al. [CMS Collaboration].

arXiv:1007.1988 [physics.ins-det]

Eur. Phys. J. C 70, 1165 (2010)

287. "Exclusion of an Exotic Top Quark with -4/3 Electric Charge Using Soft Lepton Tagging"

T. Aaltonen et al. [CDF Collaboration].

arXiv:1006.4597 [hep-ex]

Phys. Rev. Lett. 105, 101801 (2010)

288. "Dimuon production at NuTeV"

M. Goncharov, T. Adams, A. Alton, S. Avvakumov, L. de Barbaro, P. de Barbaro, R. H. Bernstein and A. Bodek et al..

289. "NUFACT09 update to the Bodek-Yang unified model for electron- and neutrinonucleon scattering cross sections"

A. Bodek and U. -k. Yang.

AIP Conf. Proc. 1222, 233 (2010).

290. "First Measurement of the Underlying Event Activity at the LHC with $\sqrt{s}=\mathbf{0 . 9}$ TeV"

V. Khachatryan et al. [CMS Collaboration].

arXiv:1006.2083 [hep-ex]

Eur. Phys. J. C 70, 555 (2010)

291. "Measurement of the charge ratio of atmospheric muons with the CMS detector"

V. Khachatryan et al. [CMS Collaboration].

arXiv:1005.5332 [hep-ex]

Phys. Lett. B 692, 83 (2010)

292. "Measurement of $d \sigma / d y$ of Drell-Yan $e^{+} e^{-}$pairs in the $Z$ Mass Region from $p \bar{p}$ Collisions at $\sqrt{s}=1.96 \mathrm{TeV}$ "

T. A. Aaltonen et al. [CDF Collaboration].

arXiv:0908.3914 [hep-ex], arXiv:0908.3914 [hep-ex]

Phys. Lett. B 692, 232 (2010)

293. "Combined Tevatron upper limit on $g g \rightarrow H \rightarrow W^{+} W^{-}$and constraints on the Higgs boson mass in fourth-generation fermion models"

T. Aaltonen et al. [CDF and D0 Collaboration]. 
arXiv:1005.3216 [hep-ex]

Phys. Rev. D 82, 011102 (2010)

294. "Measurement of Bose-Einstein correlations with first CMS data"

V. Khachatryan et al. [CMS Collaboration].

arXiv:1005.3294 [hep-ex]

Phys. Rev. Lett. 105, 032001 (2010)

295. "Transverse-momentum and pseudorapidity distributions of charged hadrons in pp collisions at sqrt(s) $=7 \mathrm{TeV}^{\prime \prime}$

V. Khachatryan et al. [CMS Collaboration].

arXiv:1005.3299 [hep-ex]

Phys. Rev. Lett. 105, 022002 (2010)

296. "Search for the Production of Scalar Bottom Quarks in $p \bar{p}$ collisions at $\sqrt{s}=1.96$ TeV"

T. Aaltonen et al. [CDF Collaboration].

arXiv:1005.3600 [hep-ex]

Phys. Rev. Lett. 105, 081802 (2010)

297. "Search for $W W$ and $W Z$ resonances decaying to electron, missing $E_{T}$, and two jets in $p \bar{p}$ collisions at $\sqrt{s}=1.96 \mathrm{TeV}^{\prime \prime}$

T. Aaltonen et al. [CDF Collaboration].

arXiv:1004.4946 [hep-ex]

Phys. Rev. Lett. 104, 241801 (2010)

298. "Measurement of the $B^{-}$lifetime using a simulation free approach for trigger bias correction"

T. Aaltonen et al. [CDF Collaboration].

arXiv:1004.4855 [hep-ex]

Phys. Rev. D 83, 032008 (2011)

299. "Search for R-parity Violating Decays of $\tau$ sneutrinos to $e \mu, \mu \tau$, and $e \tau$ Pairs in $p \bar{p}$ Collisions at $\sqrt{s}=1.96 \mathbf{T e V}^{\prime \prime}$

T. Aaltonen et al. [CDF Collaboration].

arXiv:1004.3042 [hep-ex]

Phys. Rev. Lett. 105, 191801 (2010)

300. "First Measurement of the Ratio $\sigma(t \bar{t}) / \sigma(Z / \gamma * * \rightarrow \ell \ell)$ and Precise Extraction of the $t \bar{t}$ Cross Section"

T. Aaltonen et al. [CDF Collaboration].

arXiv:1004.3224 [hep-ex]

Phys. Rev. Lett. 105, 012001 (2010)

301. "Observation of Single Top Quark Production and Measurement of - Vtb- with CDF"

T. Aaltonen et al. [CDF Collaboration]. 
arXiv:1004.1181 [hep-ex]

Phys. Rev. D 82, 112005 (2010)

302. "Measurement of $\mathbf{Z}$ gamma Production in $p \bar{p}$ Collisions at $\sqrt{s}=1.96$ TeV"

T. Aaltonen et al. [CDF Collaboration].

arXiv:1004.1140 [hep-ex]

Phys. Rev. D 82, 031103 (2010)

303. "Studying the Underlying Event in Drell-Yan and High Transverse Momentum Jet Production at the Tevatron"

T. Aaltonen et al. [CDF Collaboration].

arXiv:1003.3146 [hep-ex]

Phys. Rev. D 82, 034001 (2010)

304. "Measurement of W-Boson Polarization in Top-quark Decay in $p \bar{p}$ Collisions at $\operatorname{sqrt}(\mathrm{s})=1.96 \mathrm{TeV}^{\prime \prime}$

T. Aaltonen et al. [CDF Collaboration].

arXiv:1003.0224 [hep-ex]

Phys. Rev. Lett. 105, 042002 (2010)

305. "Measurement of the $t \bar{t}$ Production Cross Section in $p \bar{p}$ Collisions at sqrt(s)=1.96 TeV using Soft Electron b-Tagging"

T. Aaltonen et al. [CDF Collaboration].

arXiv:1002.3783 [hep-ex]

Phys. Rev. D 81, 092002 (2010)

306. "Measurement of the Top Pair Production Cross Section in the Dilepton Decay Channel in $p \bar{p}$ Collisions at sqrt $\mathrm{s}=1.96 \mathrm{TeV}$ "

T. Aaltonen et al. [CDF Collaboration].

arXiv:1002.2919 [hep-ex]

Phys. Rev. D 82, 052002 (2010)

307. "Transverse momentum and pseudorapidity distributions of charged hadrons in pp collisions at $\sqrt{(s)}=0.9$ and $2.36 \mathrm{TeV}^{\prime \prime}$

V. Khachatryan et al. [CMS Collaboration].

arXiv:1002.0621 [hep-ex]

JHEP 1002, 041 (2010)

308. "Measurement of the Top Quark Mass and $p \bar{p}-i t \bar{t}$ Cross Section in the AllHadronic Mode with the CDFII Detector"

T. Aaltonen et al. [CDF Collaboration].

arXiv:1002.0365 [hep-ex]

Phys. Rev. D 81, 052011 (2010)

309. "Combination of Tevatron searches for the standard model Higgs boson in the $\mathrm{W}+\mathrm{W}$ - decay mode"

T. Aaltonen et al. [CDF and D0 Collaboration]. 
arXiv:1001.4162 [hep-ex]

Phys. Rev. Lett. 104, 061802 (2010)

310. "Inclusive Search for Standard Model Higgs Boson Production in the WW Decay Channel using the CDF II Detector"

T. Aaltonen et al. [CDF Collaboration].

arXiv:1001.4468 [hep-ex]

Phys. Rev. Lett. 104, 061803 (2010)

311. "Search for single top quark production in pbar p collisions at sqrts=1.96 $\mathrm{TeV}$ in the missing transverse energy plus jets topology"

T. Aaltonen et al. [CDF Collaboration].

arXiv:1001.4577 [hep-ex]

Phys. Rev. D 81, 072003 (2010)

312. "Search for New Physics with a Dijet plus Missing Transverse Energy Signature in $p \bar{p}$ Collisions at $\sqrt{s}=1.96 \mathrm{TeV}$ "

T. Aaltonen et al. [CDF Collaboration].

arXiv:0912.4691 [hep-ex]

Phys. Rev. Lett. 105, 131801 (2010)

313. "Measurement of the W+ W- Production Cross Section and Search for Anomalous WW gamma and WWZ Couplings in p p-bar Collisions at $\mathrm{s}^{* *}(1 / 2)=1.96$ TeV"

T. Aaltonen et al. [CDF Collaboration].

arXiv:0912.4500 [hep-ex]

Phys. Rev. Lett. 104, 201801 (2010)

314. "Measurement of the Lambda0(b) Lifetime in Lambda0(b) - i Lambda+(c) piDecays in p p-bar Collisions at $\mathrm{s}^{* *}(1 / 2)=1.96-\mathrm{TeV}^{\prime \prime}$

T. Aaltonen et al. [CDF Collaboration].

arXiv:0912.3566 [hep-ex]

Phys. Rev. Lett. 104, 102002 (2010)

315. "A Study of the associated production of photons and b-quark jets in $p$ p-bar collisions at $\mathrm{s}^{* *}(1 / 2)=1.96-\mathrm{TeV} "$

T. Aaltonen et al. [CDF Collaboration].

arXiv:0912.3453 [hep-ex]

Phys. Rev. D 81, 052006 (2010)

316. "Search for Technicolor Particles Produced in Association with a W Boson at CDF"

T. Aaltonen et al. [CDF Collaboration].

arXiv:0912.2059 [hep-ex]

Phys. Rev. Lett. 104, 111802 (2010)

317. "Search for Pair Production of Supersymmetric Top Quarks in Dilepton Events from $p$ anti-p Collisions at $S^{* *}(1 / 2)=1.96 \mathrm{TeV}^{\prime \prime}$ 
T. Aaltonen et al. [CDF Collaboration].

arXiv:0912.1308 [hep-ex]

Phys. Rev. Lett. 104, 251801 (2010)

318. "Search for New Bottomlike Quark Pair Decays Q Q-bar - i (t W-+) (t-bar W+-) in Same-Charge Dilepton Events"

T. Aaltonen et al. [CDF Collaboration].

arXiv:0912.1057 [hep-ex]

Phys. Rev. Lett. 104, 091801 (2010)

319. "Commissioning and Performance of the CMS Pixel Tracker with Cosmic Ray Muons"

S. Chatrchyan et al. [CMS Collaboration].

arXiv:0911.5434 [physics.ins-det]

JINST 5, T03007 (2010)

320. "Performance of the CMS Level-1 Trigger during Commissioning with Cosmic Ray Muons"

S. Chatrchyan et al. [CMS Collaboration].

arXiv:0911.5422 [physics.ins-det]

JINST 5, T03002 (2010)

321. "Measurement of the Muon Stopping Power in Lead Tungstate"

S. Chatrchyan et al. [CMS Collaboration].

arXiv:0911.5397 [physics.ins-det]

JINST 5, P03007 (2010)

322. "Commissioning and Performance of the CMS Silicon Strip Tracker with Cosmic Ray Muons"

S. Chatrchyan et al. [CMS Collaboration].

arXiv:0911.4996 [physics.ins-det]

JINST 5, T03008 (2010)

323. "Performance of CMS Muon Reconstruction in Cosmic-Ray Events"

S. Chatrchyan et al. [CMS Collaboration].

arXiv:0911.4994 [physics.ins-det]

JINST 5, T03022 (2010)

324. "Performance of the CMS Cathode Strip Chambers with Cosmic Rays"

S. Chatrchyan et al. [CMS Collaboration].

arXiv:0911.4992 [physics.ins-det]

JINST 5, T03018 (2010)

325. "Performance of the CMS Hadron Calorimeter with Cosmic Ray Muons and LHC Beam Data"

S. Chatrchyan et al. [CMS Collaboration].

arXiv:0911.4991 [physics.ins-det]

JINST 5, T03012 (2010) 
326. "Fine Synchronization of the CMS Muon Drift-Tube Local Trigger using Cosmic Rays"

S. Chatrchyan et al. [CMS Collaboration].

arXiv:0911.4904 [physics.ins-det]

JINST 5, T03004 (2010)

327. "Calibration of the CMS Drift Tube Chambers and Measurement of the Drift Velocity with Cosmic Rays"

S. Chatrchyan et al. [CMS Collaboration].

arXiv:0911.4895 [physics.ins-det]

JINST 5, T03016 (2010)

328. "Performance of the CMS Drift-Tube Local Trigger with Cosmic Rays"

S. Chatrchyan et al. [CMS Collaboration].

arXiv:0911.4893 [physics.ins-det]

JINST 5, T03003 (2010)

329. "Commissioning of the CMS High-Level Trigger with Cosmic Rays"

S. Chatrchyan et al. [CMS Collaboration].

arXiv:0911.4889 [physics.ins-det]

JINST 5, T03005 (2010)

330. "Identification and Filtering of Uncharacteristic Noise in the CMS Hadron Calorimeter"

S. Chatrchyan et al. [CMS Collaboration].

arXiv:0911.4881 [physics.ins-det]

JINST 5, T03014 (2010)

331. "Performance of CMS Hadron Calorimeter Timing and Synchronization using Test Beam, Cosmic Ray, and LHC Beam Data"

S. Chatrchyan et al. [CMS Collaboration]. arXiv:0911.4877 [physics.ins-det]

JINST 5, T03013 (2010)

332. "Performance of the CMS Drift Tube Chambers with Cosmic Rays"

S. Chatrchyan et al. [CMS Collaboration].

arXiv:0911.4855 [physics.ins-det]

JINST 5, T03015 (2010)

333. "Commissioning of the CMS Experiment and the Cosmic Run at Four Tesla"

S. Chatrchyan et al. [CMS Collaboration].

arXiv:0911.4845 [physics.ins-det]

JINST 5, T03001 (2010)

334. "CMS Data Processing Workflows during an Extended Cosmic Ray Run"

S. Chatrchyan et al. [CMS Collaboration].

arXiv:0911.4842 [physics.ins-det]

JINST 5, T03006 (2010) 
335. "Aligning the CMS Muon Chambers with the Muon Alignment System during an Extended Cosmic Ray Run"

S. Chatrchyan et al. [CMS Collaboration].

arXiv:0911.4770 [physics.ins-det]

JINST 5, T03019 (2010)

336. "Measurement of the WW+WZ Production Cross Section Using the Lepton+Jets Final State at CDF II"

T. Aaltonen et al. [CDF Collaboration].

arXiv:0911.4449 [hep-ex]

Phys. Rev. Lett. 104, 101801 (2010)

337. "Performance Study of the CMS Barrel Resistive Plate Chambers with Cosmic Rays"

S. Chatrchyan et al. [CMS Collaboration].

arXiv:0911.4045 [physics.ins-det]

JINST 5, T03017 (2010)

338. "Time Reconstruction and Performance of the CMS Electromagnetic Calorimeter"

S. Chatrchyan et al. [CMS Collaboration].

arXiv:0911.4044 [physics.ins-det]

JINST 5, T03011 (2010)

339. "Alignment of the CMS Muon System with Cosmic-Ray and Beam-Halo Muons" S. Chatrchyan et al. [CMS Collaboration].

arXiv:0911.4022 [physics.ins-det]

JINST 5, T03020 (2010)

340. "A Search for the Higgs Boson Using Neural Networks in Events with Missing Energy and b-quark Jets in $p$ anti-p Collisions at $s^{* *}(1 / 2)=1.96-\mathrm{TeV}^{\prime \prime}$

T. Aaltonen et al. [CDF Collaboration].

arXiv:0911.3935 [hep-ex]

Phys. Rev. Lett. 104, 141801 (2010)

341. "Search for New Color-Octet Vector Particle Decaying to t anti-t in p anti-p Collisions at $\mathrm{s}^{* *}(1 / 2)=1.96-\mathrm{TeV}$

T. Aaltonen et al. [CDF Collaboration].

arXiv:0911.3112 [hep-ex]

Phys. Lett. B 691, 183 (2010)

342. "Top Quark Mass Measurement using mT2 in the Dilepton Channel at CDF" T. Aaltonen et al. [CDF Collaboration].

arXiv:0911.2956 [hep-ex]

Phys. Rev. D 81, 031102 (2010)

343. "A simple event weighting technique for optimizing the measurement of the forward-backward asymmetry of Drell-Yan dilepton pairs at hadron colliders" 
A. Bodek.

arXiv:0911.2850 [hep-ex]

Eur. Phys. J. C 67, 321 (2010)

344. "Measurements of branching fraction ratios and $\mathbf{C P}$ asymmetries in $\mathbf{B}+-$ - $_{\text {i }}$ $\mathrm{D}(\mathrm{CP}) \mathrm{K}+-$ decays in hadron collisions"

T. Aaltonen et al. [CDF Collaboration].

arXiv:0911.0425 [hep-ex]

Phys. Rev. D 81, 031105 (2010)

345. "Precise Mapping of the Magnetic Field in the CMS Barrel Yoke using Cosmic Rays"

S. Chatrchyan et al. [CMS Collaboration].

arXiv:0910.5530 [physics.ins-det]

JINST 5, T03021 (2010)

346. "Search for Anomalous Production of Events with Two Photons and Additional Energetic Objects at CDF"

T. Aaltonen et al. [CDF Collaboration].

arXiv:0910.5170 [hep-ex]

Phys. Rev. D 82, 052005 (2010)

347. "Measurement of the Inclusive Isolated Prompt Photon Cross Section in p anti-p Collisions at $\mathrm{s}^{* *}(1 / 2)=1.96-\mathrm{TeV}$ using the CDF Detector"

T. Aaltonen et al. [CDF Collaboration].

arXiv:0910.3623 [hep-ex]

Phys. Rev. D 80, 111106 (2009)

348. "Search for Supersymmetry with Gauge-Mediated Breaking in Diphoton Events with Missing Transverse Energy at CDF II"

T. Aaltonen et al. [CDF Collaboration].

arXiv:0910.3606 [hep-ex]

Phys. Rev. Lett. 104, 011801 (2010)

349. "Performance and Operation of the CMS Electromagnetic Calorimeter"

S. Chatrchyan et al. [CMS Collaboration].

arXiv:0910.3423 [physics.ins-det]

JINST 5, T03010 (2010)

350. "Alignment of the CMS Silicon Tracker during Commissioning with Cosmic Rays"

S. Chatrchyan et al. [CMS Collaboration].

arXiv:0910.2505 [physics.ins-det]

JINST 5, T03009 (2010)

351. "Measurements of the top-quark mass using charged particle tracking"

T. Aaltonen et al. [CDF Collaboration]. 
arXiv:0910.0969 [hep-ex]

Phys. Rev. D 81, 032002 (2010)

352. "First Measurement of the b-jet Cross Section in Events with a W Boson in $\mathbf{p}$ anti-p Collisions at $\mathrm{s}^{* *}(1 / 2)=1.96-\mathrm{TeV}$ "

T. Aaltonen et al. [CDF Collaboration].

arXiv:0909.1505 [hep-ex]

Phys. Rev. Lett. 104, 131801 (2010)

353. "A Search for the Higgs Boson Produced in Association with $\mathrm{Z}-i$ l+ l- Using the Matrix Element Method at CDF II"

T. Aaltonen et al. [CDF Collaboration].

arXiv:0908.3534 [hep-ex]

Phys. Rev. D 80, 071101 (2009)

354. "The CMS barrel calorimeter response to particle beams from 2-GeV/c to 350$\mathrm{GeV} / \mathrm{c} "$

S. Abdullin et al. [USCMS and ECAL/HCAL Collaborations].

Eur. Phys. J. C 60, 359 (2009), [Erratum-ibid. C 61, 353 (2009)].

355. "Search for charged Higgs bosons in decays of top quarks in $p$ anti-p collisions at $\mathrm{s}^{* *}(1 / 2)=1.96 \mathrm{TeV}^{\prime \prime}$

T. Aaltonen et al. [CDF Collaboration].

arXiv:0907.1269 [hep-ex]

Phys. Rev. Lett. 103, 101803 (2009)

356. "A Search for the Associated Production of the Standard-Model Higgs Boson in the All-Hadronic Channel"

T. Aaltonen et al. [CDF Collaboration].

arXiv:0907.0810 [hep-ex]

Phys. Rev. Lett. 103, 221801 (2009)

357. "Search for a Higgs Boson produced in association a W Boson in p anti-p Collisions at $\mathrm{s}^{* *}(1 / 2)=1.96-\mathrm{TeV} "$

T. Aaltonen et al. [CDF Collaboration].

arXiv:0906.5613 [hep-ex]

Phys. Rev. Lett. 103, 101802 (2009)

358. "Measurement of the Top Quark Mass Using the Invariant Mass of Lepton Pairs in Soft Muon b-tagged Events"

T. Aaltonen et al. [CDF Collaboration].

arXiv:0906.5371 [hep-ex]

Phys. Rev. D 80, 051104 (2009)

359. "Precision Measurement of the X(3872) Mass in J/psi pi+ pi- Decays"

T. Aaltonen et al. [CDF Collaboration].

arXiv:0906.5218 [hep-ex]

Phys. Rev. Lett. 103, 152001 (2009) 
360. "Search for Higgs bosons predicted in two-Higgs-doublet models via decays to tau lepton pairs in $1.96-\mathrm{TeV}$ p anti-p collisions"

T. Aaltonen et al. [CDF Collaboration].

arXiv:0906.1014 [hep-ex]

Phys. Rev. Lett. 103, 201801 (2009)

361. "Searching the Inclusive Lepton + Photon + Missing $\mathbf{E}(\mathbf{T})+$ b-quark Signature for Radiative Top Quark Decay and Non-Standard-Model Processes"

T. Aaltonen et al. [CDF Collaboration].

arXiv:0906.0518 [hep-ex]

Phys. Rev. D 80, 011102 (2009)

362. "First Observation of Vector Boson Pairs in a Hadronic Final State at the Tevatron Collider"

T. Aaltonen et al. [CDF Collaboration].

arXiv:0905.4714 [hep-ex]

Phys. Rev. Lett. 103, 091803 (2009)

363. "Search for Standard Model Higgs Boson Production in Association with a W Boson using a Neural Network Discriminant at CDF"

T. Aaltonen et al. [CDF Collaboration].

arXiv:0905.3155 [hep-ex]

Phys. Rev. D 80, 012002 (2009)

364. "Observation of the Omega(b)- Baryon and Measurement of the Properties of the $\mathrm{Xi}(\mathrm{b})$ - and Omega(b)- Baryons"

T. Aaltonen et al. [CDF Collaboration].

arXiv:0905.3123 [hep-ex]

Phys. Rev. D 80, 072003 (2009)

365. "Production of psi(2S) Mesons in p anti-p Collisions at 1.96-TeV"

T. Aaltonen et al. [CDF Collaboration].

arXiv:0905.1982 [hep-ex]

Phys. Rev. D 80, 031103 (2009)

366. "Search for a Fermiophobic Higgs Boson Decaying into Diphotons in p anti-p Collisions at $\mathrm{s}^{* *}(1 / 2)=1.96-\mathrm{TeV} "$

T. Aaltonen et al. [CDF Collaboration].

arXiv:0905.0413 [hep-ex]

Phys. Rev. Lett. 103, 061803 (2009)

367. "Search for the Neutral Current Top Quark Decay t - i Zc Using Ratio of ZBoson + 4 Jets to W-Boson + 4 Jets Production"

T. Aaltonen et al. [CDF Collaboration].

arXiv:0905.0277 [hep-ex]

Phys. Rev. D 80, 052001 (2009) 
368. "Search for Anomalous Production of Events with a Photon, Jet, b-quark Jet, and Missing Transverse Energy"

T. Aaltonen et al. [CDF Collaboration].

arXiv:0905.0231 [hep-ex]

Phys. Rev. D 80, 052003 (2009)

369. "Measurement of Particle Production and Inclusive Differential Cross Sections in $p$ anti-p Collisions at $\mathrm{s}^{* *}(1 / 2)=1.96-\mathrm{TeV} "$

T. Aaltonen et al. [CDF Collaboration].

arXiv:0904.1098 [hep-ex]

Phys. Rev. D 79, 112005 (2009), [Erratum-ibid. D 82, 119903 (2010)]

370. "A Measurement of the $t$ anti-t Cross Section in $p$ anti-p Collisions at $s^{* *}(1 / 2)$ = 1.96-TeV using Dilepton Events with a Lepton plus Track Selection"

T. Aaltonen et al. [CDF Collaboration].

arXiv:0903.5263 [hep-ex]

Phys. Rev. D 79, 112007 (2009)

371. "First Measurement of the $t$ anti-t Differential Cross Section d sigma/dM(t antit) in $\mathrm{p}$ anti-p Collisions at $\mathrm{s}^{* *}(1 / 2)=1.96-\mathrm{TeV}^{\prime \prime}$

T. Aaltonen et al. [CDF Collaboration].

arXiv:0903.2850 [hep-ex]

Phys. Rev. Lett. 102, 222003 (2009)

372. "Search for Gluino-Mediated Sbottom Production in p anti-p Collisions at s**(1/2)

$=1.96-\mathrm{TeV}^{\prime \prime}$

T. Aaltonen et al. [CDF Collaboration].

arXiv:0903.2618 [hep-ex]

Phys. Rev. Lett. 102, 221801 (2009)

373. "Measurement of the b-Hadron Production Cross Section Using Decays to MUd0 X Final States in p anti-p Collisions at $s^{* *}(1 / 2)=1.96-\mathrm{TeV}^{\prime}$

T. Aaltonen et al. [CDF Collaboration].

arXiv:0903.2403 [hep-ex]

Phys. Rev. D 79, 092003 (2009)

374. "Evidence for a Narrow Near-Threshold Structure in the $J / \psi \phi$ Mass Spectrum in $B^{+} \rightarrow J / \psi \phi K^{+}$Decays"

T. Aaltonen et al. [CDF Collaboration].

arXiv:0903.2229 [hep-ex]

Phys. Rev. Lett. 102, 242002 (2009)

375. "Search for narrow resonances lighter than Upsilon mesons"

T. Aaltonen et al. [CDF Collaboration].

arXiv:0903.2060 [hep-ex]

Eur. Phys. J. C 62, 319 (2009) 
376. "First Observation of Electroweak Single Top Quark Production"

T. Aaltonen et al. [CDF Collaboration].

arXiv:0903.0885 [hep-ex]

Phys. Rev. Lett. 103, 092002 (2009)

377. "Search for WW and WZ production in lepton plus jets final state at CDF" T. Aaltonen et al. [CDF Collaboration].

arXiv:0903.0814 [hep-ex]

Phys. Rev. D 79, 112011 (2009)

378. "Search for the Production of Narrow t anti-b Resonances in $1.9 \mathrm{fb}-1$ of $p$ anti-p Collisions at $\mathrm{s}^{* *}(1 / 2)=1.96-\mathrm{TeV} "$

T. Aaltonen et al. [CDF Collaboration].

arXiv:0902.3276 [hep-ex]

Phys. Rev. Lett. 103, 041801 (2009)

379. "Search for exclusive $Z$ boson production and observation of high mass $p \bar{p} \rightarrow$ $\gamma \gamma \rightarrow p+\ell \ell+\bar{p}$ events in $p \bar{p}$ collisions at $\sqrt{s}=1.96 \mathbf{T e V}^{\prime \prime}$

T. Aaltonen et al. [CDF Collaboration].

arXiv:0902.2816 [hep-ex]

Phys. Rev. Lett. 102, 222002 (2009)

380. "Observation of exclusive charmonium production and gamma gamma $-\mathbf{i} \mathbf{m u}+\mathbf{m u}-$ in $\mathrm{p}$ anti-p collisions at $\mathrm{s}^{* *}(1 / 2)=1.96-\mathrm{TeV}^{\prime}$

T. Aaltonen et al. [CDF Collaboration].

arXiv:0902.1271 [hep-ex]

Phys. Rev. Lett. 102, 242001 (2009)

381. "Search for Long-Lived Massive Charged Particles in 1.96 TeV $\bar{p} p$ Collisions"

T. Aaltonen et al. [CDF Collaboration].

arXiv:0902.1266 [hep-ex]

Phys. Rev. Lett. 103, 021802 (2009)

382. "Measurement of the $t \bar{t}$ Production Cross Section in $\mathbf{2} \mathrm{fb}^{-1}$ of $p \bar{p}$ Collisions at $\sqrt{s}=1.96$ TeV Using Lepton Plus Jets Events with Soft Muon b-Tagging"

T. Aaltonen et al. [CDF Collaboration].

arXiv:0901.4142 [hep-ex]

Phys. Rev. D 79, 052007 (2009)

383. "Search for the Decays B0(s) - $\mathbf{i}$ e+ mu- and B0(s) - i e+ e- in CDF Run II" T. Aaltonen et al. [CDF Collaboration].

arXiv:0901.3803 [hep-ex]

Phys. Rev. Lett. 102, 201801 (2009)

384. "Measurement of the top quark mass at CDF using the 'neutrino $\phi$ weighting' template method on a lepton plus isolated track sample"

T. Aaltonen et al. [CDF Collaboration]. 
arXiv:0901.3773 [hep-ex]

Phys. Rev. D 79, 072005 (2009)

385. "Direct Measurement of the $W$ Production Charge Asymmetry in $p \bar{p}$ Collisions at $\sqrt{s}=1.96 \mathbf{T e V}^{\prime}$

T. Aaltonen et al. [CDF Collaboration].

arXiv:0901.2169 [hep-ex]

Phys. Rev. Lett. 102, 181801 (2009)

386. "Top Quark Mass Measurement in the Lepton plus Jets Channel Using a Modified Matrix Element Method"

T. Aaltonen et al. [CDF Collaboration].

arXiv:0812.4469 [hep-ex]

Phys. Rev. D 79, 072001 (2009)

387. "Measurement of Cross Sections for $b$ Jet Production in Events with a $Z$ Boson in $p^{-}$anti-p Collisions at $\sqrt{s}=1.96-\mathbf{T e V}^{\prime}$

T. Aaltonen et al. [CDF Collaboration].

arXiv:0812.4458 [hep-ex]

Phys. Rev. D 79, 052008 (2009)

388. "Observation of New Charmless Decays of Bottom Hadrons"

T. Aaltonen et al. [CDF Collaboration].

arXiv:0812.4271 [hep-ex]

Phys. Rev. Lett. 103, 031801 (2009)

389. "Search for new particles decaying into dijets in proton-antiproton collisions at $\mathrm{s}^{* *}(1 / 2)=1.96-\mathrm{TeV} "$

T. Aaltonen et al. [CDF Collaboration].

arXiv:0812.4036 [hep-ex]

Phys. Rev. D 79, 112002 (2009)

390. "Search for top-quark production via flavor-changing neutral currents in $W+1$ jet events at CDF"

T. Aaltonen et al. [CDF Collaboration].

arXiv:0812.3400 [hep-ex]

Phys. Rev. Lett. 102, 151801 (2009)

391. "Measurement of the $k_{T}$ Distribution of Particles in Jets Produced in $p \bar{p}$ Collisions at $\sqrt{s}=1.96-\mathrm{TeV} "$

T. Aaltonen et al. [CDF Collaboration].

arXiv:0811.2820 [hep-ex]

Phys. Rev. Lett. 102, 232002 (2009)

392. "Inclusive Search for Squark and Gluino Production in $p \bar{p}$ Collisions at $\sqrt{s}=$ 1.96-TeV"

T. Aaltonen et al. [CDF Collaboration]. 
arXiv:0811.2512 [hep-ex]

Phys. Rev. Lett. 102, 121801 (2009)

393. "Design, performance, and calibration of the CMS Hadron-outer calorimeter" S. Abdullin et al. [CMS HCAL Collaboration].

Eur. Phys. J. C 57, 653 (2008).

394. "Top Quark Mass Measurement in the $t \bar{t}$ All Hadronic Channel using a Matrix Element Technique in $p \bar{p}$ Collisions at $\sqrt{s}=1.96-\mathrm{TeV} "$

T. Aaltonen et al. [CDF Collaboration].

arXiv:0811.1062 [hep-ex]

Phys. Rev. D 79, 072010 (2009)

395. "Measurement of W-Boson Helicity Fractions in Top-Quark Decays Using cos theta*"

T. Aaltonen et al. [CDF Collaboration].

arXiv:0811.0344 [hep-ex]

Phys. Lett. B 674, 160 (2009)

396. "A Search for high-mass resonances decaying to dimuons at CDF"

T. Aaltonen et al. [CDF Collaboration].

arXiv:0811.0053 [hep-ex]

Phys. Rev. Lett. 102, 091805 (2009)

397. "Search for new physics in the $\mathbf{m u} \mathbf{m u}+\mathbf{e} / \mathbf{m u}+\mathbf{E}(\mathbf{T})$ channel with a low-p(T) lepton threshold at the Collider Detector at Fermilab"

T. Aaltonen et al. [CDF Collaboration].

arXiv:0810.3522 [hep-ex]

Phys. Rev. D 79, 052004 (2009)

398. "First Measurement of the Ratio of Branching Fractions $B\left(\Lambda_{b}^{0} \rightarrow \Lambda_{c}^{+} \mu^{-} \bar{\nu}_{\mu} / B\left(\operatorname{Lambda} a_{b}^{0} \rightarrow\right.\right.$ $\left.\Lambda_{c}^{+} \pi^{-}\right) "$

T. Aaltonen et al. [CDF Collaboration].

arXiv:0810.3213 [hep-ex]

Phys. Rev. D 79, 032001 (2009)

399. "Search for High-Mass $e^{+}$e- Resonances in $p \bar{p}$ Collisions at $\sqrt{s}=1.96-\mathrm{TeV}$ "

T. Aaltonen et al. [CDF Collaboration].

arXiv:0810.2059 [hep-ex]

Phys. Rev. Lett. 102, 031801 (2009)

400. "Measurement of Resonance Parameters of Orbitally Excited Narrow $B^{0}$ Mesons" T. Aaltonen et al. [CDF Collaboration].

arXiv:0809.5007 [hep-ex]

Phys. Rev. Lett. 102, 102003 (2009)

401. "Search for Maximal Flavor Violating Scalars in Same-Charge Lepton Pairs in $p \bar{p}$ Collisions at $\sqrt{s}=1.96-\mathrm{TeV}^{\prime}$ 
T. Aaltonen et al. [CDF Collaboration].

arXiv:0809.4903 [hep-ex]

Phys. Rev. Lett. 102, 041801 (2009)

402. "First simultaneous measurement of the top quark mass in the lepton + jets and dilepton channels at CDF"

T. Aaltonen et al. [CDF Collaboration].

arXiv:0809.4808 [hep-ex]

Phys. Rev. D 79, 092005 (2009)

403. "Search for a Higgs Boson Decaying to Two $W$ Bosons at CDF"

T. Aaltonen et al. [CDF Collaboration].

arXiv:0809.3930 [hep-ex]

Phys. Rev. Lett. 102, 021802 (2009)

404. "Global Search for New Physics with 2.0 $\mathrm{fb}^{* *}(-1)$ at CDF"

T. Aaltonen et al. [CDF Collaboration].

arXiv:0809.3781 [hep-ex]

Phys. Rev. D 79, 011101 (2009)

405. "The CMS experiment at the CERN LHC"

S. Chatrchyan et al. [CMS Collaboration].

JINST 3, S08004 (2008).

406. "Measurement of the Single Top Quark Production Cross Section at CDF"

T. Aaltonen et al. [CDF Collaboration].

arXiv:0809.2581 [hep-ex]

Phys. Rev. Lett. 101, 252001 (2008)

407. "First observation of $\bar{B}_{s}^{0} \rightarrow D_{s}^{ \pm} K^{\mp}$ and measurement of the ratio of branching fractions $\mathbf{B}\left(\bar{B}_{s}^{0} \rightarrow D_{s}^{ \pm} K^{\mp} / \mathbf{B}\left(\bar{B}_{s}^{0} \rightarrow D_{s}^{+} \pi^{-}\right)\right.$"

T. Aaltonen et al. [CDF Collaboration].

arXiv:0809.0080 [hep-ex]

Phys. Rev. Lett. 103, 191802 (2009)

408. "Search for Supersymmetry in $p \bar{p}$ Collisions at $\sqrt{s}=1.96-\mathrm{TeV}$ Using the Trilepton Signature of Chargino-Neutralino Production"

T. Aaltonen et al. [CDF Collaboration].

arXiv:0808.2446 [hep-ex]

Phys. Rev. Lett. 101, 251801 (2008)

409. "First Direct Bound on the Total Width of the Top Quark in $p \bar{p}$ Collisions at $\sqrt{s}=1.96-\mathrm{TeV}^{\prime \prime}$

T. Aaltonen et al. [CDF Collaboration].

arXiv:0808.2167 [hep-ex]

Phys. Rev. Lett. 102, 042001 (2009) 
410. "Search for Doubly Charged Higgs Bosons with Lepton-Flavor-Violating Decays involving Tau Leptons"

T. Aaltonen et al. [CDF Collaboration].

arXiv:0808.2161 [hep-ex]

Phys. Rev. Lett. 101, 121801 (2008)

411. "Measurement of the top quark mass with dilepton events selected using neuroevolution at CDF"

T. Aaltonen et al. [CDF Collaboration].

arXiv:0807.4652 [hep-ex]

Phys. Rev. Lett. 102, 152001 (2009)

412. "Search for the Higgs boson produced with $Z \rightarrow \ell^{+} \ell^{-}$in $p \bar{p}$ collisions at $\sqrt{s}=\mathbf{1 . 9 6}$ TeV"

T. Aaltonen et al. [CDF Collaboration].

arXiv:0807.4493 [hep-ex]

Phys. Rev. Lett. 101, 251803 (2008)

413. "Measurement of the fraction of $t \bar{t}$ production via gluon-gluon fusion in $p \bar{p}$ collisions at $\sqrt{s}=1.96-\mathrm{TeV}^{\prime}$

T. Aaltonen et al. [CDF Collaboration].

arXiv:0807.4262 [hep-ex]

Phys. Rev. D 79, 031101 (2009)

414. "Search for large extra dimensions in final states containing one photon or jet and large missing transverse energy produced in $p \bar{p}$ collisions at $\sqrt{s}=1.96-\mathrm{TeV}$ " T. Aaltonen et al. [CDF Collaboration].

arXiv:0807.3132 [hep-ex]

Phys. Rev. Lett. 101, 181602 (2008)

415. "Measurement of the Inclusive Jet Cross Section at the Fermilab Tevatron $\mathbf{p}$ anti-p Collider Using a Cone-Based Jet Algorithm"

T. Aaltonen et al. [CDF Collaboration].

arXiv:0807.2204 [hep-ex]

Phys. Rev. D 78, 052006 (2008), [Erratum-ibid. D 79, 119902 (2009)]

416. "Design, performance, and calibration of CMS hadron-barrel calorimeter wedges" S. Abdullin et al. [CMS HCAL Collaboration].

Eur. Phys. J. C 55, 159 (2008).

417. "Forward-Backward Asymmetry in Top Quark Production in $p \bar{p}$ Collisions at sqrts $=1.96 \mathbf{T e V}$ "

T. Aaltonen et al. [CDF Collaboration].

arXiv:0806.2472 [hep-ex]

Phys. Rev. Lett. 101, 202001 (2008)

418. "Measurement of $b$-jet Shapes in Inclusive Jet Production in $p \bar{p}$ Collisions at $\sqrt{s}$ $=1.96-\mathrm{TeV}^{\prime}$ 
T. Aaltonen et al. [CDF Collaboration].

arXiv:0806.1699 [hep-ex]

Phys. Rev. D 78, 072005 (2008)

419. "Design, performance, and calibration of CMS hadron endcap calorimeters"

G. Baiatian et al. [CMS HCAL Collaboration].

420. "Search for the Flavor Changing Neutral Current Decay $t \rightarrow Z q$ in $p \bar{p}$ Collisions at $\sqrt{s}=1.96 \mathbf{T e V}^{\prime}$

T. Aaltonen et al. [CDF Collaboration].

arXiv:0805.2109 [hep-ex]

Phys. Rev. Lett. 101, 192002 (2008)

421. "Search for the Rare Decays $B^{+} \rightarrow \mu^{+} \mu^{-} K^{+}, B^{0} \rightarrow \mu^{+} \mu^{-} K^{* 0}(892)$, and $B_{s}^{0} \rightarrow \mu^{+} \mu^{-} \phi$ at CDF"

T. Aaltonen et al. [CDF Collaboration].

arXiv:0804.3908 [hep-ex]

Phys. Rev. D 79, 011104 (2009)

422. "Search for Heavy, Long-Lived Neutralinos that Decay to Photons at CDF II Using Photon Timing"

T. Aaltonen et al. [CDF Collaboration].

arXiv:0804.1043 [hep-ex]

Phys. Rev. D 78, 032015 (2008)

423. "Search for Hadronic Decays of $W$ and $Z$ Bosons in Photon Events in $p \bar{p}$ Collisions at $\sqrt{s}=1.96-\mathrm{GeV}^{\prime \prime}$

T. Aaltonen et al. [CDF Collaboration].

arXiv:0803.4264 [hep-ex]

Phys. Rev. D 80, 052011 (2009)

424. "Search for Standard Model Higgs Boson Production in Association with a $W$ Boson at CDF"

T. Aaltonen et al. [CDF Collaboration].

arXiv:0803.3493 [hep-ex]

Phys. Rev. D 78, 032008 (2008)

425. "Search for Pair Production of Scalar Top Quarks Decaying to a $\tau$ Lepton and a $b$ Quark in $p \bar{p}$ Collisions at sqrts $=1.96$ TeV"

T. Aaltonen et al. [CDF Collaboration].

arXiv:0802.3887 [hep-ex]

Phys. Rev. Lett. 101, 071802 (2008)

426. "Two-Particle Momentum Correlations in Jets Produced in $p \bar{p}$ Collisions at $\sqrt{s}$ $=1.96-\mathrm{TeV} "$

T. Aaltonen et al. [CDF Collaboration].

arXiv:0802.3182 [hep-ex]

Phys. Rev. D 77, 092001 (2008) 
427. "Search for the Higgs boson in events with missing transverse energy and $b$ quark jets produced in proton-antiproton collisions at $\sqrt{s}=1.96 \mathrm{TeV}$ "

T. Aaltonen et al. [CDF Collaboration].

arXiv:0802.0432 [hep-ex]

Phys. Rev. Lett. 100, 211801 (2008)

428. "First Measurement of $Z Z$ Production in panti-p Collisions at $\sqrt{s}=1.96-\mathrm{TeV}^{\prime}$ T. Aaltonen et al. [CDF Collaboration].

arXiv:0801.4806 [hep-ex]

Phys. Rev. Lett. 100, 201801 (2008)

429. "Measurement of Ratios of Fragmentation Fractions for Bottom Hadrons in $p \bar{p}$ Collisions at $\sqrt{s}=1.96-\mathrm{TeV}^{\prime \prime}$

T. Aaltonen et al. [CDF Collaboration].

arXiv:0801.4375 [hep-ex]

Phys. Rev. D 77, 072003 (2008)

430. "Search for Heavy Top-like Quarks Using Lepton Plus Jets Events in 1.96-TeV $p \bar{p}$ Collisions"

T. Aaltonen et al. [CDF Collaboration].

arXiv:0801.3877 [hep-ex]

Phys. Rev. Lett. 100, 161803 (2008)

431. "Search for New Heavy Particles Decaying to $Z^{0} Z^{0} \rightarrow$ eeee in $p-\bar{p}$ Collisions at $\sqrt{s}=1.96-\mathrm{TeV}^{\prime \prime}$

T. Aaltonen et al. [CDF Collaboration].

arXiv:0801.1129 [hep-ex]

Phys. Rev. D 78, 012008 (2008)

432. "Measurement of the Nucleon Strange-Antistrange Asymmetry at Next-to-Leading Order in QCD from NuTeV Dimuon Data"

D. Mason et al. [NuTeV Collaboration].

Phys. Rev. Lett. 99, 192001 (2007).

433. "First measurement of the fraction of top quark pair production through gluongluon fusion"

T. Aaltonen et al. [CDF Collaboration].

arXiv:0712.3273 [hep-ex]

Phys. Rev. D 78, 111101 (2008)

434. "Model-Independent Global Search for New High-p(T) Physics at CDF"

T. Aaltonen et al. [CDF Collaboration].

arXiv:0712.2534 [hep-ex]

435. "First Flavor-Tagged Determination of Bounds on Mixing-Induced CP Violation in $B_{s}^{0} \rightarrow J / \psi \phi$ Decays"

T. Aaltonen et al. [CDF Collaboration]. 
arXiv:0712.2397 [hep-ex]

Phys. Rev. Lett. 100, 161802 (2008)

436. "Measurement of lifetime and decay-width difference in $B_{s}^{0} \rightarrow J / \psi \phi$ decays"

T. Aaltonen et al. [CDF Collaboration].

arXiv:0712.2348 [hep-ex]

Phys. Rev. Lett. 100, 121803 (2008)

437. "d sigma/dy Distribution of Drell-Yan Dielectron Pairs"

J. Han, A. Bodek, W. Sakumoto and Y. Chung.

J. Phys. Conf. Ser. 110, 042009 (2008).

438. "Evidence for $D^{0}-\bar{D}^{0}$ mixing using the CDF II Detector"

T. Aaltonen et al. [CDF Collaboration].

arXiv:0712.1567 [hep-ex]

Phys. Rev. Lett. 100, 121802 (2008)

439. "Search for $B_{s}^{0} \rightarrow \mu^{+} \mu^{-}$and $B_{d}^{0} \rightarrow \mu^{+} \mu^{-}$decays with $2 f b^{-1}$ of $p \bar{p}$ collisions"

T. Aaltonen et al. [CDF Collaboration].

arXiv:0712.1708 [hep-ex]

Phys. Rev. Lett. 100, 101802 (2008)

440. "Observation of the Decay $B^{+}-(c) \rightarrow J / \psi \pi^{ \pm}$and Measurement of the $B^{+}-(c)$ Mass"

T. Aaltonen et al. [CDF Collaboration].

arXiv:0712.1506 [hep-ex]

Phys. Rev. Lett. 100, 182002 (2008)

441. "Model-Independent and Quasi-Model-Independent Search for New Physics at CDF"

T. Aaltonen et al. [CDF Collaboration].

arXiv:0712.1311 [hep-ex]

Phys. Rev. D 78, 012002 (2008)

442. "Observation of Exclusive Dijet Production at the Fermilab Tevatron $p^{-} \bar{p}$ Collider"

T. Aaltonen et al. [CDF Collaboration].

arXiv:0712.0604 [hep-ex]

Phys. Rev. D 77, 052004 (2008)

443. "A unified approach to e / nu - $\mathbf{N}$ deep inelastic scattering cross sections at all $\mathrm{Q}^{* *} 2$ "

A. Bodek and U. -k. Yang.

444. "Measurement of the cross section for $W^{-}$boson production in association with jets in $p \bar{p}$ collisions at $\sqrt{s}=1.96-\mathrm{TeV}^{\prime \prime}$

T. Aaltonen et al. [CDF Collaboration].

arXiv:0711.4044 [hep-ex]

Phys. Rev. D 77, 011108 (2008) 
445. "Measurement of inclusive jet cross-sections in $\mathbf{Z} /$ gamma* $^{*}\left(-i e^{+} e^{-)}+\right.$jets production in $p \bar{p}$ collisions at $\sqrt{s}=1.96-\mathrm{TeV}^{\prime \prime}$

T. Aaltonen et al. [CDF Collaboration].

arXiv:0711.3717 [hep-ex]

Phys. Rev. Lett. 100, 102001 (2008)

446. "Search for chargino-neutralino production in $p \bar{p}$ collisions at 1.96-TeV with high$\mathbf{p}(T)$ leptons"

T. Aaltonen et al. [CDF Collaboration].

arXiv:0711.3161 [hep-ex]

Phys. Rev. D 77, 052002 (2008)

447. "First measurement of the production of a $W$ boson in association with a single charm quark in $p \bar{p}$ collisions at $\sqrt{s}=1.96-\mathrm{TeV}^{\prime \prime}$

T. Aaltonen et al. [CDF Collaboration].

arXiv:0711.2901 [hep-ex]

Phys. Rev. Lett. 100, 091803 (2008)

448. "New analysis technique to measure the $W$ production charge asymmetry at the Fermilab Tevatron"

A. Bodek, Y. Chung, B. -Y. Han, K. S. McFarland and E. Halkiadakis. arXiv:0711.2859 [hep-ph]

Phys. Rev. D 77, 111301 (2008)

449. "Limits on the production of narrow $t \bar{t}$ resonances in $p \bar{p}$ collisions at $\sqrt{s}=1.96$ TeV"

T. Aaltonen et al. [CDF Collaboration].

arXiv:0710.5335 [hep-ex]

Phys. Rev. D 77, 051102 (2008)

450. "Search for Standard Model Higgs Bosons Produced in Association with $W$ Bosons"

T. Aaltonen et al. [CDF Collaboration].

arXiv:0710.4363 [hep-ex]

Phys. Rev. Lett. 100, 041801 (2008)

451. "Observation of orbitally excited $B_{s}$ mesons"

T. Aaltonen et al. [CDF Collaboration].

arXiv:0710.4199 [hep-ex]

Phys. Rev. Lett. 100, 082001 (2008)

452. "CMS technical design report, volume II: Physics performance"

G. L. Bayatian et al. [CMS Collaboration].

J. Phys. G G 34, 995 (2007).

453. "A Direct measurement of the $W$ boson width in $p \bar{p}$ collisions at $\sqrt{s}=\mathbf{1 . 9 6}-\mathbf{T e V}$ " T. Aaltonen et al. [CDF Collaboration]. 
arXiv:0710.4112 [hep-ex]

Phys. Rev. Lett. 100, 071801 (2008)

454. "Cross-section constrained top quark mass measurement from dilepton events at the Tevatron"

T. Aaltonen et al. [CDF Collaboration].

arXiv:0710.4037 [hep-ex]

Phys. Rev. Lett. 100, 062005 (2008)

455. "First Observation of the Decay $B_{s}^{0} \rightarrow D_{s}^{-} D_{s}^{+}$and Measurement of Its Branching Ratio"

T. Aaltonen et al. [CDF Collaboration].

Phys. Rev. Lett. 100, 021803 (2008).

456. "Measurement of correlated $b^{-} \bar{b}$ production in $p^{-} \bar{p}$ collisions at $\sqrt{s}=1960 \mathrm{GeV}^{\text {" }}$ T. Aaltonen et al. [CDF Collaboration].

arXiv:0710.1895 [hep-ex]

Phys. Rev. D 77, 072004 (2008)

457. "Muon internal bremsstrahlung: A Conventional explanation for the excess nu(e) events in MiniBoone"

A. Bodek.

arXiv:0709.4004 [hep-ex]

458. "Extraction of the axial nucleon form-factor from neutrino experiments on deuterium"

A. Bodek, S. Avvakumov, R. Bradford and H. S. Budd.

arXiv:0709.3538 [hep-ex]

J. Phys. Conf. Ser. 110, 082004 (2008)

459. "Synchronization and timing in CMS HCAL"

G. Bayatian et al. [CMS Collaboration].

460. "Search for resonant $t \bar{t}$ production in $p \bar{p}$ collisions at $\sqrt{s}=1.96-\mathbf{T e V}$ "

T. Aaltonen et al. [CDF Collaboration].

arXiv:0709.0705 [hep-ex]

Phys. Rev. Lett. 100, 231801 (2008)

461. "First Run II Measurement of the $W$ Boson Mass"

T. Aaltonen et al. [CDF Collaboration].

arXiv:0708.3642 [hep-ex]

Phys. Rev. D 77, 112001 (2008)

462. "Vector and Axial Nucleon Form Factors:A Duality Constrained Parameterization"

A. Bodek, S. Avvakumov, R. Bradford and H. S. Budd.

arXiv:0708.1946 [hep-ex]

Eur. Phys. J. C 53, 349 (2008) 
463. "Modeling Atmospheric Neutrino Interactions: Duality Constrained Parameterization of Vector and Axial Nucleon Form Factors"

A. Bodek, S. Avvakumov, R. Bradford and H. S. Budd.

arXiv:0708.1827 [hep-ex]

464. "Search for Direct Pair Production of Supersymmetric Top and Supersymmetric Bottom Quarks in $p \bar{p}$ Collisions at $\sqrt{s}=1.96-\mathrm{TeV} "$

T. Aaltonen et al. [CDF Collaboration].

arXiv:0707.2567 [hep-ex]

Phys. Rev. D 76, 072010 (2007)

465. "Search for new physics in high mass electron-positron events in $p \bar{p}$ collisions at $\sqrt{s}=1.96-\mathrm{TeV}^{\prime}$

T. Aaltonen et al. [CDF Collaboration].

arXiv:0707.2524 [hep-ex]

Phys. Rev. Lett. 99, 171802 (2007)

466. "Search for exclusive $\gamma \gamma$ production in hadron-hadron collisions"

T. Aaltonen et al. [CDF Collaboration].

arXiv:0707.2374 [hep-ex]

Phys. Rev. Lett. 99, 242002 (2007)

467. "Search for chargino-neutralino production in $p \bar{p}$ collisions at $\sqrt{s}=1.96-\mathrm{TeV}$ "

T. Aaltonen et al. [CDF Collaboration].

arXiv:0707.2362 [hep-ex]

Phys. Rev. Lett. 99, 191806 (2007)

468. "Search for a high-mass diphoton state and limits on Randall-Sundrum gravitons at CDF"

T. Aaltonen et al. [CDF Collaboration].

arXiv:0707.2294 [hep-ex]

Phys. Rev. Lett. 99, 171801 (2007)

469. "Observation and mass measurement of the baryon $X i_{b}^{-}$"

T. Aaltonen et al. [CDF Collaboration].

arXiv:0707.0589 [hep-ex]

Phys. Rev. Lett. 99, 052002 (2007)

470. "First measurement of the $W$ boson mass in run II of the Tevatron"

T. Aaltonen et al. [CDF Collaboration].

arXiv:0707.0085 [hep-ex]

Phys. Rev. Lett. 99, 151801 (2007)

471. "CMS expression of interest in the SLHC"

J. Nash, (Ed.) et al. [CMS Collaboration].

472. "CMS physics technical design report: Addendum on high density QCD with heavy ions" 
D. G. d'Enterria, (Ed.) et al. [CMS Collaboration].

J. Phys. G G 34, 2307 (2007).

473. "First observation of heavy baryons $\Sigma_{b}$ and $\Sigma_{b}^{*} "$

T. Aaltonen et al. [CDF Collaboration].

arXiv:0706.3868 [hep-ex]

Phys. Rev. Lett. 99, 202001 (2007)

474. "Measurement of the $p \bar{p} \rightarrow t \bar{t}$ production cross- section and the top quark mass at $\sqrt{s}=1.96-\mathrm{TeV}$ in the all-hadronic decay mode"

T. Aaltonen et al. [CDF Collaboration].

arXiv:0706.3790 [hep-ex]

Phys. Rev. D 76, 072009 (2007)

475. "Search for New Particles Leading to $Z+$ jets Final States in $p \bar{p}$ Collisions at $\sqrt{s}$ $=1.96-\mathrm{TeV} "$

T. Aaltonen et al. [CDF Collaboration].

arXiv:0706.3264 [hep-ex]

Phys. Rev. D 76, 072006 (2007)

476. "Search for Third Generation Vector Leptoquarks in $p \bar{p}$ Collisions at $\sqrt{s}=1.96$ TeV"

T. Aaltonen et al. [CDF Collaboration].

arXiv:0706.2832 [hep-ex]

Phys. Rev. D 77, 091105 (2008)

477. "Limits on Anomalous Triple Gauge Couplings in $p \bar{p}$ Collisions at $\sqrt{s}=1.96$ TeV"

T. Aaltonen et al. [CDF Collaboration].

arXiv:0705.2247 [hep-ex]

Phys. Rev. D 76, 111103 (2007)

478. "Measurement of the top-quark mass using missing $E_{T}+$ jets events with secondary vertex $b$-tagging at CDF II"

T. Aaltonen et al. [CDF Collaboration].

arXiv:0705.1594 [hep-ex]

Phys. Rev. D 75, 111103 (2007)

479. "Search for heavy, long-lived particles that decay to photons at CDF II"

A. Abulencia et al. [CDF Collaboration].

arXiv:0704.0760 [hep-ex]

Phys. Rev. Lett. 99, 121801 (2007)

480. "Polarization of $J / \psi$ and $\psi_{2 S}$ mesons produced in $p \bar{p}$ collisions at $\sqrt{s}=1.96-\mathbf{T e V}$ " A. Abulencia et al. [CDF Collaboration].

arXiv:0704.0638 [hep-ex]

Phys. Rev. Lett. 99, 132001 (2007) 
481. "Precise measurement of the top quark mass in the lepton+jets topology at CDF II"

A. Abulencia et al. [CDF Collaboration].

hep-ex/0703045

Phys. Rev. Lett. 99, 182002 (2007)

482. "Measurement of $\sigma_{\chi_{c 2}} \mathcal{B}\left(\chi_{c 2} \rightarrow J / \psi \gamma\right) / \sigma_{\chi_{c 1}} \mathcal{B}\left(\chi_{c 1} \rightarrow J / \psi \gamma\right)$ in $p \bar{p}$ collisions at $\sqrt{s}=$ 1.96-TeV"

A. Abulencia et al. [CDF Collaboration].

hep-ex/0703028 [HEP-EX]

Phys. Rev. Lett. 98, 232001 (2007)

483. "Inclusive search for new physics with like-sign dilepton events in $p \bar{p}$ collisions at $\sqrt{s}=1.96-\mathrm{TeV}^{\prime \prime}$

A. Abulencia et al. [CDF Collaboration].

hep-ex/0702051

Phys. Rev. Lett. 98, 221803 (2007)

484. "Measurement of sigma $\mathrm{p}$ anti-p $-i \mathrm{Z}$. Br $(\mathrm{Z}-i$ 2tau $)$ in $\mathrm{p}$ anti-p collisions at $\mathrm{s}^{* *}(1 / 2)=1.96 \mathrm{TeV} "$

A. Abulencia et al. [CDF Collaboration].

Phys. Rev. D 75, 092004 (2007).

485. "First Measurement of the Ratio of Central-Electron to Forward-Electron W Partial Cross Sections in $p$ anti-p Collisions at $\mathrm{s}^{* *}(1 / 2)=1.96 \mathrm{TeV}^{\prime \prime}$

A. Abulencia et al. [CDF Collaboration].

hep-ex/0702037

Phys. Rev. Lett. 98, 251801 (2007)

486. "Search for new physics in lepton + photon $+X$ events with $929 \mathbf{p b}^{(-1)}$ of $p \bar{p}$ collisions at $\sqrt{s}=1.96-\mathrm{TeV}$ "

A. Abulencia et al. [CDF Collaboration].

hep-ex/0702029

Phys. Rev. D 75, 112001 (2007)

487. "Observation of $W Z$ Production"

A. Abulencia et al. [CDF Collaboration].

hep-ex/0702027

Phys. Rev. Lett. 98, 161801 (2007)

488. "Measurement of the Inclusive Jet Cross Section using the $k_{\mathrm{T}}$ algorithmin $p \bar{p}$ Collisions at $\sqrt{s}=1.96 \mathrm{TeV}$ with the CDF II Detector"

A. Abulencia et al. [CDF Collaboration].

hep-ex/0701051

Phys. Rev. D 75, 092006 (2007), [Erratum-ibid. D 75, 119901 (2007)]

489. "Search for anomalous production of multi-lepton events in $p \bar{p}$ collisions at $\sqrt{s}=$ 1.96-TeV" 
AAbulencia et al. [CDF Collaboration].

arXiv:0706.4448 [hep-ex]

Phys. Rev. Lett. 98, 131804 (2007)

490. "CMS physics: Technical design report"

G. L. Bayatian et al. [CMS Collaboration].

491. "Search for Exotic $\mathbf{S}=\mathbf{- 2}$ Baryons in proton-antiproton Collisions at $\operatorname{sqrt}(s)=$ $1.96 \mathrm{TeV} "$

A. Abulencia et al. [CDF Collaboration].

hep-ex/0612066

Phys. Rev. D 75, 032003 (2007)

492. "Measurement of the Top Quark Mass in $p \bar{p}$ Collisions at $\sqrt{s}=1.96$ TeV using the Decay Length Technique"

A. Abulencia et al. [CDF Collaboration].

hep-ex/0612061

Phys. Rev. D 75, 071102 (2007)

493. "Precision measurement of the top quark mass from dilepton events at CDF II" A. Abulencia et al. [CDF Collaboration].

hep-ex/0612060

Phys. Rev. D 75, 031105 (2007)

494. "Cross Section Measurements of High-p(T) Dilepton Final-State Processes Using a Global Fitting Method"

A. Abulencia et al. [CDF Collaboration].

hep-ex/0612058

Phys. Rev. D 78, 012003 (2008)

495. "Analysis of the quantum numbers $\mathrm{J}^{* *} \mathrm{PC}$ of the $\mathrm{X}(\mathbf{3 8 7 2})$ "

A. Abulencia et al. [CDF Collaboration].

hep-ex/0612053

Phys. Rev. Lett. 98, 132002 (2007)

496. "A unified model for inelastic e-N and $\mathrm{v}-\mathrm{N}$ cross sections at all $\mathrm{Q} *{ }^{*}$ "

A. Bodek and U. Yang.

AIP Conf. Proc. 842, 360 (2006).

497. "Measurement of the top-quark mass in all-hadronic decays in $\mathbf{p}$ anti-p collisions at CDF II"

T. Aaltonen et al. [CDF Collaboration].

hep-ex/0612026

Phys. Rev. Lett. 98, 142001 (2007)

498. "Measurement of the $\mathbf{B}+$ production cross-section in $\mathbf{p}$ anti-p collisions at $\mathbf{s}^{* *}(\mathbf{1} / \mathbf{2})$ $=1960-\mathrm{GeV}^{\prime}$

A. Abulencia et al. [CDF Collaboration]. 
hep-ex/0612015

Phys. Rev. D 75, 012010 (2007)

499. "Measurement of the Helicity Fractions of W Bosons from Top Quark Decays using Fully Reconstructed $t \bar{t}$ Events with CDF II"

A. Abulencia et al. [CDF Collaboration].

hep-ex/0612011

Phys. Rev. D 75, 052001 (2007)

500. "Observation of Exclusive Electron-Positron Production in Hadron-Hadron Collisions"

A. Abulencia et al. [CDF Collaboration].

hep-ex/0611040

Phys. Rev. Lett. 98, 112001 (2007)

501. "Search for W-prime boson decaying to electron-neutrino pairs in $\mathbf{p}$ anti-p collisions at $\mathrm{s}^{* *}(1 / 2)=1.96-\mathrm{TeV} "$

A. Abulencia et al. [CDF Collaboration].

hep-ex/0611022

Phys. Rev. D 75, 091101 (2007)

502. "Measurement of the Ratios of Branching Fractions B(Bo(s) - $\mathbf{i}$ D-(s) pi+ pi+ pi- $) / \mathrm{B}\left(\mathrm{B0}-_{i} \mathrm{D}-\mathrm{pi}+\mathrm{pi}+\mathrm{pi}-\right)$ and B(B0(s) - i D-(s) pi+) / B(B0 -i D- pi+)" A. Abulencia et al. [CDF Collaboration].

hep-ex/0610045

Phys. Rev. Lett. 98, 061802 (2007)

503. "Observation of B0(s) - anti-B0(s) Oscillations"

A. Abulencia et al. [CDF Collaboration].

hep-ex/0609040

Phys. Rev. Lett. 97, 242003 (2006)

504. "Measurement of the $\Lambda_{b}^{0}$ Lifetime in $\Lambda_{b}^{0} \rightarrow J / \psi \Lambda^{0}$ in $p \bar{p}$ Collisions at $\sqrt{s}=1.96$ TeV"

A. Abulencia et al. [CDF Collaboration].

hep-ex/0609021

Phys. Rev. Lett. 98, 122001 (2007)

505. "Search for $\mathrm{V}+\mathrm{A}$ current in top quark decay in $\mathrm{p}$ anti-p collisions at $\mathrm{s}^{* *}(1 / 2)=$ 1.96-TeV"

A. Abulencia et al. [CDF Collaboration].

hep-ex/0608062

Phys. Rev. Lett. 98, 072001 (2007)

506. "Measurement of the $t \bar{t}$ Production Cross Section in $p \bar{p}$ collisions at $\sqrt{s}=1.96$ TeV in the All Hadronic Decay Mode"

A. Abulencia et al. [CDF Collaboration]. 
hep-ex/0607095

Phys. Rev. D 74, 072005 (2006)

507. "Measurement of the $t \bar{t}$ Production Cross Section in $p \bar{p}$ collisions at $\sqrt{s}=1.96$ TeV using Lepton + Jets Events with Jet Probability $b^{-}$tagging"

A. Abulencia et al. [CDF Collaboration].

hep-ex/0607035

Phys. Rev. D 74, 072006 (2006)

508. "Observation of $B^{0}(s) \rightarrow K^{+} K^{-}$and Measurements of Branching Fractions of Charmless Two-body Decays of $B^{0}$ and $B_{s}^{0}$ Mesons in $\bar{p} p$ Collisions at $\sqrt{s}=1.96$ TeV"

A. Abulencia et al. [CDF Collaboration].

hep-ex/0607021

Phys. Rev. Lett. 97, 211802 (2006)

509. "Search for excited and exotic muons in the $\mu \gamma$ decay channel in $p \bar{p}$ collisions at $\sqrt{s}=1.96-\mathrm{TeV} "$

A. Abulencia et al. [CDF Collaboration].

hep-ex/0606043

Phys. Rev. Lett. 97, 191802 (2006)

510. "Measurement of the $B_{s}^{0}-\bar{B}_{s}^{0}$ Oscillation Frequency"

A. Abulencia et al. [CDF Collaboration].

hep-ex/0606027

Phys. Rev. Lett. 97, 062003 (2006)

511. "Measurement of the tanti-t Production Cross Section in $p$ anti-ptnipbar Collisions at $\sqrt{s}=1.96-\mathrm{TeV} "$

A. Abulencia et al. [CDF Collaboration].

hep-ex/0606017

Phys. Rev. Lett. 97, 082004 (2006)

512. "Search for a neutral Higgs boson decaying to a $W$ boson pair in $p$ antip collisions at $\sqrt{s}=1.96-\mathrm{TeV}^{\prime \prime}$

A. Abulencia et al. [CDF Collaboration].

hep-ex/0605124

Phys. Rev. Lett. 97, 081802 (2006)

513. "Top quark mass measurement from dilepton events at CDF II with the matrixelement method"

A. Abulencia et al. [CDF Collaboration]. hep-ex/0605118

Phys. Rev. D 74, 032009 (2006)

514. "Measurement of the $b$ jet cross-section in events with a $Z$ boson in $p \bar{p}$ collisions at $\sqrt{s}=1.96-\mathrm{TeV}^{\prime \prime}$

A. Abulencia et al. [CDF Collaboration]. 
hep-ex/0605099

Phys. Rev. D 74, 032008 (2006)

515. "Search for new physics in lepton + photon $+X$ events with $305 p b^{-1}$ of $p \bar{p}$ collisions at $\sqrt{s}=1.96-\mathrm{TeV}^{\prime \prime}$

A. Abulencia et al. [CDF Collaboration].

hep-ex/0605097

Phys. Rev. Lett. 97, 031801 (2006)

516. "Measurement of the ratio of branching fractions $\mathbf{B}\left(\mathbf{D} 0 \rightarrow K^{+} \pi^{-}\right) / \mathbf{B}\left(\mathbf{D} 0 \rightarrow K^{-} \pi^{+}\right)$ using the CDF II Detector"

A. Abulencia et al. [CDF Collaboration].

hep-ex/0605027

Phys. Rev. D 74, 031109 (2006)

517. "Search for Large Extra Dimensions in the Production of Jets and Missing Transverse Energy in p anti-p Collisions at $\mathrm{s}^{* *}(1 / 2)=1.96 \mathrm{TeV}^{\prime \prime}$

A. Abulencia et al. [CDF Collaboration].

hep-ex/0605101

Phys. Rev. Lett. 97, 171802 (2006)

518. "Measurement of the $\mathrm{t}$ anti-t production cross section in $\mathbf{p}$ anti-p collisions at $\mathrm{s}^{* *}(1 / 2)=1.96-\mathrm{TeV}$ using missing $\mathrm{E}(\mathrm{T})+$ jets events with secondary vertex b-tagging"

A. Abulencia et al. [CDF Collaboration].

hep-ex/0603043

Phys. Rev. Lett. 96, 202002 (2006)

519. "Measurement of the $\mathbf{B}(\mathbf{c})+$ meson lifetime using $\mathbf{B}(\mathbf{c})+-_{\mathbf{i}} \mathbf{J} / \mathbf{p s i}$ e+ nu(e)"

A. Abulencia et al. [CDF Collaboration].

hep-ex/0603027

Phys. Rev. Lett. 97, 012002 (2006)

520. "Search for high-mass resonances decaying to e mu in $p \bar{p}$ collisions at $\sqrt{s}=1.96$ TeV."

A. Abulencia et al. [CDF Collaboration].

hep-ex/0603006

Phys. Rev. Lett. 96, 211802 (2006)

521. "Search for $Z^{\prime} \rightarrow e^{+} e^{-}$using dielectron mass and angular distribution."

A. Abulencia et al. [CDF Collaboration].

hep-ex/0602045

Phys. Rev. Lett. 96, 211801 (2006)

522. "A New parameterization of the nucleon elastic form-factors"

R. Bradford, A. Bodek, H. S. Budd and J. Arrington.

hep-ex/0602017

Nucl. Phys. Proc. Suppl. 159, 127 (2006) 
523. "Measurement of the top quark mass using template methods on dilepton events in proton antiproton collisions at $\sqrt{s}=1.96-\mathrm{TeV} . "$

A. Abulencia et al. [CDF Collaboration].

hep-ex/0602008

Phys. Rev. D 73, 112006 (2006)

524. "Observation of $B_{s}^{0} \rightarrow \psi(2 S) \phi$ and measurement of ratio of branching fractions $B\left(B_{s}^{0} \rightarrow \psi(2 S) \phi\right) / B\left(B_{s}^{0} \rightarrow J / \psi \phi\right) "$

A. Abulencia et al. [CDF Collaboration].

hep-ex/0602005

Phys. Rev. Lett. 96, 231801 (2006)

525. "Measurement of $\sigma\left(\Lambda_{b}^{0}\right) / \sigma\left(\bar{B}^{0}\right) \times \mathrm{BR}\left(\Lambda_{b}^{0} \rightarrow \Lambda_{c}^{+} \pi^{-}\right) / \mathrm{BR}\left(\bar{B}^{0} \rightarrow D^{+} \pi^{-}\right)$in $p \bar{p}$ collisions at $\sqrt{s}=1.96-\mathrm{TeV}$."

A. Abulencia et al. [CDF Collaboration].

hep-ex/0601003

Phys. Rev. Lett. 98, 122002 (2007)

526. "Measurement of the dipion mass spectrum in $X(3872) \rightarrow J / \psi \pi^{+} \pi^{-}$decays."

A. Abulencia et al. [CDF Collaboration].

hep-ex/0512074

Phys. Rev. Lett. 96, 102002 (2006)

527. "A search for scalar bottom quarks from gluino decays in $\bar{p} p$ collisions at $\sqrt{s}=$ 1.96-TeV."

A. Abulencia et al. [CDF Collaboration].

hep-ex/0512072

Phys. Rev. Lett. 96, 171802 (2006)

528. "Top quark mass measurement from dilepton events at CDF II"

A. Abulencia et al. [CDF Collaboration].

hep-ex/0512070

Phys. Rev. Lett. 96, 152002 (2006)

529. "Measurement of mass and width of the excited charmed meson states D0(1) and $\mathrm{D}^{*} \mathbf{0}(2)$ at $\mathrm{CDF}$ "

A. Abulencia et al. [CDF Collaboration].

hep-ex/0512069

Phys. Rev. D 73, 051104 (2006)

530. "Search for anomalous semileptonic decay of heavy flavor hadrons produced in association with a W boson at CDF II"

A. Abulencia et al. [CDF Collaboration].

hep-ex/0512065

Phys. Rev. D 73, 051101 (2006)

531. "Measurement of the inclusive jet cross section using the $\mathrm{k}(\mathrm{t})$ algorithm in $\mathbf{p}$ anti-p collisions at $\sqrt{s}=1.96-\mathrm{TeV}$." 
A. Abulencia et al. [CDF Collaboration].

hep-ex/0512062

Phys. Rev. Lett. 96, 122001 (2006)

532. "Search for $\mathbf{H}$ to $\mathbf{b}$ anti-b produced in association with $\mathbf{W}$ bosons in $p \bar{p}$ collisions at $\sqrt{s}=1.96-\mathrm{TeV} . "$

A. Abulencia et al. [CDF Collaboration].

hep-ex/0512051

Phys. Rev. Lett. 96, 081803 (2006)

533. "Search for second-generation scalar leptoquarks in $p \bar{p}$ collisions at $\sqrt{s}=\mathbf{1 . 9 6}$ TeV."

A. Abulencia et al. [CDF Collaboration].

hep-ex/0512055

Phys. Rev. D 73, 051102 (2006)

534. "Measurement of the inclusive jet cross section in $p \bar{p}$ interactions at $\sqrt{s}=1.96$ TeV using a cone-based jet algorithm."

A. Abulencia et al. [CDF Collaboration].

hep-ex/0512020

Phys. Rev. D 74, 071103 (2006)

535. "Measurement of the top quark mass with the dynamical likelihood method using lepton plus jets events with b-tags in $p \bar{p}$ collisions at $\sqrt{s}=1.96-\mathrm{TeV}$."

A. Abulencia et al. [CDF Collaboration].

hep-ex/0512009

Phys. Rev. D 73, 092002 (2006)

536. "Measurement of the helicity of $W$ bosons in top-quark decays"

A. Abulencia et al. [CDF Collaboration].

hep-ex/0511023

Phys. Rev. D 73, 111103 (2006)

537. "Search for charged Higgs bosons from top quark decays in $p \bar{p}$ collisions at $\sqrt{s}=$ 1.96-TeV."

A. Abulencia et al. [CDF Collaboration].

hep-ex/0510065

Phys. Rev. Lett. 96, 042003 (2006)

538. "A search for $t \rightarrow t a u \nu q$ in $t \bar{t}$ production."

A. Abulencia et al. [CDF Collaboration].

hep-ex/0510063

Phys. Lett. B 639, 172 (2006)

539. "NuTeV structure function measurement"

M. Tzanov et al. [NuTeV Collaboration].

Int. J. Mod. Phys. A 20, 3759 (2005). 
540. "Precision top quark mass measurement in the lepton + jets topology in $\mathbf{p}$ anti-p collisions at $\sqrt{s}=1.96-\mathrm{TeV}$."

A. Abulencia et al. [CDF Collaboration].

hep-ex/0510049

Phys. Rev. Lett. 96, 022004 (2006)

541. "Top quark mass measurement using the template method in the lepton + jets channel at CDF II"

A. Abulencia et al. [CDF Collaboration].

hep-ex/0510048

Phys. Rev. D 73, 032003 (2006)

542. "MINERvA: High statistics neutrino scattering using a fine-grained detector" D. Naples et al. [MINERvA Collaboration].

Int. J. Mod. Phys. A 20, 3078 (2005).

543. "Direct search for Dirac magnetic monopoles in $p \bar{p}$ collisions at $\sqrt{s}=1.96 \mathrm{TeV}$ " A. Abulencia et al. [CDF Collaboration].

hep-ex/0509015

Phys. Rev. Lett. 96, 201801 (2006)

544. "Precise measurement of neutrino and anti-neutrino differential cross sections" M. Tzanov et al. [NuTeV Collaboration].

hep-ex/0509010

Phys. Rev. D 74, 012008 (2006)

545. "Search for neutral MSSM Higgs bosons decaying to tau pairs in $p \bar{p}$ collisions at $\sqrt{s}=1.96 \mathrm{TeV} "$

A. Abulencia et al. [CDF Collaboration].

hep-ex/0508051

Phys. Rev. Lett. 96, 011802 (2006)

546. "Search for $B_{s} \rightarrow \mu^{+} \mu^{-}$and $B_{d} \rightarrow \mu^{+} \mu^{-}$decays in $p \bar{p}$ collisions with CDF II"

A. Abulencia et al. [CDF Collaboration].

hep-ex/0508036

Phys. Rev. Lett. 95, 221805 (2005), [Erratum-ibid. 95, 249905 (2005)]

547. "Measurements of inclusive $\mathrm{W}$ and $\mathrm{Z}$ cross sections in $\mathrm{p}$ anti-p collisions at $\mathrm{s}^{* *}(1 / 2)=1.96-\mathrm{TeV} "$

A. Abulencia et al. [CDF Collaboration].

hep-ex/0508029

J. Phys. G G 34, 2457 (2007)

548. "Measurement of $b$ hadron masses in exclusive $J / \psi$ decays with the CDF detector"

D. Acosta et al. [CDF Collaboration].

hep-ex/0508022

Phys. Rev. Lett. 96, 202001 (2006) 
549. "Measurement of the ratios of branching fractions $B\left(B_{s}^{0} \rightarrow D_{s}^{-} \pi^{+}\right) / B\left(B^{0} \rightarrow D^{-} \pi^{+}\right)$ and $B\left(B^{+} \rightarrow \bar{D}^{0} \pi^{+}\right) / B\left(B^{0} \rightarrow D^{-} \pi^{+}\right) "$

A. Abulencia et al. [CDF Collaboration].

hep-ex/0508014

Phys. Rev. Lett. 96, 191801 (2006)

550. "A Unified model for inelastic e - N and nu - N cross-sections at all $\mathrm{Q}^{* *} \mathbf{2}$ "

A. Bodek and U. -k. Yang.

hep-ph $/ 0508007$

AIP Conf. Proc. 792, 257 (2005)

551. "Search for new high mass particles decaying to lepton pairs in $p \bar{p}$ collisions at $\sqrt{s}=1.96$ TeV"

A. Abulencia et al. [CDF Collaboration].

hep-ex/0507104

Phys. Rev. Lett. 95, 252001 (2005)

552. "Search for $\Lambda_{b} \rightarrow p \pi$ and $\Lambda_{b} \rightarrow p K$ decays in $p \bar{p}$ collisions at $\sqrt{s}=1.96 \mathbf{T e V}$ "

D. Acosta et al. [CDF Collaboration].

hep-ex/0507067

Phys. Rev. D 72, 051104 (2005)

553. "Search for $\mathbf{W}$ and $\mathbf{Z}$ bosons in the reaction $\bar{p} p \rightarrow 2$ jets $+\gamma$ at $\sqrt{s}=1.8$ TeV"

D. Acosta et al. [CDF Collaboration].

hep-ex/0507051

Phys. Rev. D 73, 012001 (2006)

554. "Search for first-generation scalar leptoquarks in $p \bar{p}$ collisions at $\sqrt{s}=1.96 \mathbf{T e V}$ " D. Acosta et al. [CDF Collaboration].

hep-ex/0506074

Phys. Rev. D 72, 051107 (2005)

555. "A search for supersymmetric Higgs bosons in the di-tau decay mode in $p \bar{p}$ collisions at $\sqrt{s}=1.8 \mathrm{TeV}$ "

D. Acosta et al. [CDF Collaboration].

hep-ex/0506042

Phys. Rev. D 72, 072004 (2005)

556. "Search for new physics using high mass tau pairs from $1.96 \mathrm{TeV} p \bar{p}$ collisions" D. Acosta et al. [CDF Collaboration].

hep-ex/0506034

Phys. Rev. Lett. 95, 131801 (2005)

557. "Measurement of the $t \bar{t}$ production cross section in $p \bar{p}$ collisions at $\sqrt{s}=1.96 \mathrm{TeV}$ using lepton plus jets events with semileptonic B decays to muons"

D. Acosta et al. [CDF Collaboration].

hep-ex/0506001

Phys. Rev. D 72, 032002 (2005) 
558. "Measurement of $B(t \rightarrow W b) / B(t \rightarrow W q)$ at the Collider Detector at Fermilab" D. Acosta et al. [CDF Collaboration].

hep-ex/0505091

Phys. Rev. Lett. 95, 102002 (2005)

559. "Evidence for the exclusive decay $B_{c}^{ \pm} \rightarrow J / \psi \pi^{ \pm}$and measurement of the mass of the $B_{c}$ meson"

A. Abulencia et al. [CDF Collaboration].

hep-ex/0505076

Phys. Rev. Lett. 96, 082002 (2006)

560. "Study of jet shapes in inclusive jet production in $p \bar{p}$ collisions at $\sqrt{s}=1.96 \mathrm{TeV}$ " D. Acosta et al. [CDF Collaboration].

hep-ex/0505013

Phys. Rev. D 71, 112002 (2005)

561. "Measurement of the cross section for $t \bar{t}$ production in $p \bar{p}$ collisions using the kinematics of lepton + jets events"

D. Acosta et al. [CDF Collaboration].

hep-ex/0504053

Phys. Rev. D 72, 052003 (2005)

562. " $K_{S}^{0}$ and $\Lambda^{0}$ production studies in $p \bar{p}$ collisions at $\sqrt{s}=1800-\mathrm{GeV}$ and $630-\mathrm{GeV}$ " D. Acosta et al. [CDF Collaboration].

hep-ex/0504048

Phys. Rev. D 72, 052001 (2005)

563. "Measurement of the azimuthal angle distribution of leptons from $W$ boson decays as a function of the $W$ transverse momentum in $p \bar{p}$ collisions at $\sqrt{s}=1.8$ TeV"

D. Acosta et al. [CDF Collaboration].

hep-ex/0504020

Phys. Rev. D 73, 052002 (2006)

564. "Search for Higgs bosons decaying into $b \bar{b}$ and produced in association with a vector boson in $p \bar{p}$ collisions at $\sqrt{s}=1.8 \mathrm{TeV} "$

D. Acosta et al. [CDF Collaboration].

hep-ex/0503039

Phys. Rev. Lett. 95, 051801 (2005)

565. "Search for long-lived doubly-charged Higgs bosons in $p \bar{p}$ collisions at $\sqrt{s}=1.96$ TeV"

D. Acosta et al. [CDF Collaboration].

hep-ex/0503004

Phys. Rev. Lett. 95, 071801 (2005)

566. "First evidence for $B_{s}^{0} \rightarrow \phi \phi$ decay and measurements of branching ratio and $A_{C P}$ for $B^{+} \rightarrow \phi K^{+}$ 
D. Acosta et al. [CDF Collaboration].

hep-ex/0502044

Phys. Rev. Lett. 95, 031801 (2005)

567. "Measurement of the moments of the hadronic invariant mass distribution in semileptonic $B$ decays"

D. Acosta et al. [CDF Collaboration].

hep-ex/0502003

Phys. Rev. D 71, 051103 (2005)

568. "Measurement of the $W^{+} W^{-}$production cross section in $p \bar{p}$ collisions at $\sqrt{s}=1.96$ TeV using dilepton events"

D. Acosta et al. [CDF Collaboration].

hep-ex/0501050

Phys. Rev. Lett. 94, 211801 (2005)

569. "The structure of the nucleon, three decades of investigation (1967-2004)"

A. Bodek.

Nucl. Phys. Proc. Suppl. 139, 165 (2005).

570. "Measurement of the forward-backward charge asymmetry from $W \rightarrow e \nu$ production in $p \bar{p}$ collisions at $\sqrt{s}=1.96 \mathbf{T e V}^{\prime \prime}$

D. Acosta et al. [CDF Collaboration].

hep-ex/0501023

Phys. Rev. D 71, 051104 (2005)

571. "Search for $Z Z$ and $Z W$ production in $p \bar{p}$ collisions at $\sqrt{s}=1.96 \mathbf{T e V}$ "

D. Acosta et al. [CDF Collaboration].

hep-ex/0501021

Phys. Rev. D 71, 091105 (2005)

572. "Unified approach for modelling neutrino and electron nucleon scattering cross sections from very high $\mathrm{Q}^{* *} 2$ to $\mathrm{Q}^{* *} 2=0$ "

A. Bodek and U. K. Yang.

AIP Conf. Proc. 721, 358 (2004).

573. "Measurement of the $J / \psi$ meson and $b$-hadron production cross sections in $p \bar{p}$ collisions at $\sqrt{s}=1960 \mathrm{GeV}^{\prime \prime}$

D. Acosta et al. [CDF Collaboration]. hep-ex/0412071

Phys. Rev. D 71, 032001 (2005)

574. "Measurement of the lifetime difference between B(s) mass eigenstates"

D. Acosta et al. [CDF Collaboration].

hep-ex/0412057

Phys. Rev. Lett. 94, 101803 (2005) 
575. "Measurement of the cross section for prompt diphoton production in $p \bar{p}$ collisions at $\sqrt{s}=1.96 \mathrm{TeV}^{\prime \prime}$

D. Acosta et al. [CDF Collaboration].

hep-ex/0412050

Phys. Rev. Lett. 95, 022003 (2005)

576. "Search for anomalous kinematics in $t \bar{t}$ dilepton events at CDF II"

D. Acosta et al. [CDF Collaboration].

hep-ex/0412042

Phys. Rev. Lett. 95, 022001 (2005)

577. "Measurements of $b \bar{b}$ azimuthal production correlations in $p \bar{p}$ collisions at $\sqrt{s}=1.8$ TeV"

D. Acosta et al. [CDF Collaboration].

hep-ex/0412006

Phys. Rev. D 71, 092001 (2005)

578. "Measurement of the $W$ boson polarization in top decay at CDF at $\sqrt{s}=1.8$ TeV"

D. Acosta et al. [CDF Collaboration].

hep-ex/0411070

Phys. Rev. D 71, 031101 (2005), [Erratum-ibid. D 71, 059901 (2005)]

579. "Measurement of the forward-backward charge asymmetry of electron positron pairs in $p \bar{p}$ collisions at $\sqrt{s}=1.96 \mathrm{TeV}^{\prime \prime}$

D. Acosta et al. [CDF Collaboration].

hep-ex/0411059

Phys. Rev. D 71, 052002 (2005)

580. "Improved low $\mathrm{Q}^{* *} 2$ model for neutrino and electron nucleon cross sections in few GeV region"

A. Bodek, I. Park and U. -k. Yang.

hep-ph/0411202

Nucl. Phys. Proc. Suppl. 139, 113 (2005)

581. "The JUPITER electron scattering program at Jefferson Lab"

A. Bodek. hep-ex/0411044

Int. J. Mod. Phys. A 20, 3089 (2005)

582. "Measurement of charged particle multiplicities in gluon and quark jets in $p \bar{p}$ collisions at $\sqrt{s}=1.8 \mathrm{TeV}^{\prime \prime}$

D. Acosta et al. [CDF Collaboration].

Phys. Rev. Lett. 94, 171802 (2005).

583. "Search for scalar leptoquark pairs decaying to $\nu \bar{\nu} q \bar{q}$ in $p \bar{p}$ collisions at $\sqrt{s}=1.96$ TeV"

D. Acosta et al. [CDF Collaboration]. 
hep-ex/0410076

Phys. Rev. D 71, 112001 (2005), [Erratum-ibid. D 71, 119901 (2005)]

584. "Search for electroweak single top quark production in $p \bar{p}$ collisions at $\sqrt{s}=1.96$ TeV"

D. Acosta et al. [CDF Collaboration].

hep-ex/0410058

Phys. Rev. D 71, 012005 (2005)

585. "Vector and axial form-factors applied to neutrino quasielastic scattering"

H. S. Budd, A. Bodek and J. Arrington.

hep-ex/0410055

Nucl. Phys. Proc. Suppl. 139, 90 (2005)

586. "Search for anomalous production of diphoton events with missing transverse energy at CDF and limits on gauge-mediated supersymmetry-breaking models" D. Acosta et al. [CDF Collaboration].

hep-ex/0410053

Phys. Rev. D 71, 031104 (2005)

587. "Measurement of the $t \bar{t}$ production cross section in $p \bar{p}$ collisions at $\sqrt{s}=1.96 \mathrm{TeV}$ using lepton + jets events with secondary vertex $b$-tagging"

D. Acosta et al. [CDF Collaboration].

hep-ex/0410041

Phys. Rev. D 71, 052003 (2005)

588. "Comparison of three-jet events in $p \bar{p}$ collisions at $\sqrt{s}=1.8 \mathrm{TeV}$ to predictions from a next-to-leading order QCD calculation"

D. Acosta et al. [CDF Collaboration].

hep-ex/0410018

Phys. Rev. D 71, 032002 (2005)

589. "Measurement of partial widths and search for direct CP violation in $D^{0}$ meson decays to $K^{-} K^{+}$and $\pi^{-} \pi^{+}$"

D. Acosta et al. [CDF Collaboration].

hep-ex/0504006

Phys. Rev. Lett. 94, 122001 (2005)

590. "Search for excited and exotic electrons in the $e \gamma$ decay channel in $p \bar{p}$ collisions at $\sqrt{s}=1.96 \mathrm{TeV}^{\prime \prime}$

D. Acosta et al. [CDF Collaboration].

hep-ex/0410013

Phys. Rev. Lett. 94, 101802 (2005)

591. "Measurement of $W \gamma$ and $Z \gamma$ production in $p \bar{p}$ collisions at $\sqrt{s}=1.96 \mathbf{T e V}$ "

D. Acosta et al. [CDF Collaboration].

hep-ex/0410008

Phys. Rev. Lett. 94, 041803 (2005) 
592. "Neutrino scattering uncertainties and their role in long baseline oscillation experiments"

D. A. Harris et al. [MINERvA Collaboration].

hep-ex/0410005

593. "Measurement of the $t \bar{t}$ production cross section in $p \bar{p}$ collisions at $\sqrt{s}=1.96 \mathrm{TeV}$ using kinematic fitting of $b$-tagged lepton + jet events"

D. Acosta et al. [CDF Collaboration].

hep-ex/0409029

Phys. Rev. D 71, 072005 (2005)

594. "Cross section measurements and charm production in the NuTeV experiment" S. Boyd et al. [NuTeV Collaboration].

AIP Conf. Proc. 698, 95 (2004).

595. "First measurements of inclusive $W$ and $Z$ cross sections from Run II of the Tevatron collider"

D. Acosta et al. [CDF Collaboration].

hep-ex/0406078

Phys. Rev. Lett. 94, 091803 (2005)

596. "Search for doubly-charged Higgs bosons decaying to dileptons in $p \bar{p}$ collisions at $\sqrt{s}=1.96$ TeV"

D. Acosta et al. [CDF Collaboration].

hep-ex/0406073

Phys. Rev. Lett. 93, 221802 (2004)

597. "Inclusive search for anomalous production of high $p_{T}$ like-sign lepton pairs in $p \bar{p}$ collisions at $\sqrt{s}=1.8 \mathrm{TeV}^{\prime \prime}$

D. Acosta et al. [CDF Collaboration].

hep-ex/0405063

Phys. Rev. Lett. 93, 061802 (2004)

598. "Proposal to perform a high-statistics neutrino scattering experiment using a fine-grained detector in the NuMI beam"

D. Drakoulakos et al. [Minerva Collaboration].

hep-ex/0405002

599. "Measurement of the $t \bar{t}$ production cross section in $p \bar{p}$ collisions at $\sqrt{s}=1.96 \mathrm{TeV}$ using dilepton events"

D. Acosta et al. [CDF Collaboration].

hep-ex/0404036

Phys. Rev. Lett. 93, 142001 (2004)

600. "Direct photon cross section with conversions at CDF"

D. Acosta et al. [CDF Collaboration].

hep-ex/0404022

Phys. Rev. D 70, 074008 (2004) 
601. "The underlying event in hard interactions at the Tevatron $\bar{p} p$ collider" D. Acosta et al. [CDF Collaboration].

hep-ex/0404004

Phys. Rev. D 70, 072002 (2004)

602. "Optimized search for single top quark production at the Fermilab tevatron" D. Acosta et al. [CDF Collaboration].

Phys. Rev. D 69, 052003 (2004).

603. "Search for $B_{s}^{0} \rightarrow \mu^{+} \mu^{-}$and $B_{d}^{0} \rightarrow \mu^{+} \mu^{-}$decays in $p \bar{p}$ collisions at $\sqrt{s}=1.96$ TeV" D. Acousta et al. [CDF Collaboration].

hep-ex/0403032

Phys. Rev. Lett. 93, 032001 (2004)

604. "Unified approach for modelling neutrino and electron nucleon scattering cross sections from high energy to very low energy"

A. Bodek and U. K. Yang.

605. " $\sin ^{2} \theta_{W}$ from neutrino scattering at NUTEV"

K. S. McFarland, G. P. Zeller, T. Adams, A. Alton, S. Avvakumov, L. de Barbaro, P. de Barbaro and R. H. Bernstein et al..

Int. J. Mod. Phys. A 18, 3841 (2003).

606. "Observation of the narrow state $X(3872) \rightarrow J / \psi \pi^{+} \pi^{-}$in $\bar{p} p$ collisions at $\sqrt{s}=1.96$ TeV"

D. Acosta et al. [CDF Collaboration].

hep-ex/0312021

Phys. Rev. Lett. 93, 072001 (2004)

607. "High energy neutrino scattering results from NuTeV"

D. Naples, T. Adams, A. Alton, S. Avvakumov, L. de Barbaro, P. de Barbaro, R. H. Bernstein and A. Bodek et al..

Nucl. Phys. Proc. Suppl. 118, 164 (2003).

608. "Measurement of the polar-angle distribution of leptons from $W$ boson decay as a function of the $\mathbf{W}$ transverse momentum in $p \bar{p}$ collisions at $\sqrt{s}=1.8 \mathrm{TeV}$ " D. Acosta et al. [CDF Collaboration]. hep-ex/0311050

Phys. Rev. D 70, 032004 (2004)

609. "Combination of CDF and DØ results on $W$ boson mass and width" V. M. Abazov et al. [CDF and D0 Collaboration]. hep-ex/0311039

Phys. Rev. D 70, 092008 (2004)

610. "Inclusive double pomeron exchange at the Fermilab Tevatron $\bar{p} p$ collider" D. Acosta et al. [CDF Collaboration].

hep-ex/0311023

Phys. Rev. Lett. 93, 141601 (2004) 
611. "Search for Kaluza-Klein graviton emission in $p \bar{p}$ collisions at $\sqrt{s}=1.8$-TeV using the missing energy signature"

D. Acosta et al. [CDF Collaboration].

hep-ex/0309051

Phys. Rev. Lett. 92, 121802 (2004)

612. "Measurement of the average time-integrated mixing probability of $b$-flavored hadrons produced at the Tevatron"

D. Acosta et al. [CDF Collaboration].

hep-ex/0309030

Phys. Rev. D 69, 012002 (2004)

613. "Modeling neutrino quasielastic cross-sections on nucleons and nuclei"

A. Bodek, H. S. Budd and J. Arrington.

hep-ex/0309024

AIP Conf. Proc. 698, 148 (2004)

614. "Search for the flavor-changing neutral current decay $D^{0} \rightarrow \mu^{+} \mu^{-}$in $p \bar{p}$ collisions at $\sqrt{s}=1.96 \mathbf{T e V}^{\prime}$

D. Acosta et al. [CDF Collaboration].

hep-ex/0308059

Phys. Rev. D 68, 091101 (2003)

615. "Measurement of the mass difference $m\left(D_{s}^{+}\right)-m\left(D^{+}\right)$at CDF II"

D. Acosta et al. [CDF Collaboration].

hep-ex/0310043

Phys. Rev. D 68, 072004 (2003)

616. "Modeling neutrino and electron scattering inelastic cross- sections in the few GeV region with effective LO PDFs TV Leading Order"

A. Bodek and U. K. Yang.

hep-ex/0308007

617. "Modeling quasielastic form-factors for electron and neutrino scattering" H. S. Budd, A. Bodek and J. Arrington.

hep-ex/0308005

618. "Measurement of prompt charm meson production cross sections in $p \bar{p}$ collisions at $\sqrt{s}=1.96 \mathrm{TeV}^{\prime}$

D. Acosta et al. [CDF Collaboration].

hep-ex/0307080

Phys. Rev. Lett. 91, 241804 (2003)

619. "Search for associated production of $\Upsilon$ and vector boson in $p \bar{p}$ collisions at $\sqrt{s}=1.8$ TeV"

D. Acosta et al. [CDF Collaboration].

Phys. Rev. Lett. 90, 221803 (2003). 
620. "Search for lepton flavor violating decays of a heavy neutral particle in $p \bar{p}$ collisions at $\sqrt{s}=1.8 \mathrm{TeV}^{\prime \prime}$

D. Acosta et al. [CDF Collaboration].

hep-ex/0307012

Phys. Rev. Lett. 91, 171602 (2003)

621. "NuTeV cross-section and structure function measurements"

D. Naples et al. [NuTeV Collaboration].

hep-ex/0307005

622. "New QCD results from NuTeV"

M. Tzanov et al. [NuTeV Collaboration].

hep-ex/0306035

623. "Search for pair production of scalar top quarks in $R$-parity violating decay modes in $p \bar{p}$ collisions at $\sqrt{s}=1.8 \mathrm{TeV}^{\prime \prime}$

D. Acosta et al. [CDF Collaboration].

hep-ex/0305010

Phys. Rev. Lett. 92, 051803 (2004)

624. "Central pseudorapidity gaps in events with a leading antiproton at the Fermilab Tevatron $\bar{p} p$ collider"

D. Acosta et al. [CDF Collaboration].

hep-ex/0303011

Phys. Rev. Lett. 91, 011802 (2003)

625. "Search for the supersymmetric partner of the top quark in dilepton events from $p \bar{p}$ collisions at $\sqrt{s}=1.8 \mathrm{TeV}^{\prime \prime}$

D. Acosta et al. [CDF Collaboration].

hep-ex/0302009

Phys. Rev. Lett. 90, 251801 (2003)

626. "Modeling neutrino and electron scattering cross-sections in the few GeV region with effective LO PDFs"

A. Bodek and U. K. Yang.

hep-ex/0301036

AIP Conf. Proc. 670, 110 (2003)

627. "Detector $\mathbf{R}$ and $\mathbf{D}$ for future neutrino experiments with the NuMI beamline" G. Barenboim, A. Bodek, C. Bromberg, A. Bross, L. Buckley-Geer, B. Choudhary, D. Cline and F. DeJongh et al..

hep-ex/0304017

628. "Nuclear effects and the NuTeV $\sin * * 2(\operatorname{theta}(\mathrm{W}))$ measurement"

K. S. McFarland, G. P. Zeller, T. Adams, A. Alton, S. Avvakumov, L. de Barbaro, P. de Barbaro and R. H. Bernstein et al.

Nucl. Phys. Proc. Suppl. 112, 226 (2002). 
629. "Cross section for forward $J / \psi$ production in $p \bar{p}$ collisions at $\sqrt{s}=1.8$ TeV" D. Acosta et al. [CDF Collaboration].

Phys. Rev. D 66, 092001 (2002).

630. "Search for long-lived charged massive particles in $\bar{p} p$ collisions at $\sqrt{s}=1.8$ TeV" D. Acosta et al. [CDF Collaboration].

hep-ex/0211064

Phys. Rev. Lett. 90, 131801 (2003)

631. "Intercalibration of the longitudinal segments of a calorimeter system"

M. G. Albrow, S. Aota, G. Apollinari, T. Asakawa, M. Bailey, P. de Barbaro, V. Barnes and D. Benjamin et al..

Nucl. Instrum. Meth. A 487, 381 (2002).

632. "Higher twist, xi(omega) scaling, and effective LO PDFs for lepton scattering in the few GeV region"

ABodek and U. K. Yang.

hep-ex/0210024

J. Phys. G G 29, 1899 (2003)

633. "Off the mass shell: Electroweak physics at NuTeV"

K. S. McFarland et al. [NuTeV Collaboration].

hep-ex/0210010

eConf C 020620, SABT01 (2002)

634. "Search for a $W^{\prime}$ boson decaying to a top and bottom quark pair in $1.8 \mathrm{TeV} p \bar{p}$ collisions"

D. Acosta et al. [CDF Collaboration].

hep-ex/0209030

Phys. Rev. Lett. 90, 081802 (2003)

635. "Search for radiative b-hadron decays in $p \bar{p}$ collisions at $\sqrt{s}=1.8 \mathrm{TeV}$ "

D. Acosta et al. [CDF Collaboration].

hep-ex/0208035

Phys. Rev. D 66, 112002 (2002)

636. "Reply to the comment on 'A Precise determination of electroweak parameters in neutrino nucleon scattering",

G. P. Zeller et al. [NuTeV Collaboration].

hep-ex/0207052

637. "Momentum distribution of charged particles in jets in dijet events in $p \bar{p}$ collisions at $\sqrt{s}=1.8 \mathrm{TeV}$ and comparisons to perturbative QCD predictions"

D. Acosta et al. [CDF Collaboration].

Phys. Rev. D 68, 012003 (2003). 
638. "Measurement of the ratio of $b$ quark production cross sections in $\bar{p} p$ collisions at $\sqrt{s}=630 \mathrm{GeV}$ and $\sqrt{s}=1800 \mathbf{G e V}^{\prime \prime}$

D. Acosta et al. [CDF Collaboration].

hep-ex/0206019

Phys. Rev. D 66, 032002 (2002)

639. "Branching ratio measurements of exclusive $B^{+}$decays to charmonium with the Collider Detector at Fermilab"

D. Acosta et al. [CDF Collaboration].

Phys. Rev. D 66, 052005 (2002).

640. "A Departure from prediction: Electroweak physics at NuTeV"

K. S. McFarland, G. P. Zeller, T. Adams, A. Alton, S. Avvakumov, L. de Barbaro, P. de Barbaro and R. H. Bernstein et al..

hep-ex/0205080

641. "Limits on extra dimensions and new particle production in the exclusive photon and missing energy signature in $p \bar{p}$ collisions at $\sqrt{s}=1.8 \mathrm{TeV}$ "

D. Acosta et al. [CDF Collaboration].

hep-ex/0205057

Phys. Rev. Lett. 89, 281801 (2002)

642. "Measurement of $B$ meson lifetimes using fully reconstructed $B$ decays produced in $p \bar{p}$ collisions at $\sqrt{s}=1.8 \mathrm{TeV}$ "

D. Acosta et al. [CDF Collaboration].

Phys. Rev. D 65, 092009 (2002).

643. "A Search for muon-neutrino - i electron-neutrino and muon-anti-neutrino - $i$ electron-anti-neutrino oscillations at NuTeV"

S. Avvakumov, T. Adams, A. Alton, L. de Barbaro, P. de Barbaro, R. H. Bernstein, A. Bodek and T. Bolton et al..

hep-ex/0203018

Phys. Rev. Lett. 89, 011804 (2002)

644. "Modeling deep inelastic cross-sections in the few GeV region"

A. Bodek and U. K. Yang.

hep-ex/0203009

Nucl. Phys. Proc. Suppl. 112, 70 (2002)

645. "On the effect of asymmetric strange seas and isospin violating parton distribution functions on $\sin ^{2} \theta_{W}$ measured in the NuTeV experiment"

G. P. Zeller et al. [NuTeV Collaboration].

hep-ex/0203004

Phys. Rev. D 65, 111103 (2002), [Erratum-ibid. D 67, 119902 (2003)]

646. "Search for new physics in photon lepton events in $p \bar{p}$ collisions at $\sqrt{s}=1.8-\mathrm{TeV}$ " D. Acosta et al. [CDF Collaboration]. 
hep-ex/0202044

Phys. Rev. Lett. 89, 041802 (2002)

647. "Comparison of the isolated direct photon cross sections in $p \bar{p}$ collisions at $\sqrt{s}=$ 1.8-TeV and $\sqrt{s}=0.63-\mathrm{TeV}^{\prime}$

D. Acosta et al. [CDF Collaboration].

hep-ex/0201004

Phys. Rev. D 65, 112003 (2002)

648. "Observation of neutral current charm production in $\nu_{m} u$ Fe scattering at the Fermilab Tevatron"

A. Alton et al. [NuTeV Collaboration].

Int. J. Mod. Phys. A 16S1B, 764 (2001).

649. "Differential cross-section results from NuTeV"

D. Naples, J. McDonald, V. Radescu, M. Tzanov, T. Adams, A. Alton, T. Bolton and J. Goldman et al.

650. "Search for light-to-heavy quark flavor changing neutral currents in $\mathbf{n u} / \mathbf{m u ~} \mathbf{N}$ and anti-nu/mu N scattering"

A. Alton et al. [NuTeV Collaboration].

Int. J. Mod. Phys. A 16S1B, 489 (2001).

651. " $\Upsilon$ production and polarization in $p \bar{p}$ collisions at $\sqrt{s}=1.8-\mathrm{TeV}^{\prime}$

D. Acosta et al. [CDF Collaboration].

Phys. Rev. Lett. 88, 161802 (2002).

652. "Soft and hard interactions in $p \bar{p}$ collisions at $\sqrt{s}=1800-\mathrm{GeV}$ and $630-\mathrm{GeV}$ "

D. Acosta et al. [CDF Collaboration].

Phys. Rev. D 65, 072005 (2002).

653. "Measurement of the $B^{+}$total cross section and $B^{+}$differential cross section $d \sigma / d p_{T}$ in $p \bar{p}$ collisions at $\sqrt{s}=\mathbf{1 . 8 - \mathbf { T e V }}$ "

D. Acosta et al. [CDF Collaboration].

hep-ph/0111359

Phys. Rev. D 65, 052005 (2002)

654. "Search for single top quark production in $p \bar{p}$ collisions at $\sqrt{s}=1.8-\mathrm{TeV}$ "

D. Acosta et al. [CDF Collaboration].

hep-ex/0110067

Phys. Rev. D 65, 091102 (2002)

655. "A Precise determination of electroweak parameters in neutrino nucleon scattering"

G. P. Zeller et al. [NuTeV Collaboration].

hep-ex/0110059

Phys. Rev. Lett. 88, 091802 (2002), [Erratum-ibid. 90, 239902 (2003)] 
656. "Search for the decay $B_{s} \rightarrow \mu^{+} \mu^{-} \phi$ in $p \bar{p}$ collisions at $\sqrt{s}=1.8-\mathbf{T e V}$ " D. Acosta et al. [CDF Collaboration].

Phys. Rev. D 65, 111101 (2002).

657. "Charged jet evolution and the underlying event in $p \bar{p}$ collisions at $1.8 \mathrm{TeV}$ " T. Affolder et al. [CDF Collaboration].

Phys. Rev. D 65, 092002 (2002).

658. "Search for new physics in photon lepton events in $\mathrm{p}$ anti-p collisions at $\mathrm{s}^{* *}(1 / 2)$ $=1.8-\mathrm{TeV}^{\prime}$

D. Acosta et al. [CDF Collaboration]. hep-ex/0110015

Phys. Rev. D 66, 012004 (2002)

659. "Diffractive dijet production at $\sqrt{s}=630 \mathrm{GeV}$ and $\mathbf{1 8 0 0} \mathbf{~ G e V}$ at the Fermilab Tevatron"

D. Acosta et al. [CDF Collaboration].

hep-ex/0109025

Phys. Rev. Lett. 88, 151802 (2002)

660. "Study of the heavy flavor content of jets produced in association with W bosons in $p \bar{p}$ collisions at $\sqrt{s}=1.8 \mathrm{TeV}$ "

D. Acosta et al. [CDF Collaboration]. hep-ex/0109012

Phys. Rev. D 65, 052007 (2002)

661. "Measurement of the strong coupling constant from inclusive jet production at the Tevatron $\bar{p} p$ collider"

T. Affolder et al. [CDF Collaboration].

hep-ex/0108034

Phys. Rev. Lett. 88, 042001 (2002)

662. "A study of $B^{0} \rightarrow J / \psi K^{(*) 0} \pi^{+} \pi^{-}$decays with the Collider Detector at Fermilab" T. Affolder et al. [CDF Collaboration].

hep-ex/0108022

Phys. Rev. Lett. 88, 071801 (2002)

663. "Search for new heavy particles in the $W Z^{0}$ final state in $p \bar{p}$ collisions at $\sqrt{s}=$ $1.8 \mathrm{TeV}$ "

T. Affolder et al. [CDF Collaboration].

hep-ex/0108004

Phys. Rev. Lett. 88, 071806 (2002)

664. "Preliminary measurement of the differential cross-section from neutrino nucleon deeply inelastic scattering at NuTeV"

J. McDonald et al. [NuTeV Collaboration].

hep-ex/0107082 
665. "Observation of diffractive $J / \psi$ production at the Fermilab Tevatron" T. Affolder et al. [CDF Collaboration].

hep-ex/0107071

Phys. Rev. Lett. 87, 241802 (2001)

666. "Charged particle multiplicity in jets in $p \bar{p}$ collisions at $\sqrt{s}=1.8 \mathrm{TeV}$ " T. Affolder et al. [CDF Collaboration].

Phys. Rev. Lett. 87, 211804 (2001).

667. "The CDF plug upgrade electromagnetic calorimeter: Test beam results" M. G. Albrow et al. [CDF Collaboration].

Nucl. Instrum. Meth. A 480, 524 (2002).

668. "Search for quark lepton compositeness and a heavy $W^{\prime}$ boson using the $e \nu$ channel in $p \bar{p}$ collisions at $\sqrt{s}=1.8 \mathrm{TeV}$ "

T. Affolder et al. [CDF Collaboration].

hep-ex/0107008

Phys. Rev. Lett. 87, 231803 (2001)

669. "Searches for new physics in events with a photon and b-quark jet at CDF" T. Affolder et al. [CDF Collaboration].

hep-ex/0106012

Phys. Rev. D 65, 052006 (2002)

670. "Search for gluinos and squarks using like-sign dileptons in $p \bar{p}$ collisions at $\sqrt{s}=1.8$ TeV"

T. Affolder et al. [CDF Collaboration].

hep-ex/0106061

Phys. Rev. Lett. 87, 251803 (2001)

671. "Measurement of $d(\sigma) / d M$ and forward-backward charge asymmetry for high mass Drell-Yan $e^{+} e^{-}$pairs from $p \bar{p}$ collisions at $\sqrt{s}=1.8 \mathbf{T e V}^{\prime \prime}$

T. Affolder et al. [CDF Collaboration].

hep-ex/0106047

Phys. Rev. Lett. 87, 131802 (2001)

672. "Cross section and heavy quark composition of $\gamma+\mu$ events produced in $p \bar{p}$ collisions"

T. Affolder et al. [CDF Collaboration].

hep-ex/0106004

Phys. Rev. D 65, 012003 (2002)

673. "Search for gluinos and scalar quarks in $p \bar{p}$ collisions at $\sqrt{s}=1.8$ TeV using the missing energy plus multijets signature"

T. Affolder et al. [CDF Collaboration].

hep-ex/0106001

Phys. Rev. Lett. 88, 041801 (2002) 
674. "Production of $\chi_{c 1}$ and $\chi_{c 2}$ in $p \bar{p}$ collisions at $\sqrt{s}=1.8 \mathrm{TeV}$ "

T. Affolder et al. [CDF Collaboration].

Phys. Rev. Lett. 86, 3963 (2001).

675. "Double diffraction dissociation at the Fermilab Tevatron collider"

T. Affolder et al. [CDF Collaboration].

hep-ex/0107070

Phys. Rev. Lett. 87, 141802 (2001)

676. "Extraction of $\mathbf{R}=\operatorname{sigma}(\mathrm{L}) / \operatorname{sigma}(\mathrm{T})$ from CCRF Fe neutrino(muon) and Fe anti-neutrino(muon) differential cross-sections"

A. Bodek et al. [CCFR/NuTeV Collaboration].

hep-ex/0105067

677. "Search for narrow diphoton resonances and for $\gamma \gamma+W / Z$ signatures in $p \bar{p}$ collisions at $\sqrt{s}=1.8 \mathrm{TeV}^{\prime \prime}$

T. Affolder et al. [CDF Collaboration].

hep-ex/0105066

Phys. Rev. D 64, 092002 (2001)

678. "Physics Potential and Feasibility of UNO"

M. Goodman, M. Goldhaber, M. Diwan, B. Viren, D. Ferenc, S. Barwick, D. Casper and

W. Gajewski et al..

679. "Extraction of $\mathbf{R}=\operatorname{sigma}(\mathbf{L}) / \operatorname{sigma}(\mathbf{T})$ from CCFR Fe-neutrino(muon) and Fe-anti-neutrino(muon) differential cross-sections"

U. -K. Yang et al. [CCFR/NuTeV Collaboration].

hep-ex/0104040

Phys. Rev. Lett. 87, 251802 (2001)

680. "Observation of an anomalous number of dimuon events in a high-energy neutrino beam"

T. Adams et al. [NuTeV Collaboration].

hep-ex/0104037

Phys. Rev. Lett. 87, 041801 (2001)

681. "Search for the lepton number violating process anti-neutrino(muon) e- -i muon- anti-neutrino(e)"

J. A. Formaggio et al. [NuTeV Collaboration].

hep-ex/0104029

Phys. Rev. Lett. 87, 071803 (2001)

682. "Precise measurement of dimuon production cross-sections in muon neutrino Fe and muon anti-neutrino Fe deep inelastic scattering at the Tevatron"

M. Goncharov et al. [NuTeV Collaboration].

hep-ex/0102049

Phys. Rev. D 64, 112006 (2001) 
683. "Implications of a $300-\mathrm{GeV} / \mathrm{c}$ to $500-\mathrm{Gev} / \mathrm{c} Z^{\prime}$ boson on $p$ antip collider data at $S^{(1 / 2)}=1.8-\mathrm{TeV}^{\prime \prime}$

A. Bodek and U. Baur.

hep-ph/0102160

Eur. Phys. J. C 21, 607 (2001)

684. "Measurement of the inclusive jet cross section in $\bar{p} p$ collisions at $\sqrt{s}=1.8 \mathrm{TeV}$ " T. Affolder et al. [CDF Collaboration].

hep-ph/0102074

Phys. Rev. D 64, 032001 (2001), [Erratum-ibid. D 65, 039903 (2002)]

685. "Measurement of the $t \bar{t}$ production cross section in $p \bar{p}$ collisions at $\sqrt{s}=1.8 \mathrm{TeV}$ " T. Affolder et al. [CDF Collaboration].

hep-ex/0101036

Phys. Rev. D 64, 032002 (2001), [Erratum-ibid. D 67, 119901 (2003)]

686. "Measuring the strange sea at NuTeV"

T. Adams, A. Alton, S. Avvakumov, L. de Barbaro, P. de Barbaro, R. H. Bernstein, A. Bodek and T. Bolton et al..

687. "First measurement of the ratio $B(t \rightarrow W b) / B(t \rightarrow W q)$ and associated limit on the CKM element $\left|V_{t b}\right|$ "

T. Affolder et al. [CDF Collaboration].

hep-ex/0012029

Phys. Rev. Lett. 86, 3233 (2001)

688. "Measurement of the two-jet differential cross section in $p \bar{p}$ collisions at $\sqrt{s}=1800$ GeV"

T. Affolder et al. [CDF Collaboration].

hep-ex/0012013

Phys. Rev. D 64, 012001 (2001), [Erratum-ibid. D 65, 039902 (2002)]

689. "Recent structure function results from CCFR"

B. T. Fleming et al. [CCFR and NuTeV Collaborations].

hep-ex/0011095

690. "A First measurement of low $\mathrm{x}$ low $\mathrm{Q}^{* *} 2$ structure functions in neutrino scattering"

B. T. Fleming et al. [CCFR and NuTeV Collaborations].

hep-ex/0011094

Phys. Rev. Lett. 86, 5430 (2001)

691. "Measurement of the top quark $p_{T}$ distribution"

T. Affolder et al. [CDF Collaboration].

Phys. Rev. Lett. 87, 102001 (2001).

692. "Search for the supersymmetric partner of the top quark in $p \bar{p}$ collisions at $\sqrt{s}=$ 1.8 TeV" 
T. Affolder et al. [CDF Collaboration].

hep-ex/0011004

Phys. Rev. D 63, 091101 (2001)

693. "Search for neutral supersymmetric Higgs bosons in $p \bar{p}$ collisions at $\sqrt{s}=1.8$ TeV"

T. Affolder et al. [CDF Collaboration].

hep-ex/0010052

Phys. Rev. Lett. 86, 4472 (2001)

694. "Recent structure function results from neutrino scattering at Fermilab"

U. -K. Yang et al. [CCFR / NuTeV Collaboration].

hep-ex/0010001

695. "Measurement of d sigma / d y for high mass drell-yan e+ e- pairs at CDF"

A. Bodek [CDF Collaboration].

hep-ex/0009067

Int. J. Mod. Phys. A 16S1A, 262 (2001)

696. "New measurements of nucleon structure functions from CCRF / NuTeV"

A. Bodek et al. [CCRF/NuTeV Collaboration].

hep-ex/0009061

Int. J. Mod. Phys. A 16S1A, 202 (2001)

697. "Test of enhanced leading order QCD in $W$ boson plus jets events from $1.8 \mathrm{TeV}$ $\bar{p} p$ collisions"

T. Affolder et al. [CDF Collaboration].

Phys. Rev. D 63, 072003 (2001).

698. "Measurements of $F_{2}$ and $x F_{3}^{\nu}-x F_{3}^{\bar{\nu}}$ from CCFR $\nu_{\mu}-$ Fe and $\bar{\nu}_{\mu}-$ Fe data in a physics model independent way"

U. -K. Yang et al. [CCFR/NuTeV Collaboration].

hep-ex/0009041

Phys. Rev. Lett. 86, 2742 (2001)

699. "Observation of anomalous dimuon events in the NuTeV decay detector: Preliminary"

T. Adams et al. [NuTeV Collaboration].

hep-ex/0009007

Int. J. Mod. Phys. A 16S1B, 761 (2001)

700. "Observation of neutral current charm production in muon neutrino Fe scattering at the Tevatron"

A. Alton et al. [NuTeV Collaboration].

hep-ex/0008068

Phys. Rev. D 64, 012002 (2001) 
701. "Physics at a neutrino factory"

C. Albright, G. Anderson, V. Barger, R. Bernstein, G. Blazey, A. Bodek, E. Buckley-Geer and A. Bueno et al..

hep-ex/0008064

702. "New measurements of nucleon structure functions from the CCFR/NuTeV Collaboration"

A. Bodek et al. [CCFR / NuTeV Collaboration].

hep-ex/0008003

AIP Conf. Proc. 549, 509 (2000)

703. "Search for light to heavy quark flavor changing neutral currents in $\nu_{\mu} N$ and anti-muon-neutrino $N$ scattering at the Tevatron"

A. Alton, T. Adams, T. Bolton, J. Goldman, M. Goncharov, D. Naples, R. A. Johnson and M. Vakili et al..

hep-ex/0007059

Phys. Rev. D 63, 012001 (2001)

704. "Measurement of the $W$ boson mass with the Collider Detector at Fermilab"

T. Affolder et al. [CDF Collaboration].

hep-ex/0007044

Phys. Rev. D 64, 052001 (2001)

705. "Measurement of the decay amplitudes of $B^{0} \rightarrow J / \psi K^{* 0}$ and $B_{s}^{0} \rightarrow J / \psi \phi$ decays" T. Affolder et al. [CDF Collaboration].

hep-ex/0007034

Phys. Rev. Lett. 85, 4668 (2000)

706. "Recent QCD results from NuTeV / CCFR Collaboration"

T. Adams et al. [NuTeV/CCFR Collaboration].

Nucl. Phys. Proc. Suppl. 86, 93 (2000).

707. "A Feasibility study of a neutrino source based on a muon storage ring" N. Holtkamp, (ed.), D. A. Finley, (ed.), T. Anderson, N. Andreev, C. M. Ankenbrandt, S. Assadi, M. Atac and C. Bhat et al..

708. "Measurement of the top quark mass with the Collider Detector at Fermilab" T. Affolder et al. [CDF Collaboration].

hep-ex/0006028

Phys. Rev. D 63, 032003 (2001)

709. "Measurement of $d(\sigma) / d y$ for high mass Drell-Yan $e^{+} e^{-}$pairs from $p \bar{p}$ collisions at $\sqrt{s}=1.8 \mathrm{TeV} "$

T. Affolder et al. [CDF Collaboration].

hep-ex/0006025

Phys. Rev. D 63, 011101 (2001) 
710. "Dijet production by double pomeron exchange at the Fermilab Tevatron" T. Affolder et al. [CDF Collaboration].

Phys. Rev. Lett. 85, 4215 (2000).

711. "Measurements of $\mathbf{F}(2), \times \mathbf{F}^{* *}$ neutrino(3) - $\times \mathbf{F}^{* *}$ anti-neutrino(3) from CCFR Fe-muon-neutrino and Fe-muon anti-neutrino data in a model independent way" A. Bodek et al. [CCFR/NuTe Collaboration]. hep-ex/0005021

712. "Measurement of $J / \psi$ and $\psi(2 S)$ polarization in $p \bar{p}$ collisions at $\sqrt{s}=1.8 \mathrm{TeV}$ " T. Affolder et al. [CDF Collaboration].

hep-ex/0004027

Phys. Rev. Lett. 85, 2886 (2000)

713. "Direct measurement of the $W$ boson width in $p \bar{p}$ collisions at $\sqrt{s}=1.8 \mathrm{TeV}$ " T. Affolder et al. [CDF Collaboration].

hep-ex/0004017

Phys. Rev. Lett. 85, 3347 (2000)

714. "Search for second and third generation leptoquarks including production via technicolor interactions in $p \bar{p}$ collisions at $\sqrt{s}=1.8 \mathrm{TeV}^{\prime \prime}$

T. Affolder et al. [CDF Collaboration].

hep-ex/0004003

Phys. Rev. Lett. 85, 2056 (2000)

715. "Studies of the response of the prototype CMS hadron calorimeter, including magnetic field effects, to pion, electron, and muon beams"

V. V. Abramov et al. [CMS-HCAL Collaboration].

hep-ex/0007045

Nucl. Instrum. Meth. A 457, 75 (2001)

716. "Limits on gravitino production and new processes with large missing transverse energy in $p \bar{p}$ collisions at $\sqrt{s}=1.8 \mathrm{TeV}^{\prime \prime}$

T. Affolder et al. [CDF Collaboration].

hep-ex/0003026

Phys. Rev. Lett. 85, 1378 (2000)

717. "Diffractive dijets with a leading antiproton in $\bar{p} p$ collisions at $\sqrt{s}=1800 \mathbf{G e V}$ " T. Affolder et al. [CDF Collaboration].

Phys. Rev. Lett. 84, 5043 (2000).

718. "Search for new particles decaying to $t \bar{t}$ in $p \bar{p}$ collisions at $\sqrt{s}=1.8 \mathbf{T e V}$ "

T. Affolder et al. [CDF Collaboration].

hep-ex/0003005

Phys. Rev. Lett. 85, 2062 (2000)

719. "Searches for neutrino mass in the NuTeV experiment"

M. H. Shaevitz et al. [NuTeV Collaboration].

In *Cape Town 1999, Weak interactions and neutrinos* 400-404 
720. "Expression of interest for $\mathbf{R} \& \mathbf{D}$ towards a neutrino factory based on a storage ring and a muon collider"

D. Ayres et al. [Neutrino Factory and Muon Collider Collaboration].

physics/9911009 [physics.acc-ph]

721. "Reply to comment on parton distributions, $\mathbf{d} / \mathbf{u}$, and higher twist effects at high $\mathrm{x}$ "

U. -K. Yang and A. Bodek.

hep-ph/9912543

Phys. Rev. Lett. 84, 5456 (2000)

722. "Search for a 33.9-MeV/c**2 neutral particle in pion decay"

J. A. Formaggio et al. [NuTeV Collaboration].

hep-ex/9912062

Phys. Rev. Lett. 84, 4043 (2000)

723. "A measurement of the differential dijet mass cross section in $p \bar{p}$ collisions at $\sqrt{s}=1.8 \mathrm{TeV} "$

T. Affolder et al. [CDF Collaboration].

hep-ex/9912022

Phys. Rev. D 61, 091101 (2000)

724. "Search for scalar top quark production in $p \bar{p}$ collisions at $\sqrt{s}=1.8 \mathrm{TeV}$ "

T. Affolder et al. [CDF Collaboration].

hep-ex/9912018

Phys. Rev. Lett. 84, 5273 (2000)

725. "Observation of orbitally excited $B$ mesons in $p \bar{p}$ collisions at $\sqrt{s}=1.8 \mathrm{TeV}$ "

T. Affolder et al. [CDF Collaboration].

Phys. Rev. D 64, 072002 (2001).

726. "Search for the charged Higgs boson in the decays of top quark pairs in the $e \tau$ and $\mu \tau$ channels at $\sqrt{s}=1.8 \mathbf{T e V}^{\prime \prime}$

T. Affolder et al. [CDF Collaboration].

hep-ex/9912013

Phys. Rev. D 62, 012004 (2000)

727. "Search for scalar top and scalar bottom quarks in $p \bar{p}$ collisions at $\sqrt{s}=1.8$ TeV" T. Affolder et al. [CDF Collaboration].

hep-ex/9910049

Phys. Rev. Lett. 84, 5704 (2000)

728. "Production of $\Upsilon(1 S)$ mesons from $\chi_{b}$ decays in $p \bar{p}$ collisions at $\sqrt{s}=1.8$ TeV"

T. Affolder et al. [CDF Collaboration].

hep-ex/9910025

Phys. Rev. Lett. 84, 2094 (2000) 
729. "Measurement of $b$ quark fragmentation fractions in the production of strange and light $B$ mesons in $p \bar{p}$ collisions at $\sqrt{s}=1.8 \mathrm{TeV} "$

F. Abe et al. [CDF Collaboration].

Phys. Rev. D 60, 092005 (1999).

730. "Search for a $W^{\prime}$ boson via the decay mode $W^{\prime} \rightarrow \mu \nu_{\mu}$ in $1.8 \mathrm{TeV} p \bar{p}$ collisions" F. Abe et al. [CDF Collaboration].

hep-ex/9910004

Phys. Rev. Lett. 84, 5716 (2000)

731. "Measurement of the helicity of $W$ bosons in top quark decays"

T. Affolder et al. [CDF Collaboration].

hep-ex/9909042

Phys. Rev. Lett. 84, 216 (2000)

732. "Evidence for diffractive charm production in muon-neutrino Fe and anti-muonneutrino Fe scattering at the Tevatron"

T. Adams et al. [NuTeV Collaboration].

hep-ex/9909041

Phys. Rev. D 61, 092001 (2000)

733. "Search for a fourth-generation quark more massive than the $\mathbf{Z 0}$ boson in $p \bar{p}$ collisions at $\sqrt{s}=1.8 \mathrm{TeV}$ "

T. Affolder et al. [CDF Collaboration].

hep-ex/9909027

Phys. Rev. Lett. 84, 835 (2000)

734. "A preshower detector for the CDF plug upgrade: Test beam results"

M. G. Albrow, S. Aota, G. Apollinari, T. Asakawa, M. W. Bailey, P. de Barbaro, V. Barnes and D. Benjamin et al..

Nucl. Instrum. Meth. A 431, 104 (1999).

735. "Measurement of $b$ quark fragmentation fractions in $p \bar{p}$ collisions at $\sqrt{s}=1.8 \mathrm{TeV}$ " T. Affolder et al. [CDF Collaboration].

hep-ex/9909011

Phys. Rev. Lett. 84, 1663 (2000)

736. "A measurement of $\sin (2 \beta)$ from $B \rightarrow J / \psi K_{S}^{0}$ with the CDF detector" T. Affolder et al. [CDF Collaboration].

hep-ex/9909003

Phys. Rev. D 61, 072005 (2000)

737. "Low $\mathrm{Q}^{* *} \mathbf{2}$ low $\mathrm{x}$ structure function analysis of CCFR data for $\mathbf{F}(2)$ " B. T. Tamminga et al. [CCFR Collaboration].

hep-ex/9908064

Nucl. Phys. A 663, 344 (2000) 
738. "Studies of higher twist and higher order effects in NLO and NNLO QCD analysis of lepton nucleon scattering data on $F(2)$ and $R=\operatorname{sigma}(L) / \operatorname{sigma}(T) "$

U. -K. Yang and A. Bodek.

hep-ex/9908058

Eur. Phys. J. C 13, 241 (2000)

739. "Precision calibration of the NuTeV calorimeter"

D. A. Harris et al. [NuTeV Collaboration].

hep-ex/9908056

Nucl. Instrum. Meth. A 447, 377 (2000)

740. "The transverse momentum and total cross section of $e^{+} e^{-}$pairs in the $Z$ boson region from $p \bar{p}$ collisions at $\sqrt{s}=1.8 \mathbf{T e V}^{\prime \prime}$

T. Affolder et al. [CDF Collaboration].

hep-ex/0001021

Phys. Rev. Lett. 84, 845 (2000)

741. "Observation of diffractive beauty production at the Fermilab Tevatron"

T. Affolder et al. [CDF Collaboration].

Phys. Rev. Lett. 84, 232 (2000).

742. "Search for neutral heavy leptons in a high-energy neutrino beam"

A. Vaitaitis et al. [NuTeV and E815 Collaborations].

hep-ex/9908011

Phys. Rev. Lett. 83, 4943 (1999)

743. "Measurement of the $B^{0} \bar{B}^{0}$ oscillation frequency using $\ell^{-} D^{*+}$ pairs and lepton flavor tags"

T. Affolder et al. [CDF Collaboration].

hep-ex/9907053

Phys. Rev. D 60, 112004 (1999)

744. "A Measurement of ( $\mathrm{xF}$ neutrino(3)) (xF anti-neutrino(3)) and $\mathrm{R}$ with the CCFR detector"

U. -K. Yang et al. [CCFR-NuTeV Collaboration].

hep-ex/9906042

Nucl. Phys. Proc. Suppl. 79, 89 (1999)

745. "Strange content of the nucleon (NuTeV)"

T. Adams et al. [NuTeV Collaboration].

hep-ex/9906038

In *Bloomington 1999, Physics with a high luminosity polarized electron ion collider*337-346

746. "Charm production at NuTeV"

T. Adams et al. [NuTeV Collaboration].

hep-ex/9906037 
747. "A Measurement of $\sin ^{* * 2}$ theta(W) in neutrino $\mathbf{N}$ scattering from NuTeV" G. P. Zeller et al. [NuTeV Collaboration].

hep-ex/9906024

748. "Constraints on PDFs from $\mathrm{W}$ and $\mathrm{Z}$ rapidity distributions at CDF"

A. Bodek [CDF Collaboration].

Nucl. Phys. Proc. Suppl. 79, 136 (1999).

749. "Nuclear structure functions in the large $\mathrm{x}$ large $\mathrm{Q}^{* *} 2$ kinematic region in neutrino deep inelastic scattering"

M. Vakili et al. [CCFR Collaboration].

hep-ex/9905052

Phys. Rev. D 61, 052003 (2000)

750. "Search for color singlet technicolor particles in $p \bar{p}$ collisions at $\sqrt{s}=1.8 \mathrm{TeV}$ "

T. Affolder et al. [CDF Collaboration].

Phys. Rev. Lett. 84, 1110 (2000).

751. "Search for the flavor-changing neutral current decays $B^{+} \rightarrow \mu^{+} \mu^{-} K^{+}$and $B^{0} \rightarrow$ $\mu^{+} \mu^{-} K^{* 0}$

T. Affolder et al. [CDF Collaboration].

hep-ex/9905004

Phys. Rev. Lett. 83, 3378 (1999)

752. "Measurement of $\sin ^{* *} 2$ theta(W) in neutrino $\mathbf{N}$ scattering from $\mathbf{N u T e V}$ "

R. A. Johnson et al. [NuTeV Collaboration].

hep-ex/9904028

In *Cape Town 1999, Weak interactions and neutrinos* 62-66

753. "Implication of $\mathbf{W}$ boson charge asymmetry measurements in $\mathbf{p}$ anti-p collisions to models for charge symmetry violations in parton distributions"

A. Bodek, Q. Fan, M. Lancaster, K. S. McFarland and U. -K. Yang.

hep-ex/9904022

Phys. Rev. Lett. 83, 2892 (1999)

754. "Measurement of the $B_{d}^{0} \bar{B}_{d}^{0}$ oscillation frequency using dimuon data in $p \bar{p}$ collisions at $\sqrt{s}=1.8 \mathrm{TeV}$ "

F. Abe et al. [CDF Collaboration].

Phys. Rev. D 60, 051101 (1999).

755. "Measurement of $B^{0} \bar{B}^{0}$ flavor oscillations using jet-charge and lepton flavor tagging in $p \bar{p}$ collisions at $\sqrt{s}=1.8 \mathrm{TeV}^{\prime \prime}$

F. Abe et al. [CDF Collaboration].

hep-ex/9903011

Phys. Rev. D 60, 072003 (1999)

756. "Measurement of the associated $\gamma+\mu^{ \pm}$production cross section in $p \bar{p}$ collisions at $\sqrt{s}=1.8 \mathrm{TeV}^{\prime \prime}$ 
F. Abe et al. [CDF Collaboration].

hep-ex/9902001

Phys. Rev. D 60, 092003 (1999)

757. "Physics with high $\mathbf{P}(\mathrm{T})$ leptons at CDF: W asymmetry, high mass Drell-Yan and limits on quark lepton compositeness"

A. Bodek [CDF Collaboration].

Nucl. Phys. Proc. Suppl. 66, 96 (1998).

758. "Performance of the NuTeV Fe - scintillator sampling calorimeter and implications for thin calorimeters"

S. Avvakumov et al. [CCFR/NuTeV Collaboration].

hep-ex/0001047

Nucl. Phys. Proc. Suppl. 78, 232 (1999)

759. "A Study of an acrylic Cherenkov radiation detector"

B. Porter, P. S. Auchincloss, P. de Barbaro, A. Bodek and H. S. Budd.

physics/9908049

Am. J. Phys. 67, 1022 (1999)

760. "Searches for evidence of neutrino mass in the NuTeV experiment at Fermilab" T. Adams et al. [NuTeV Collaboration].

In *Vancouver 1998, High energy physics, vol. 1* 616-620

761. "Measurement of $\sin ^{2} \theta_{W}$ in $\nu N$ scattering at the Tevatron"

T. Adams et al. [ $\mathrm{NuTeV}$ Collaboration].

In *Vancouver 1998, High energy physics, vol. $1 *$ 555-559

762. "Heavy quark production in neutrino deep inelastic scattering"

T. Adams, A. Alton, C. G. Arroyo, S. Avvakumov, L. de Barbaro, P. de Barbaro, A. O. Bazarko and R. H. Bernstein et al..

In *Batavia 1998, Heavy quarks at fixed target* 198-206

763. "High $Q^{2}$ deep inelastic scattering at HERA"

A. M. Cooper-Sarkar, A. Bodek, K. Long, E. Rizvi and H. Spiesberger.

hep-ph/9902277

J. Phys. G G 25, 1387 (1999)

764. "A search for $B_{s}^{0} \bar{B}_{s}^{0}$ oscillations using the semileptonic decay $B_{s}^{0} \rightarrow \phi \ell^{+} X \nu$ " F. Abe et al. [CDF Collaboration].

Phys. Rev. Lett. 82, 3576 (1999).

765. "Measurement of $b \bar{b}$ rapidity correlations in $p \bar{p}$ collisions at $\sqrt{s}=1.8 \mathrm{TeV}$ " F. Abe et al. [CDF Collaboration].

Phys. Rev. D 61, 032001 (2000).

766. "Search for $R$-parity violating supersymmetry using like-sign dielectrons in $p \bar{p}$ collisions at $\sqrt{s}=1.8 \mathrm{TeV}$ " 
F. Abe et al. [CDF Collaboration].

hep-ex/9908063

Phys. Rev. Lett. 83, 2133 (1999)

767. "Search for neutral heavy leptons in the NuTeV experiment at Fermilab"

R. B. Drucker et al. [NuTeV Collaboration].

hep-ex/9811036

In *Vancouver 1998, High energy physics, vol. 2* 1370-1373

768. "Determination of alpha-s and measurements of $\mathrm{R}(\mathrm{L}), \mathrm{k}$, and $-\mathrm{V}(\mathrm{cs})$ - from neutrino nucleon DIS at CCFR"

J. Yu et al. [CCFR/NuTeV Collaboration].

In *Vancouver 1998, High energy physics, vol. 1* 835-839

769. "Events with a rapidity gap between jets in $\bar{p} p$ collisions at $\sqrt{s}=630 \mathrm{GeV}$ "

F. Abe et al. [CDF Collaboration].

Phys. Rev. Lett. 81, 5278 (1998).

770. "Neutrino trident production from NuTeV"

T. Adams et al. [NuTeV Collaboration].

hep-ex/9811012

In *Vancouver 1998, High energy physics, vol. 1* 631-634

771. "Search for third-generation leptoquarks from technicolor models in $p \bar{p}$ collisions at $\sqrt{s}=1.8 \mathrm{TeV} "$

F. Abe et al. [CDF Collaboration].

Phys. Rev. Lett. 82, 3206 (1999).

772. "Performance of a prototype CMS hadron barrel calorimeter in a test beam"

A. Bodek.

hep-ex/9810033

IEEE Trans. Nucl. Sci. 46, 407 (1999)

773. "Kinematics of $t \bar{t}$ events at CDF"

F. Abe et al. [CDF Collaboration].

Phys. Rev. D 59, 092001 (1999).

774. "Search for a technicolor omega(T) particle in events with a photon and a b quark jet at CDF"

F. Abe et al. [CDF Collaboration].

hep-ex/9810031

Phys. Rev. Lett. 83, 3124 (1999)

775. "Measurement of the top quark mass with the collider detector at Fermilab"

F. Abe et al. [CDF Collaboration].

hep-ex/9810029

Phys. Rev. Lett. 82, 271 (1999), [Erratum-ibid. 82, 2808 (1999)] 
776. "Measurement of $Z^{0}$ and Drell-Yan production cross section using dimuons in $\bar{p} p$ collisions at $\sqrt{s}=1.8 \mathrm{TeV}$ "

F. Abe et al. [CDF Collaboration].

Phys. Rev. D 59, 052002 (1999).

777. "Parton distributions, $d / u$, and higher twist effects at high $\mathbf{x}$ "

U. -K. Yang and A. Bodek.

hep-ph/9809480

Phys. Rev. Lett. 82, 2467 (1999)

778. "A High statistics search for neutrino(e) (anti-neutrino(e)) - $i$ neutrino(tau) (anti-neutrino(tau)) oscillations"

D. Naples et al. [CCFR/NuTeV Collaboration].

hep-ex/9809023

Phys. Rev. D 59, 031101 (1999)

779. "Search for new particles decaying to $b \bar{b}$ in $p \bar{p}$ collisions at $\sqrt{s}=1.8 \mathrm{TeV}$ "

F. Abe et al. [CDF Collaboration].

hep-ex/9809022

Phys. Rev. Lett. 82, 2038 (1999)

780. "Search for the decays $B_{s}^{0}, B_{d}^{0} \rightarrow e^{ \pm} \mu^{ \pm}$and Pati-Salam leptoquarks"

F. Abe et al. [CDF Collaboration].

Phys. Rev. Lett. 81, 5742 (1998).

781. "Measurement of the lepton charge asymmetry in $W$ boson decays produced in $p \bar{p}$ collisions"

F. Abe et al. [CDF Collaboration].

hep-ex/9809001

Phys. Rev. Lett. 81, 5754 (1998)

782. "Search for Higgs bosons produced in association with a vector boson in $p \bar{p}$ collisions at $\sqrt{s}=1.8 \mathrm{TeV}^{\prime \prime}$

F. Abe et al. [CDF Collaboration].

Phys. Rev. Lett. 81, 5748 (1998).

783. "A Measurement of alpha(s) $\left(\mathrm{Q}^{* *} 2\right)$ from the Gross-Llewellyn Smith sum rule" J. H. Kim, D. A. Harris, C. G. Arroyo, L. de Barbaro, P. de Barbaro, A. O. Bazarko, R. H. Bernstein and A. Bodek et al.

hep-ex/9808015

Phys. Rev. Lett. 81, 3595 (1998)

784. "Measurement of the $B_{s}^{0}$ meson lifetime using semileptonic decays"

F. Abe et al. [CDF Collaboration].

hep-ex/9808003

Phys. Rev. D 59, 032004 (1999) 
785. "Search for second generation leptoquarks in the dimuon plus dijet channel of $p \bar{p}$ collisions at $\sqrt{s}=1.8 \mathrm{TeV}$ "

F. Abe et al. [CDF Collaboration].

hep-ex/9810024

Phys. Rev. Lett. 81, 4806 (1998)

786. "Searches for new physics in diphoton events in $p \bar{p}$ collisions at $\sqrt{s}=1.8 \mathrm{TeV}$ "

F. Abe et al. [CDF Collaboration].

hep-ex/9806034

Phys. Rev. D 59, 092002 (1999)

787. "Measurement of the $B_{d}^{0}-\bar{B}_{d}^{0}$ flavor oscillation frequency and study of same side flavor tagging of $B$ mesons in $p \bar{p}$ collisions"

F. Abe et al. [CDF Collaboration].

hep-ex/9806026

Phys. Rev. D 59, 032001 (1999)

788. "Parton distributions at high $X$ "

U. -K. Yang and A. Bodek.

hep-ph $/ 9806458$

In *Brussels 1998, Deep inelastic scattering and QCD* 234-241

789. "Measurement of the CP-violation parameter $\sin (2 \beta)$ in $B_{d}^{0} / \bar{B}_{d}^{0} \rightarrow J / \psi K_{S}^{0}$ decays" F. Abe et al. [CDF Collaboration].

hep-ex/9806025

Phys. Rev. Lett. 81, 5513 (1998)

790. "Measurements of the longitudinal structure function and $-\mathrm{V}(\mathrm{cs})$ - in the CCFR experiment"

U. -K. Yang et al. [CCFR/NuTeV Collaboration].

hep-ex/9806023

In *Brussels 1998, Deep inelastic scattering and QCD* 131-136

791. "Parton distributions, $d-u$ and higher twists at high $X$ "

U. -K. Yang, A. Bodek and Q. Fan.

hep-ph/9806457

792. "Improved measurement of the $B^{-}$and $\bar{B}^{0}$ meson lifetimes using semileptonic decays"

F. Abe et al. [CDF Collaboration].

hep-ex/9806018

Phys. Rev. D 58, 092002 (1998)

793. "Measurement of $\sin ^{* *} 2$ theta-W from neutrino nucleon scattering at $\mathrm{NuTeV}$ " K. S. McFarland et al. [NuTeV Collaboration].

hep-ex/9806013 
794. "Measurement of the $W$ boson production asymmetry and $W$ boson width at the Tevatron"

A. Bodek [CDF Collaboration].

795. "Observation of the $B_{c}$ meson in $p \bar{p}$ collisions at $\sqrt{s}=1.8 \mathrm{TeV}$ "

F. Abe et al. [CDF Collaboration].

hep-ex/9805034

Phys. Rev. Lett. 81, 2432 (1998)

796. "Search for long-lived parents of $Z^{0}$ bosons in $p \bar{p}$ collisions at $\sqrt{s}=1.8$ TeV"

F. Abe et al. [CDF Collaboration].

hep-ex/9805017

Phys. Rev. D 58, 051102 (1998)

797. "Measurement of the $\sigma(W+\geq 1 \mathrm{jet}) / \sigma(W)$ cross section ratio from $\bar{p} p$ collisions at $\sqrt{s}=1.8 \mathrm{TeV}^{\prime}$

F. Abe et al. [CDF Collaboration].

Phys. Rev. Lett. 81, 1367 (1998).

798. "Observation of $B_{c}$ mesons in $p \bar{p}$ collisions at $\sqrt{s}=1.8 \mathbf{T e V}$ "

F. Abe et al. [CDF Collaboration].

hep-ex/9804014

Phys. Rev. D 58, 112004 (1998)

799. "Search for the rare decay $W^{ \pm} \rightarrow D_{s}^{+-} \gamma$ in $p \bar{p}$ collisions at $\sqrt{s}=1.8 \mathbf{T e V}$ "

F. Abe et al. [CDF Collaboration].

Phys. Rev. D 58, 091101 (1998).

800. "Search for the rare decay $W^{ \pm} \rightarrow \pi^{ \pm}+\gamma$ in $p \bar{p}$ collisions at $\sqrt{s}=1.8 \mathbf{T e V}$ "

F. Abe et al. [CDF Collaboration].

Phys. Rev. D 58, 031101 (1998).

801. "Observation of $B^{+} \rightarrow \psi(2 S) K^{+}$and $B^{0} \rightarrow \psi(2 S) K^{* 0}(892)$ decays and measurements of $B$ meson branching fractions into $J / \psi$ and $\psi(2 S)$ final states"

F. Abe et al. [CDF Collaboration].

hep-ex/9803013

Phys. Rev. D 58, 072001 (1998)

802. "Search for chargino - neutralino associated production at the Fermilab Tevatron collider"

F. Abe et al. [CDF Collaboration].

hep-ex/9803015

Phys. Rev. Lett. 80, 5275 (1998)

803. "Searches for new physics in diphoton events in $p \bar{p}$ collisions at $\sqrt{s}=1.8 \mathrm{TeV}$ "

F. Abe et al. [CDF Collaboration].

hep-ex/9801019

Phys. Rev. Lett. 81, 1791 (1998) 
804. "Search for the decays $B_{d}^{0} \rightarrow \mu^{+} \mu^{-}$and $B_{s}^{0} \rightarrow \mu^{+} \mu^{-}$in $p \bar{p}$ collisions at $\sqrt{s}=1.8$ TeV"

F. Abe et al. [CDF Collaboration].

Phys. Rev. D 57, 3811 (1998).

805. "Physics with high $\mathbf{P}(\mathrm{T})$ leptons at CDF: W asymmetry, high mass Drell-Yan and limits on quark lepton compositeness"

A. Bodek.

806. "Fiber R and D for the CMS HCAL"

H. S. Budd, A. Bodek, P. de Barbaro, D. Ruggiero and E. Skup.

AIP Conf. Proc. 450, 83 (1998).

807. "Precision measurement of $\mathbf{R}=\operatorname{Sigma}(\mathbf{L}) / \operatorname{Sigma}(\mathbf{T})$ and $\mathbf{F} 2$ in deep inelastic electron scattering"

A. Bodek, S. Dasu, P. de Barbaro, H. Harada, M. W. Krasny, K. Lang, R. C. Walker and B. Filippone et al..

In *Arles 1988, Multiparticle dynamics* 225-229

808. "Jet pseudorapidity distribution in direct photon events in $p \bar{p}$ collisions at $\sqrt{s}=$ 1.8 TeV"

F. Abe et al. [CDF Collaboration].

Phys. Rev. D 57, 1359 (1998).

809. "Observation of hadronic $\mathbf{W}$ decays in $t \bar{t}$ events with the Collider Detector at Fermilab"

F. Abe et al. [CDF Collaboration].

hep-ex/9711004

Phys. Rev. Lett. 80, 5720 (1998)

810. "Measurement of $B$ hadron lifetimes using $J / \psi$ final states at CDF"

F. Abe et al. [CDF Collaboration].

Phys. Rev. D 57, 5382 (1998).

811. "Measurement of the top quark mass and $t \bar{t}$ production cross section from dilepton events at the Collider Detector at Fermilab"

F. Abe et al. [CDF Collaboration].

hep-ex/9802017

Phys. Rev. Lett. 80, 2779 (1998)

812. "Measurement of the top quark mass"

F. Abe et al. [CDF Collaboration].

hep-ex/9801014

Phys. Rev. Lett. 80, 2767 (1998)

813. "Measurement of the $t \bar{t}$ production cross section in $p \bar{p}$ collisions at $\sqrt{s}=1.8 \mathrm{TeV}$ " F. Abe et al. [CDF Collaboration].

hep-ex/9710008

Phys. Rev. Lett. 80, 2773 (1998) 
814. "Improved determination of alpha(s) from neutrino nucleon scattering" W. G. Seligman, C. G. Arroyo, L. de Barbaro, P. de Barbaro, A. O. Bazarko, R. H. Bernstein, A. Bodek and T. Bolton et al..

hep-ex/9701017

Phys. Rev. Lett. 79, 1213 (1997)

815. "Measurement of the $B^{0} \bar{B}^{0}$ oscillation frequency using $\pi B$ meson charge-flavor correlations in $p \bar{p}$ collisions at $\sqrt{s}=1.8 \mathbf{T e V}^{\prime \prime}$

F. Abe et al. [CDF Collaboration].

hep-ex/9712004

Phys. Rev. Lett. 80, 2057 (1998)

816. "Search for flavor-changing neutral current decays of the top quark in $p \bar{p}$ collisions at $\sqrt{s}=1.8 \mathrm{TeV} "$

F. Abe et al. [CDF Collaboration].

Phys. Rev. Lett. 80, 2525 (1998).

817. "Properties of jets in $W$ boson events from $1.8 \mathrm{TeV} \bar{p} p$ collisions"

F. Abe et al. [CDF Collaboration].

hep-ex/9709016

Phys. Rev. Lett. 79, 4760 (1997)

818. "Measurement of the differential cross section for events with large total transverse energy in $p \bar{p}$ collisions at $\sqrt{s}=1.8 \mathbf{T e V}^{\prime}$

F. Abe et al. [CDF Collaboration].

Phys. Rev. Lett. 80, 3461 (1998).

819. "Dijet production by color - singlet exchange at the Fermilab Tevatron"

F. Abe et al. [CDF Collaboration].

Phys. Rev. Lett. 80, 1156 (1998).

820. "Search for first generation leptoquark pair production in $p \bar{p}$ collisions at $\sqrt{s}=1.8$ TeV"

F. Abe et al. [CDF Collaboration].

hep-ex/9708017

Phys. Rev. Lett. 79, 4327 (1997)

821. "Search for new particles decaying into $b \bar{b}$ and produced in association with $W$ bosons decaying into $e \nu$ or $\mu \nu$ at the Tevatron"

F. Abe et al. [CDF Collaboration].

Phys. Rev. Lett. 79, 3819 (1997).

822. "Limits on quark-lepton compositeness scales from dileptons produced in 1.8 TeV $p \bar{p}$ collisions"

F. Abe et al. [CDF Collaboration].

Phys. Rev. Lett. 79, 2198 (1997). 
823. "Search for new gauge bosons decaying into dileptons in $\bar{p} p$ collisions at $\sqrt{s}=1.8$ TeV"

F. Abe et al. [CDF Collaboration].

Phys. Rev. Lett. 79, 2192 (1997).

824. "Properties of photon plus two-jet events in $\bar{p} p$ collisions at $\sqrt{s}=1.8$ TeV"

F. Abe et al. [CDF Collaboration].

Phys. Rev. D 57, 67 (1998).

825. "Properties of six-jet events with large six-jet mass at the Fermilab $p \bar{p}$ collider" F. Abe et al. [CDF Collaboration].

Phys. Rev. D 56, 2532 (1997).

826. "Double parton scattering in $\bar{p} p$ collisions at $\sqrt{s}=1.8 \mathrm{TeV}$ "

F. Abe et al. [CDF Collaboration].

Phys. Rev. D 56, 3811 (1997).

827. "The mu tau and e tau decays of top quark pairs produced in $p \bar{p}$ collisions at $\sqrt{s}=1.8 \mathbf{T e V}^{\prime}$

F. Abe et al. [CDF Collaboration].

hep-ex/9704007

Phys. Rev. Lett. 79, 3585 (1997)

828. "Measurement of double parton scattering in $\bar{p} p$ collisions at $\sqrt{s}=1.8 \mathrm{TeV}$ "

F. Abe et al. [CDF Collaboration].

Phys. Rev. Lett. 79, 584 (1997).

829. "Search for charged Higgs decays of the top quark using hadronic decays of the tau lepton"

F. Abe et al. [CDF Collaboration].

hep-ex/9704003

Phys. Rev. Lett. 79, 357 (1997)

830. "First observation of the all hadronic decay of $t \bar{t}$ pairs"

F. Abe et al. [CDF Collaboration].

Phys. Rev. Lett. 79, 1992 (1997).

831. "Measurement of diffractive dijet production at the Tevatron"

F. Abe et al. [CDF Collaboration].

Phys. Rev. Lett. 79, 2636 (1997).

832. "Fiber R and D for the CMS HCAL"

H. S. Budd, A. Bodek, P. de Barbaro, D. Ruggiero and E. Skup.

hep-ex/0102031

833. "Production of $J / \psi$ mesons from $\chi_{c}$ meson decays in $p \bar{p}$ collisions at $\sqrt{s}=1.8$ TeV"

F. Abe et al. [CDF Collaboration].

Phys. Rev. Lett. 79, 578 (1997). 
834. "Search for gluinos and squarks at the Fermilab Tevatron Collider" F. Abe et al. [CDF Collaboration].

Phys. Rev. D 56, 1357 (1997).

835. " $J / \psi$ and $\psi(2 S)$ production in $p \bar{p}$ collisions at $\sqrt{s}=1.8 \mathbf{T e V}$ "

F. Abe et al. [CDF Collaboration].

Phys. Rev. Lett. 79, 572 (1997).

836. "Observation of diffractive $W$ boson production at the Tevatron"

F. Abe et al. [CDF Collaboration].

hep-ex/9703010

Phys. Rev. Lett. 78, 2698 (1997)

837. "Search for new particles decaying to dijets at CDF"

F. Abe et al. [CDF Collaboration].

hep-ex/9702004

Phys. Rev. D 55, 5263 (1997)

838. "A Precision measurement of electroweak parameters in neutrino - nucleon scattering"

K. S. McFarland et al. [CCFR and E744 and E770 Collaborations].

hep-ex/9701010

Eur. Phys. J. C 1, 509 (1998)

839. "Measuring the energy of cosmic ray muons in a sampling calorimeter"

L. G. Bugel, A. P. Chikkatur, A. Bodek, P. S. Auchincloss, P. de Barbaro, H. S. Budd, D. A. Harris and W. K. Sakumoto et al..

840. "A measurement of $\mathbf{R}=\operatorname{sigma}(\mathbf{L}) / \operatorname{sigma}(\mathbf{T})$ in deep inelastic neutrino nucleon scattering at the Tevatron"

A. Bodek et al. [CCFR/NuTeV Collaboration].

841. "Limits on $\mathbf{n u} / \mathbf{m u}-\mathbf{i} \mathbf{n u} / \mathbf{t a u}, \mathbf{n u} / \mathbf{e}$ oscillations from precision measurement of nu N neutral current interactions"

D. Naples, A. Romosan, C. G. Arroyo, P. S. Auchincloss, P. de Barbaro, A. O. Bazarko, R. H. Bernstein and A. Bodek et al..

842. "Drell-Yan production and limits on quark substructure using dilepton events in anti-p p collisions at CDF"

B. J. Kim, P. de Barbaro, A. Bodek, H. S. Budd, Q. Fan, M. Pillai and W. K. Sakumoto.

843. "Neutrino production of same sign dimuons at the TEVATRON"

W. H. Smith, K. T. Bachmann, R. H. Bernstein, R. E. Blair, C. Foudas, W. C. Lefmann, W. C. Leung and S. R. Mishra et al..

Conf. Proc. C 8708101, 537 (1987).

844. "The Collider Detector at Fermilab: Collected physics papers"

F. Abe et al. [CDF Collaboration]. 
845. "Study of magnetic field influence on hadron calorimeter response"

V. Abramov, N. Akchurin, K. Arisaka, P. Baillon, A. H. Ball, P. de Barbaro, V. Barnes and G. Bayatian et al..

846. "The CDF-II detector: Technical design report"

R. Blair et al. [CDF Collaboration].

847. "Search for third generation leptoquarks in $\bar{p} p$ collisions at $\sqrt{s}=1.8$ TeV" F. Abe et al. [CDF Collaboration].

Phys. Rev. Lett. 78, 2906 (1997).

848. "A High statistics search for muon-neutrino (anti-muon-neutrino) - $i$ electronneutrino (anti-electron-neutrino) oscillations in the small mixing angle regime" A. Romosan et al. [CCFR/NuTeV Collaboration]. hep-ex/9611013

Phys. Rev. Lett. 78, 2912 (1997)

849. "Physics with Ws, Zs and leptons at the Tevatron Collider"

A. Bodek [CDF Collaboration].

Acta Phys. Polon. B 28, 477 (1997).

850. "Sensitivity to quark and lepton compositeness at the Tevatron"

P. de Barbaro et al. [CDF Collaboration].

eConf C 960625, NEW160 (1996).

851. "A New technique for determining charge and momentum of electrons and positrons using calorimetry and silicon tracking"

Q. Fan and A. Bodek.

Frascati Phys. Ser. 6, 553 (1996).

852. "Limits on quark - lepton compositeness and studies on $W$ asymmetry at the Tevatron Collider"

A. Bodek [CDF Collaboration].

853. "Measurement of dijet angular distributions at CDF"

F. Abe et al. [CDF Collaboration].

hep-ex/9609011

Phys. Rev. Lett. 77, 5336 (1996), [Erratum-ibid. 78, 4307 (1997)]

854. "Observation of $W^{+} W^{-}$production in $\bar{p} p$ collisions at $\sqrt{s}=1.8$ TeV"

F. Abe et al. [CDF Collaboration].

Phys. Rev. Lett. 78, 4536 (1997).

855. "Measurement of the branching fraction $B\left(B_{u}^{+} \rightarrow J / \psi \pi^{+}\right)$and search for $B_{c}^{+} \rightarrow$ $J / \psi \pi^{+} "$

F. Abe et al. [CDF Collaboration].

Phys. Rev. Lett. 77, 5176 (1996). 
856. "Observation of $\Lambda_{b}^{0} \rightarrow J / \psi \Lambda$ at the Fermilab proton antiproton collider" F. Abe et al. [CDF Collaboration].

Phys. Rev. D 55, 1142 (1997).

857. "Measurement of $b \bar{b}$ production correlations, $B^{0} \bar{B}^{0}$ mixing, and a limit on epsilon(B) in $p \bar{p}$ collisions at $\sqrt{s}=1.8 \mathbf{T e V}^{\prime \prime}$

F. Abe et al. [CDF Collaboration].

Phys. Rev. D 55, 2546 (1997).

858. "Updated electroweak measurements from neutrino - nucleon deeply inelastic scattering at CCFR"

K. S. McFarland et al. [CCFR/NuTeV Collaboration].

hep-ex/9608007

859. "A Search for Neutral Heavy Vector Gauge Bosons in $\bar{p} p$ Collisions at $s^{1 / 2}=$ 1.8-TeV"

M. K. Pillai et al. [CDF Collaboration].

hep-ex/9608006

In *Minneapolis 1996, Particles and fields, vol. 1* 282-284

860. "Quarks and gluons at hadron colliders"

A. Bodek [CDF Collaboration].

In *Rome 1996, Deep inelastic scattering and related phenomena* 59-76

861. "A Measurement of $\mathbf{R}=$ sigma-L / sigma- $\mathbf{T}$ in deep inelastic neutrino - nucleon scattering at the Tevatron"

A. Bodek et al. [CCFR/NuTeV Collaboration].

J. Phys. G G 22, 775 (1996).

862. "Measurement of the $\gamma+D^{* \pm}$ cross section in $\bar{p} p$ collisions at $\sqrt{s}=1.8 \mathrm{TeV}$ "

F. Abe et al. [CDF Collaboration].

Phys. Rev. Lett. 77, 5005 (1996).

863. "Ratios of bottom meson branching fractions involving $J / \psi$ mesons and determination of $b$ quark fragmentation fractions"

F. Abe et al. [CDF Collaboration].

hep-ex/9607003

Phys. Rev. D 54, 6596 (1996)

864. "Further properties of high mass multi - jet events at the Fermilab proton - antiproton collider"

F. Abe et al. [CDF Collaboration].

hep-ex/9605004

Phys. Rev. D 54, 4221 (1996)

865. "Measurement of the lifetime of the $B_{s}^{0}$ meson using the exclusive decay mode $B_{s}^{0} \rightarrow J / \psi \phi "$

F. Abe et al. [CDF Collaboration].

Phys. Rev. Lett. 77, 1945 (1996). 
866. "Measurement of $\Lambda_{b}^{0}$ lifetime using $\Lambda_{b}^{0} \rightarrow \Lambda_{c}^{+} \ell^{-} \bar{\nu}$ "

F. Abe et al. [CDF Collaboration].

Phys. Rev. Lett. 77, 1439 (1996).

867. "Forward-backward charge asymmetry of electron pairs above the $Z^{0}$ pole" F. Abe et al. [CDF Collaboration].

Phys. Rev. Lett. 77, 2616 (1996).

868. "Properties of jets in $\mathrm{Z}$ boson events from $1.8 \mathrm{TeV} \bar{p} p$ collisions"

F. Abe et al. [CDF Collaboration].

hep-ex/9603003

Phys. Rev. Lett. 77, 448 (1996)

869. "Search for flavor changing neutral current $B$ meson decays in $p \bar{p}$ collisions at $\sqrt{s}=1.8$ TeV"

F. Abe et al. [CDF Collaboration].

Phys. Rev. Lett. 76, 4675 (1996).

870. "Measurement of the $B^{-}$and $\bar{B}^{0}$ meson lifetimes using semileptonic decays" F. Abe et al. [CDF Collaboration].

Phys. Rev. Lett. 76, 4462 (1996).

871. "Search for chargino - neutralino production in $p \bar{p}$ collisions at $\sqrt{s}=1.8 \mathrm{TeV}$ " F. Abe et al. [CDF Collaboration].

hep-ex/9603001

Phys. Rev. Lett. 76, 4307 (1996)

872. "Inclusive jet cross section in $\bar{p} p$ collisions at $\sqrt{s}=1.8 \mathrm{TeV}$ "

F. Abe et al. [CDF Collaboration].

hep-ex/9601008

Phys. Rev. Lett. 77, 438 (1996)

873. "Limits on $\mathbf{n u} / \mathbf{m u}($ anti-nu/mu) - i nu/tau (anti-nu/tau) and nu/mu (anti-nu/mu) $-i$ nu/e (anti-nu/e) oscillations from a precision measurement of neutrino nucleon neutral current interactions"

K. S. McFarland, D. Naples, C. G. Arroyo, P. S. Auchincloss, P. de Barbaro, A. O. Bazarko, R. H. Bernstein and A. Bodek et al..

In *Brussels 1995, High energy physics* 530-531

874. "A measurement of alpha(s) $\left(Q^{* * 2}\right)$ from the Gross-Llewellyn Smith sum rule" J. H. Kim, D. A. Harris, C. G. Arroyo, P. S. Auchincloss, P. de Barbaro, A. O. Bazarko, R. H. Bernstein and A. Bodek et al..

In *Brussels 1995, High energy physics* 134-135

875. "A measurement of $\mathbf{R}(\mathbf{n u})$ at CCFR"

D. A. Harris, C. G. Arroyo, P. S. Auchincloss, P. de Barbaro, A. O. Bazarko, R. H. Bernstein, A. Bodek and T. Bolton et al..

In *Brussels 1995, High energy physics* 35-36 
876. "Study of two particle azimuthal correlations in e+ e- annihilation at $\mathrm{s}^{* *}(1 / 2)$ $=58-\mathrm{GeV}^{\prime \prime}$

S. Kanda et al. [AMY Collaboration].

Phys. Rev. D 52, 4872 (1995).

877. "A Determination of alpha-s in e+ e- annihilation at $\mathrm{s}^{* *}(\mathbf{1} / 2)=57.3-\mathrm{GeV}$ " Y. K. Li et al. [AMY Collaboration].

Phys. Lett. B 355, 394 (1995).

878. "A Measurement of $\mathbf{R}=$ sigma-L / sigma- $\mathbf{T}$ in deep inelastic neutrino - nucleon scattering at the Tevatron"

U. -K. Yang, P. S. Auchincloss, P. de Barbaro, A. Bodek, H. S. Budd, D. A. Harris, W. K. Sakumoto and W. C. Lefmann et al..

hep-ex/9605005

J. Phys. G G 22, 775 (1996)

879. "Search for the rare decay $W^{ \pm} \rightarrow \pi^{ \pm}+\gamma$ "

F. Abe et al. [CDF Collaboration].

Phys. Rev. Lett. 76, 2852 (1996).

880. "Search for charged Higgs decays of the top quark using hadronic tau decays" F. Abe et al. [CDF Collaboration].

hep-ex/9601003

Phys. Rev. D 54, 735 (1996)

881. "Search for gluino and squark cascade decays at the Fermilab Tevatron Collider" F. Abe et al. [CDF Collaboration].

Phys. Rev. Lett. 76, 2006 (1996).

882. "Reconstruction of $B^{0} \rightarrow J / \psi K_{s}^{0}$ and measurement of ratios of branching ratios involving $B \rightarrow J / \psi K^{*}$,

F. Abe et al. [CDF Collaboration].

Phys. Rev. Lett. 76, 2015 (1996).

883. "Constraints on parton distributions from CDF"

A. Bodek [CDF Collaboration].

884. "Measurement of charm production in two photon processes using inclusive lepton events at TRISTAN"

T. Aso et al. [AMY Collaboration].

Phys. Lett. B 363, 249 (1995).

885. "Measurement of the mass of the $B_{s}^{0}$ meson"

F. Abe et al. [CDF Collaboration].

Phys. Rev. D 53, 3496 (1996).

886. "Measurement of $\sigma \cdot B(W \rightarrow e \nu)$ and $\sigma \cdot B\left(Z^{0} \rightarrow e^{+} e^{-}\right)$in $p \bar{p}$ collisions at $\sqrt{s}=1.8$ TeV"

F. Abe et al. [CDF Collaboration]. 
hep-ex/9509010

Phys. Rev. Lett. 76, 3070 (1996)

887. "The CDF upgrade calorimeter"

J. Freeman et al. [CDF Collaboration].

In * Capri 1991, Calorimetry in high energy physics* 189-194

888. "Techniques for optical isolation and construction of multitile assemblies in scintillator tile - fiber calorimeters using white epoxy"

M. Olsson, P. de Barbaro, A. Bodek, H. S. Budd, P. Koehn, M. Pillai, W. Sakumoto and R. C. Walker et al..

Rochester U. - UR-1370 (94/09,rec.Jan.95) 15 p. Rochester U. - UR-1389 (94/10,rec.Jan.95) 206-230

889. "Measurement of correlated $\mu-\bar{b}$ jet cross-sections in $p \bar{p}$ collisions at $\sqrt{s}=1.8$ TeV"

F. Abe et al. [CDF Collaboration].

hep-ex/9508017

Phys. Rev. D 53, 1051 (1996)

890. " $\Upsilon$ production in $p \bar{p}$ collisions at $\sqrt{s}=1.8 \mathrm{TeV}$ "

F. Abe et al. [CDF Collaboration].

Phys. Rev. Lett. 75, 4358 (1995).

891. "Measurement of the polarization in the decays $B_{d} \rightarrow J / \psi K^{* 0}$ and $B_{s} \rightarrow J / \psi \phi$ "

F. Abe et al. [CDF Collaboration].

Phys. Rev. Lett. 75, 3068 (1995).

892. "Constraints on parton distributions in the nucleon from $W$ asymmetry and Drell-Yan collider data"

A. Bodek [CDF Collaboration].

In *Paris 1995, Deep inelastic scattering and QCD*239-244

893. "Determination of parton distributions in the nucleon in future experiments and facilities at Fermilab"

A. Bodek.

In *Paris 1995, Deep inelastic scattering and QCD* 531-534

894. "A Measurement of $\alpha^{-} s\left(Q^{2)}\right.$ from the Gross-Llewellyn-Smith sum rule"

D. A. Harris et al. [CCFR-NUTEV Collaboration].

hep-ex/9506010

In *Les Arcs 1995, QCD and high energy hadronic interactions* 247-250

895. "A Limit on $\nu_{\mu}$ (anti-muon-neutrino) $\rightarrow \nu_{\tau}$ (anti-tau-neutrino) oscillations from a precision measurement of neutrino - nucleon neutral current interactions"

K. S. McFarland, D. Naples, C. G. Arroyo, P. S. Auchincloss, P. de Barbaro, A. O. Bazarko, R. H. Bernstein and A. Bodek et al.

hep-ex/9506007

Phys. Rev. Lett. 75, 3993 (1995) 
896. "Study of $t \bar{t}$ production $p \bar{p}$ collisions using total transverse energy" F. Abe et al. [CDF Collaboration].

hep-ex/9506006

Phys. Rev. Lett. 75, 3997 (1995)

897. "A Measurement of Bose-Einstein correlations in e+ e- annihilation at TRISTAN"

S. K. Choi et al. [AMY Collaboration].

Phys. Lett. B 355, 406 (1995).

898. "A Measurement of the ratio $\sigma \times B(p \bar{p} \rightarrow W \rightarrow e \nu) / \sigma \times B\left(p \bar{p} \rightarrow Z^{0} \rightarrow e e\right)$ in $p \bar{p}$ collisions at $\sqrt{s}=1800 \mathrm{GeV}^{\prime \prime}$

F. Abe et al. [CDF Collaboration].

Phys. Rev. D 52, 2624 (1995).

899. "Identification of top quarks at CDF using kinematic variables"

F. Abe et al. [CDF Collaboration].

Phys. Rev. D 52, 2605 (1995).

900. "A Search for second generation leptoquarks in $p \bar{p}$ collisions at $\sqrt{s}=1.8$ TeV" F. Abe et al. [CDF Collaboration].

Phys. Rev. Lett. 75, 1012 (1995).

901. "Precision measurement of $\mathbf{R}=$ sigma-L / sigma- $\mathbf{T}$ on hydrogen, deuterium and beryllium targets in deep inelastic electron scattering"

L. H. Tao et al. [E140X Collaboration].

Z. Phys. C 70, 387 (1996).

902. "Measurement of the $B$ meson differential cross-section, $d \sigma / d p_{T}$, in $p \bar{p}$ collisions at $\sqrt{s}=1.8 \mathrm{TeV} "$

F. Abe et al. [CDF Collaboration].

hep-ex/9503013

Phys. Rev. Lett. 75, 1451 (1995)

903. "Neutrino results from the Fermilab tevatron"

M. H. Shaevitz et al. [CCFR Collaboration].

Nucl. Phys. Proc. Suppl. 38, 188 (1995).

904. "Properties of high mass multi - jet events at the Fermilab $p \bar{p}$ collider"

F. Abe et al. [CDF Collaboration].

Phys. Rev. Lett. 75, 608 (1995).

905. "Measurement of the $W$ boson mass"

F. Abe et al. [CDF Collaboration].

hep-ex/9503007

Phys. Rev. Lett. 75, 11 (1995) 
906. "Measurement of the $W$ boson mass"

F. Abe et al. [CDF Collaboration].

Phys. Rev. D 52, 4784 (1995).

907. "Limits on $W W Z$ and $W W \gamma$ couplings from $W W$ and $W Z$ production in $p \bar{p}$ collisions at $\sqrt{s}=1.8 \mathrm{TeV}$ "

F. Abe et al. [CDF Collaboration].

hep-ex/9503009

Phys. Rev. Lett. 75, 1017 (1995)

908. "Observation of top quark production in $\bar{p} p$ collisions"

F. Abe et al. [CDF Collaboration].

hep-ex/9503002

Phys. Rev. Lett. 74, 2626 (1995)

909. "A High $\mathrm{Q}^{* *} \mathbf{2}$ measurement of the photon structure function F2(gamma)"

S. K. Sahu et al. [AMY Collaboration].

Phys. Lett. B 346, 208 (1995).

910. "Measurement of the $B_{s}$ meson lifetime"

F. Abe et al. [CDF Collaboration].

hep-ex/9412017

Phys. Rev. Lett. 74, 4988 (1995)

911. "Search for new particles decaying to dijets in $p \bar{p}$ collisions at $\sqrt{s}=1.8 \mathrm{TeV}$ "

F. Abe et al. [CDF Collaboration].

hep-ex/9501001

Phys. Rev. Lett. 74, 3538 (1995)

912. "Search for the top quark decaying to a charged Higgs boson in $\bar{p} p$ collisions at $\sqrt{s}=1.8 \mathrm{TeV} "$

F. Abe et al. [CDF Collaboration].

Phys. Rev. Lett. 73, 2667 (1994).

913. "Simple formulae for light yield considerations in the design of scintillator Fe and scintillator $\mathrm{Pb}$ sampling calorimeters"

A. Bodek and P. S. Auchincloss.

Nucl. Instrum. Meth. A 357, 292 (1995).

914. "Measurement of the $B$ meson and $b$ quark cross-sections at $\sqrt{s}=1.8$ TeV using the exclusive decay $B^{0} \rightarrow J / \psi K^{* 0}(892) "$

F. Abe et al. [CDF Collaboration].

Phys. Rev. D 50, 4252 (1994).

915. "Search for charged bosons heavier than the $W$ in $p \bar{p}$ collisions at $\sqrt{s}=1800 \mathrm{GeV}$ " F. Abe et al. [CDF Collaboration].

Phys. Rev. Lett. 74, 2900 (1995). 
916. "Measurements of cross-section and asymmetry for $\mathrm{e}+\mathrm{e}--_{\mathbf{i}} \mathbf{b}$ anti-b and heavy quark fragmentation at KEK TRISTAN"

F. Liu et al. [AMY. Collaboration].

Phys. Rev. D 49, 4339 (1994).

917. "Kinematic evidence for top quark pair production in $W+$ multi - jet events in $p \bar{p}$ collisions at $\sqrt{s}=1.8 \mathrm{TeV}$ "

F. Abe et al. [CDF Collaboration].

hep-ex/9412009

Phys. Rev. D 51, 4623 (1995)

918. " $b \bar{b}$ quark pair correlations in $p \bar{p}$ collisions at $S^{(1 / 2)}=1.8-\mathbf{T e V}^{\prime}$

F. Abe et al. [CDF Collaboration].

Unpublished - incorporated into Phys.Rev. D55, 2546 (1997)

919. "CDF end plug upgrade: Scintillator tile fiber calorimeters for high-energy physics: Selected articles"

P. de Barbaro and A. Bodek.

920. "Limits on $Z$-photon couplings from $p \bar{p}$ interactions at $\sqrt{s}=1.8 \mathrm{TeV}$ "

F. Abe et al. [CDF Collaboration].

Phys. Rev. Lett. 74, 1941 (1995).

921. "The Charge asymmetry in $W$ boson decays produced in $p \bar{p}$ collisions at $\sqrt{s}=1.8$ TeV"

F. Abe et al. [CDF Collaboration].

hep-ex/9501008

Phys. Rev. Lett. 74, 850 (1995)

922. "Forward - backward charge asymmetry of quark pairs produced at the KEK TRISTAN e+ e- collider"

D. Stuart et al. [AMY. Collaboration].

Phys. Rev. D 49, 3098 (1994).

923. "Quality control studies of scintillating tile / fiber megatile production for the CDF end plug upgrade hadron calorimeter"

P. de Barbaro, K. Michaud, A. Bodek, H. S. Budd, Q. Fan, M. Olsson, M. Pillai and W. K. Sakumoto et al..

924. "A Direct measurement of the $W$ boson width"

F. Abe et al. [CDF Collaboration].

Phys. Rev. Lett. 74, 341 (1995).

925. "Recent results from the CCFR neutrino experiment at the Tevatron" W. H. Smith et al. [CCFR Collaboration].

926. "Measurement of $W-\gamma$ couplings with CDF in $p \bar{p}$ collisions at $\sqrt{s}=1.8 \mathrm{TeV}$ " F. Abe et al. [CDF Collaboration].

Phys. Rev. Lett. 74, 1936 (1995). 
927. "Measurement of $W+\gamma$ and $Z+\gamma$ cross-sections in the electron and muon channels in $S^{(1 / 2)}=1.8-\mathrm{TeV}$ anti-P P collisions"

F. Abe et al. [CDF Collaboration].

928. "A Comparison of theory and data from deep inelastic scattering experiments on $\mathbf{R}=$ sigma-L / sigma-T"

A. Bodek and U. -K. Yang.

In *Blois 1994, The heart of the matter* 531-534

929. "Tests of structure functions using leptons at CDF: $W$ asymmetry and Drell-Yan production"

A. Bodek et al. [CDF Collaboration].

930. "CDF plug upgrade hadron calorimeter design"

P. De Barbaro, A. Bodek, H. S. Budd, Q. Fan, P. Koehn, M. Olsson, M. Pillai and R. C. Walker et al..

931. "Search for radiative decays of neutralinos in $p \bar{p}$ collisions at $\sqrt{s}=1.8$ TeV" F. Abe et al. [CDF Collaboration].

Phys. Rev. Lett. 75, 613 (1995).

932. "Observation of rapidity gaps in $\bar{p} p$ collisions at $1.8 \mathrm{TeV}$ "

F. Abe et al. [CDF Collaboration].

Phys. Rev. Lett. 74, 855 (1995).

933. "A Precision measurement of the prompt photon cross-section in $p \bar{p}$ collisions at $\sqrt{s}=1.8 \mathrm{TeV} "$

F. Abe et al. [CDF Collaboration].

Phys. Rev. Lett. 73, 2662 (1994), [Erratum-ibid. 74, 1891 (1995)].

934. "Search for new gauge bosons decaying into dielectrons in $\bar{p} p$ collisions at $\sqrt{s}=1.8$ TeV"

F. Abe et al. [CDF Collaboration].

Phys. Rev. D 51, 949 (1995).

935. "Analysis of jet charged particle momentum distributions for quark - gluon separation in $\bar{p} p$ collisions at $\sqrt{s}=1.8-\mathrm{TeV}^{\prime}$

F. Abe et al. [CDF Collaboration].

936. "Tests of structure functions using leptons with CDF: $W$ asymmetry and DrellYan production"

A. Bodek [CDF Collaboration].

In *Eilat 1994, Proceedings, Deep inelastic scattering and related subjects* 278-293, and Rochester U. - UR-1351 (94/06,rec.Jul.) 16 p

937. "Determination of the strange quark content of the nucleon from a next-toleading order QCD analysis of neutrino charm production"

A. O. Bazarko et al. [CCFR Collaboration]. 
hep-ex/9406007

Z. Phys. C 65, 189 (1995)

938. "Evidence for color coherence in $p \bar{p}$ collisions at $\sqrt{s}=1.8 \mathrm{TeV}$ " F. Abe et al. [CDF Collaboration].

Phys. Rev. D 50, 5562 (1994).

939. " $W$ boson + jet angular distribution in $p \bar{p}$ collisions at $\sqrt{s}=1.8$ TeV" F. Abe et al. [CDF Collaboration].

Phys. Rev. Lett. 73, 2296 (1994).

940. "R \& D proposal: Proposal for research and development of a hadron calorimeter for high magnetic fields"

G. Bayatian et al. [RD43 Collaboration].

941. "Measurements of cross-section and charge asymmetry for e+ e- to mu+ muand $\mathrm{e}+\mathrm{e}-\mathrm{to}$ tau + tau- at $\mathrm{s}^{* *}(1 / 2)=57.8-\mathrm{GeV}^{\prime \prime}$

C. Velissaris et al. [AMY Collaboration].

Phys. Lett. B 331, 227 (1994).

942. "Measurement of the ratio $\sigma B(W \rightarrow e \nu) / \sigma B\left(Z^{0} \rightarrow e^{+} e^{-}\right)$in $\bar{p} p$ collisions at $\sqrt{s}=1.8$ TeV"

F. Abe et al. [CDF Collaboration].

Phys. Rev. Lett. 73, 220 (1994).

943. "Evidence for top quark production in $\bar{p} p$ collisions at $\sqrt{s}=1.8$ TeV"

F. Abe et al. [CDF Collaboration].

hep-ex/9405005

Phys. Rev. Lett. 73, 225 (1994)

944. "Evidence for top quark production in $\bar{p} p$ collisions at $\sqrt{s}=1.8 \mathbf{T e V}^{\prime}$

F. Abe et al. [CDF Collaboration].

Phys. Rev. D 50, 2966 (1994).

945. "Measurement of the $B^{+}$and $B^{0}$ meson lifetimes"

F. Abe et al. [CDF Collaboration].

Phys. Rev. Lett. 72, 3456 (1994).

946. "Measurements of the inclusive jet cross-section in photon photon interactions at TRISTAN"

B. J. Kim et al. [AMY Collaboration].

In *Tsukuba 1993, TRISTAN physics at high luminosities* 313-335

947. "Nuclear spin structure measurements at SLAC"

R. Arnold et al. [E143 Collaboration].

In *Calcutta 1993, Physics and astrophysics of quark-gluon plasma* 294-315

948. "Beam tests of composite calorimeter configurations from reconfigurable stack calorimeter" 
A. Beretvas et al. [SDC Collaboration].

Nucl. Instrum. Meth. A 329, 50 (1993).

949. "Measurement of the inclusive jet cross-section in photon-photon interactions at TRISTAN"

B. J. Kim et al. [AMY Collaboration].

Phys. Lett. B 325, 248 (1994).

950. "A Search for the top quark decaying to charged Higgs in $p \bar{p}$ collisions at $\sqrt{s}=1.8$ TeV"

F. Abe et al. [CDF Collaboration].

Phys. Rev. Lett. 72, 1977 (1994).

951. "Measurement of kinematic and nuclear dependence of $\mathbf{R}=$ sigma- $\mathrm{L} /$ sigma-t in deep inelastic electron scattering"

S. Dasu, P. deBarbaro, A. Bodek, H. Harada, M. W. Krasny, K. Lang, E. M. Riordan and L. Andivahis et al..

Phys. Rev. D 49, 5641 (1994).

952. "Measurements of the proton elastic form-factors for $1-\mathrm{GeV} / \mathrm{c}^{* *} \mathbf{2} \leq \mathrm{Q}^{* *} \mathbf{2} \leq 3$ $\mathrm{GeV} / \mathrm{C}^{* *} 2$ at SLAC"

R. C. Walker, B. Filippone, J. Jourdan, R. Milner, R. McKeown, D. H. Potterveld, L. Andivahis and R. Arnold et al..

Phys. Rev. D 49, 5671 (1994).

953. "A Precise measurement of the weak mixing angle in neutrino nucleon scattering" C. Arroyo et al. [CCFR Collaboration].

hep-ex/9405008

Phys. Rev. Lett. 72, 3452 (1994)

954. "Search for excited quarks in $p \bar{p}$ collisions at $\sqrt{s}=1.8 \mathbf{T e V}$ "

F. Abe et al. [CDF Collaboration].

Phys. Rev. Lett. 72, 3004 (1994).

955. "Studies of e+ e- annihilation multi - hadron events including muons at $\mathrm{s}^{* *}(1 / 2)$ $=57.8-\mathrm{GeV}^{\prime}$

F. Liu et al. [AMY Collaboration].

Submitted to: Phys. Rev. D..

956. "Tile/ Fiber results for the upgraded plug Hadron calorimeter"

P. Koehn, P. de Barbaro, A. Bodek, H. S. Budd, Q. Fan, M. Olsson, M. Pillai and W. K. Sakumoto et al..

957. "A Precision measurement of the weak mixing angle"

A. Bodek et al. [CCFR Collaboration].

In *Marseille 1993, Proceedings, High energy physics* 617-618. and Rochester Univ. - UR 1322 (93/10,rec.Nov.) 2 p. C 
958. "Measurement of the $\mathrm{x}, \mathrm{Q}^{* *} 2$ dependence of $\mathrm{R}=\operatorname{sigma}(\mathrm{L}) / \operatorname{sigma}(\mathrm{T})$ "

A. Bodek et al. [E140X Collaboration].

In *Marseille 1993, Proceedings, High energy physics* 127. and Rochester Univ. - UR 1321

(93/10,rec.Nov.) 1 p.

959. "A Study of the energy dependence of the mean, truncated mean, and most probable energy deposition of high-energy muons in sampling calorimeters"

P. S. Auchincloss, P. de Barbaro, A. Bodek, H. S. Budd, M. Pillai, F. Qun, W. K. Sakumoto and F. S. Merritt et al..

Nucl. Instrum. Meth. A 343, 463 (1994).

960. "Measurement of the $\bar{p} p$ total cross-section at $\sqrt{s}=546 \mathrm{GeV}$ and 1800-GeV"

F. Abe et al. [CDF Collaboration].

Phys. Rev. D 50, 5550 (1994).

961. "Measurement of small angle $\bar{p} p$ elastic scattering at $\sqrt{s}=546 \mathbf{G e V}$ and $\mathbf{1 8 0 0}$ GeV"

F. Abe et al. [CDF Collaboration].

Phys. Rev. D 50, 5518 (1994).

962. "Measurement of $\bar{p} p$ single diffraction dissociation at $\sqrt{s}=546 \mathbf{G e V}$ and $\mathbf{1 8 0 0}$ GeV"

F. Abe et al. [CDF Collaboration].

Phys. Rev. D 50, 5535 (1994).

963. "Top dilepton search at CDF"

F. Abe et al. [CDF Collaboration].

964. "Measurement of the A-dependence of deep inelastic electron scattering" J. Gomez, R. G. Arnold, P. E. Bosted, C. C. Chang, A. T. Katramatou, G. G. Petratos, A. A. Rahbar and S. E. Rock et al..

Phys. Rev. D 49, 4348 (1994).

965. "Probing the gluon distribution with the SS - OS dijet cross-section ratio"

F. Abe et al. [CDF Collaboration].

966. "Measurement of inclusive jet cross-sections in $p \bar{p}$ collisions at CDF"

F. Abe et al. [CDF Collaboration].

967. "SUSY search using trilepton events from $p \bar{p}$ collisions at $\sqrt{s}=\mathbf{1 . 8}-\mathbf{T e V}$ "

F. Abe et al. [CDF Collaboration].

968. "Measurement of the $B^{+}$and $B^{0}$ lifetimes"

F. Abe et al. [CDF Collaboration].

969. "The Lepton charge asymmetry in the decay of $W$ bosons produced in $p \bar{p}$ collisions at $\sqrt{s}=1.8-\mathrm{TeV}^{\prime \prime}$

F. Abe et al. [CDF Collaboration]. 
970. "Measurement of the $\mathbf{B}$ meson and $\mathbf{b}$ quark cross-sections in $p \bar{p}$ collisions at $\sqrt{s}=$ 1.8-TeV using the exclusive decays $B^{+} \rightarrow J / \psi K^{+}$and $B^{0} \rightarrow J / \psi K^{* 0}$ "

F. Abe et al. [CDF Collaboration].

971. "The Two jet differential cross-section at CDF"

F. Abe et al. [CDF Collaboration].

972. "Search for excited quarks in $p \bar{p}$ collisions at $\sqrt{s}=1.8-\mathrm{TeV}$ "

F. Abe et al. [CDF Collaboration].

973. "The Cross-section for the production of $b \bar{b}$ pairs in $p \bar{p}$ collisions at $\sqrt{s}=\mathbf{1 . 8 - T e V "}$ F. Abe et al. [CDF Collaboration].

974. "A Study of events with the highest total transverse energy in CDF" F. Abe et al. [CDF Collaboration].

975. "Direct photon results from CDF"

F. Abe et al. [CDF Collaboration].

976. "The Center-of-mass angular distribution of prompt photons produced in p antip collisions at $\sqrt{s}=1.8-\mathrm{TeV}^{\prime}$

F. Abe et al. [CDF Collaboration].

977. "A Search for new gauge bosons in $\bar{p} p$ collisions at $\sqrt{s}=1.8-\mathrm{TeV}$ in the dielectron decay mode"

F. Abe et al. [CDF Collaboration].

978. "Study of four jet events and evidence for double parton interactions in $p \bar{p}$ collisions at $\sqrt{s}=1.8 \mathrm{TeV}^{\prime \prime}$

F. Abe et al. [CDF Collaboration].

Phys. Rev. D 47, 4857 (1993).

979. "Measurements of inclusive jet production in almost real gamma gamma collisions at TRISTAN"

B. J. Kim et al. [AMY Collaboration].

980. "Inclusive $\chi_{c}$ and $b$ quark production in $\bar{p} p$ collisions at $\sqrt{s}=1.8 \mathrm{TeV}$ "

F. Abe et al. [CDF Collaboration].

Phys. Rev. Lett. 71, 2537 (1993).

981. "Search for quark compositeness, axigluons and heavy particles using the dijet invariant mass spectrum observed in $p \bar{p}$ collisions"

F. Abe et al. [CDF Collaboration].

Phys. Rev. Lett. 71, 2542 (1993).

982. "Measurement of alpha-s from the moment of particle momenta within jets from e+ e- annihilation"

K. B. Lee et al. [AMY Collaboration].

Phys. Lett. B 313, 469 (1993). 
983. "Measurement of the average lifetime of $\mathbf{B}$ hadrons produced in $p \bar{p}$ collisions at $\sqrt{s}=1.8 \mathrm{TeV}^{\prime \prime}$

F. Abe et al. [CDF Collaboration].

Phys. Rev. Lett. 71, 3421 (1993).

984. "Measurement of bottom quark production in 1.8 TeV $p \bar{p}$ collisions using semileptonic decay muons"

F. Abe et al. [CDF Collaboration].

Phys. Rev. Lett. 71, 2396 (1993).

985. "Measurement of Drell-Yan electron and muon pair differential cross-sections in $\bar{p} p$ collisions at $\sqrt{s}=1.8 \mathrm{TeV}$ "

F. Abe et al. [CDF Collaboration].

Phys. Rev. D 49, 1 (1994).

986. "Observation of the decay $B_{s}^{0} \rightarrow J / \psi \phi$ in $\bar{p} p$ collisions at $\sqrt{s}=1.8$ TeV"

F. Abe et al. [CDF Collaboration].

Phys. Rev. Lett. 71, 1685 (1993).

987. "A Search for first generation leptoquarks in $\bar{p} p$ collisions at $\sqrt{s}=1.8$ TeV"

F. Abe et al. [CDF Collaboration].

Phys. Rev. D 48, 3939 (1993).

988. "Measurement of the bottom quark production cross-section using semileptonic decay electrons in $p \bar{p}$ collisions at $\sqrt{s}=1.8 \mathbf{T e V}^{\prime \prime}$

F. Abe et al. [CDF Collaboration].

Phys. Rev. Lett. 71, 500 (1993).

989. "Measurement of jet multiplicity in $\mathbf{W}$ events produced in $p \bar{p}$ collisions at $\sqrt{s}=$ 1.8-Tev"

F. Abe et al. [CDF Collaboration].

Phys. Rev. Lett. 70, 4042 (1993).

990. “A Next-to-leading order QCD analysis of neutrino charm production: Probing nucleon strangeness"

A. O. Bazarko et al. [CCFR Collaboration].

In *Les Arcs 1993, Proceedings, QCD and high energy hadronic interactions* 43-46. and Columbia Univ. New York - Nevis R 1492 (93,rec.Jun.) 4 p. C

991. "A Precision measurement of $\sin ^{* *} 2$-theta $(w)$ in neutrino - nucleon scattering" B. J. King et al. [CCFR Collaboration].

In *Les Arcs 1993, Proceedings, '93 electroweak interactions and unified theories* 3-8. and Columbia Univ. New York - Nevis R 1489 (93,rec.Jun.) 6 p. C

992. "Measurement of the strange sea distribution using neutrino charm production" S. A. Rabinowitz, C. Arroyo, K. T. Bachmann, A. O. Bazarko, T. Bolton, C. Foudas, B. J. King and W. Lefmann et al..

Phys. Rev. Lett. 70, 134 (1993). 
993. "The Center-of-mass angular distribution of prompt photons produced in $p \bar{p}$ collisions at $\sqrt{s}=1.8 \mathrm{TeV}^{\prime \prime}$

F. Abe et al. [CDF Collaboration].

Phys. Rev. Lett. 71, 679 (1993).

994. "Measurement of the dijet mass distribution in $p \bar{p}$ collisions at $\sqrt{s}=1.8 \mathrm{TeV}$ " F. Abe et al. [CDF Collaboration].

Phys. Rev. D 48, 998 (1993).

995. "A Prompt photon cross-section measurement in $\bar{p} p$ collisions at $\sqrt{s}=1.8$ TeV" F. Abe et al. [CDF Collaboration].

Phys. Rev. D 48, 2998 (1993).

996. "Search for anomalous gamma gamma production at TRISTAN"

K. L. Sterner et al. [AMY Collaboration].

hep-ph/9302251

Phys. Lett. B 303, 385 (1993)

997. "Recent $\mathbf{R} \&$ \& results on tile / fiber calorimetry"

P. de Barbaro, A. Bodek, H. S. Budd, Q. Fan, P. Koehn, M. Olsson, M. Pillai and W. K. Sakumoto et al..

998. "Quantum chromodynamics fits to neutrino iron singlet and non-singlet structure functions"

W. G. Seligman, C. Arroyo, K. T. Bachmann, A. O. Bazarko, R. E. Blair, T. Bolton, C. Foudas and B. J. King et al..

999. "Measurement of the strange sea content of the nucleon by CCFR"

A. O. Bazarko, C. G. Arroyo, K. T. Bachmann, T. Bolton, C. Foudas, B. J. King, W. C. Lefmann and W. C. Leung et al..

1000. "Measurement of the weak mixing angle in neutrino nucleon scattering by CCFR" R. H. Bernstein, F. Borcherding, M. J. Lamm, W. L. Marsh, K. W. B. Merritt, H. Schellman, D. D. Yovanovitch and C. Arroyo et al..

1001. "Search for anomalous gamma gamma production at TRISTAN"

K. L. Sterner et al. [AMY Collaboration].

In ${ }^{*}$ Tsukuba 1992, TRISTAN physics at high luminosities* 82-99

1002. "Precision measurement of $\sin ^{* *} 2$ (Theta(W from nu Fe scattering at the Tevatron"

T. Bolton, C. Arroyo, K. T. Bachmann, A. O. Bazarko, R. E. Blair, C. Foudas, B. J. King and W. C. Lefmann et al..

Nucl. Phys. Proc. Suppl. 31, 278 (1992).

1003. "Structure function and QCD results from the CCFR neutrino experiment at the Fermilab Tevatron"

M. H. Shaevitz et al. [CCFR Collaboration]. 
1004. "Measurement of the cross-section for production of two isolated prompt photons in $\bar{p} p$ collisions at $\sqrt{s}=1.8 \mathbf{T e V}$ "

F. Abe et al. [CDF Collaboration].

Phys. Rev. Lett. 70, 2232 (1993).

1005. "Technical Design of a Detector (Solenoidal Detector) April 1, 1992 to be Operated at the Superconducting Super Collider"

G. H. Trilling et al. [Solenoidal Detector Collaboration].

1006. "Neutrino production of same sign dimuons at the Fermilab Tevatron"

W. K. Sakumoto et al. [CCFR Collaboration].

1007. "A Study of double vertex events in the neutrino - nucleon interactions"

P. de Barbaro et al. [CCFR Collaboration].

1008. "Comparison of jet production in $\bar{p} p$ collisions at $\sqrt{s}=546 \mathbf{G e V}$ and $1800 \mathrm{GeV}$ " F. Abe et al. [CDF Collaboration].

Phys. Rev. Lett. 70, 1376 (1993).

1009. "Search for $\Lambda_{b} \rightarrow J / \psi \Lambda^{0}$ in $p \bar{p}$ collisions at $\sqrt{s}=1.8$ TeV"

F. Abe et al. [CDF Collaboration].

Phys. Rev. D 47, 2639 (1993).

1010. "Neutrino scattering results from CCFR"

W. H. Smith, T. Kimmel, P. H. Sandler, C. Arroyo, K. T. Bachmann, A. O. Bazarko, R. E. Blair and T. Bolton et al..

Conf. Proc. C 9207131, 487 (1992).

1011. "A Study of double vertex events in the neutrino - nucleon interactions"

H. S. Budd et al. [CCFR Collaboration].

1012. "Inclusive $J / \psi, \psi(2 S)$ and $b$ quark production in $\bar{p} p$ collisions at $\sqrt{s}=1.8 \mathbf{T e V}^{\prime}$

F. Abe et al. [CDF Collaboration].

Phys. Rev. Lett. 69, 3704 (1992).

1013. "A Measurement of jet shapes in $p \bar{p}$ collisions at $\sqrt{s}=1.8 \mathbf{T e V}$ "

F. Abe et al. [CDF Collaboration].

Phys. Rev. Lett. 70, 713 (1993).

1014. "Search for squarks and gluinos from $\bar{p} p$ collisions at $\sqrt{s}=1.8$ TeV"

F. Abe et al. [CDF Collaboration].

Phys. Rev. Lett. 69, 3439 (1992).

1015. "Limit on the rare decay $W^{ \pm} \rightarrow \gamma \pi^{ \pm}$in $p \bar{p}$ collisions at $\sqrt{s}=1.8 \mathrm{TeV}$ "

F. Abe et al. [CDF Collaboration].

Phys. Rev. Lett. 69, 2160 (1992).

1016. "Neutrino production of dimuons at the Fermilab tevatron"

W. H. Smith, T. S. Kinnel, P. H. Sandler, C. Arroyo, K. T. Bachmann, A. O. Bazarko, 
R. E. Blair and C. Foudas et al..

Nucl. Phys. Proc. Suppl. 31, 262 (1993).

1017. "Search for right-handed coupling in neutrino N scattering"

S. R. Mishra, W. C. Leung, C. Arroyo, K. T. Bachmann, R. E. Blair, C. Foudas, B. J. King and W. C. Lefmann et al..

Phys. Rev. Lett. 68, 3499 (1992).

1018. "Measurements of nucleon structure functions, $F(2)\left(x, Q^{* *} 2\right)$ and $x F(3)(x$, $\mathrm{Q}^{* * 2}$ ), from the CCFR data: Tests of perturbative QCD"

A. Bodek, H. S. Budd, P. de Barbaro, W. K. Sakumoto, W. C. Leung, P. Z. Quintas, F. Sciulli and C. Arroyo et al..

In *La Thuile 1992, Proceedings, Results and perspectives in particle physics* $73-83$ and Rochester Univ. - UR 1261 (92/05,rec.Oct.) 11 p

1019. "Tests of perturbative QCD using CCFR data for measurements of nucleon structure functions"

A. Bodek, H. S. Budd, P. de Barbaro, W. K. Sakumoto, W. C. Leung, P. Z. Quintas, F. Sciulli and C. Arroyo et al..

Nucl. Phys. Proc. Suppl. 31, 271 (1993).

1020. "A Measurement of the Gross-Llewellyn-Smith sum rule from the CCFR x(F3) structure function"

W. C. Leung, P. Z. Quintas, S. R. Mishra, F. J. Sciulli, C. Arroyo, K. T. Bachmann, R. E. Blair and C. Foudas et al..

Phys. Lett. B 317, 655 (1993).

1021. "Measurements of nucleon structure functions, F2(x, $Q^{2)}$ and xF3(x, $Q^{2)}$, from the CCFR data: PQCD and the mean square charge test"

S. R. Mishra, W. C. Leung, P. Z. Quintas, F. J. Sciulli, C. Arroyo, K. T. Bachmann, R. E. Blair and C. Foudas et al..

1022. "A Measurement of Lambda (MS) from muon-neutrino - Fe nonsinglet structure functions at the Fermilab tevatron"

P. Z. Quintas, W. C. Leung, S. R. Mishra, F. J. Sciulli, C. Arroyo, K. T. Bachmann, R. E. Blair and C. Foudas et al..

Phys. Rev. Lett. 71, 1307 (1993).

1023. "The Dijet angular distribution in $p \bar{p}$ collisions at $\sqrt{s}=1.8 \mathbf{T e V}^{\prime}$

F. Abe et al. [CDF Collaboration].

Phys. Rev. Lett. 69, 2896 (1992).

1024. "Limits on the production of massive stable charged particles"

F. Abe et al. [CDF Collaboration].

Phys. Rev. D 46, 1889 (1992).

1025. "Nucleon structure functions from high energy neutrino interactions"

E. Oltman, P. S. Auchincloss, R. E. Blair, C. Haber, S. R. Mishra, M. Ruiz, F. J. Sciulli and 
M. H. Shaevitz et al..

Z. Phys. C 53, 51 (1992).

1026. "A Measurement of the production and muonic decay rate of $W$ and $Z$ bosons in $p \bar{p}$ collisions at $\sqrt{s}=1.8 \mathrm{TeV}$ "

F. Abe et al. [CDF Collaboration].

Phys. Rev. Lett. 69, 28 (1992).

1027. "A Scintillating fiber detector for electron and photon identification at high luminosity colliders"

J. Hauser, M. Lindgren, T. Muller, D. Neuberger, M. G. Albrow, G. Drake, J. Freeman and D. H. Kim et al.

Nucl. Instrum. Meth. A 321, 497 (1992).

1028. "Measurement of the isolated prompt photon cross-sections in $\bar{p} p$ collisions at $\sqrt{s}=1.8 \mathbf{T e V}^{\prime \prime}$

F. Abe et al. [CDF Collaboration].

Phys. Rev. Lett. 68, 2734 (1992).

1029. "A Measurement of the $B$ meson and $b$ quark cross-sections at $\sqrt{s}=1.8 \mathrm{TeV}$ using the exclusive decay $B^{ \pm} \rightarrow J / \psi K^{ \pm \prime}$

F. Abe et al. [CDF Collaboration].

Phys. Rev. Lett. 68, 3403 (1992).

1030. "Nucleon structure functions, F2 (x, $\left.\mathrm{Q}^{* *} 2\right)$ and $\mathrm{xF} 3\left(\mathrm{x}, \mathrm{Q}^{* *} 2\right)$, from Fe - neutrino scattering at the Fermilab tevatron"

S. R. Mishra et al. [CCFR Collaboration].

1031. "Precision measurements of nucleon structure functions, F2 and xF3, and tests of PQCD predictions"

S. R. Mishra et al. [CCFR Collaboration].

Conf. Proc. C 910725V1, 135 (1991).

1032. "Extraction of neutron to neutron structure function ratio"

A. Bodek, S. Dasu and S. E. Rock.

Conf. Proc. C 910725V1, 160 (1991).

1033. "Nucleon structure functions from muon-neutrino - Fe scattering at the Tevatron"

P. Z. Quintas et al. [CCFR Collaboration].

In *Tucson 1991, Proceedings, Intersections between particle and nuclear physics* $748-755$ and Rochester Univ. - UR 1211 (91,rec.Jan.92) 8 p

1034. "Measuring muon momenta with the CCFR neutrino detector"

B. J. King, C. G. Arroyo, K. T. Bachmann, R. E. Blair, C. Foudas, W. C. Lefmann, W. C. Leung and S. R. Mishra et al..

Nucl. Instrum. Meth. A 302, 254 (1991). 
1035. "Research and Development results on scintillating tile fiber calorimetry for the CDF and SDC detectors"

P. de Barbaro, A. Bodek, H. S. Budd, Q. Fan, W. K. Sakumoto, B. Winer, J. Proudfoot and D. G. Underwood et al..

Nucl. Instrum. Meth. A 315, 317 (1992).

1036. "Precision measurements of F2 (x, $\left.\mathrm{Q}^{* *} 2\right)$ and $\mathrm{xF} 3\left(\mathrm{x}, \mathrm{Q}^{* *} 2\right)$ by the CCFR Collaboration using muon-neutrino scattering at the Tevatron"

P. Z. Quintas et al. [CCFR Collaboration].

In *Les Arcs 1992, Perturbative QCD and hadronic interactions* 63-68

1037. "Recent electroweak results from the CCFR Collaboration: Neutrino tridents and W - Z interference and the Lorentz structure of the weak current"

S. R. Mishra et al. [CCFR Collaboration].

1038. "Precise measurements of the proton and deuteron structure functions from a global analysis of the SLAC deep inelastic electron scattering cross-sections"

L. W. Whitlow, E. M. Riordan, S. Dasu, S. Rock and A. Bodek.

Phys. Lett. B 282, 475 (1992).

1039. "Electroweak results from the CCFR experiment"

W. H. Smith et al. [CCFR Collaboration].

In *Geneva 1991, Joint international Lepton Photon Symposium, vol. 1*49-52

1040. "A Measurement of TeV muon energy loss in iron"

W. K. Sakumoto, P. de Barbaro, A. Bodek, H. S. Budd, B. J. Kim, F. S. Merritt, M. J. Oreglia and H. Schellman et al..

Phys. Rev. D 45, 3042 (1992).

1041. "Study of nuclear effects in the deuteron and extraction of neutron to proton structure function ratio"

A. Bodek, S. Dasu and S. Rock.

In *Tucson 1991, Proceedings, Intersections between particle and nuclear physics* 768-770

and SLAC Stanford - SLAC-PUB-5598 (91/06,rec.Aug.) 4 p

1042. "Evidence for hard scattering of hadronic constituents of photons in photonphoton collisions at TRISTAN"

R. Tanaka et al. [AMY Collaboration].

Phys. Lett. B 277, 215 (1992).

1043. "Neutrino tridents and W Z interference"

S. R. Mishra et al. [CCFR Collaboration].

Phys. Rev. Lett. 66, 3117 (1991).

1044. "Test beam results from prototype scintillating tile calorimeters with fiber readout"

W. K. Sakumuto, P. de Barbaro, V. E. Barnes, A. Bodek, H. S. Budd, G. W. Foster, J. Freeman and N. Giokaris et al..

Rochester Univ. - UR 1201 (91/01,rec.Feb.) 7 p. 
1045. "Proposal for beam test of scintillator calorimeter prototypes at Fermilab during FY 1991"

E. I. Rosenberg, V. Guarino, R. Hagstrom, N. Hill, J. Nasiatka, L. E. Price, J. Proudfoot and H. Spinka et al..

1046. "Test beam results from prototype scintillating tile calorimeters with fiber readout"

W. K. Sakumoto, P. de Barbaro, V. E. Barnes, A. Bodek, H. S. Budd, G. W. Foster, J. Freeman and N. Giokaris et al..

In *Batavia 1990, Proceedings, Calorimetry in high energy physics* 337-343

1047. "Comparison of Hadronic Shower Punchthrough and TeV dE/dx with Calculation"

B. J. Kim et al. [SDC Collaboration].

1048. "A Measurement of the neutral current electroweak parameters using the Fermilab narrow band neutrino beam"

P. G. Reutens, F. S. Merritt, M. J. Oreglia, P. S. Auchincloss, R. E. Blair, C. Haber, S. R. Mishra and E. Oltman et al..

Z. Phys. C 45, 539 (1990).

1049. "Letter of Intent by the Solenoidal Detector Collaboration to Construct and Operate A Detector at the Superconducting Super Collider"

G. H. Trilling et al. [Solenoid Detector Collaboration].

1050. "Comparison of hadronic shower punchthrough and TeV muon dE / dx with calculation"

B. J. Kim, P. de Barbaro, A. Bodek, H. S. Budd, W. K. Sakumoto, F. S. Merritt, M. J. Oreglia and H. Schellman et al..

Nucl. Phys. Proc. Suppl. 23B, 37 (1991).

1051. "Measurements of heavy quark fragmentation at TRISTAN"

S. S. Myung et al. [AMY Collaboration].

1052. "Nucleon structure functions from muon-neutrino - Fe scattering at the Tevatron"

W. H. Smith, P. H. Sandler, F. S. Merritt, M. J. Oreglia, B. A. Schumm, C. Arroyo, K. T. Bachmann and R. E. Blair et al..

Nucl. Phys. Proc. Suppl. 19, 281 (1991).

1053. "Comparison of the next-to-leading logarithm QCD approximation with TRISTAN data and a determination of $\Lambda_{M S}$ "

H. Sagawa et al. [AMY Collaboration].

1054. "A Search for charged Higgs bosons in $e^{+} e^{-}$annihilation using the AMY detector at $\sqrt{s}=50 \mathrm{GeV}-\mathbf{6 1 . 4} \mathrm{GeV} "$

J. Vinson et al. [AMY Collaboration]. 
1055. "Comparison of quark and gluon jets using three jet events from $e^{+} e^{-}$annihilation at TRISTAN"

Y. -K. Kim et al. [AMY Collaboration].

1056. "A Measurement of the photon structure function $F_{2}$ "

T. Sasaki et al. [AMY Collaboration].

Phys. Lett. B 252, 491 (1990).

1057. "Measurement of the polarization of $\tau$ leptons from $e^{+} e^{-}$at $\sqrt{s}=57 \mathrm{GeV}^{\text {" }}$

M. H. Lee et al. [AMY Collaboration].

1058. "Hadronic Shower Punchthrough And Tev Muon De/dx"

W. K. Sakumoto, P. de Barbaro, A. Bodek, H. S. Budd, B. J. Kim, F. S. Merritt, M. J. Oreglia and H. Schellman et al..

1059. "Bose-Einstein correlations in pion production at TRISTAN"

R. Walker et al. [AMY Collaboration].

1060. "Measurement of the $e^{+} e^{-} \rightarrow b \bar{b}$ cross-section and forward - backward charge asymmetry at a center-of-mass energy of $57.2 \mathrm{GeV}$ "

J. Lim et al. [AMY Collaboration].

1061. "Expression of Interest by the Solenoidal Detector Collaboration to Construct and Operate A Detector at the Superconducting Super Collider"

G. H. Trilling et al. [Solenoid Detector Collaboration].

1062. "A Precise extraction of $\mathbf{R}=$ sigma-L / sigma-T from a global analysis of the SLAC deep inelastic e p and e d scattering cross-sections"

L. W. Whitlow, S. Rock, A. Bodek, E. M. Riordan and S. Dasu.

Phys. Lett. B 250, 193 (1990).

1063. "Mass limits of charged Higgs at large $\tan (\beta)$ from $e^{+} e^{-}$annihilations at $\sqrt{s}=50$ GeV - 60.8 GeV"

J. R. Smith et al. [AMY Collaboration].

Phys. Rev. D 42, 949 (1990).

1064. "A Search for neutral heavy leptons in muon-neutrino - N interactions"

P. de Barbaro et al. [CCFR Collaboration].

1065. "A Precision Measurement Of The Gross-llewellyn-smith Sum Rule In Neutrino - $n$ Scattering At The Fermilab Tevatron"

W. -C. Leung, K. T. Bachmann, R. E. Blair, C. Foudas, B. J. King, W. C. Lefmann, S. R. Mishra and E. Oltman et al..

1066. "Inverse Muon Decay, Muon-neutrino E - i Mu- Electron-neutrino, At The Fermilab Tevatron"

S. R. Mishra, K. T. Bachmann, R. E. Blair, C. Foudas, B. J. King, W. C. Lefmann, W. C. Leung and E. Oltman et al..

Phys. Lett. B 252, 170 (1990). 
1067. "Precise Extractions Of The $\mathbf{X}$ And $\mathbf{Q}^{* *} \mathbf{2}$ Dependence $\mathrm{Of} \mathbf{R}=$ Sigma-l / Sigmatotal, F (2 P) F (2 D), And F (2 N / 2 P) From A Combined Analysis Of Slac Deep Inelastic Electron Scattering Experiments"

L. W. Whitlow, S. Rock, A. Bodek, E. M. Riordan and S. Dasu.

1068. "Charged hadron multiplicities in $e^{+} e^{-}$annihilations at $\sqrt{s}=50 \mathrm{GeV}-\mathbf{6 1 . 4} \mathbf{G e V}$ " H. W. Zheng et al. [AMY Collaboration].

Phys. Rev. D 42, 737 (1990).

1069. "Calibration of the CCFR Target Calorimeter"

W. K. Sakumoto, P. De Barbaro, A. Bodek, H. S. Budd, B. J. Kim, F. S. Merritt, M. J. Oreglia and H. Schellman et al..

Nucl. Instrum. Meth. A 294, 179 (1990).

1070. "Observation of anomalous production of muon pairs in $e^{+} e^{-}$annihilation into four lepton final states"

Y. H. Ho et al. [AMY Collaboration].

Phys. Lett. B 244, 573 (1990).

1071. "Measurements of $R$ for $e^{+} e^{-}$annihilation at TRISTAN"

T. Kumita et al. [AMY Collaboration].

Phys. Rev. D 42, 1339 (1990).

1072. "Measurement Of The Proton Elastic Form-factors For Q**2 = 1-gev/c**2 - 3gev/c**2"

R. C. Walker, B. W. Filippone, J. Jourdan, R. Milner, R. Mckeown, D. H. Potterveld, R. Arnold and D. Benton et al..

Phys. Lett. B 224, 353 (1989), [Erratum-ibid. B 240, 522 (1990)].

1073. "A search for leptoquark and colored lepton pair production in $e^{+} e^{-}$annihilations at TRISTAN"

G. N. Kim et al. [AMY Collaboration].

Phys. Lett. B 240, 243 (1990).

1074. "Multi - hadron event properties in $e^{+} e^{-}$annihilation at $\sqrt{s}=52 \mathrm{GeV}$ to 57-GeV" Y. K. Li et al. [AMY Collaboration].

Phys. Rev. D 41, 2675 (1990).

1075. "Forward - backward charge asymmetry in $e^{+} e^{-} \rightarrow$ hadron jets"

D. Stuart et al. [AMY Collaboration].

Phys. Rev. Lett. 64, 983 (1990).

1076. "A search for susy particles in $e^{+} e^{-}$annihilations at $\sqrt{s}=50 \mathrm{GeV}-\mathbf{6 0 . 8} \mathbf{G e V}$ " Y. Sakai et al. [AMY Collaboration].

Phys. Lett. B 234, 534 (1990).

1077. "Inverse Muon Decay And Neutrino Dimuon Production At The Tevatron"

S. R. Mishra et al. [CCFR Collaboration].

Nucl. Phys. Proc. Suppl. 13, 340 (1990). 
1078. "A COMBINED ANALYSIS OF SLAC EXPERIMENTS ON DEEP INELASTIC e p AND e d SCATTERING"

L. W. Whitlow, A. Bodek, S. Rock, J. Alster, R. G. Arnold, P. De Barbaro, D. Benton and P. E. Bosted et al..

Nucl. Phys. Proc. Suppl. 16, 215 (1990).

1079. "Tests of a calorimetric technique for measuring the energy of cosmic ray muons in the TeV energy range"

A. P. Chikkatur et al. [NuTeV/CCFR Collaboration].

Z. Phys. C 74, 279 (1997).

1080. "Testing QCD at CCFR: Three structure functions at once"

D. A. Harris, W. G. Seligman, C. G. Arroyo, P. S. Auchincloss, L. de Barbaro, P. de Barbaro, A. O. Bazarko and R. H. Bernstein et al.

1081. "CDF end plug hadron calorimeter upgrade: Design, production and quality control results"

P. de Barbaro, A. Bodek, H. S. Budd, Q. Fan, T. Haelen, B. J. Kim, P. Koehn and K. Michaud et al..

IEEE Trans. Nucl. Sci. 42, 510 (1995).

1082. "A Review of experimental data on the proton and neutron elastic form-factors" A. Bodek.

In *Blois 1994, The heart of the matter* 255-264

1083. "Study of light yield and uniformity in hadron calorimeters utilizing tile / fiber technology"

P. de Barbaro, A. Bodek, H. S. Budd, P. Koehn, M. Pillai, W. Sakumoto, J. Freeman and S. Gourlay.

1084. "The Effects of tile miscalibrations on the performance of the tile / fiber based hadron calorimeters"

P. de Barbaro, A. Bodek and B. Winer.

1085. "QCD analysis of neutrino scattering results from CCFR"

W. H. Smith, T. Kinnel, P. H. Sandler, C. G. Arroyo, B. J. King, K. T. Bachmann, A. O. Bazarko and T. Bolton et al..

In *Marseille 1993, High energy physics* 130-131

1086. "Hadron shower energy and direction measurements using drift chambers"

T. Kinnel, P. H. Sandler, W. H. Smith, C. Arroyo, K. T. Bachmann, A. O. Bazarko, T. Bolton and C. Foudas et al..

Nucl. Instrum. Meth. A 340, 474 (1994), [Erratum-ibid. A 345, 609 (1994)], [Erratum-ibid. A 365, 607 (1995)].

1087. "Neutrino production of same sign dimuons at the Fermilab Tevatron"

P. H. Sandler, T. S. Kinnel, W. H. Smith, K. Bachmann, R. Blair, C. Foudas, B. King and W. C. Lefmann et al..

Z. Phys. C 57, 1 (1993). 
1088. "Measurements of the nucleon spin structure at SLAC in end station A" R. G. Arnold et al. [E143 Collaboration].

1089. "Nucleon structure functions from muon-neutrino Fe scattering at the tevatron" P. Z. Quintas et al. [CCFR Collaboration].

In *Batavia 1990, Proceedings, Hadron structure functions and parton distributions* 50-57 and Columbia Univ. New York - Nevis R 1427 (90,rec.Jul.) 10 p

1090. "Measurement Of The Inclusive Charged Current Cross-section For Neutrino And Anti-neutrino Scattering On Isoscalar Nucleons"

P. S. Auchincloss, R. Blair, C. Haber, E. Oltman, W. C. Leung, M. Ruiz, S. R. Mishra and P. Z. Quintas et al..

Z. Phys. C 48, 411 (1990).

1091. "HADRON SHOWER PUNCHTHROUGH AND MUON PRODUCTION BY HADRONS OF 40-GeV, 70-GeV AND 100-GeV"

P. H. Sandler, W. H. Smith, K. Bachmann, R. Blair, C. Foudas, B. King, W. C. Lefmann and W. C. Leubng et al..

Phys. Rev. D 42, 759 (1990).

1092. "Neutrino Production of Opposite Sign Dimuons at Tevatron Energies"

C. Foudas, K. T. Bachmann, R. H. Bernstein, R. E. Blair, W. C. Lefmann, W. C. Leung, S. R. Mishra and E. Oltman et al..

Phys. Rev. Lett. 64, 1207 (1990).

endenumerate enddocument

1093. "Measurement of the ratio of three-jet to two-jet cross sections in $p \bar{p}$ collisions at $\sqrt{s}=1.96 \mathbf{T e V}^{\prime}$

V. M. Abazov et al. [D0 Collaboration].

arXiv:1209.1140 [hep-ex]

1094. "Measurement of the semileptonic charge asymmetry in B0 meson mixing with the Do detector"

V. M. Abazov et al. [D0 Collaboration].

arXiv:1208.5813 [hep-ex]

1095. "Limits on anomalous trilinear gauge boson couplings from $W W, W Z$ and $W \gamma$ production in $p \bar{p}$ collisions at $\sqrt{s}=1.96 \mathrm{TeV}^{\prime \prime}$

V. M. Abazov et al. [D0 Collaboration].

arXiv:1208.5458 [hep-ex]

1096. "Search for the standard model Higgs boson in associated $W H$ production in 9.7 $\mathrm{fb}^{-1}$ of $p \bar{p}$ collisions with the Do detector"

V. M. Abazov et al. [D0 Collaboration].

arXiv:1208.0653 [hep-ex] 
1097. "Combined search for the standard model Higgs boson decaying to $b \bar{b}$ using the Do Run II data set"

V. M. Abazov et al. [D0 Collaboration].

arXiv:1207.6631 [hep-ex]

1098. "Search for the standard model Higgs boson in $Z H \rightarrow \ell^{+} \ell^{-} b \bar{b}$ production with the D0 detector in $9.7 \mathrm{fb}^{-1}$ of $p \bar{p}$ collisions at $\sqrt{s}=1.96 \mathrm{TeV}$ "

V. M. Abazov et al. [D0 Collaboration].

arXiv:1207.5819 [hep-ex]

1099. "Search for the standard model Higgs boson in the $Z H \rightarrow \nu \bar{\nu} b \bar{b}$ channel in 9.5 $\mathrm{fb}^{-1}$ of $p \bar{p}$ collisions at $\sqrt{s}=1.96 \mathrm{TeV}^{\prime \prime}$

V. M. Abazov et al. [D0 Collaboration].

arXiv:1207.5689 [hep-ex]

1100. "Measurement of angular correlations of jets at $\operatorname{sqrt}(\mathrm{s})=1.96 \mathrm{TeV}$ and determination of the strong coupling at high momentum transfers"

V. M. Abazov et al. [D0 Collaboration].

arXiv:1207.4957 [hep-ex]

1101. "Measurement of the semileptonic charge asymmetry using $B_{s}^{0} \rightarrow D_{s} \mu X$ decays" V. M. Abazov et al. [D0 Collaboration].

arXiv:1207.1769 [hep-ex]

1102. "Search for Higgs boson production in oppositely charged dilepton and missing energy events in $p \bar{p}$ collisions at $\sqrt{s}=1.96 \mathrm{TeV}^{\prime \prime}$

V. M. Abazov et al. [D0 Collaboration].

arXiv:1207.1041 [hep-ex]

Phys. Rev. D 86, 032010 (2012)

1103. "Updated Combination of Searches for the Standard Model Higgs Boson at the Do Experiment in $9.7 \mathrm{fb}^{-1}$ of Data"

V. M. Abazov et al. [D0 Collaboration].

arXiv:1207.0422 [hep-ex]

1104. "Measurement of Leptonic Asymmetries and Top Quark Polarization in $t \bar{t}$ Production"

V. M. Abazov et al. [D0 Collaboration].

arXiv:1207.0364 [hep-ex]

1105. "Measurement of the differential cross section $d \sigma / d t$ in elastic $p \bar{p}$ scattering at $\sqrt{s}=1.96$ TeV"

V. M. Abazov et al. [D0 Collaboration].

arXiv:1206.0687 [hep-ex] 
1106. "Study of the decay $B_{s}^{0} \rightarrow J / \psi f_{2}^{\prime}(1525)$ in $\mu^{+} \mu^{-} K^{+} K^{-}$final states" V. M. Abazov et al. [D0 Collaboration].

arXiv:1204.5723 [hep-ex]

1107. "Measurement of the $\Lambda_{b}^{0}$ lifetime in the exclusive decay $\Lambda_{b}^{0} \rightarrow J / \psi \Lambda^{0}$ in $p \bar{p}$ collisions at $\sqrt{s}=1.96$ TeV"

V. M. Abazov et al. [D0 Collaboration].

arXiv:1204.2340 [hep-ex]

Phys. Rev. D 85, 112003 (2012)

1108. "Combination of searches for anomalous top quark couplings with $5.4 \mathrm{fb}^{-1}$ of $p \bar{p}$ collisions"

V. M. Abazov et al. [D0 Collaboration].

arXiv:1204.2332 [hep-ex]

Phys. Lett. B 713, 165 (2012)

1109. "Search for violation of Lorentz invariance in top quark pair production and decay"

V. M. Abazov et al. [D0 Collaboration].

arXiv:1203.6106 [hep-ex]

Phys. Rev. Lett. 108, 261603 (2012)

1110. "Observation of a narrow mass state decaying into $\Upsilon(1 S)+\gamma$ in $p \bar{p}$ collisions at $\sqrt{s}=1.96 \mathbf{T e V}^{\prime}$

V. M. Abazov et al. [D0 Collaboration].

arXiv:1203.6034 [hep-ex]

Phys. Rev. D 86, 031103 (2012)

1111. "Measurement of the photon $+b$-jet production differential cross section in $p \bar{p}$ collisions at $\sqrt{s}=1.96 \mathrm{TeV}^{\prime \prime}$

V. M. Abazov et al. [D0 Collaboration].

arXiv:1203.5865 [hep-ex]

Phys. Lett. B 714, 32 (2012)

1112. "Search for Zgamma events with large missing transverse energy in $p \bar{p}$ collisions at $\operatorname{sqrt}(\mathrm{s})=1.96 \mathrm{TeV}^{\prime \prime}$

V. M. Abazov et al. [D0 Collaboration].

arXiv:1203.5311 [hep-ex]

1113. "Search for the standard model Higgs boson in tau lepton pair final states" V. M. Abazov et al. [D0 Collaboration]. arXiv:1203.4443 [hep-ex]

Phys. Lett. B 714, 237 (2012)

1114. "Search for $W H$ associated production in $p \bar{p}$ collisions at $\sqrt{s}=1.96 \mathrm{TeV}$ " V. M. Abazov et al. [D0 Collaboration]. 
arXiv:1203.1082 [hep-ex]

Phys. Rev. D 86, 032005 (2012)

1115. "Measurement of the W Boson Mass with the Do Detector"

V. M. Abazov et al. [D0 Collaboration].

arXiv:1203.0293 [hep-ex]

Phys. Rev. Lett. 108, 151804 (2012)

1116. "Search for pair production of the scalar top quark in muon+tau final states"

V. M. Abazov et al. [D0 Collaboration].

arXiv:1202.1978 [hep-ex]

Phys. Lett. B 710, 578 (2012)

1117. "A measurement of the $W Z$ and $Z Z$ production cross sections using leptonic final states in $8.6 \mathrm{fb}^{-1}$ of $p \bar{p}$ collisions"

V. M. Abazov et al. [D0 Collaboration].

arXiv:1201.5652 [hep-ex]

Phys. Rev. D 85, 112005 (2012)

1118. "Measurement of the top quark mass in $p \bar{p}$ collisions using events with two leptons"

V. M. Abazov et al. [D0 Collaboration].

arXiv:1201.5172 [hep-ex]

1119. "An Improved determination of the width of the top quark"

V. M. Abazov et al. [D0 Collaboration].

arXiv:1201.4156 [hep-ex]

Phys. Rev. D 85, 091104 (2012)

1120. "Search for Higgs bosons of the minimal supersymmetric standard model in $p \bar{p}$ collisions at $\sqrt{(s)}=1.96$ TeV"

V. M. Abazov et al. [D0 Collaboration].

arXiv:1112.5431 [hep-ex]

Phys. Lett. B 710, 569 (2012)

1121. "Search for universal extra dimensions in $p \bar{p}$ collisions"

V. M. Abazov et al. [D0 Collaboration].

arXiv:1112.4092 [hep-ex]

Phys. Rev. Lett. 108, 131802 (2012)

1122. "Measurements of $W W$ and $W Z$ production in $W+$ jets final states in $p \bar{p}$ collisions"

V. M. Abazov et al. [D0 Collaboration].

arXiv:1112.0536 [hep-ex]

Phys. Rev. Lett. 108, 181803 (2012)

1123. " $Z \gamma$ production and limits on anomalous $Z Z \gamma$ and $Z \gamma \gamma$ couplings in $p \bar{p}$ collisions at $\sqrt{s}=1.96 \mathrm{TeV}^{\prime \prime}$ 
V. M. Abazov et al. [D0 Collaboration].

arXiv:1111.3684 [hep-ex]

Phys. Rev. D 85, 052001 (2012)

1124. "Search for a Narrow $t \bar{t}$ Resonance in $p \bar{p}$ Collisions at $\sqrt{s}=1.96$ TeV"

V. M. Abazov et al. [D0 Collaboration].

arXiv:1111.1271 [hep-ex]

Phys. Rev. D 85, 051101 (2012)

1125. "Search for anomalous $W t b$ couplings in single top quark production in $p \bar{p}$ collisions at $\sqrt{s}=1.96 \mathbf{T e V}^{\prime}$

V. M. Abazov et al. [D0 Collaboration].

arXiv:1110.4592 [hep-ex]

Phys. Lett. B 708, 21 (2012)

1126. "Measurement of the relative branching ratio of $B_{s}^{0} t o J / \psi f_{0}(980) \rightarrow B_{s}^{0} \rightarrow J / \psi \phi$ "

V. M. Abazov et al. [D0 Collaboration].

arXiv:1110.4272 [hep-ex]

Phys. Rev. D 85, 011103 (2012)

1127. "Evidence for spin correlation in $t \bar{t}$ production"

V. M. Abazov et al. [D0 Collaboration].

arXiv:1110.4194 [hep-ex]

Phys. Rev. Lett. 108, 032004 (2012)

1128. "Measurement of the inclusive jet cross section in $p \bar{p}$ collisions at $\sqrt{s}=1.96 \mathrm{TeV}$ " V. M. Abazov et al. [D0 Collaboration].

arXiv:1110.3771 [hep-ex]

Phys. Rev. D 85, 052006 (2012)

1129. "A Search for charged massive long-lived particles"

V. M. Abazov et al. [D0 Collaboration].

arXiv:1110.3302 [hep-ex]

Phys. Rev. Lett. 108, 121802 (2012)

1130. " $W \gamma$ production and limits on anomalous $W W \gamma$ couplings in $p \bar{p}$ collisions"

V. M. Abazov et al. [D0 Collaboration].

arXiv:1109.4432 [hep-ex]

Phys. Rev. Lett. 107, 241803 (2011)

1131. "Measurement of the CP-violating phase $\phi_{s}^{J / \psi \phi}$ using the flavor-tagged decay $B_{s}^{0} \rightarrow J / \psi \phi$ in $\mathbf{8} \mathbf{f b}^{-1}$ of $p \bar{p}$ collisions"

V. M. Abazov et al. [D0 Collaboration].

arXiv:1109.3166 [hep-ex]

Phys. Rev. D 85, 032006 (2012)

1132. "Model independent search for new phenomena in $p \bar{p}$ collisions at $\sqrt{s}=1.96 \mathrm{TeV}$ " V. M. Abazov et al. [D0 Collaboration]. 
arXiv:1108.5362 [hep-ex]

Phys. Rev. D 85, 092015 (2012)

1133. "Measurements of single top quark production cross sections and $\left|V_{t b}\right|$ in $p \bar{p}$ collisions at $\sqrt{s}=1.96 \mathrm{TeV}$ "

V. M. Abazov et al. [D0 Collaboration].

arXiv:1108.3091 [hep-ex]

Phys. Rev. D 84, 112001 (2011)

1134. "Forward-backward asymmetry in top quark-antiquark production"

V. M. Abazov et al. [D0 Collaboration].

arXiv:1107.4995 [hep-ex]

Phys. Rev. D 84, 112005 (2011)

1135. "Search for the standard model and a fermiophobic Higgs boson in diphoton final states"

V. M. Abazov et al. [D0 Collaboration].

arXiv:1107.4587 [hep-ex]

Phys. Rev. Lett. 107, 151801 (2011)

1136. "Search for first generation leptoquark pair production in the electron + missing energy + jets final state"

V. M. Abazov et al. [D0 Collaboration].

arXiv:1107.1849 [hep-ex]

Phys. Rev. D 84, 071104 (2011)

1137. "Search for associated Higgs boson production using like charge dilepton events in $p \bar{p}$ collisions at $\sqrt{s}=1.96 \mathrm{TeV}$ "

V. M. Abazov et al. [D0 Collaboration].

arXiv:1107.1268 [hep-ex]

Phys. Rev. D 84, 092002 (2011)

1138. "Measurement of the anomalous like-sign dimuon charge asymmetry with $9 \mathrm{fb}^{-1}$ of p pbar collisions"

V. M. Abazov et al. [D0 Collaboration].

arXiv:1106.6308 [hep-ex]

Phys. Rev. D 84, 052007 (2011)

1139. "Precision measurement of the ratio $\mathrm{B}(t \rightarrow W b) / \mathrm{B}(t \rightarrow W q)$ and Extraction of $V_{t b}$ " V. M. Abazov et al. [D0 Collaboration].

arXiv:1106.5436 [hep-ex]

Phys. Rev. Lett. 107, 121802 (2011)

1140. "Search for neutral Minimal Supersymmetric Standard Model Higgs bosons decaying to tau pairs produced in association with $b$ quarks in $p \bar{p}$ collisions at $\sqrt{s}=1.96 \mathbf{T e V}^{\prime \prime}$

V. M. Abazov et al. [D0 Collaboration]. 
arXiv:1106.4885 [hep-ex]

Phys. Rev. Lett. 107, 121801 (2011)

1141. "Search for Higgs bosons decaying to $\tau \tau$ pairs in $p \bar{p}$ collisions at $\sqrt{s}=1.96 \mathrm{TeV}$ " V. M. Abazov et al. [D0 Collaboration]. arXiv:1106.4555 [hep-ex]

Phys. Lett. B 707, 323 (2012)

1142. "Search for doubly-charged Higgs boson pair production in $p \bar{p}$ collisions at $\sqrt{s}=$ 1.96 TeV"

V. M. Abazov et al. [D0 Collaboration].

arXiv:1106.4250 [hep-ex]

Phys. Rev. Lett. 108, 021801 (2012)

1143. "Bounds on an anomalous dijet resonance in $W+$ jets production in $p \bar{p}$ collisions at $\sqrt{s}=1.96 \mathbf{T e V}^{\prime}$

V. M. Abazov et al. [D0 Collaboration].

arXiv:1106.1921 [hep-ex]

Phys. Rev. Lett. 107, 011804 (2011)

1144. "Direct measurement of the mass difference between top and antitop quarks"

V. M. Abazov et al. [D0 Collaboration].

arXiv:1106.2063 [hep-ex]

Phys. Rev. D 84, 052005 (2011)

1145. "Measurements of inclusive $W+$ jets production rates as a function of jet transverse momentum in $p \bar{p}$ collisions at $\sqrt{s}=1.96 \mathrm{TeV}^{\prime \prime}$

V. M. Abazov et al. [D0 Collaboration].

arXiv:1106.1457 [hep-ex]

Phys. Lett. B 705, 200 (2011)

1146. "Precise measurement of the top-quark mass from lepton+jets events at Do"

V. M. Abazov et al. [D0 Collaboration].

arXiv:1105.6287 [hep-ex]

Phys. Rev. D 84, 032004 (2011)

1147. "Measurement of the $t \bar{t}$ production cross section using dilepton events in $p \bar{p}$ collisions"

V. M. Abazov et al. [D0 Collaboration].

arXiv:1105.5384 [hep-ex]

Phys. Lett. B 704, 403 (2011)

1148. "Model-independent measurement of $t$-channel single top quark production in $p \bar{p}$ collisions at $\sqrt{s}=1.96 \mathrm{TeV}$ "

V. M. Abazov et al. [D0 Collaboration].

arXiv:1105.2788 [hep-ex]

Phys. Lett. B 705, 313 (2011) 
1149. "Measurement of the production fraction times branching fraction $f\left(b \rightarrow \Lambda_{b}\right)$. $\mathcal{B}(* L \rightarrow \mathcal{J} / \psi *) "$

V. M. Abazov et al. [D0 Collaboration].

arXiv:1105.0690 [hep-ex]

Phys. Rev. D 84, 031102 (2011)

1150. "Precise measurement of the top quark mass in the dilepton channel at D0" V. M. Abazov et al. [D0 Collaboration].

arXiv:1105.0320 [hep-ex]

Phys. Rev. Lett. 107, 082004 (2011)

1151. "Measurement of spin correlation in $t \bar{t}$ production using a matrix element approach"

V. M. Abazov et al. [D0 Collaboration].

arXiv:1104.5194 [hep-ex]

Phys. Rev. Lett. 107, 032001 (2011)

1152. "Search for a fourth generation $t^{\prime}$ quark in $p \bar{p}$ collisions at $\sqrt{s}=1.96$ TeV"

V. M. Abazov et al. [D0 Collaboration].

arXiv:1104.4522 [hep-ex]

Phys. Rev. Lett. 107, 082001 (2011)

1153. "Measurement of $\sin ^{2} \theta_{\mathrm{eff}}^{\ell}$ and $Z$-light quark couplings using the forward-backward charge asymmetry in $p \bar{p} \rightarrow Z / \gamma^{*} \rightarrow e^{+} e^{-}$events with $\mathcal{L}=5.0 \mathrm{fb}^{-1}$ at $\sqrt{s}=1.96 \mathrm{TeV}$ " V. M. Abazov et al. [D0 Collaboration].

arXiv:1104.4590 [hep-ex]

Phys. Rev. D 84, 012007 (2011)

1154. "Measurement of the $Z Z$ production cross section in $p \bar{p}$ collisions at $\sqrt{s}=1.96$ TeV"

V. M. Abazov et al. [D0 Collaboration].

arXiv:1104.3078 [hep-ex]

Phys. Rev. D 84, 011103 (2011)

1155. "Determination of the pole and MSbar masses of the top quark from the $t \bar{t}$ cross section"

V. M. Abazov et al. [D0 Collaboration].

arXiv:1104.2887 [hep-ex]

Phys. Lett. B 703, 422 (2011)

1156. "Measurement of three-jet differential cross sections $d \sigma_{3 j \mathrm{jet}} / d M_{3 \mathrm{jet}}$ in $p \bar{p}$ collisions at $\sqrt{s}=1.96 \mathbf{T e V}^{\prime}$

V. M. Abazov et al. [D0 Collaboration].

arXiv:1104.1986 [hep-ex]

Phys. Lett. B 704, 434 (2011)

1157. "Search for flavor changing neutral currents in decays of top quarks"

V. M. Abazov et al. [D0 Collaboration]. 
arXiv:1103.4574 [hep-ex]

Phys. Lett. B 701, 313 (2011)

1158. "Measurement of spin correlation in $t \bar{t}$ production using dilepton final states" V. M. Abazov et al. [D0 Collaboration]. arXiv:1103.1871 [hep-ex]

Phys. Lett. B 702, 16 (2011)

1159. "Search for the Standard Model Higgs Boson in the $H \rightarrow W W \rightarrow \ell \nu q^{\prime} \bar{q}$ Decay Channel"

V. M. Abazov et al. [D0 Collaboration].

arXiv:1101.6079 [hep-ex]

Phys. Rev. Lett. 106, 171802 (2011)

1160. "Azimuthal decorrelations and multiple parton interactions in photon +2 jet and photon +3 jet events in $p \bar{p}$ collisions at $\sqrt{s}=1.96 \mathrm{TeV}^{\prime \prime}$

V. M. Abazov et al. [D0 Collaboration].

arXiv:1101.1509 [hep-ex]

Phys. Rev. D 83, 052008 (2011)

1161. "Measurement of the top quark pair production cross section in the lepton+jets channel in proton-antiproton collisions at $\sqrt{s}=1.96 \mathrm{TeV}^{\prime \prime}$

V. M. Abazov et al. [D0 Collaboration].

arXiv:1101.0124 [hep-ex]

Phys. Rev. D 84, 012008 (2011)

1162. "Measurement of color flow in $t \bar{t}$ events from $p \bar{p}$ collisions at $\sqrt{s}=1.96 \mathrm{TeV}$ "

V. M. Abazov et al. [D0 Collaboration].

arXiv:1101.0648 [hep-ex]

Phys. Rev. D 83, 092002 (2011)

1163. "Search for $W^{\prime}$ - $i$ tb resonances with left- and right-handed couplings to fermions" V. M. Abazov et al. [D0 Collaboration].

arXiv:1101.0806 [hep-ex]

Phys. Lett. B 699, 145 (2011)

1164. "Search for $W H$ associated production in $\mathbf{5 . 3} \mathrm{fb}^{-1}$ of $p \bar{p}$ collisions at the Fermilab Tevatron"

V. M. Abazov et al. [D0 Collaboration].

arXiv:1012.0874 [hep-ex]

Phys. Lett. B 698, 6 (2011)

1165. "Search for resonant $\mathrm{WW}$ and $\mathrm{WZ}$ production in $p \bar{p}$ collisions at ?s $=1.96 \mathrm{TeV}$ " V. M. Abazov et al. [D0 Collaboration].

arXiv:1011.6278 [hep-ex]

Phys. Rev. Lett. 107, 011801 (2011) 
1166. "Measurement of the $\mathrm{W}$ boson helicity in top quark decays using $5.4 \mathrm{fb}^{-1}$ of p$\overline{\mathbf{p}}$ collision data"

V. M. Abazov et al. [D0 Collaboration].

arXiv:1011.6549 [hep-ex]

Phys. Rev. D 83, 032009 (2011)

1167. "Search for neutral Higgs bosons in the multi- $b$-jet topology in $5.2 \mathrm{fb}^{-1}$ of $p \bar{p}$ collisions at $\sqrt{s}=1.96 \mathrm{TeV}^{\prime \prime}$

V. M. Abazov et al. [D0 Collaboration].

arXiv:1011.1931 [hep-ex]

Phys. Lett. B 698, 97 (2011)

1168. "A measurement of the ratio of inclusive cross sections $\sigma(p \bar{p} \rightarrow Z+b$ jet $) / \sigma(p \bar{p} \rightarrow$ $Z+$ jet) at $\sqrt{s}=1.96 \mathbf{T e V}^{\prime \prime}$

V. M. Abazov et al. [D0 Collaboration].

arXiv:1010.6203 [hep-ex]

Phys. Rev. D 83, 031105 (2011)

1169. "Search for single vector-like quarks in $p \bar{p}$ collisions at sqrt(s) $=\mathbf{1 . 9 6}$ TeV"

V. M. Abazov et al. [D0 Collaboration].

arXiv:1010.1466 [hep-ex]

Phys. Rev. Lett. 106, 081801 (2011)

1170. "Precise study of the $Z / \gamma^{*}$ boson transverse momentum distribution in $p \bar{p}$ collisions using a novel technique"

V. M. Abazov et al. [D0 Collaboration].

arXiv:1010.0262 [hep-ex]

Phys. Rev. Lett. 106, 122001 (2011)

1171. "Determination of the width of the top quark"

V. M. Abazov et al. [D0 Collaboration].

arXiv:1009.5686 [hep-ex]

Phys. Rev. Lett. 106, 022001 (2011)

1172. "Search for pair production of the scalar top quark in the electron+muon final state"

V. M. Abazov et al. [D0 Collaboration].

arXiv:1009.5950 [hep-ex]

Phys. Lett. B 696, 321 (2011)

1173. "High mass exclusive diffractive dijet production in $p \bar{p}$ collisions at $\sqrt{\mathbf{s}}=\mathbf{1 . 9 6}$ TeV"

V. M. Abazov et al. [D0 Collaboration].

arXiv:1009.2444 [hep-ex]

Phys. Lett. B 705, 193 (2011)

1174. "Measurement of $t \bar{t}$ production in the tau + jets topology using $p \bar{p}$ collisions at sqrts $=1.96 \mathrm{TeV}^{\prime}$ 
V. M. Abazov et al. [D0 Collaboration].

arXiv:1008.4284 [hep-ex]

Phys. Rev. D 82, 071102 (2010)

1175. "Search for $Z H \rightarrow \ell^{+} \ell^{-} b \bar{b}$ production in $4.2 \mathbf{f b}^{-1}$ of $p \bar{p}$ collisions at $\sqrt{s}=1.96 \mathrm{TeV}$ " V. M. Abazov et al. [D0 Collaboration].

arXiv:1008.3564 [hep-ex]

Phys. Rev. Lett. 105, 251801 (2010)

1176. "Search for New Fermions ('Quirks') at the Fermilab Tevatron Collider"

V. M. Abazov et al. [D0 Collaboration].

arXiv:1008.3547 [hep-ex]

Phys. Rev. Lett. 105, 211803 (2010)

1177. "Search for events with leptonic jets and missing transverse energy in p $\overline{\mathbf{p}}$ collisions at $\sqrt{\mathrm{s}}=1.96 \mathrm{TeV}$ "

V. M. Abazov et al. [D0 Collaboration].

arXiv:1008.3356 [hep-ex]

Phys. Rev. Lett. 105, 211802 (2010)

1178. "Search for a heavy neutral gauge boson in the dielectron channel with 5.4 fb-1 of $p \bar{p}$ collisions at sqrt(s) $=1.96 \mathrm{TeV} "$

V. M. Abazov et al. [D0 Collaboration].

arXiv:1008.2023 [hep-ex]

Phys. Lett. B 695, 88 (2011)

1179. "Search for diphoton events with large missing transverse energy in $6.3 \mathrm{fb}^{-1}$ of $\mathrm{p} \overline{\mathbf{p}}$ collisions at $\sqrt{\mathrm{s}}=1.96 \mathrm{TeV} "$

V. M. Abazov et al. [D0 Collaboration].

arXiv:1008.2133 [hep-ex]

Phys. Rev. Lett. 105, 221802 (2010)

1180. "Search for sneutrino production in emu final states in $\mathbf{5 . 3} \mathrm{fb}^{-1}$ of $p \bar{p}$ collisions at $\operatorname{sqrt}(\mathrm{s})=1.96 \mathrm{TeV}$ "

V. M. Abazov et al. [D0 Collaboration].

arXiv:1007.4835 [hep-ex]

Phys. Rev. Lett. 105, 191802 (2010)

1181. "Evidence for an anomalous like-sign dimuon charge asymmetry"

V. M. Abazov et al. [D0 Collaboration].

arXiv:1007.0395 [hep-ex]

Phys. Rev. Lett. 105, 081801 (2010)

1182. "Search for flavor changing neutral currents via quark-gluon couplings in single top quark production using $2.3 \mathrm{fb}^{-1}$ of $p \bar{p}$ collisions"

V. M. Abazov et al. [D0 Collaboration].

arXiv:1006.3575 [hep-ex]

Phys. Lett. B 693, 81 (2010) 
1183. "Search for the rare decay $B_{s}^{0} \rightarrow m u^{+} m u^{-}$"

V. M. Abazov et al. [D0 Collaboration].

arXiv:1006.3469 [hep-ex]

Phys. Lett. B 693, 539 (2010)

1184. "Measurement of the $W Z \rightarrow \ell \nu \ell \ell$ cross section and limits on anomalous triple gauge couplings in $p \bar{p}$ collisions at $\sqrt{s}=1.96 \mathrm{TeV}^{\prime \prime}$

V. M. Abazov et al. [D0 Collaboration].

arXiv:1006.0761 [hep-ex]

Phys. Lett. B 695, 67 (2011)

1185. "Measurement of the normalized $Z / \gamma^{*}->\mu^{+} \mu^{-}$transverse momentum distribution in $p \bar{p}$ collisions at $\sqrt{s}=1.96 \mathbf{T e V}^{\prime \prime}$

V. M. Abazov et al. [D0 Collaboration].

arXiv:1006.0618 [hep-ex]

Phys. Lett. B 693, 522 (2010)

1186. "Performance of the ATLAS Detector using First Collision Data"

G. Aad et al. [Atlas Collaboration].

arXiv:1005.5254 [hep-ex]

JHEP 1009, 056 (2010)

1187. "Search for scalar bottom quarks and third-generation leptoquarks in p p-bar collisions at $\operatorname{sqrt}(\mathrm{s})=1.96 \mathrm{TeV}$ "

V. M. Abazov et al. [D0 Collaboration].

arXiv:1005.2222 [hep-ex]

Phys. Lett. B 693, 95 (2010)

1188. "Evidence for an anomalous like-sign dimuon charge asymmetry"

V. M. Abazov et al. [D0 Collaboration].

arXiv:1005.2757 [hep-ex]

Phys. Rev. D 82, 032001 (2010)

1189. "Search for Randall-Sundrum gravitons in the dielectron and diphoton final states with $5.4 \mathrm{fb}-1$ of data from $p \bar{p}$ collisions at $\operatorname{sqrt}(\mathrm{s})=1.96 \mathrm{TeV}^{\prime \prime}$

V. M. Abazov et al. [D0 Collaboration].

arXiv:1004.1826 [hep-ex]

Phys. Rev. Lett. 104, 241802 (2010)

1190. "Charged-particle multiplicities in pp interactions at sqrt(s) $=900 \mathrm{GeV}$ measured with the ATLAS detector at the LHC"

G. Aad et al. [ATLAS Collaboration].

arXiv:1003.3124 [hep-ex]

Phys. Lett. B 688, 21 (2010)

1191. "Measurement of direct photon pair production cross sections in $p \bar{p}$ collisions at $\sqrt{s}=1.96 \mathbf{T e V}^{\prime \prime}$

V. M. Abazov et al. [D0 Collaboration]. 
arXiv:1002.4917 [hep-ex]

Phys. Lett. B 690, 108 (2010)

1192. "Measurement of the dijet invariant mass cross section in proton anti-proton collisions at sqrts $=1.96 \mathrm{TeV}$ "

V. M. Abazov et al. [D0 Collaboration].

arXiv:1002.4594 [hep-ex]

Phys. Lett. B 693, 531 (2010)

1193. "b-Jet Identification in the D0 Experiment"

V. M. Abazov et al. [D0 Collaboration].

arXiv:1002.4224 [hep-ex]

Nucl. Instrum. Meth. A 620, 490 (2010)

1194. "Search for Higgs boson production in dilepton and missing energy final states with $5.4 \mathrm{fb}-1$ of p-pbar collisions at $\operatorname{sqrt}(\mathrm{s})=1.96 \mathrm{TeV}$ "

V. M. Abazov et al. [D0 Collaboration].

arXiv:1001.4481 [hep-ex]

Phys. Rev. Lett. 104, 061804 (2010)

1195. "Dependence of the $t \bar{t}$ production cross section on the transverse momentum of the top quark"

V. M. Abazov et al. [D0 Collaboration].

arXiv:1001.1900 [hep-ex]

Phys. Lett. B 693, 515 (2010)

1196. "Search for the standard model Higgs boson in the ZH $-i \mathrm{v}$ v-bar b b-bar channel in $5.2 \mathrm{fb}^{* *}-1$ of $\mathrm{p}$-bar collisions at $\mathrm{s}^{* *}(1 / 2)=1.96-\mathrm{TeV} "$

V. M. Abazov et al. [D0 Collaboration].

arXiv:0912.5285 [hep-ex]

Phys. Rev. Lett. 104, 071801 (2010)

1197. "Double parton interactions in photon $+\mathbf{3}$ jet events in $\mathbf{p}$ p-bar collisions sqrts $=\mathbf{1 . 9 6}$ TeV"

V. M. Abazov et al. [D0 Collaboration].

arXiv:0912.5104 [hep-ex]

Phys. Rev. D 81, 052012 (2010)

1198. "Search for single top quarks in the tau+jets channel using $4.8 \mathrm{fb}^{* *}-1$ of $\mathrm{p}$ p-bar collision data"

V. M. Abazov et al. [D0 Collaboration].

arXiv:0912.1066 [hep-ex]

Phys. Lett. B 690, 5 (2010)

1199. "Search for the associated production of a b quark and a neutral supersymmetric Higgs boson which decays to tau pairs"

V. M. Abazov et al. [D0 Collaboration]. 
arXiv:0912.0968 [hep-ex]

Phys. Rev. Lett. 104, 151801 (2010)

1200. "Search for a resonance decaying into WZ boson pairs in $p \bar{p}$ collisions"

V. M. Abazov et al. [D0 Collaboration].

arXiv:0912.0715 [hep-ex]

Phys. Rev. Lett. 104, 061801 (2010)

1201. "Measurement of the $t \bar{t}$ cross section using high-multiplicity jet events"

V. M. Abazov et al. [D0 Collaboration].

arXiv:0911.4286 [hep-ex]

Phys. Rev. D 82, 032002 (2010)

1202. "Determination of the strong coupling constant from the inclusive jet cross section in $p \bar{p}$ collisions at sqrt(s)=1.96 $\mathrm{TeV}^{\prime \prime}$

V. M. Abazov et al. [D0 Collaboration].

arXiv:0911.2710 [hep-ex]

Phys. Rev. D 80, 111107 (2009)

1203. "Direct measurement of the W boson width"

V. M. Abazov et al. [D0 Collaboration].

arXiv:0909.4814 [hep-ex]

Phys. Rev. Lett. 103, 231802 (2009)

1204. "Search for charged Higgs bosons in top quark decays"

V. M. Abazov et al. [D0 Collaboration].

arXiv:0908.1811 [hep-ex]

Phys. Lett. B 682, 278 (2009)

1205. "Measurement of the W boson mass"

V. M. Abazov et al. [D0 Collaboration].

arXiv:0908.0766 [hep-ex]

Phys. Rev. Lett. 103, 141801 (2009)

1206. "Combined measurements of anomalous charged trilinear gauge-boson couplings from diboson production in $\mathrm{p}$ anti-p collisions at $\mathrm{s}^{* *}(1 / 2)=1.96-\mathrm{TeV}^{\prime \prime}$

V. M. Abazov et al. [D0 Collaboration].

arXiv:0907.4952 [hep-ex]

1207. "Measurement of trilinear gauge boson couplings from $\mathbf{W W}+\mathbf{W Z}-\mathbf{i} \mathbf{l} \mathbf{n u} \mathbf{j} \mathbf{j}$ events in $p$ anti-p collisions at $s^{* *}(1 / 2)=1.96 \mathrm{TeV}^{\prime \prime}$

V. M. Abazov et al. [D0 Collaboration].

arXiv:0907.4398 [hep-ex]

Phys. Rev. D 80, 053012 (2009)

1208. "Measurement of $\mathrm{Z} /$ gamma* + jet $+\mathrm{X}$ angular distributions in $\mathrm{p}$ anti-p collisions at $\mathrm{s}^{* *}(1 / 2)=1.96 . \mathrm{TeV} "$

V. M. Abazov et al. [D0 Collaboration]. 
arXiv:0907.4286 [hep-ex]

Phys. Lett. B 682, 370 (2010)

1209. "Measurement of the t-channel single top quark production cross section" V. M. Abazov et al. [D0 Collaboration]. arXiv:0907.4259 [hep-ex]

Phys. Lett. B 682, 363 (2010)

1210. "A Novel method for modeling the recoil in W boson events at hadron collider" V. M. Abazov et al. [D0 Collaboration]. arXiv:0907.3713 [hep-ex]

Nucl. Instrum. Meth. A 609, 250 (2009)

1211. "Search for pair production of first-generation leptoquarks in $\mathbf{p}$ anti-p collisions at $\mathrm{s}^{* *}(1 / 2)=1.96-\mathrm{TeV} "$

V. M. Abazov et al. [D0 Collaboration].

arXiv:0907.1048 [hep-ex]

Phys. Lett. B 681, 224 (2009)

1212. "Search for charged Higgs bosons in decays of top quarks"

V. M. Abazov et al. [D0 Collaboration].

arXiv:0906.5326 [hep-ex]

Phys. Rev. D 80, 051107 (2009)

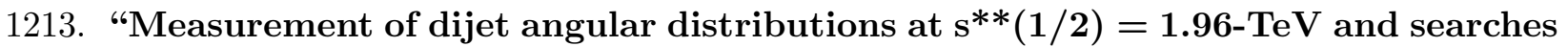
for quark compositeness and extra spatial dimensions"

V. M. Abazov et al. [D0 Collaboration].

arXiv:0906.4819 [hep-ex]

Phys. Rev. Lett. 103, 191803 (2009)

1214. "Search for Resonant Pair Production of long-lived particles decaying to b anti-b in $p$ anti-p collisions at $s^{* *}(1 / 2)=1.96-\mathrm{TeV}^{\prime}$

V. M. Abazov et al. [D0 Collaboration]. arXiv:0906.1787 [hep-ex]

Phys. Rev. Lett. 103, 071801 (2009)

1215. "Direct measurement of the mass difference between top and antitop quarks" V. M. Abazov et al. [D0 Collaboration]. arXiv:0906.1172 [hep-ex]

Phys. Rev. Lett. 103, 132001 (2009)

1216. "Search for squark production in events with jets, hadronically decaying tau leptons and missing transverse energy at $s^{* *}(1 / 2)=1.96-\mathrm{TeV} "$

V. M. Abazov et al. [D0 Collaboration].

arXiv:0905.4086 [hep-ex]

Phys. Lett. B 680, 24 (2009) 
1217. "Search for NMSSM Higgs bosons in the $\mathbf{h}-\mathbf{i}$ aa $-\mathbf{i} \mathbf{m u} \mathbf{m u} \mathbf{m u} \mathbf{m u}, \mathbf{m u} \mathrm{mu}$ tau tau channels using $p$ anti-p collisions at $s^{* *}(1 / 2)=1.96-\mathrm{TeV}^{\prime \prime}$

V. M. Abazov et al. [D0 Collaboration].

arXiv:0905.3381 [hep-ex]

Phys. Rev. Lett. 103, 061801 (2009)

1218. "Search for dark photons from supersymmetric hidden valleys"

V. M. Abazov et al. [D0 Collaboration].

arXiv:0905.1478 [hep-ex]

Phys. Rev. Lett. 103, 081802 (2009)

1219. "Search for CP violation in semileptonic $B_{s}$ decays"

V. M. Abazov et al. [D0 Collaboration].

arXiv:0904.3907 [hep-ex]

Phys. Rev. D 82, 012003 (2010), [Erratum-ibid. D 83, 119901 (2011)]

1220. "Measurement of the top quark mass in final states with two leptons"

V. M. Abazov et al. [D0 Collaboration].

arXiv:0904.3195 [hep-ex]

Phys. Rev. D 80, 092006 (2009)

1221. "Measurement of the WW production cross section with dilepton final states in $\mathrm{p}$ anti-p collisions at $\mathrm{s}^{* *}(1 / 2)=1.96-\mathrm{TeV}$ and limits on anomalous trilinear gauge couplings"

V. M. Abazov et al. [D0 Collaboration].

arXiv:0904.0673 [hep-ex]

Phys. Rev. Lett. 103, 191801 (2009)

1222. "Combination of $\mathrm{t}$ anti-t cross section measurements and constraints on the mass of the top quark and its decays into charged Higgs bosons"

V. M. Abazov et al. [D0 Collaboration].

arXiv:0903.5525 [hep-ex]

Phys. Rev. D 80, 071102 (2009)

1223. "Search for the standard model Higgs boson in tau final states"

V. M. Abazov et al. [D0 Collaboration].

arXiv:0903.4800 [hep-ex]

Phys. Rev. Lett. 102, 251801 (2009)

1224. "Measurements of differential cross sections of $\mathbf{Z} /$ gamma* + jets $+\mathrm{X}$ events in proton anti-proton collisions at $\mathrm{s}^{* *}(1 / 2)=1.96-\mathrm{TeV}^{\prime \prime}$

V. M. Abazov et al. [D0 Collaboration].

arXiv:0903.1748 [hep-ex]

Phys. Lett. B 678, 45 (2009)

1225. "Observation of Single Top Quark Production"

V. M. Abazov et al. [D0 Collaboration]. 
arXiv:0903.0850 [hep-ex]

Phys. Rev. Lett. 103, 092001 (2009)

1226. "Measurement of the $\mathrm{Z}$ gamma - $i$ nu anti-nu gamma cross section and limits on anomalous $\mathrm{Z} \mathrm{Z}$ gamma and $\mathrm{Z}$ gamma gamma couplings in $\mathrm{p}$ anti-p collisions at $\mathrm{s}^{* *}(1 / 2)=1.96-\mathrm{TeV} "$

V. M. Abazov et al. [D0 Collaboration].

arXiv:0902.2157 [hep-ex]

Phys. Rev. Lett. 102, 201802 (2009)

1227. "Measurement of the $t$ anti-t production cross section and top quark mass extraction using dilepton events in p anti-p collisions"

V. M. Abazov et al. [D0 Collaboration].

arXiv:0901.2137 [hep-ex]

Phys. Lett. B 679, 177 (2009)

1228. "Search for Resonant Diphoton Production with the D0 Detector"

V. M. Abazov et al. [D0 Collaboration].

arXiv:0901.1887 [hep-ex]

Phys. Rev. Lett. 102, 231801 (2009)

1229. "Search for admixture of scalar top quarks in the $t$ anti-t lepton+jets final state at $\mathrm{s}^{* *}(1 / 2)=1.96-\mathrm{TeV} "$

V. M. Abazov et al. [D0 Collaboration].

arXiv:0901.1063 [hep-ex]

Phys. Lett. B 674, 4 (2009)

1230. "Measurement of gamma $+\mathbf{b}+\mathbf{X}$ and gamma $+\mathbf{c}+\mathbf{X}$ production cross sections in $p$ anti-p collisions at $s^{* *}(1 / 2)=1.96-\mathrm{TeV}^{\prime}$

V. M. Abazov et al. [D0 Collaboration]. arXiv:0901.0739 [hep-ex]

Phys. Rev. Lett. 102, 192002 (2009)

1231. "Search for associated production of charginos and neutralinos in the trilepton final state using $2.3 \mathrm{fb}^{-1}$ of data"

V. M. Abazov et al. [D0 Collaboration].

arXiv:0901.0646 [hep-ex]

Phys. Lett. B 680, 34 (2009)

1232. "Search for anomalous top quark couplings with the D0 detector"

V. M. Abazov et al. [D0 Collaboration].

arXiv:0901.0151 [hep-ex]

Phys. Rev. Lett. 102, 092002 (2009)

1233. "Evidence for decay $B_{s}^{0} \rightarrow D_{s}^{(*)} D_{s}^{(*)}$ and a measurement of $\Delta \Gamma_{s}^{C P} / \Gamma_{s}$ "

V. M. Abazov et al. [D0 Collaboration].

arXiv:0811.2173 [hep-ex]

Phys. Rev. Lett. 102, 091801 (2009) 
1234. "Search for the lightest scalar top quark in events with two leptons in $p \bar{p}$ collisions at $\sqrt{s}=1.96-\mathrm{TeV}^{\prime \prime}$

V. M. Abazov et al. [D0 Collaboration].

arXiv:0811.0459 [hep-ex]

Phys. Lett. B 675, 289 (2009)

1235. "Search for neutral Higgs bosons at high $\tan ($ beta $)$ in the $b(\mathbf{h} / \mathbf{H} / \mathbf{A})-i$ b tau+ tau- channel"

V. M. Abazov et al. [D0 Collaboration].

arXiv:0811.0024 [hep-ex]

Phys. Rev. Lett. 102, 051804 (2009)

1236. "Evidence of $W W+W Z$ production with lepton + jets final states in protonantiproton collisions at $\sqrt{s}=1.96 \mathrm{TeV}^{\prime \prime}$

V. M. Abazov et al. [D0 Collaboration].

arXiv:0810.3873 [hep-ex]

Phys. Rev. Lett. 102, 161801 (2009)

1237. "Measurement of the angular and lifetime parameters of the decays $B_{d}^{0} \rightarrow J / \psi K^{* 0}$ and $B_{s}^{0} \rightarrow J / \psi \phi "$

V. M. Abazov et al. [D0 Collaboration].

arXiv:0810.0037 [hep-ex]

Phys. Rev. Lett. 102, 032001 (2009)

1238. "Search for Long-Lived Charged Massive Particles with the Do Detector"

V. M. Abazov et al. [D0 Collaboration].

arXiv:0809.4472 [hep-ex]

Phys. Rev. Lett. 102, 161802 (2009)

1239. "The ATLAS Experiment at the CERN Large Hadron Collider"

G. Aad et al. [ATLAS Collaboration].

JINST 3, S08003 (2008).

1240. "Search for Large extra spatial dimensions in the dielectron and diphoton channels in $p \bar{p}$ collisions at $\sqrt{s}=1.96-\mathrm{TeV}^{\prime}$

V. M. Abazov et al. [D0 Collaboration].

arXiv:0809.2813 [hep-ex]

Phys. Rev. Lett. 102, 051601 (2009)

1241. "Observation of the doubly strange $b$ baryon $\Omega_{b}^{-}$"

V. M. Abazov et al. [D0 Collaboration].

arXiv:0808.4142 [hep-ex]

Phys. Rev. Lett. 101, 232002 (2008)

1242. "Search for pair production of second generation scalar leptoquarks"

V. M. Abazov et al. [D0 Collaboration].

arXiv:0808.4023 [hep-ex]

Phys. Lett. B 671, 224 (2009) 
1243. "A Search for associated $W$ and Higgs Boson production in $p \bar{p}$ collisions at $\sqrt{s}=$ 1.96-TeV"

V. M. Abazov et al. [D0 Collaboration].

arXiv:0808.1970 [hep-ex]

Phys. Rev. Lett. 102, 051803 (2009)

1244. "Measurement of $\sigma(p \bar{p} \rightarrow Z+X) \operatorname{Br}\left(Z \rightarrow \tau^{+} \tau^{-}\right)$at $\sqrt{s}=1.96-\mathbf{T e V} "$

V. M. Abazov et al. [D0 Collaboration].

arXiv:0808.1306 [hep-ex]

Phys. Lett. B 670, 292 (2009)

1245. "Measurement of differential $Z / \gamma^{*}+$ jet $+X$ cross sections in $p \bar{p}$ collisions at $\sqrt{s}$ $=1.96-\mathrm{TeV} "$

V. M. Abazov et al. [D0 Collaboration].

arXiv:0808.1296 [hep-ex]

Phys. Lett. B 669, 278 (2008)

1246. "A search for the standard model Higgs boson in the missing energy and acoplanar b-jet topology at $\sqrt{s}=1.96 "$

V. M. Abazov et al. [D0 Collaboration].

arXiv:0808.1266 [hep-ex]

Phys. Rev. Lett. 101, 251802 (2008)

1247. "Observation of $Z Z$ production in $p \bar{p}$ collisions at $\sqrt{s}=1.96-\mathrm{TeV}^{\prime}$

V. M. Abazov et al. [D0 Collaboration].

arXiv:0808.0703 [hep-ex]

Phys. Rev. Lett. 101, 171803 (2008)

1248. "Search for scalar leptoquarks and $T$-odd quarks in the acoplanar jet topology using $2.5 f b^{-1}$ of $p \bar{p}$ collision data at $\sqrt{s}=1.96-\mathrm{TeV}^{\prime \prime}$

V. M. Abazov et al. [D0 Collaboration].

arXiv:0808.0446 [hep-ex]

Phys. Lett. B 668, 357 (2008)

1249. " $Z Z \rightarrow \ell^{+} \ell^{-} \mathbf{v}$ anti-v production in $p \bar{p}$ collisions at $\sqrt{s}=1.96-\mathbf{T e V}$ "

V. M. Abazov et al. [D0 Collaboration].

arXiv:0808.0269 [hep-ex]

Phys. Rev. D 78, 072002 (2008)

1250. "Measurement of the electron charge asymmetry in $p \bar{p} \rightarrow W+X \rightarrow e \nu+X$ events at $\sqrt{s}=1.96-\mathrm{TeV}^{\prime \prime}$

V. M. Abazov et al. [D0 Collaboration].

arXiv:0807.3367 [hep-ex]

Phys. Rev. Lett. 101, 211801 (2008)

1251. "Precise measurement of the top quark mass from lepton+jets events at D0" V. M. Abazov et al. [D0 Collaboration]. 
arXiv:0807.2141 [hep-ex]

Phys. Rev. Lett. 101, 182001 (2008)

1252. "Search for anomalous Wtb couplings in single top quark production"

V. M. Abazov et al. [D0 Collaboration].

arXiv:0807.1692 [hep-ex]

Phys. Rev. Lett. 101, 221801 (2008)

1253. "Search for charged Higgs bosons decaying to top and bottom quarks in $p \bar{p}$ collisions"

V. M. Abazov et al. [D0 Collaboration].

arXiv:0807.0859 [hep-ex]

Phys. Rev. Lett. 102, 191802 (2009)

1254. "Search for third generation scalar leptoquarks decaying into $\tau b$ "

V. M. Abazov et al. [D0 Collaboration].

arXiv:0806.3527 [hep-ex]

Phys. Rev. Lett. 101, 241802 (2008)

1255. "Search for long-lived particles decaying into electron or photon pairs with the D0 detector"

V. M. Abazov et al. [D0 Collaboration].

arXiv:0806.2223 [hep-ex]

Phys. Rev. Lett. 101, 111802 (2008)

1256. "Search for a scalar or vector particle decaying into $Z \gamma$ in $p \bar{p}$ collisions at $\sqrt{s}=1.96$ TeV"

V. M. Abazov et al. [D0 Collaboration].

arXiv:0806.0611 [hep-ex]

Phys. Lett. B 671, 349 (2009)

1257. "Search for neutral Higgs bosons in multi-b-jet events in $p \bar{p}$ collisions at $\sqrt{s}=$ 1.96-TeV"

V. M. Abazov et al. [D0 Collaboration].

arXiv:0805.3556 [hep-ex]

Phys. Rev. Lett. 101, 221802 (2008)

1258. "Measurement of the lifetime of the $B_{c}^{ \pm}$meson in the semileptonic decay channel" V. M. Abazov et al. [D0 Collaboration].

arXiv:0805.2614 [hep-ex]

Phys. Rev. Lett. 102, 092001 (2009)

1259. "Relative rates of $B$ meson decays into $\psi_{2 S}$ and $J / \psi$ mesons"

V. M. Abazov et al. [D0 Collaboration].

arXiv:0805.2576 [hep-ex]

Phys. Rev. D 79, 111102 (2009) 
1260. "Search for Higgs bosons decaying to $\tau$ pairs in $p \bar{p}$ collisions with the Do detector" V. M. Abazov et al. [D0 Collaboration].

arXiv:0805.2491 [hep-ex]

Phys. Rev. Lett. 101, 071804 (2008)

1261. "Search for $t \bar{t}$ resonances in the lepton plus jets final state in $p \bar{p}$ collisions at $\sqrt{s}$ $=1.96 \mathrm{TeV}^{\prime}$

V. M. Abazov et al. [D0 Collaboration].

arXiv:0804.3664 [hep-ex]

Phys. Lett. B 668, 98 (2008)

1262. "Measurement of the forward-backward charge asymmetry and extraction of $\sin ^{* * 2}$ Theta $(\mathrm{W})(\mathrm{eff})$ in $\mathrm{p}$ anti-p to $\mathrm{Z} /$ gamma ${ }^{*}+\mathrm{X}$ to e $+\mathrm{e}-+\mathrm{X}$ events produced at $\mathrm{s}^{* *}(1 / 2)=1.96 \mathrm{TeV}^{\prime \prime}$

V. M. Abazov et al. [D0 Collaboration].

arXiv:0804.3220 [hep-ex]

Phys. Rev. Lett. 101, 191801 (2008)

1263. "Measurement of the polarization of the $v_{1 S}$ and $v_{2 S}$ states in $p \bar{p}$ collisions at $\sqrt{s}$

$=1.96 \mathrm{TeV} "$

V. M. Abazov et al. [D0 Collaboration].

arXiv:0804.2799 [hep-ex]

Phys. Rev. Lett. 101, 182004 (2008)

1264. "Measurement of the differential cross-section for the production of an isolated photon with associated jet in $p \bar{p}$ collisions at $\sqrt{s}=1.96-\mathrm{TeV}^{\prime \prime}$

V. M. Abazov et al. [D0 Collaboration].

arXiv:0804.1107 [hep-ex]

Phys. Lett. B 666, 435 (2008)

1265. "Search for $W^{\prime}$ Boson Resonances Decaying to a Top Quark and a Bottom Quark" V. M. Abazov et al. [D0 Collaboration].

arXiv:0803.3256 [hep-ex]

Phys. Rev. Lett. 100, 211803 (2008)

1266. "Measurement of the $t \bar{t}$ production cross section in $p \bar{p}$ collisions at $\sqrt{s}=1.96$ TeV"

V. M. Abazov et al. [D0 Collaboration].

arXiv:0803.2779 [hep-ex]

Phys. Rev. Lett. 100, 192004 (2008)

1267. "Search for scalar top quarks in the acoplanar charm jets and missing transverse energy final state in $p \bar{p}$ collisions at $\sqrt{s}=1.96-\mathrm{TeV}^{\prime \prime}$

V. M. Abazov et al. [D0 Collaboration].

arXiv:0803.2263 [hep-ex]

Phys. Lett. B 665, 1 (2008) 
1268. "Measurement of the ratio of the $p \bar{p} \rightarrow W^{+} c^{-}$jet cross section to the inclusive $p \bar{p} \rightarrow W+$ jets cross section"

V. M. Abazov et al. [D0 Collaboration].

arXiv:0803.2259 [hep-ex]

Phys. Lett. B 666, 23 (2008)

1269. "Search for large extra dimensions via single photon plus missing energy final states at $\sqrt{s}=1.96-\mathrm{TeV}^{\prime}$

V. M. Abazov et al. [D0 Collaboration].

arXiv:0803.2137 [hep-ex]

Phys. Rev. Lett. 101, 011601 (2008)

1270. "Search for pair production of doubly-charged Higgs bosons in the $H^{++} H^{--} \rightarrow$ $\mu^{+} \mu^{+} \mu^{-} \mu^{-}$final state at Do"

V. M. Abazov et al. [D0 Collaboration].

arXiv:0803.1534 [hep-ex]

Phys. Rev. Lett. 101, 071803 (2008)

1271. "Search for decay of a fermiophobic Higgs boson $h(f) \rightarrow \gamma \gamma$ with the Do detector at $\sqrt{s}=1.96-\mathrm{TeV}^{\prime \prime}$

V. M. Abazov et al. [D0 Collaboration].

arXiv:0803.1514 [hep-ex]

Phys. Rev. Lett. 101, 051801 (2008)

1272. "Evidence for production of single top quarks"

V. M. Abazov et al. [D0 Collaboration].

arXiv:0803.0739 [hep-ex]

Phys. Rev. D 78, 012005 (2008)

1273. "First study of the radiation-amplitude zero in $W \gamma$ production and limits on anomalous $W W \gamma$ couplings at $\sqrt{s}=1.96-\mathbf{T e V} "$

V. M. Abazov et al. [D0 Collaboration].

arXiv:0803.0030 [hep-ex]

Phys. Rev. Lett. 100, 241805 (2008)

1274. "Observation of the $B_{c}$ Meson in the Exclusive Decay $B_{c} \rightarrow J / \psi \pi$ "

V. M. Abazov et al. [D0 Collaboration].

arXiv:0802.4258 [hep-ex]

Phys. Rev. Lett. 101, 012001 (2008)

1275. "Study of direct CP violation in $B^{ \pm} \rightarrow J / \psi K^{ \pm}\left(\pi^{ \pm}\right)$decays"

V. M. Abazov et al. [D0 Collaboration].

arXiv:0802.3299 [hep-ex]

Phys. Rev. Lett. 100, 211802 (2008)

1276. "Measurement of the inclusive jet cross-section in $p \bar{p}$ collisions at $s^{91 / 2)}=\mathbf{1 . 9 6}$ TeV"

V. M. Abazov et al. [D0 Collaboration]. 
arXiv:0802.2400 [hep-ex]

Phys. Rev. Lett. 101, 062001 (2008)

1277. "Measurement of $B_{s}^{0}$ mixing parameters from the flavor-tagged decay $B_{s}^{0} \rightarrow J / \psi \phi "$ V. M. Abazov et al. [D0 Collaboration].

arXiv:0802.2255 [hep-ex]

Phys. Rev. Lett. 101, 241801 (2008)

1278. "Simultaneous measurement of the ratio $\mathbf{B}(t \rightarrow W b) / \mathbf{B}(t \rightarrow W q)$ and the top quark pair production cross section with the Do detector at $\sqrt{s}=1.96-\mathrm{TeV}$ "

V. M. Abazov et al. [D0 Collaboration].

arXiv:0801.1326 [hep-ex]

Phys. Rev. Lett. 100, 192003 (2008)

1279. "Search for excited electrons in $p \bar{p}$ collisions at $\sqrt{s}=1.96-\mathbf{T e V}$ "

V. M. Abazov et al. [D0 Collaboration].

arXiv:0801.0877 [hep-ex]

Phys. Rev. D 77, 091102 (2008)

1280. "Search for squarks and gluinos in events with jets and missing transverse energy using $2.1 f b^{-1}$ of $p \bar{p}$ collision data at $\sqrt{s}=1.96-\mathrm{TeV}$ "

V. M. Abazov et al. [D0 Collaboration].

arXiv:0712.3805 [hep-ex]

Phys. Lett. B 660, 449 (2008)

1281. "Measurement of the $B_{s}^{0}$ semileptonic branching ratio to an orbitally excited $D_{s}$ state, $\operatorname{Br}\left(B_{s}^{0} \rightarrow D_{s 1}^{-}(2536) \mu^{+} \nu X\right) "$

V. M. Abazov et al. [D0 Collaboration].

arXiv:0712.3789 [hep-ex]

Phys. Rev. Lett. 102, 051801 (2009)

1282. "First measurement of the forward-backward charge asymmetry in top quark pair production"

V. M. Abazov et al. [D0 Collaboration].

arXiv:0712.0851 [hep-ex]

Phys. Rev. Lett. 100, 142002 (2008)

1283. "Measurement of the shape of the boson transverse momentum distribution in $p \bar{p} \rightarrow Z / \gamma^{*} \rightarrow e^{+} e^{-}+X$ events produced at $\sqrt{s}=1.96-\mathbf{T e V} "$

V. M. Abazov et al. [D0 Collaboration].

arXiv:0712.0803 [hep-ex]

Phys. Rev. Lett. 100, 102002 (2008)

1284. "Search for $Z Z$ and $Z \gamma^{*}$ production in $p \bar{p}$ collisions at $\sqrt{s}=1.96 \mathrm{TeV}$ and limits on anomalous $Z Z Z$ and $Z Z \gamma^{*}$ couplings"

V. M. Abazov et al. [D0 Collaboration].

arXiv:0712.0599 [hep-ex]

Phys. Rev. Lett. 100, 131801 (2008) 
1285. "A Combined search for the standard model Higgs boson at $\sqrt{s}=1.96-\mathrm{TeV} "$ V. M. Abazov et al. [D0 Collaboration]. arXiv:0712.0598 [hep-ex]

Phys. Lett. B 663, 26 (2008)

1286. "Search for Scalar Neutrino Superpartners in $e+\mu$ Final States in $p \bar{p}$ Collisions at $\sqrt{s}=1.96-\mathrm{TeV}^{\prime}$

V. M. Abazov et al. [D0 Collaboration].

arXiv:0711.3207 [hep-ex]

Phys. Rev. Lett. 100, 241803 (2008)

1287. "Model-independent measurement of the $W$ boson helicity in top quark decays at D0"

V. M. Abazov et al. [D0 Collaboration].

arXiv:0711.0032 [hep-ex]

Phys. Rev. Lett. 100, 062004 (2008)

1288. "Observation and properties of the orbitally excited B*(s2) meson"

V. M. Abazov et al. [D0 Collaboration].

arXiv:0711.0319 [hep-ex]

Phys. Rev. Lett. 100, 082002 (2008)

1289. "Search for supersymmetry in di-photon final states at $\sqrt{s}=1.96-\mathrm{TeV}$ "

V. M. Abazov et al. [D0 Collaboration].

arXiv:0710.3946 [hep-ex]

Phys. Lett. B 659, 856 (2008)

1290. "Search for Randall-Sundrum gravitons with $1 \mathrm{fb}^{-1}$ of data from $p \bar{p}$ collisions at $\sqrt{s}=1.96-\mathrm{TeV}^{\prime}$

V. M. Abazov et al. [D0 Collaboration].

arXiv:0710.3338 [hep-ex]

Phys. Rev. Lett. 100, 091802 (2008)

1291. "Search for $W^{\prime}$ bosons decaying to an electron and a neutrino with the D0 detector"

V. M. Abazov et al. [D0 Collaboration].

arXiv:0710.2966 [hep-ex]

Phys. Rev. Lett. 100, 031804 (2008)

1292. "Measurement of the muon charge asymmetry from $W$ boson decays"

V. M. Abazov et al. [D0 Collaboration].

arXiv:0709.4254 [hep-ex]

Phys. Rev. D 77, 011106 (2008)

1293. "Measurement of the $p \bar{p} \rightarrow W Z+X$ cross-section at $\sqrt{s}=1.96-\mathrm{TeV}$ and limits on WWZ trilinear gauge couplings"

V. M. Abazov et al. [D0 Collaboration]. 
arXiv:0709.2917 [hep-ex]

Phys. Rev. D 76, 111104 (2007)

1294. "Search for flavor-changing-neutral-current $D$ meson decays"

V. M. Abazov et al. [D0 Collaboration].

arXiv:0708.2094 [hep-ex]

Phys. Rev. Lett. 100, 101801 (2008)

1295. "Search for $B_{s} \rightarrow \mu^{+} \mu^{-}$at Do"

V. M. Abazov et al. [D0 Collaboration].

arXiv:0707.3997 [hep-ex]

Phys. Rev. D 76, 092001 (2007)

1296. "Search for the lightest scalar top quark in events with two leptons in $p \bar{p}$ collisions at $\sqrt{s}=1.96-\mathrm{TeV} "$

V. M. Abazov et al. [D0 Collaboration].

arXiv:0707.2864 [hep-ex]

Phys. Lett. B 659, 500 (2008)

1297. "Measurement of the $\Lambda_{b}^{0}$ lifetime using semileptonic decays"

V. M. Abazov et al. [D0 Collaboration].

arXiv:0706.2358 [hep-ex]

Phys. Rev. Lett. 99, 182001 (2007)

1298. "Direct observation of the strange $b$ baryon $\Xi_{b}^{-}$"

V. M. Abazov et al. [D0 Collaboration].

arXiv:0706.1690 [hep-ex]

Phys. Rev. Lett. 99, 052001 (2007)

1299. "Measurement of the $t \bar{t}$ production cross-section in $p \bar{p}$ collisions using dilepton events"

V. M. Abazov et al. [D0 Collaboration].

arXiv:0706.0458 [hep-ex]

Phys. Rev. D 76, 052006 (2007)

1300. "Observation and Properties of $L=1 B_{1}$ and $B_{2}^{*}$ Mesons"

V. M. Abazov et al. [D0 Collaboration].

arXiv:0705.3229 [hep-ex]

Phys. Rev. Lett. 99, 172001 (2007)

1301. "Measurement of the $t \bar{t}$ production cross section in $p \bar{p}$ collisions at $\sqrt{s}=1.96-\mathrm{TeV}$ using kinematic characteristics of lepton + jets events"

V. M. Abazov et al. [D0 Collaboration].

arXiv:0705.2788 [hep-ex]

Phys. Rev. D 76, 092007 (2007)

1302. " $Z \gamma$ production and limits on anomalous $Z Z \gamma$ and $Z \gamma \gamma$ couplings in $p \bar{p}$ collisions at $\sqrt{s}=1.96-\mathrm{TeV}^{\prime \prime}$ 
V. M. Abazov et al. [D0 Collaboration].

arXiv:0705.1550 [hep-ex]

Phys. Lett. B 653, 378 (2007)

1303. "Search for third-generation leptoquarks in $p \bar{p}$ collisions at $\sqrt{s}=1.96-\mathrm{TeV}$ "

V. M. Abazov et al. [D0 Collaboration].

arXiv:0705.0812 [hep-ex]

Phys. Rev. Lett. 99, 061801 (2007)

1304. "Search for stopped gluinos from $p \bar{p}$ collisions at $\sqrt{s}=1.96-\mathbf{T e V}$ "

V. M. Abazov et al. [D0 Collaboration].

arXiv:0705.0306 [hep-ex]

Phys. Rev. Lett. 99, 131801 (2007)

1305. "Measurement of the $\Lambda_{b}$ lifetime in the exclusive decay $\Lambda_{b} \rightarrow J / \psi \Lambda$ "

V. M. Abazov et al. [D0 Collaboration].

arXiv:0704.3909 [hep-ex]

Phys. Rev. Lett. 99, 142001 (2007)

1306. "Search for a Higgs boson produced in association with a $Z$ boson in $p \bar{p}$ collisions" V. M. Abazov et al. [D0 Collaboration].

arXiv:0704.2000 [hep-ex]

Phys. Lett. B 655, 209 (2007)

1307. "Measurement of the branching fraction $\operatorname{Br}\left(B^{0}(s) \rightarrow D_{s}^{(*)} D_{s}^{(*)}\right)$ "

V. M. Abazov et al. [D0 Collaboration].

hep-ex/0702049 [HEP-EX]

Phys. Rev. Lett. 99, 241801 (2007)

1308. "Combined $D^{0}$ measurements constraining the CP-violating phase and width difference in the $B_{s}^{0}$ system"

V. M. Abazov et al. [D0 Collaboration].

hep-ex/0702030 [HEP-EX]

Phys. Rev. D 76, 057101 (2007)

1309. "Measurement of the shape of the boson rapidity distribution for $p \bar{p} \rightarrow Z /$ gamma* $^{*}$ $e^{+} e^{-}+X$ events produced at $\sqrt{s}$ of 1.96-TeV"

V. M. Abazov et al. [D0 Collaboration].

hep-ex/0702025 [HEP-EX]

Phys. Rev. D 76, 012003 (2007)

1310. "Measurement of the top quark mass in the lepton + jets channel using the Ideogram method"

V. M. Abazov et al. [D0 Collaboration].

hep-ex/0702018 [HEP-EX]

Phys. Rev. D 75, 092001 (2007) 
1311. "Search for production of single top quarks via tcg and tug flavor- changing neutral current couplings"

V. M. Abazov et al. [D0 Collaboration]. hep-ex/0702005 [HEP-EX], arXiv:0801.2556 [HEP-EX]

Phys. Rev. Lett. 99, 191802 (2007)

1312. "Lifetime difference and CP-violating phase in the $B_{s}^{0}$ system"

V. M. Abazov et al. [D0 Collaboration].

hep-ex/0701012

Phys. Rev. Lett. 98, 121801 (2007)

1313. "Measurement of the charge asymmetry in semileptonic $B_{s}$ decays"

V. M. Abazov et al. [D0 Collaboration].

hep-ex/0701007

Phys. Rev. Lett. 98, 151801 (2007)

1314. "Evidence for production of single top quarks and first direct measurement of - Vtb-"

V. M. Abazov et al. [D0 Collaboration].

hep-ex/0612052

Phys. Rev. Lett. 98, 181802 (2007)

1315. "Measurement of the $p \bar{p} \rightarrow t \bar{t}$ production cross section at $\sqrt{s}=1.96-\mathrm{TeV}$ in the fully hadronic decay channel."

V. M. Abazov et al. [D0 Collaboration].

hep-ex/0612040

Phys. Rev. D 76, 072007 (2007)

1316. "Search for techniparticles in e+jets events at D0"

V. M. Abazov et al. [D0 Collaboration].

hep-ex/0612013

Phys. Rev. Lett. 98, 221801 (2007)

1317. "Search for single production of scalar leptoquarks in $\mathbf{p}$ anti-p collisions decaying into muons and quarks with the Do detector"

V. M. Abazov et al. [D0 Collaboration].

hep-ex/0612012

Phys. Lett. B 647, 74 (2007)

1318. "Search for the pair production of scalar top quarks in the acoplanar charm jet final state in $p$ anti-p collisions at $s^{* *}(1 / 2)=1.96-\mathrm{TeV}^{\prime \prime}$

V. M. Abazov et al. [D0 Collaboration].

hep-ex/0611003

Phys. Lett. B 645, 119 (2007)

1319. "Measurement of the $\mathrm{t}$ anti-t production cross section in $\mathrm{p}$ anti-p collisions at $s^{* *}(1 / 2)=1.96-\mathrm{TeV}$ using secondary vertex $b$ tagging"

V. M. Abazov et al. [D0 Collaboration]. 
hep-ex/0611002

Phys. Rev. D 74, 112004 (2006)

1320. "Measurement of the top quark mass in the dilepton channel"

V. M. Abazov et al. [D0 Collaboration].

hep-ex/0609056

Phys. Lett. B 655, 7 (2007)

1321. "Measurement of the top quark mass in the lepton+jets final state with the matrix element method"

V. M. Abazov et al. [D0 Collaboration].

hep-ex/0609053

Phys. Rev. D 74, 092005 (2006)

1322. "Measurement of the W boson helicity in top quark decay at D0"

V. M. Abazov et al. [D0 Collaboration].

hep-ex/0609045

Phys. Rev. D 75, 031102 (2007)

1323. "Measurement of $B_{d}$ mixing using opposite-side flavor tagging"

V. M. Abazov et al. [D0 Collaboration].

hep-ex/0609034

Phys. Rev. D 74, 112002 (2006)

1324. "Measurement of the CP-violation parameter of B0 mixing and decay with p anti-p - i mu mu X data"

V. M. Abazov et al. [D0 Collaboration].

hep-ex/0609014

Phys. Rev. D 74, 092001 (2006)

1325. "Measurement of the ratios of the $\mathrm{Z} /$ gamma* $+i=\mathbf{n}$ jet production cross sections to the total inclusive $\mathrm{Z} /$ gamma* $^{*}$ cross section in $\mathrm{p}$ anti-p collisions at $\mathrm{s}^{* *}(1 / 2)=1.96-\mathrm{TeV}^{\prime \prime}$

V. M. Abazov et al. [D0 Collaboration].

hep-ex/0608052

Phys. Lett. B 658, 112 (2008)

1326. "Experimental discrimination between charge 2e/3 top quark and charge $4 \mathrm{e} / 3$ exotic quark production scenarios"

V. M. Abazov et al. [D0 Collaboration].

hep-ex/0608044

Phys. Rev. Lett. 98, 041801 (2007)

1327. "Search for pair production of scalar bottom quarks in $p \bar{p}$ collisions at $\sqrt{s}=1.96$ TeV"

V. M. Abazov et al. [D0 Collaboration].

hep-ex/0608013

Phys. Rev. Lett. 97, 171806 (2006) 
1328. "Limits on anomalous trilinear gauge couplings from $W W \rightarrow e^{+} e^{-}, W W \rightarrow e^{ \pm} \mu^{\mp}$, and $W W \rightarrow \mu^{+} \mu^{-}$events from $p \bar{p}$ collisions at $\sqrt{s}=1.96-\mathbf{T e V}$ "

V. M. Abazov et al. [D0 Collaboration].

hep-ex/0608011

Phys. Rev. D 74, 057101 (2006), [Erratum-ibid. D 74, 059904 (2006)]

1329. "Search for $W^{\prime}$ boson production in the top quark decay channel"

V. M. Abazov et al. [D0 Collaboration].

hep-ex/0607102

Phys. Lett. B 641, 423 (2006)

1330. "Search for associated Higgs boson production $\mathbf{W H} \rightarrow \mathbf{W} \mathbf{W} \mathbf{W}^{*} \rightarrow \ell^{ \pm} \nu \ell^{\prime}+-\nu^{\prime}+$ $X$ in $p \bar{p}$ collisions at $\sqrt{s}=1.96-\mathrm{TeV} "$

V. M. Abazov et al. [D0 Collaboration].

hep-ex/0607032

Phys. Rev. Lett. 97, 151804 (2006)

1331. "Search for neutral, long-lived particles decaying into two muons in $p \bar{p}$ collisions at $\sqrt{s}=1.96-\mathrm{TeV}^{\prime \prime}$

V. M. Abazov et al. [D0 Collaboration].

hep-ex/0607028

Phys. Rev. Lett. 97, 161802 (2006)

1332. "Search for the Standard Model Higgs Boson in the $p \bar{p} \rightarrow \mathbf{Z H} \rightarrow \nu \bar{\nu} b \bar{b}$ channel"

V. M. Abazov et al. [D0 Collaboration].

hep-ex/0607022

Phys. Rev. Lett. 97, 161803 (2006)

1333. "Search for scalar leptoquarks in the acoplanar jet topology in $p \bar{p}$ collisions at $\sqrt{s}$ $=1.96-\mathrm{TeV}^{\prime \prime}$

V. M. Abazov et al. [D0 Collaboration].

hep-ex/0607009

Phys. Lett. B 640, 230 (2006)

1334. "Search for a heavy resonance decaying into a $Z+$ jet final state in $p \bar{p}$ collisions at $\sqrt{s}=1.96-\mathrm{TeV}$ using the Do detector"

V. M. Abazov et al. [D0 Collaboration].

hep-ex/0606018

Phys. Rev. D 74, 011104 (2006)

1335. "Search for particles decaying into a $Z$ boson and a photon in $p \bar{p}$ collisions at $\sqrt{s}$ $=1.96 \mathrm{TeV}$ "

V. M. Abazov et al. [D0 Collaboration].

hep-ex/0605064

Phys. Lett. B 641, 415 (2006), [Erratum-ibid. B 670, 455 (2009)]

1336. "Search for resonant second generation slepton production at the Tevatron" V. M. Abazov et al. [D0 Collaboration]. 
hep-ex/0605010

Phys. Rev. Lett. 97, 111801 (2006)

1337. "Search for neutral Higgs bosons decaying to $\tau$ pairs in $p \bar{p}$ collisions at $\sqrt{s}=$ 1.96-TeV"

V. M. Abazov et al. [D0 Collaboration].

hep-ex/0605009

Phys. Rev. Lett. 97, 121802 (2006)

1338. "Search for R-parity violating supersymmetry via the LL anti-E couplings $\lambda_{121}$, $\lambda_{122}$ or $\lambda_{133}$ in $p \bar{p}$ collisions at $\sqrt{s}=\mathbf{1 . 9 6 - T e V "}$

V. M. Abazov et al. [D0 Collaboration].

hep-ex/0605005

Phys. Lett. B 638, 441 (2006)

1339. "A Precise measurement of the $B_{s}^{0}$ lifetime"

V. M. Abazov et al. [D0 Collaboration].

hep-ex/0604046

Phys. Rev. Lett. 97, 241801 (2006)

1340. "Search for excited muons in $p \bar{p}$ collisions at $\sqrt{s}=1.96-\mathrm{TeV}$ "

V. M. Abazov et al. [D0 Collaboration].

hep-ex/0604040

Phys. Rev. D 73, 111102 (2006)

1341. "Search for squarks and gluinos in events with jets and missing transverse energy in $p \bar{p}$ collisions at $\sqrt{s}=1.96-\mathrm{TeV}^{\prime \prime}$

V. M. Abazov et al. [D0 Collaboration].

hep-ex/0604029

Phys. Lett. B 638, 119 (2006)

1342. "Multivariate searches for single top quark production with the Do detector" V. M. Abazov et al. [D0 Collaboration].

hep-ex/0604020

Phys. Rev. D 75, 092007 (2007)

1343. "Search for the rare decay $B_{s}^{0} \rightarrow \phi \mu^{+} \mu^{-}$with the D0 detector"

V. M. Abazov et al. [D0 Collaboration].

hep-ex/0604015

Phys. Rev. D 74, 031107 (2006)

1344. "First direct two-sided bound on the $B_{s}^{0}$ oscillation frequency"

V. M. Abazov et al. [D0 Collaboration].

hep-ex/0603029

Phys. Rev. Lett. 97, 021802 (2006)

1345. "Measurement of $\mathbf{B}(t \rightarrow \mathbf{W b}) / \mathbf{B}(t \rightarrow \mathbf{W q})$ at $\sqrt{s}=1.96-\mathbf{T e V}$ "

V. M. Abazov et al. [D0 Collaboration]. 
hep-ex/0603002

Phys. Lett. B 639, 616 (2006)

1346. "Search for pair production of second generation scalar leptoquarks in p anti-p collisions at $\sqrt{s}=1.96-\mathrm{TeV}$."

V. M. Abazov et al. [D0 Collaboration].

hep-ex/0601047

Phys. Lett. B 636, 183 (2006)

1347. "Measurement of the isolated photon cross section in $p \bar{p}$ collisions at $\sqrt{s}=1.96$ TeV"

V. M. Abazov et al. [D0 Collaboration].

hep-ex/0511054

Phys. Lett. B 639, 151 (2006), [Erratum-ibid. B 658, 285 (2008)]

1348. "Construction, assembly and tests of the ATLAS electromagnetic barrel calorimeter"

B. Aubert et al. [ATLAS Electromagnetic Barrel Liquid Argon Calorimeter Group Collaboration].

Nucl. Instrum. Meth. A 558, 388 (2006).

1349. "Search for the Higgs boson in $H \rightarrow W W^{(*)}$ decays in $p \bar{p}$ collisions at $\sqrt{s}=1.96$ TeV"

V. M. Abazov et al. [D0 Collaboration].

hep-ex/0508054

Phys. Rev. Lett. 96, 011801 (2006)

1350. "The Upgraded Do detector"

V. M. Abazov et al. [D0 Collaboration].

physics/0507191 [physics.ins-det]

Nucl. Instrum. Meth. A 565, 463 (2006)

1351. "Measurement of the lifetime difference in the B0(s) system"

V. M. Abazov et al. [D0 Collaboration].

hep-ex/0507084

Phys. Rev. Lett. 95, 171801 (2005)

1352. "Measurement of semileptonic branching fractions of $B$ mesons to narrow $D^{* *}$ states"

V. M. Abazov et al. [D0 Collaboration].

hep-ex/0507046

Phys. Rev. Lett. 95, 171803 (2005)

1353. "Search for large extra spatial dimensions in dimuon production at D0"

V. M. Abazov et al. [D0 Collaboration].

hep-ex/0506063

Phys. Rev. Lett. 95, 161602 (2005) 
1354. "Nuclear effects in high-p(T) production of direct photons and neutral mesons" L. Apanasevich et al. [Fermilab E706 Collaboration].

hep-ex/0506003

Phys. Rev. D 72, 032003 (2005)

1355. "Measurement of the $t \bar{t}$ production cross section in $p \bar{p}$ collisions at $\sqrt{s}=1.96-\mathrm{TeV}$ in dilepton final states"

V. M. Abazov et al. [D0 Collaboration].

hep-ex/0505082

Phys. Lett. B 626, 55 (2005)

1356. "Search for single top quark production in $p \bar{p}$ collisions at $\sqrt{s}=1.96-\mathrm{TeV}$ "

V. M. Abazov et al. [D0 Collaboration].

hep-ex/0505063

Phys. Lett. B 622, 265 (2005)

1357. "Search for right-handed $W$ bosons in top quark decay"

V. M. Abazov et al. [D0 Collaboration].

hep-ex/0505031

Phys. Rev. D 72, 011104 (2005)

1358. "Search for Randall-Sundrum gravitons in dilepton and diphoton final states"

V. M. Abazov et al. [D0 Collaboration].

hep-ex/0505018

Phys. Rev. Lett. 95, 091801 (2005)

1359. "Measurement of the $t \bar{t}$ production cross section in $p \bar{p}$ collisions at $\sqrt{s}=1.96-\mathbf{T e V}$ using lepton + jets events with lifetime $b^{-}$tagging"

V. M. Abazov et al. [D0 Collaboration].

hep-ex/0504058

Phys. Lett. B 626, 35 (2005)

1360. "Measurement of the $t \bar{t}$ production cross section in $p \bar{p}$ collisions at $\sqrt{s}=\mathbf{1 . 9 6}=$ TeV using kinematic characteristics of lepton + jets events"

V. M. Abazov et al. [D0 Collaboration].

hep-ex/0504043

Phys. Lett. B 626, 45 (2005)

1361. "Search for supersymmetry via associated production of charginos and neutralinos in final states with three leptons"

V. M. Abazov et al. [D0 Collaboration].

hep-ex/0504032

Phys. Rev. Lett. 95, 151805 (2005)

1362. "Production of $\mathrm{WZ}$ events in $p \bar{p}$ collisions at $\sqrt{s}=1.96-\mathrm{TeV}$ and limits on anomalous WWZ couplings"

V. M. Abazov et al. [D0 Collaboration]. 
hep-ex/0504019

Phys. Rev. Lett. 95, 141802 (2005)

1363. "Search for neutral supersymmetric Higgs bosons in multijet events at $\sqrt{s}=$ 1.96-TeV"

V. M. Abazov et al. [D0 Collaboration].

hep-ex/0504018

Phys. Rev. Lett. 95, 151801 (2005)

1364. "Measurement of the $p-\bar{p} \rightarrow W \gamma+X$ cross section at $\sqrt{s}=1.96-\mathrm{TeV}$ and $W W \gamma$ anomalous coupling limits"

V. M. Abazov et al. [D0 Collaboration].

hep-ex/0503048

Phys. Rev. D 71, 091108 (2005)

1365. "Study of $Z \gamma$ events and limits on anomalous $Z Z \gamma$ and $Z \gamma \gamma$ couplings in $p \bar{p}$ collisions at $\sqrt{s}=1.96-\mathrm{TeV}^{\prime}$

V. M. Abazov et al. [D0 Collaboration].

hep-ex/0502036

Phys. Rev. Lett. 95, 051802 (2005)

1366. "Measurement of inclusive differential cross sections for $v_{1 S}$ production in $p \bar{p}$ collisions at $\sqrt{s}=1.96-\mathrm{TeV}^{\prime \prime}$

V. M. Abazov et al. [D0 Collaboration].

hep-ex/0502030

Phys. Rev. Lett. 94, 232001 (2005), [Erratum-ibid. 100, 049902 (2008)]

1367. "Search for first-generation scalar leptoquarks in $p \bar{p}$ collisions at $\sqrt{s}=\mathbf{1 . 9 6 - T e V " ~}$ V. M. Abazov et al. [D0 Collaboration].

hep-ex/0412029

Phys. Rev. D 71, 071104 (2005)

1368. "First measurement of $\sigma(p \bar{p} \rightarrow Z)$. $\mathrm{Br}(Z \rightarrow \tau \tau)$ at $\sqrt{s}=1.96-\mathbf{T e V}$ "

V. M. Abazov et al. [D0 Collaboration].

hep-ex/0412020, hep-ex/

Phys. Rev. D 71, 072004 (2005), [Erratum-ibid. D 77, 039901 (2008)]

1369. "A Search for anomalous heavy-flavor quark production in association with $W$ bosons"

V. M. Abazov et al. [D0 Collaboration].

hep-ex/0411084

Phys. Rev. Lett. 94, 152002 (2005)

1370. "Measurement of the top quark mass in all-jet events"

V. M. Abazov et al. [D0 Collaboration].

hep-ex/0410086

Phys. Lett. B 606, 25 (2005) 
1371. "A measurement of the ratio of inclusive cross sections $\sigma(p p \rightarrow Z+b-$ jet $) / \sigma(p p \rightarrow$ $Z+$ jet) at $\sqrt{s}=1.96 \mathbf{T e V}^{\prime \prime}$

V. M. Abazov et al. [D0 Collaboration].

hep-ex/0410078

Phys. Rev. Lett. 94, 161801 (2005)

1372. "Measurement of the $W W$ production cross section in $p \bar{p}$ collisions at $\sqrt{s}=1.96$ TeV"

V. M. Abazov et al. [D0 Collaboration].

hep-ex/0410066

Phys. Rev. Lett. 94, 151801 (2005), [Erratum-ibid. 100, 139901 (2008)]

1373. "A search for $W b \bar{b}$ and $W H$ production in $p \bar{p}$ collisions at $\sqrt{s}=1.96 \mathbf{T e V}$ "

V. M. Abazov et al. [D0 Collaboration].

hep-ex/0410062

Phys. Rev. Lett. 94, 091802 (2005)

1374. "Measurement of the $\Lambda_{b}^{0}$ lifetime in the decay $\Lambda_{b}^{0} \rightarrow J / \psi \Lambda^{0}$ with the D $\emptyset$ detector" V. M. Abazov et al. [D0 Collaboration].

hep-ex/0410054

Phys. Rev. Lett. 94, 102001 (2005)

1375. "Measurement of the ratio of $B^{+}$and $B^{0}$ meson lifetimes"

V. M. Abazov et al. [D0 Collaboration].

hep-ex/0410052

Phys. Rev. Lett. 94, 182001 (2005)

1376. "A search for the flavor-changing neutral current decay $B_{s}^{0} \rightarrow \mu^{+} \mu^{-}$in $p \bar{p}$ collisions at $\sqrt{s}=1.96 \mathrm{TeV}$ with the $\mathrm{D} \emptyset$ detector"

V. M. Abazov et al. [D0 Collaboration].

hep-ex/0410039

Phys. Rev. Lett. 94, 071802 (2005)

1377. "Measurement of the $B_{s}^{0}$ lifetime in the exclusive decay channel $B_{s}^{0} \rightarrow J / \psi \phi$ "

V. M. Abazov et al. [D0 Collaboration].

hep-ex/0409043

Phys. Rev. Lett. 94, 042001 (2005)

1378. "Measurement of dijet azimuthal decorrelations at central rapidities in $p \bar{p}$ collisions at $\sqrt{s}=1.96 \mathrm{TeV}^{\prime \prime}$

V. M. Abazov et al. [D0 Collaboration].

hep-ex/0409040

Phys. Rev. Lett. 94, 221801 (2005)

1379. "Search for supersymmetry with gauge-mediated breaking in diphoton events at D0"

V. M. Abazov et al. [D0 Collaboration]. 
hep-ex/0408146

Phys. Rev. Lett. 94, 041801 (2005)

1380. "Measurement of direct photon production at Tevatron fixed target energies" L. Apanasevich et al. [Fermilab E706 Collaboration].

hep-ex/0407011

Phys. Rev. D 70, 092009 (2004)

1381. "New measurement of the top quark mass in lepton + jets $t \bar{t}$ events at DØ" V. M. Abazov et al. [D0 Collaboration].

hep-ex/0407005

1382. "A precision measurement of the mass of the top quark"

V. M. Abazov et al. [D0 Collaboration].

hep-ex/0406031

Nature 429, 638 (2004)

1383. "Observation and properties of the $X(3872)$ decaying to $J / \psi \pi^{+} \pi^{-}$in $p \bar{p}$ collisions at $\sqrt{s}=1.96 \mathrm{TeV}^{\prime}$

V. M. Abazov et al. [D0 Collaboration].

hep-ex/0405004

Phys. Rev. Lett. 93, 162002 (2004)

1384. "Helicity of the $W$ boson in lepton + jets $t \bar{t}$ events"

V. M. Abazov et al. [D0 Collaboration].

hep-ex/0404040

Phys. Lett. B 617, 1 (2005)

1385. "Search for doubly-charged Higgs boson pair production in the decay to $\mu^{+} \mu^{+} \mu^{-} \mu^{-}$ in $p \bar{p}$ collisions at $\sqrt{s}=1.96 \mathbf{T e V}$ "

V. M. Abazov et al. [D0 Collaboration].

hep-ex/0404015

Phys. Rev. Lett. 93, 141801 (2004)

1386. "Search for pair production of light scalar top quarks in $p \bar{p}$ collisions at $\sqrt{s}=1.8$ TeV"

V. M. Abazov et al. [D0 Collaboration].

hep-ex/0404028

Phys. Rev. Lett. 93, 011801 (2004)

1387. "Upper limit on the decay Sigma(1385)- — i Sigma- gamma, and cross-section for gamma Sigma- - i Lambda pi-"

V. V. Molchanov et al. [SELEX Collaboration].

hep-ex/0402026

Phys. Lett. B 590, 161 (2004)

1388. "Search for 3- and 4-body decays of the scalar top quark in $p \bar{p}$ collisions at $\sqrt{s}=1.8$ TeV" 
V. M. Abazov et al. [D0 Collaboration].

Phys. Lett. B 581, 147 (2004).

1389. "Search for new particles in the two jet decay channel with the Do detector" V. M. Abazov et al. [D0 Collaboration].

hep-ex/0308033

Phys. Rev. D 69, 111101 (2004)

1390. "Observation of diffractively produced $W$ and $Z$ bosons in $\bar{p} p$ collisions at $\sqrt{s}=$ 1800 GeV"

V. M. Abazov et al. [D0 Collaboration].

hep-ex/0308032

Phys. Lett. B 574, 169 (2003)

1391. "Production of pi0 and eta mesons at large transverse momenta in pi- $\mathbf{p}$ and piBe interactions at $515-\mathrm{GeV} / \mathrm{c} "$

L. Apanasevich et al. [Fermilab E706 Collaboration].

hep-ex/0308022

Phys. Rev. D 69, 032003 (2004)

1392. "Search for narrow $t \bar{t}$ resonances in $p \bar{p}$ collisions at $\sqrt{s}=1.8 \mathrm{TeV}$ "

V. M. Abazov et al. [D0 Collaboration].

hep-ex/0307079

Phys. Rev. Lett. 92, 221801 (2004)

1393. "Search for large extra dimensions in the monojet + missing $E_{T}$ channel at DØ" V. M. Abazov et al. [D0 Collaboration].

hep-ex/0302014

Phys. Rev. Lett. 90, 251802 (2003)

1394. "Do Run IIB upgrade technical design report"

V. M. Abazov et al. [D0 Collaboration].

1395. "Investigating QCD at fixed-target energies"

P. F. Slattery.

Comments Nucl. Part. Phys. 2, A377 (2002).

1396. "Search for the production of single sleptons through $R$-parity violation in $p \bar{p}$ collisions at $\sqrt{s}=1.8 \mathrm{TeV}^{\prime \prime}$

V. M. Abazov et al. [D0 Collaboration].

hep-ex/0207100

Phys. Rev. Lett. 89, 261801 (2002)

1397. "Multiple jet production at low transverse energies in $p \bar{p}$ collisions at $\sqrt{s}=1.8$ TeV"

V. M. Abazov et al. [D0 Collaboration].

hep-ex/0207046

Phys. Rev. D 67, 052001 (2003) 
1398. " $t \bar{t}$ production cross-section in $p \bar{p}$ collisions at $\sqrt{s}=\mathbf{1 . 8 - T e V "}$

V. M. Abazov et al. [D0 Collaboration].

hep-ex/0205019

Phys. Rev. D 67, 012004 (2003)

1399. "Search for mSUGRA in single electron events with jets and large missing transverse energy in $p \bar{p}$ collisions at $\sqrt{s}=1.8 \mathrm{TeV}^{\prime}$

V. M. Abazov et al. [D0 Collaboration].

hep-ex/0205002

Phys. Rev. D 66, 112001 (2002)

1400. "Production of pi0 and eta mesons at large transverse momenta in pp and p Be interactions at $530-\mathrm{GeV} / \mathrm{c}$ and $800-\mathrm{GeV} / \mathrm{c}$ "

L. Apanasevich et al. [Fermilab E706 Collaboration].

hep-ex/0204031

Phys. Rev. D 68, 052001 (2003)

1401. "Improved $W$ boson mass measurement with the D $\emptyset$ detector"

V. M. Abazov et al. [D0 Collaboration].

hep-ex/0204014

Phys. Rev. D 66, 012001 (2002)

1402. "A direct measurement of $W$ boson decay width"

V. M. Abazov et al. [D0 Collaboration].

hep-ex/0204009

Phys. Rev. D 66, 032008 (2002)

1403. "Search for $R$-parity violating supersymmetry in dimuon and four-jets channel" V. M. Abazov et al. [D0 Collaboration].

hep-ex/0111053

Phys. Rev. Lett. 89, 171801 (2002)

1404. "Search for leptoquark pairs decaying to $\nu \nu+$ jets in $p \bar{p}$ collisions at $\sqrt{s}=1.8 \mathbf{T e V}^{\prime}$ V. M. Abazov et al. [D0 Collaboration].

hep-ex/0111047

Phys. Rev. Lett. 88, 191801 (2002)

1405. "The inclusive jet cross-section in $p \bar{p}$ collisions at $\sqrt{s}=1.8 \mathrm{TeV}$ using the $k_{T}$ algorithm"

V. M. Abazov et al. [D0 Collaboration].

hep-ex/0109041

Phys. Lett. B 525, 211 (2002)

1406. "Radiative decay width of the a(2)(1320)- meson"

V. V. Molchanov et al. [SELEX Collaboration].

hep-ex/0109016

Phys. Lett. B 521, 171 (2001) 
1407. "Subjet multiplicity of gluon and quark jets reconstructed with the $k_{T}$ algorithm in $p \bar{p}$ collisions"

V. M. Abazov et al. [D0 Collaboration].

hep-ex/0108054

Phys. Rev. D 65, 052008 (2002)

1408. "A search for the scalar top quark in $p \bar{p}$ collisions at $\sqrt{s}=1.8$ TeV"

V. M. Abazov et al. [D0 Collaboration].

hep-ex/0108018

Phys. Rev. Lett. 88, 171802 (2002)

1409. "Measurement of the ratio of differential cross sections for $W$ and $Z$ boson production as a function of transverse momentum in $p \bar{p}$ collisions at $\sqrt{s}=1.8 \mathrm{TeV}$ " V. M. Abazov et al. [D0 Collaboration].

hep-ex/0107012

Phys. Lett. B 517, 299 (2001)

1410. "A Study of multiple jet production at transverse energy near 20-GeV" V. M. Abazov et al. [D0 Collaboration].

hep-ex/0106072

1411. "Search for leptoquark pairs decaying to $\nu \nu+$ jets in $p \bar{p}$ collisions at $\sqrt{s}=1.8 \mathrm{TeV}$ " V. M. Abazov et al. [D0 Collaboration].

hep-ex/0106065

1412. "Search for single top quark production at D0 using neural networks"

V. M. Abazov et al. [D0 Collaboration].

hep-ex/0106059

Phys. Lett. B 517, 282 (2001)

1413. "Search for new physics using QUAERO: A General interface to Do event data" V. M. Abazov et al. [D0 Collaboration].

hep-ex/0106039

Phys. Rev. Lett. 87, 231801 (2001)

1414. "Measurement of the ratio of the differential cross sections for $W$ and $Z$ boson production as a function of transverse momentum in $p \bar{p}$ collisions at $\sqrt{s}=1.8$ TeV"

V. M. Abazov et al. [D0 Collaboration].

hep-ex/0106027

1415. "The inclusive jet cross section in $p \bar{p}$ collisions at $\sqrt{s}=1.8 \mathrm{TeV}$ using the $\mathbf{k}(\mathbf{T})$ algorithm"

V. M. Abazov et al. [D0 Collaboration].

hep-ex/0106032

1416. "The ratio of the isolated photon cross sections at $\sqrt{s}=630 \mathrm{GeV}$ and $1800 \mathrm{GeV}$ " V. M. Abazov et al. [D0 Collaboration]. 
hep-ex/0106026

Phys. Rev. Lett. 87, 251805 (2001)

1417. "Improved DØ $W$ boson mass determination"

V. M. Abazov et al. [D0 Collaboration].

hep-ex/0106018

1418. "Search for first generation scalar and vector leptoquarks"

V. M. Abazov et al. [D0 Collaboration].

hep-ex/0105072

Phys. Rev. D 64, 092004 (2001)

1419. "Search for heavy particles decaying into electron positron pairs in $p \bar{p}$ collisions" V. M. Abazov et al. [D0 Collaboration].

hep-ex/0102048

Phys. Rev. Lett. 87, 061802 (2001)

1420. "Direct search for charged Higgs bosons in decays of top quarks"

V. M. Abazov et al. [D0 Collaboration].

hep-ex/0102039

Phys. Rev. Lett. 88, 151803 (2002)

1421. "A quasi-model-independent search for new high $p_{T}$ physics at DØ"

B. Abbott et al. [D0 Collaboration].

hep-ex/0011071

Phys. Rev. Lett. 86, 3712 (2001)

1422. "High- $p_{T}$ jets in $\bar{p} p$ collisions at $\sqrt{s}=630 \mathrm{GeV}$ and $1800 \mathrm{GeV}$ "

B. Abbott et al. [D0 Collaboration].

hep-ex/0012046

Phys. Rev. D 64, 032003 (2001)

1423. "A Quasi model independent search for new physics at large transverse momentum"

V. M. Abazov et al. [D0 Collaboration].

hep-ex/0011067

Phys. Rev. D 64, 012004 (2001)

1424. "Inclusive jet production in $p \bar{p}$ collisions"

B. Abbott et al. [D0 Collaboration].

hep-ex/0011036

Phys. Rev. Lett. 86, 1707 (2001)

1425. "Differential cross section for $W$ boson production as a function of transverse momentum in $p \bar{p}$ collisions at $\sqrt{s}=1.8 \mathrm{TeV}^{\prime \prime}$

B. Abbott et al. [D0 Collaboration].

hep-ex/0010026

Phys. Lett. B 513, 292 (2001) 
1426. "Measurement of the angular distribution of electrons from $W \rightarrow e \nu$ decays observed in $p \bar{p}$ collisions at $\sqrt{s}=1.8 \mathrm{TeV}^{\prime \prime}$

B. Abbott et al. [D0 Collaboration].

hep-ex/0009034

Phys. Rev. D 63, 072001 (2001)

1427. "Ratios of multijet cross sections in $p \bar{p}$ collisions at $\sqrt{s}=1.8 \mathbf{T e V}$ "

B. Abbott et al. [D0 Collaboration].

hep-ex/0009012

Phys. Rev. Lett. 86, 1955 (2001)

1428. "The ratio of jet cross sections at $\sqrt{s}=630 \mathrm{GeV}$ and $1800 \mathrm{GeV}$ "

B. Abbott et al. [D0 Collaboration].

hep-ex/0008072

Phys. Rev. Lett. 86, 2523 (2001)

1429. "Search for large extra dimensions in dielectron and diphoton production"

B. Abbott et al. [D0 Collaboration].

hep-ex/0008065

Phys. Rev. Lett. 86, 1156 (2001)

1430. "Search for electroweak production of single top quarks in $p \bar{p}$ collisions"

B. Abbott et al. [D0 Collaboration].

hep-ex/0008024

Phys. Rev. D 63, 031101 (2000)

1431. "Cross section for $\mathbf{b}$ jet production in $\bar{p} p$ collisions at $\sqrt{s}=1.8$ TeV"

B. Abbott et al. [D0 Collaboration].

hep-ex/0008021

Phys. Rev. Lett. 85, 5068 (2000)

1432. "Examination of direct photon and pion production in proton nucleon collisions" L. Apanasevich, M. Begel, C. Bromberg, T. Ferbel, G. Ginther, J. Huston, S. E. Kuhlmann and P. Slattery et al..

hep-ph/0007191

Phys. Rev. D 63, 014009 (2001)

1433. "Search for new physics in $e \mu X$ data at DØ using Sherlock: A quasi model independent search strategy for new physics"

B. Abbott et al. [D0 Collaboration].

hep-ex/0006011

Phys. Rev. D 62, 092004 (2000)

1434. "Search for $R$-parity violation in multilepton final states in $p \bar{p}$ collisions at $\sqrt{s}=$ 1.8 TeV"

B. Abbott et al. [D0 Collaboration].

hep-ex/0005034

Phys. Rev. D 62, 071701 (2000) 
1435. "Inclusive production of omega mesons at large transverse momenta in pi-Be interactions at $515-\mathrm{GeV} / \mathrm{c} "$

L. Apanasevich et al. [E706 Collaboration].

hep-ex/0004012

1436. "A search for dilepton signatures from minimal low-energy supergravity in $p \bar{p}$ collisions at $\sqrt{s}=1.8 \mathbf{T e V}^{\prime}$

B. Abbott et al. [D0 Collaboration].

Phys. Rev. D 63, 091102 (2001).

1437. "Spin correlation in $t \bar{t}$ production from $p \bar{p}$ collisions at $\sqrt{s}=1.8$ TeV"

B. Abbott et al. [D0 Collaboration].

hep-ex/0002058

Phys. Rev. Lett. 85, 256 (2000)

1438. "A measurement of the $W \rightarrow \tau \nu$ production cross section in $p \bar{p}$ collisions at $\sqrt{s}=$ 1.8 TeV"

B. Abbott et al. [D0 Collaboration].

hep-ex/9912065

Phys. Rev. Lett. 84, 5710 (2000)

1439. "Hard single diffraction in $\bar{p} p$ collisions at $\sqrt{s}=630 \mathrm{GeV}$ and $1800 \mathrm{GeV}$ "

B. Abbott et al. [D0 Collaboration].

hep-ex/9912061

Phys. Lett. B 531, 52 (2002)

1440. "Limits on anomalous $W W \gamma$ and $W W Z$ couplings from $W W / W Z \rightarrow e \nu j j$ production"

B. Abbott et al. [D0 Collaboration].

hep-ex/9912033

Phys. Rev. D 62, 052005 (2000)

1441. "Probing BFKL dynamics in the dijet cross section at large rapidity intervals in $p \bar{p}$ collisions at $\sqrt{s}=1800 \mathrm{GeV}$ and $\mathbf{6 3 0 - \mathrm { GeV } ^ { \prime }}$

B. Abbott et al. [D0 Collaboration].

hep-ex/9912032

Phys. Rev. Lett. 84, 5722 (2000)

1442. "Limits on quark compositeness from high-energy jets in $\bar{p} p$ collisions at 1.8 TeV" B. Abbott et al. [D0 Collaboration].

hep-ex/9912023

Phys. Rev. D 62, 031101 (2000)

1443. "The isolated photon cross-section in $p \bar{p}$ collisions at $\sqrt{s}=1.8 \mathrm{TeV}$ "

B. Abbott et al. [D0 Collaboration].

hep-ex/9912017

Phys. Rev. Lett. 84, 2786 (2000) 
1444. "Search for second generation leptoquark pairs in $\bar{p} p$ collisions at $\sqrt{s}=1.8 \mathrm{TeV}$ " B. Abbott et al. [D0 Collaboration].

hep-ex/9910040

Phys. Rev. Lett. 84, 2088 (2000)

1445. "Production of $J / \psi$ mesons in $p$ Be collisions at 530-GeV/c and 800-GeV/c"

A. Gribushin et al. [E672 and E706 Collaborations].

hep-ex/9910005

Phys. Rev. D 62, 012001 (2000)

1446. "A measurement of the $W$ boson mass using electrons at large rapidities"

B. Abbott et al. [D0 Collaboration].

hep-ex/9909030

Phys. Rev. Lett. 84, 222 (2000)

1447. "Differential production cross section of $Z$ bosons as a function of transverse momentum at $\sqrt{s}=1.8 \mathbf{T e V}^{\prime}$

B. Abbott et al. [D0 Collaboration].

hep-ex/9909020

Phys. Rev. Lett. 84, 2792 (2000)

1448. "A measurement of the $W$ boson mass using large rapidity electrons"

B. Abbott et al. [D0 Collaboration].

hep-ex/9908057

Phys. Rev. D 62, 092006 (2000)

1449. "Evidence of color coherence effects in $W+$ jets events from $p \bar{p}$ collisions at $\sqrt{s}=1.8$ TeV"

B. Abbott et al. [D0 Collaboration].

hep-ex/9908017

Phys. Lett. B 464, 145 (1999)

1450. "Subjet multiplicity in quark and gluon jets at D0"

B. Abbott et al. [D0 Collaboration].

hep-ex/9907059

Nucl. Phys. Proc. Suppl. 79, 494 (1999)

1451. " $k_{T}$ effects in direct photon production"

L. Apanasevich, C. Balazs, C. Bromberg, J. Huston, A. Maul, W. K. Tung, S. Kuhlmann and J. F. Owens et al..

hep-ph/9808467

Phys. Rev. D 59, 074007 (1999)

1452. "Design and performance of the Fermilab E781 (SELEX) hardware scattering trigger"

A. Atamantchuk et al. [E781 Collaboration].

Nucl. Instrum. Meth. A 425, 529 (1999). 
1453. "Evidence for parton $k_{T}$ effects in high $p_{T}$ particle production" L. Apanasevich et al. [Fermilab E706 Collaboration].

hep-ex/9711017

Phys. Rev. Lett. 81, 2642 (1998)

1454. "Calibration and performance of the E706 lead and liquid argon electromagnetic calorimeter"

L. Apanasevich et al. [E706 Collaboration].

Nucl. Instrum. Meth. A 417, 50 (1998).

1455. "Production of charm mesons at high transverse momentum in 515-GeV/c pinucleon collisions"

L. Apanasevich et al. [Fermilab E706 Collaboration].

hep-ex/9702014

Phys. Rev. D 56, 1391 (1997)

1456. "ATLAS calorimeter performance Technical Design Report"

A. Airapetian et al. [ATLAS Collaboration].

1457. "ATLAS computing technical proposal"

A. Airapetian et al. [ATLAS Collaboration].

1458. "Production of charmonium states in $\pi^{-}$Be collisions at $515-\mathrm{GeV} / \mathrm{c}$ "

V. Koreshev et al. [E672-E706 Collaboration].

Phys. Rev. Lett. 77, 4294 (1996).

1459. "Production of $\mathbf{J} /$ psi and psi (2S) mesons in pi- Be collisions at 515-GeV/c"

A. Gribushin et al. [E672 and E706 Collaboration].

Phys. Rev. D 53, 4723 (1996).

1460. "ATLAS: Technical proposal for a general-purpose p p experiment at the Large Hadron Collider at CERN"

W. W. Armstrong et al. [ATLAS Collaboration].

1461. "Bottom production in $\pi^{-}-$Be collisions at $515-\mathrm{GeV} / \mathrm{c}$ "

R. Jesik et al. [E672/E706 Collaboration].

Phys. Rev. Lett. 74, 495 (1995).

1462. "E823 (D0 upgrade): Magnetic tracking"

B. Gomez et al. [D0 Collaboration].

1463. "Structure of the recoiling system in direct photon and $\pi^{0}$ production by $\pi^{-}$and $p$ beams at $500-\mathrm{GeV} / \mathrm{c}$ "

G. Alverson et al. [E706 Collaboration].

Phys. Rev. D 49, 3106 (1994).

1464. "GEM Technical Design Report"

W. C. Lefmann et al. [GEM Collaboration]. 
1465. "Production of direct photons and neutral mesons at large transverse momenta by $\pi^{-}$and $p$ beams at $500-\mathrm{GeV} / \mathrm{c}^{\prime \prime}$

G. Alverson et al. [FERMILAB-E706 Collaboration].

Phys. Rev. D 48, 5 (1993).

1466. "Hadroproduction of $\mathrm{chi}(\mathrm{c})$ states in $530-\mathrm{GeV} / \mathrm{c}$ pi- interactions with nuclear targets"

A. Zieminski et al. [672 and E706. Collaboration].

In *Dallas 1992, Proceedings, High energy physics, vol. 1* 1062-1065

1467. "Heavy flavor production in $\pi^{-}$A collisions at $530-\mathrm{GeV} / \mathrm{c}$ "

R. Jesik et al. [E672 and E706. Collaboration].

AIP Conf. Proc. 272, 824 (1993).

1468. "Testing of components for the GEM detector at the Supercollider Laboratory: A Proposal to the Fermi National Accelerator Laboratory"

B. Barish, G. Yost, H. Kobrak, B. Willis and P. Slattery.

1469. "High Quality Muon Detection for the SSC"

W. X. Gao, J. Li, Z. P. Mao, Y. T. Xu, W. G. Yan, Y. Yang, M. H. Ye and Z. P. Zheng et al..

1470. "Direct photon results from E706"

P. F. Slattery [E-706 Collaboration].

Conf. Proc. C 910725V1, 452 (1991).

1471. "Production of pi0 mesons at high p-transverse in pi- Be and p Be collisions at $500-\mathrm{GeV} / \mathrm{c} "$

G. Alverson, W. F. Baker, G. Ballocchi, R. Benson, D. Berg, S. Blusk, C. Bromberg and D. Brown et al..

Phys. Rev. D 45, 3899 (1992).

1472. "Direct photon production at high p-transverse in pi- Be and p Be collisions at $500-\mathrm{GeV} / \mathrm{c} "$

G. Alverson, W. F. Baker, G. Ballocchi, R. Benson, D. Berg, S. Blusk, C. Bromberg and D. Brown et al..

Phys. Rev. Lett. 68, 2584 (1992).

1473. "Letter of intent, direct photon production \#834"

P. F. Slattery [E706 Collaboration].

1474. "Prompt photon experiments using polarized beams"

P. F. Slattery.

1475. "Cryogenic design and operation of a liquid argon photon / hadron calorimeter at Fermilab. FNAL E-706 experiment"

J. Urbin, W. Baker, W. DeSoi, G. Fanourakis, G. Ginther, J. Huston, A. Lanaro and C. Lirakis et al..

Adv. Cryog. Eng. 35B, 1803 (1990). 
1476. "Design and operation of large straw tube drift chamber planes"

C. Bromberg et al. [E706 Collaboration].

Nucl. Instrum. Meth. A 307, 292 (1991).

1477. "Scattering of e- and production of e+ in strong electromagnetic fields" D. Burke, R. C. Field, G. Horton-Smith, T. Kotseroglou, J. E. Spencer, D. Walz, S. C. Berridge and W. M. Bugg et al..

1478. "The Sagnac effect and the Tevatron"

A. Melissinos.

arXiv:1101.4008 [physics.acc-ph]

1479. "The response of laser interferometers to a gravitational wave"

A. Melissinos and A. Das.

arXiv:1002.0809 [gr-qc]

1480. "Breakdown of the vacuum by a strong gravitational field"

A. C. Melissinos.

Int. J. Mod. Phys. E 17, 891 (2008).

1481. "Search for Cosmic Axions using an Optical Interferometer"

A. C. Melissinos.

arXiv:0807.1092 [hep-ph]

Phys. Rev. Lett. 102, 202001 (2009)

1482. "Comment on Experimental Observation of Optical Rotation Generated in Vacuum by a Magnetic Field"

A. C. Melissinos.

hep-ph/0702135

1483. "Search for higher dimensions through their gravitational effects in high energy collisions"

A. C. Melissinos.

hep-ph/0604151

1484. "Performance of the upgraded laser system for the Fermilab-NIU photoinjector" J. -L. Li, R. Tikhoplav and A. C. Melissinos.

Nucl. Instrum. Meth. A 564, 57 (2006).

1485. "Simulation of the laser acceleration experiment at the Fermilab/NICADD photoinjector laboratory"

P. Piot, R. Tikhoplav and A. C. Melissinos.

Conf. Proc. C 0505161, 2503 (2005).

1486. "Production of transverse controllable laser density distribution in Fermilab/NICADD photoinjector"

J. -L. Li, R. Tikoplav, P. Piot and A. Melissinos.

Conf. Proc. C 0505161, 2783 (2005). 
1487. "Measurement of a stochastic gravitational wave background with a single laser interferometer"

A. C. Melissinos and W. E. Butler.

gr-qc/0501089

1488. "The Energetics of particle acceleration using high intensity lasers"

A. C. Melissinos.

physics/0410273

1489. "Experimental investigation of the longitudinal beam dynamics in a photoinjector using a two-macroparticle bunch"

R. Tikhoplav et al. [Fermilab NICADD Photoinjector Laboratory Collaboration].

1490. "Laser acceleration of electrons"

R. Tikhoplav, A. Melissinos, N. Barov and J. Santucci.

1491. “A 'Running' gravitational constant?"

A. C. Melissinos.

hep-ph/0207282

1492. "Measuring the phase velocity of light in a magnetic field with the PVLAS detector"

A. C. Melissinos.

hep-ph/0205169

1493. "Spontaneous breakdown of the vacuum by intense lasers"

A. C. Melissinos.

1494. "Vacuum energy and the cosmological constant"

A. C. Melissinos.

hep-ph/0112266

1495. "Electro-optic measurement of the wake fields of a relativistic electron beam" M. J. Fitch, A. C. Melissinos, P. L. Colestock, J. P. Carneiro, H. T. Edwards and W. H. Hartung.

Phys. Rev. Lett. 87, 034801 (2001).

1496. "Neutrino scattering in a magnetic field"

K. S. McFarland, A. C. Melissinos, N. V. Mikheev and W. W. Repko.

hep-ph/0106196

1497. "Electrooptic sampling of transient fields from the passage of high charge electron bunches"

M. J. Fitch, A. C. Melissinos, P. L. Colestock, J. P. Carneiro, H. T. Edwards and W. H. Hartung.

physics/0008207 [physics.acc-ph], physics/0008207 [physics.acc-ph]

eConf C 000821, MOC05 (2000) 
1498. "Compression of high charge electron bunches"

M. J. Fitch, A. C. Melissinos, N. Barov, J. P. Carneiro, H. T. Edwards and W. H. Hartung. physics/0008206 [physics.acc-ph], physics/0008206 [physics.acc-ph]

eConf C 000821, MOB16 (2000)

1499. "A0 Photo-Injector: Experiments with Gun A June 97 to December 97"

J. P. Carneiro, R. A. Carrigan, M. S. Champion, A. Cianchi, E. R. Colby, P. L. Colestock, H. T. Edwards and J. D. Fuerst et al..

1500. "Possible and impossible experiments"

A. C. Melissinos.

1501. "Electrooptic measurement of the wake fields of 16-MeV electron bunches" M. J. Fitch, N. Barov, J. P. Carneiro, P. L. Colestock, H. T. Edwards, K. P. Koepke, A. C. Melissinos and W. H. Hartung.

1502. "First results of the Fermilab high brightness RF photoinjector"

J. P. Carneiro, R. A. Carrigan, M. S. Champion, P. L. Colestock, H. T. Edwards, J. D. Fuerst, W. H. Hartung and K. P. Koepke et al..

1503. "Picosecond electron bunch length measurement by electrooptic detection of the wake field"

M. J. Fitch, A. C. Melissinos and P. L. Colestock.

1504. "Studies of nonlinear QED in collisions of 46.6-GeV electrons with intense laser pulses"

C. Bamber, S. J. Boege, T. Koffas, T. Kotseroglou, A. C. Melissinos, D. D. Meyerhofer, D. A. Reis and W. Ragg et al..

Phys. Rev. D 60, 092004 (1999).

1505. "Locking of an NPRO / YAG oscillator to a finesse 1000 Fabry-Perot cavity" T. Koffas, T. Blalock, S. Campbell and A. C. Melissinos.

1506. "Quantum aspects, experimental results, and beam physics implications of E144 "

D. L. Burke et al. [E-144 Collaboration].

1507. "Beam transport, acceleration and compression studies in the Fermilab high brightness photoinjector"

J. -P. Carneiro, R. A. Carrigan, M. S. Champion, A. Cianchi, E. R. Colby, P. L. Colestock, H. T. Edwards and J. D. Fuerst et al..

1508. "Observation of stimulated raman scattering in an optical fiber at the Fermilab A0 photoinjector"

A. R. Fry, B. Taylor, M. J. Fitch and A. C. Melissinos.

1509. "A Test experiment at the Fermilab Booster to study the feasibility of fast antihydrogen spectroscopy"

G. Blanford, M. Mandelkern, J. Schultz, G. Zioulas, D. Christian, K. Gollwitzer, A. Hahn and F. Nezrick et al.. 
1510. "Alternatives to axion production and detection"

A. C. Melissinos.

hep-ph/9805506

Nucl. Phys. Proc. Suppl. 72, 195 (1999)

1511. "The Spontaneous breakdown of the vacuum"

A. C. Melissinos.

hep-ph/9805507

1512. "Laser System for the TTF Photoinjector at Fermilab"

A. R. Fry, M. J. Fitch, A. C. Melissinos, N. P. Bigelow, B. D. Taylor and F. A. Nezrick.

Conf. Proc. C 970512, 2867 (1997).

1513. "Production of MeV gamma-rays at the TTF"

A. C. Melissinos and J. S. T. Ng.

1514. "Positron production in multi - photon light by light scattering"

D. L. Burke, R. C. Field, G. Horton-Smith, T. Kotseroglou, J. E. Spencer, D. Walz, S. C. Berridge and W. M. Bugg et al..

Phys. Rev. Lett. 79, 1626 (1997).

1515. "Compton scattering x-ray experiments at the Fermilab electron source facility"

A. Melissinos, N. P. Bigelow, M. J. Fitch, A. R. Fry, P. L. Colestock, H. T. Edwards and F. A. Nezrick.

1516. "An update of results from an experiment to search for galactic axions"

S. De Panfilis, A. Melissinos, B. Moskowitz, J. Rogers, Y. Semertzidis, W. Wuensch, H. Halama and A. Prodell et al..

In *Annecy 1988, Low temperature detectors for neutrinos and dark matter* 67-79

1517. "Positron production in multi - photon light by light scattering"

C. Bamber, S. C. Berridge, S. J. Boege, W. M. Bugg, C. Bula, D. L. Burke, R. C. Field and G. Horton-Smith et al..

AIP Conf. Proc. 396, 165 (1997).

1518. "Particle astrophysics"

A. C. Melissinos.

1519. "Observation of nonlinear effects in Compton scattering"

C. Bula et al. [E144 Collaboration].

Phys. Rev. Lett. 76, 3116 (1996).

1520. "Picosecond timing of terawatt laser pulses with the SLAC 46-GeV electron beam"

T. Kotseroglou et al. [E144 Collaboration].

Nucl. Instrum. Meth. A 383, 309 (1996).

1521. "Laser electron interactions"

A. Melissinos.

SLAC Beam Line 25N3, 31 (1995), [SLAC Beam Line 25, 31 (1995)]. 
1522. "Preliminary observation of nonlinear effects in Compton scattering" C. Bula et al. [E144 Collaboration].

1523. "Electron emission from metallic surfaces by picosecond laser pulses" A. R. Fry and A. C. Melissinos.

1524. "Search for nearly massless, weakly coupled particles by optical techniques" R. Cameron, G. Cantatore, A. C. Melissinos, G. Ruoso, Y. Semertzidis, H. J. Halama, D. M. Lazarus and A. G. Prodell et al.. Phys. Rev. D 47, 3707 (1993).

1525. "Electron acceleration using laser driven photoconductive switching" C. Bamber, W. Donaldson, E. Lincke and A. C. Melissinos. Nucl. Instrum. Meth. A 327, 227 (1993).

1526. "Energy measurement by resonant depolarization" A. C. Melissinos. In $*$ Sudarshan, E.C.G. (ed.): A gift of prophecy* $324-338$

1527. "Nicholas C. Christofilos: His contributions to physics" A. C. Melissinos. In *Rhodos 1993, Proceedings, Advanced accelerator physics* 1067-1081.

1528. "A pulsed-power electron accelerator using laser driven photoconductive switches" C. Bamber, W. R. Donaldson, E. Lincke and A. C. Melissinos. AIP Conf. Proc. 279, 802 (1993).

1529. "A novel high brilliance electron source" W. R. Donaldson and A. C. Melissinos. AIP Conf. Proc. 279, 791 (1993).

1530. "Coherent production / detection of light scalars / pseudoscalars and QED vacuum polarization"

Y. K. Semertzidis, R. Cameron, G. Cantatore, A. C. Melissinos, G. Ruoso, H. J. Halama, D. M. Lazarus and A. G. Prodell et al..

In *Dallas 1992, Proceedings, High energy physics, vol. 2* 1266-1270

1531. "A Search for solar axions"

D. M. Lazarus, G. C. Smith, R. Cameron, A. C. Melissinos, G. Ruoso, Y. K. Semertzidis and F. A. Nezrick.

Phys. Rev. Lett. 69, 2333 (1992).

1532. "Limits on light scalar and pseudoscalar particles from a photon regeneration experiment"

G. Ruoso, R. Cameron, G. Cantatore, A. C. Melissinos, Y. Semertzidis, H. J. Halama, D. M. Lazarus and A. G. Prodell et al..

Zeit. Phys. C 56, 505 (1992). 
1533. "A search for the coherent production of axions in the milli eV range"

R. Cameron, A. C. Melissinos, Y. Semertzidis, G. Cantatore, C. Rizzo, P. Ruoso, E. Zavattini and H. Halama et al..

In *Vancouver 1991, Particles and fields '91, vol. 2* 1002-1004

1534. "The laser switched linac and development of a high brilliance electron source" A. C. Melissinos, C. Bamber, T. Blalock, A. Fry and T. Wilson.

1535. "Study of QED at critical field strength in intense laser - high-energy electron collisions"

K. T. McDonald, C. Bamber, A. C. Melissinos, D. Meyerhoffer, P. Chen, J. E. Spencer and R. B. Palmer.

In *Los Angeles 1991, Proceedings, Beam-beam and beam-radiation interactions* 127-156.

1536. "Proposal for a study of QED at critical field strength in intense laser highenergy electron collisions at the Stanford Linear Accelerator"

J. G. Heinrich, C. Lu, K. T. McDonald, C. Bamber, A. C. Melissinos, D. Meyerhofer, Y. Semertzidis and P. Chen et al..

1537. "First measurement of the magnetic birefringence of helium gas"

R. Cameron, G. Cantatore, A. C. Melissinos, Y. Semertzidis, H. Halama, D. Lazarus, A. Prodell and F. Nezrick et al..

Phys. Lett. A 157, 125 (1991).

1538. "Acceleration of picosecond electron bunches in a radial transmission line" C. Bamber, W. Donaldson, L. Kingsley, E. Lincke and A. C. Melissinos.

Conf. Proc. C 900612, 609 (1990).

1539. "Cosmic axions. Proceedings, Workshop, Upton, USA, April 13-14, 1989"

C. Jones, (ed.) and A. Melissinos, (ed.).

Singapore, Singapore: World Scientific (1990) 319 p

1540. "Limits on the lifetime of high-energy storage rings due to thermal photon scattering"

T. Kotseroglou and A. C. Melissinos.

1541. "Scattering of high-energy electrons off thermal photons"

B. Dehning, A. C. Melissinos, F. Perrone, C. Rizzo and G. von Holtey.

Phys. Lett. B 249, 145 (1990).

1542. "Measurement Of The Magnetic Birefringence Of Neon Gas"

R. Cameron, G. Cantatore, A. C. Melissinos, J. Rogers, Y. Semertzidis, H. Halama, A. Prodell and F. A. Nezrick et al..

J. Opt. Soc. Am. B 8, 520 (1991).

1543. "Measurement Of The Magnetic Birefringence Of Neon Gas"

R. Cameron, G. Cantatore, A. C. Melissinos, J. Rogers, Y. Semertzidis, H. Halama, A. Prodell and F. A. Nezrick et al.. 
1544. "Limits On The Production Of Light Scalar And Pseudoscalar Particles" Y. Semertzidis, R. Cameron, G. Cantatore, A. C. Melissinos, J. Rogers, H. Halama, A. Prodell and F. Nezrick et al..

Phys. Rev. Lett. 64, 2988 (1990).

1545. "Scattering Of The Lep Beam From The 300-degree K Thermal Radiation: A Possible Measurement"

A. C. Melissinos and G. von Holtey.

1546. "Laser system for a high duty cycle photoinjector"

A. R. Fry, M. J. Fitch, A. C. Melissinos and B. D. Taylor.

Nucl. Instrum. Meth. A 430, 180 (1999).

1547. "Report of the working group on gravitational wave detection"

G. Gratta, K. -J. Kim, A. Melissinos and T. Tauchi.

In *Los Angeles 1991, Proceedings, Beam-beam and beam-radiation interactions* $70-83$ and

Rochester Univ. - UR 1218 (91,rec.Oct.) 14 p

1548. "Top-mass measurements from D0"

T. Ferbel [D0 Collaboration].

PoS EPS -HEP2009, 364 (2009).

1549. "R \& D for Future Zeplin"

R. Bisset, M. J. Carson, H. Chagani, D. B. Cline, E. J. Daw, T. Ferbel, J. Gao and Y. S. Gao et al..

arXiv:0705.2117 [astro-ph]

Nucl. Phys. Proc. Suppl. 173, 164 (2007)

1550. "Introduction to nuclear and particle physics: Solutions manual for second edition"

C. Bromberg, A. Das and T. Ferbel.

Hackensack, USA: World Scientific (2006) 172 p

1551. "Lifetimes and lifetime ratios of b hadrons obtained at D0"

T. Ferbel [D0 Collaboration].

PoS HEP 2005, 226 (2006).

1552. "Recent advancements in the development of radiation hard semiconductor detectors for S-LHC"

E. Fretwurst et al. [CERN RD50 Collaboration].

Nucl. Instrum. Meth. A 552, 7 (2005).

1553. "Radiation-hard semiconductor detectors for SuperLHC"

M. Bruzzi, J. Adey, A. Al-Ajili, P. Alexandrov, G. Alfieri, P. P. Allport, A. Andreazza and M. Artuso et al..

Nucl. Instrum. Meth. A 541, 189 (2005).

1554. "ZEPLIN IV: A future large-scale liquid xenon dark matter detector"

M. Atac, Y. Chen, D. B. Cline, T. Ferbel, J. Gao, U. Schroeder, F. Sergiampietri and 
W. Skulski et al..

New Astron. Rev. 49, 283 (2005).

1555. "Status of the ZEPLIN II experiment"

G. J. Alner, M. Atac, A. Bewick, C. Bungau, B. Camanzi, M. J. Carson, C. Ghag and Y. Chen et al..

New Astron. Rev. 49, 259 (2005).

1556. "Nuclear effects in high-p(T) production of direct photons and neutral mesons" L. Apanasevich et al. [Fermilab E706 Collaboration].

hep-ex/0506003

Phys. Rev. D 72, 032003 (2005)

1557. "Upper limit on the decay Sigma(1385)- — i Sigma- gamma, and cross-section for gamma Sigma- - i Lambda pi-"

V. V. Molchanov et al. [SELEX Collaboration].

hep-ex/0402026

Phys. Lett. B 590, 161 (2004)

1558. "Search for 'large' extra dimensions at the Tevatron"

T. Ferbel.

hep-ex/0103009

1559. "Probing luminous and dark matter. Proceedings, Symposium in Honor of Adrian Melissinos, Rochester, USA, September 24-25, 1999"

A. K. Das, (ed.) and T. Ferbel, (ed.).

Singapore, Singapore: World Scientific (2000) 290 p

1560. "Inclusive direct photon and pion production in proton nucleon collisions"

T. Ferbel.

hep-ph/0009175

1561. "An update on the properties of the top quark"

T. Ferbel.

1562. "Current status of GEM: The best collider experiment at the SSC"

T. Ferbel [GEM Collaboration].

Nucl. Phys. Proc. Suppl. 32, 3 (1998).

1563. "Radiative width of the a(2) meson"

J. Russ et al. [SELEX Collaboration].

hep-ex/9901014

In *Vancouver 1998, High energy physics, vol. 2* 1296-1299

1564. "First charm hadroproduction results from SELEX"

J. Russ et al. [SELEX Collaboration].

hep-ex/9812031

In *Vancouver 1998, High energy physics, vol. 2* 1259-1262 
1565. "Hyperon physics results from SELEX"

I. M. Gough Eschrich et al. [SELEX Collaboration].

hep-ex/9812019

In *Batavia 1998, Heavy quarks at fixed target* 303-313

1566. "Techniques and concepts of high-energy physics IX. Proceedings, 9th NATO Advanced Study Institute, St. Croix, USA, July 11-22, 1996"

T. Ferbel, (ed.).

NATO Adv. Study Inst. Ser. C. Math. Phys. Sci. 356, 1 (1997).

1567. "High pressure monitored drift tube chambers for the ATLAS detector at the Large Hadron Collider"

U. Bratzler, T. Ferbel, A. Gabutti, H. Kroha, T. Lagouri, A. Manz and M. Treichel.

In $*$ Leon 1997 , Instrumentation in elementary particle physics* $371-374$

1568. "Round table discussion on direct photons"

P. Aurenche and T. Ferbel.

NATO Adv. Study Inst. Ser. B Phys. 197, 205 (1987).

1569. "Direct gamma and meson production in a QCD inspired phenomenological framework"

T. Ferbel [E706 Collaboration].

1570. "Kinematic fitting for the mass of the top quark"

T. Ferbel [D0 Collaboration].

1571. "Performance of a large scale scintillating fiber tracker using VLPC readout"

D. Adams, M. Adams, B. Baumbaugh, I. Bertram, A. Bross, D. Casey, S. Chang and M. Chung et al..

IEEE Trans. Nucl. Sci. 42, 401 (1995).

1572. "Techniques and concepts of high-energy physics. Proceedings, 8th NATO Advanced Study Institute, St. Croix, USA, June 16-27, 1994"

T. Ferbel, (ed.).

NATO Adv. Study Inst. Ser. B Phys. 351, 1 (1995).

1573. "Techniques and concepts of high-energy physics. Proceedings, 7th NATO Advanced Study Institute, St. Croix, USA, July 15-26, 1992"

T. Ferbel, (ed.).

NATO Adv. Study Inst. Ser. B Phys. 322, 1 (1994).

1574. "Introduction to nuclear and particle physics"

A. K. Das and T. Ferbel.

New York, USA: Wiley (1994) 327 p

1575. "Hybrid mesons and other physics issues in Coulomb excitation at high energies" T. Ferbel.

In *Sudarshan, E.C.G. (ed.): A gift of prophecy* 149-155 
1576. "Fiber tracking"

D. Adams, M. Adams, B. Baumbaugh, I. Bertram, A. Bross, D. Casey, S. Chang and M. Chung et al..

In *Batavia 1994, The future of high-sensitivity charm experiments* 173-192

1577. "D0 results on QCD and on the search for top"

T. Ferbel [D0 Collaboration].

1578. "Advanced technology and particle physics. Proceedings, 3rd International Conference, Como, Italy, June 22-26, 1992"

E. Borchi, (ed.), T. Ferbel, (ed.), D. Nygren, (ed.), A. Penzo, (ed.) and P. G. Rancoita, (ed.). Amsterdam, Netherlands: North-Holland (1993) 566 p. (Nucl. Phys. B, Proc. Suppl. 32 (1993))

1579. "Nuclear effects in inclusive production at large $\mathbf{p}(\mathrm{T})$ "

T. Ferbel.

1580. "High Quality Muon Detection for the SSC"

W. X. Gao, J. Li, Z. P. Mao, Y. T. Xu, W. G. Yan, Y. Yang, M. H. Ye and Z. P. Zheng et al..

1581. "Experimental Techniques In High-energy Physics"

T. Ferbel.

MENLO PARK, USA: ADDISON-WESLEY (1987) 678 P. (FRONTIERS IN PHYSICS, 64)

1582. "Direct photon production with pi- and proton beams"

T. Ferbel.

In *Warsaw 1991, Proceedings, Puzzles on the electroweak scale* $563-566$

1583. "Techniques and concepts of high-energy physics. Proceedings, 6th NATO Advanced Study Institute, St. Croix, USA, June 14-25, 1990"

T. Ferbel, (ed.).

NATO Adv. Study Inst. Ser. B Phys. 275, 1 (1991).

1584. "GEM Letter of Intent"

R. Steiner et al. [GEM Collaboration].

1585. "An Expression of Interest to Construct a Major SSC Detector"

R. V. Steiner et al. [GEM Collaboration].

1586. "Advanced technology and particle physics. Proceedings, International Conference, Como, Italy, June 11-15, 1990"

E. Borchi, (ed.), A. Penzo, (ed.), P. G. Rancoita, (ed.), T. Ferbel, (ed.) and D. Nygren, (ed.). Amsterdam, Netherlands: North-Holland (1991) 467 p. (Nucl. Phys. B, Proc. Suppl. 23A (1991))

1587. "Monte Carlo studies of response of a liquid argon calorimeter with lead absorber"

C. Pun and T. Ferbel.

Nucl. Instrum. Meth. A 302, 285 (1991). 
1588. "Search for Primakoff production of hybrid mesons"

V. Chaloupka, T. Zhao, T. Ferbel, F. Lobkowicz and M. Zielinski.

1589. "A Measurement of e / pi for a fast lead liquid argon calorimeter"

D. Makowiecki, G. S. Abrams, K. Amako, A. R. Baden, T. Bowen, T. Burnett, V. Cook and L. Cremaldi et al..

In *Fort Worth 1990, Proceedings, Detector research and development for the Superconducting Super Collider* 354-356

1590. "Comments on meson spectroscopy"

T. Ferbel.

Conf. Proc. C 900802V1 (19??).

1591. "Techniques and concepts of high-energy physics. Proceedings, 5th NATO Advanced Study Institute, St. Croix, USA, July 14-25, 1988"

T. Ferbel, (ed.).

NATO Adv. Study Inst. Ser. B Phys. 204, 1 (1990).

1592. "Letter of Intent to Write a Proposal for an Experiment to be Performed at the SSC by EMPACT/TEXAS"

R. Steiner et al. [EMPACT Collaboration].

1593. "EMPACT: Electrons Muons Partons as Air Core Toroids: An Expression of Interest for an Experiment to be Performed at the SSC"

R. Steiner et al. [EMPACT Collaboration].

1594. "Improving Energy Resolution Of Calorimeters Using A Covariance Matrix Approach"

D. Kewley, G. C. Blazey, J. Chiu and T. Ferbel.

Nucl. Instrum. Meth. A 290, 346 (1990).

1595. "The MINER $\nu$ A Data Acquisition System and Infrastructure"

G. N. Perdue et al. [MINERvA Collaboration].

arXiv:1209.1120 [physics.ins-det]

1596. "Differences in Quasi-Elastic Cross-Sections of Muon and Electron Neutrinos"

M. Day and K. S. McFarland.

arXiv:1206.6745 [hep-ph]

1597. "First Muon-Neutrino Disappearance Study with an Off-Axis Beam"

K. Abe et al. [T2K Collaboration].

arXiv:1201.1386 [hep-ex]

Phys. Rev. D 85, 031103 (2012)

1598. "Arachne - A web-based event viewer for MINERvA"

N. Tagg et al. [MINERvA Collaboration].

arXiv:1111.5315 [hep-ex]

Nucl. Instrum. Meth. 676, 44 (2012) 
1599. "Measurements of the T2K neutrino beam properties using the INGRID on-axis near detector"

K. Abe, N. Abgrall, Y. Ajima, H. Aihara, J. B. Albert, C. Andreopoulos, B. Andrieu and M. D. Anerella et al..

arXiv:1111.3119 [physics.ins-det]

1600. "Quasi-Elastic Scattering in MINERvA"

K. S. McFarland [MINERvA Collaboration].

arXiv:1108.0702 [hep-ex]

AIP Conf. Proc. 1405, 95 (2011)

1601. "Indication of Electron Neutrino Appearance from an Accelerator-produced Offaxis Muon Neutrino Beam"

K. Abe et al. [T2K Collaboration].

arXiv:1106.2822 [hep-ex]

Phys. Rev. Lett. 107, 041801 (2011)

1602. "Neutrino Interactions"

K. S. McFarland.

arXiv:0804.3899 [hep-ex]

1603. "A Review of Target Mass Corrections"

I. Schienbein, V. A. Radescu, G. P. Zeller, M. E. Christy, C. E. Keppel, K. S. McFarland, W. Melnitchouk and F. I. Olness et al.

arXiv:0709.1775 [hep-ph]

J. Phys. G G 35, 053101 (2008)

1604. "The CDF Consumer Server Logger Upgrade"

I. Bizjak, F. D. Snider, D. Zhang, F. S. Chlebana, G. M. Guglielmo, K. S. McFarland, W. K. Sakumoto and R. E. Sarkis et al..

1605. "Finding the charge of the top quark in the dilepton channel"

A. Beretvas et al. [CDF Collaboration].

arXiv:0707.1339 [hep-ex]

1606. "MINERvA: A Dedicated neutrino scattering experiment at NuMI"

K. S. McFarland [MINERvA Collaboration].

physics/0605088

Nucl. Phys. Proc. Suppl. 159, 107 (2006)

1607. "NuInt05 session two summary: New experimental results in neutrino scattering physics"

L. Ludovici, K. S. McFarland, M. Shiozawa and G. P. Zeller.

hep-ph/0603002

Nucl. Phys. Proc. Suppl. 159, 35 (2006)

1608. "The MINERvA experiment at FNAL"

K. S. McFarland.

Eur. Phys. J. A 24S2, 187 (2005). 
1609. "Neutral currents and strangeness of the nucleon from the NuTeV experiment" K. S. McFarland.

Eur. Phys. J. A 24S2, 161 (2005).

1610. "The Level-3 trigger at the CDF experiment at Tevatron Run II"

Y. S. Chung, G. De Lentdecker, S. Demers, B. Y. Han, B. Kilminster, J. Lee, K. S. McFarland and A. Vaiciulis et al..

IEEE Trans. Nucl. Sci. 52, 1212 (2005).

1611. "Z' generation with PYTHIA"

C. Ciobanu et al. [CDF Collaboration].

1612. "Measurement of Z-quark, Z-electron couplings and $\sin *^{*} 2$ theta(W) at CDF" G. De Lentdecker et al. [CDF Collaboration].

hep-ex/0409020

eConf C 0406271, MONP07 (2004)

1613. "Electroweak physics at NuTeV"

K. S. McFarland [NuTeV Collaboration].

AIP Conf. Proc. 655, 68 (2003).

1614. "Conventional physics explanations for the NuTeV $\sin ^{* *} 2 \operatorname{theta}(\mathrm{W})$ "

K. S. McFarland and S. -O. Moch.

hep-ph/0306052

1615. "Summary of working group 2"

B. Autin, D. A. Harris, S. F. King, K. S. McFarland and O. Yasuda.

hep-ph/0302218

J. Phys. G G 29, 1743 (2003)

1616. "E1 Working Group summary: Neutrino factories and muon colliders"

T. Adams, C. H. Albright, V. Balbekov, G. Barenboim, D. A. Harris, W. Chou, F. DeJongh and S. Geer et al..

hep-ph/0111030

eConf C 010630, E1001 (2001)

1617. "Young physicists' forum"

T. Adams, M. Bishai, K. A. Bloom, V. Boisvert, L. Coney, R. D. Erbacher, B. T. Fleming and J. Formaggio et al..

hep-ex/0110027

eConf C 010630, I003 (2001)

1618. "Neutrino scattering in a magnetic field"

K. S. McFarland, A. C. Melissinos, N. V. Mikheev and W. W. Repko. hep-ph/0106196

1619. "The Potential for neutrino physics at muon colliders and dedicated high current muon storage rings"

I. I. Y. Bigi, T. Bolton, J. Formaggio, D. A. Harris, B. Kayser, B. J. King, K. S. McFarland 
and J. Morfin et al. .

hep-ph/0106177

Phys. Rept. 371, 151 (2002)

1620. "Oscillation measurements with upgraded conventional neutrino beams"

V. D. Barger, R. Bernstein, A. Bueno, M. Campanelli, D. Casper, F. DeJohgh, S. Geer and M. Goodman et al..

hep-ph/0103052

1621. "Summary of Working Group 2 at NuFact'00"

K. S. McFarland, M. L. Mangano and N. Sasao.

Nucl. Instrum. Meth. A 472, 427 (2000).

1622. "The CDF Run II event data model"

R. D. Kennedy et al. [CDF Collaboration].

1623. "Short-baseline opportunities and challenges at a neutrino factory"

K. S. McFarland.

Nucl. Instrum. Meth. A 451, 218 (2000).

1624. "Consumer-server/logger system for the CDF experiment"

M. Shimojima, B. J. Kilminster, K. S. McFarland, A. Vaiciulis and D. J. Holmgren.

IEEE Trans. Nucl. Sci. 47, 236 (2000).

1625. "Flavor decomposition of nucleon structure at a neutrino factory"

R. D. Ball, D. A. Harris and K. S. McFarland.

hep-ph/0009223

1626. "The neutrino factory: Beam and experiments"

A. Blondel, A. Bueno, M. Campanelli, A. Cervera, D. B. Cline, J. Collot, M. de Jong and

A. Donini et al.

Nucl. Instrum. Meth. A 451, 102 (2000).

1627. "Neutrino mass and oscillation"

P. Fisher, B. Kayser and K. S. McFarland.

hep-ph/9906244

Ann. Rev. Nucl. Part. Sci. 49, 481 (1999)

1628. "Application of PC's and Linux to the CDF Run II level 3 trigger"

J. Fromm, D. J. Holmgren, R. Kennedy, J. Patrick, D. Petravick, R. Rechenmacher, G. P. Yeh and G. Bauer et al..

1629. "ATM based event building and PC based level three trigger at CDF" J. Fromm et al. [CDF Collaboration].

1630. "Search for the lepton-family number violating decays $\mathbf{K}(\mathbf{L})-i$ pi0 mu+- e-+" K. Arisaka, D. Roberts, W. Slater, M. Spencer, M. Weaver, P. Krolak, R. A. Briere and E. Cheu et al.

Phys. Lett. B 432, 230 (1998). 
1631. "NuTeV SSQT performance"

J. Yu et al. [NuTeV Collaboration].

1632. "Deep inelastic scattering at a muon collider: Neutrino physics"

H. Schellman, A. Caldwell, L. de Barbaro, D. Harris, B. King, M. Klasen, A. Kotwal and D. Krakauer et al..

AIP Conf. Proc. 435, 166 (1998).

1633. "A Small target neutrino deep inelastic scattering experiment at the first muon collider"

D. A. Harris and K. S. McFarland.

hep-ex/9804010

AIP Conf. Proc. 435, 505 (1998)

1634. "Detectors for neutrino physics at the first muon collider"

D. A. Harris and K. S. McFarland.

hep-ex/9804009

AIP Conf. Proc. 435, 376 (1998)

1635. "Measurement of the forward-backward charge asymmetry in top quark production in proton-antiproton collisions at 1.96-TeV"

R. Demina.

PoS EPS -HEP2011, 379 (2011).

1636. "Silicon detectors for the sLHC"

A. Affolder, A. Aleev, P. P. Allport, L. Andricek, M. Artuso, J. P. Balbuena, L. Barabash and T. Barber et al..

Nucl. Instrum. Meth. A 658, 11 (2011).

1637. "Track Reconstruction with Cosmic Ray Data at the Tracker Integration Facility"

W. Adam et al. [CMS Collaboration].

1638. "Commissioning and performance of the CMS silicon microstrip detector"

R. Demina [CMS Collaboration].

PoS RD 09, 006 (2009).

1639. "Czochralski Silicon as a Detector Material for S-LHC Tracker Volumes"

L. Spiegel, T. Barvich, B. Betchart, S. Bhattacharya, S. Czellar, R. Demina, A. Dierlamm and M. Frey et al..

arXiv:1008.4107 [physics.ins-det]

Nucl. Instrum. Meth. A 628, 242 (2011)

1640. "The Do Silicon Microstrip Tracker"

S. N. Ahmed et al. [D0 Collaboration].

arXiv:1005.0801 [physics.ins-det]

Nucl. Instrum. Meth. A 634, 8 (2011) 
1641. "TCT and test beam results of irradiated magnetic Czochralski silicon (MCz-Si) detectors"

P. Luukka, J. Harkonen, T. Maenpaa, B. Betchart, S. Czellar, R. Demina, A. Furgeri and Y. Gotra et al..

Nucl. Instrum. Meth. A 604, 254 (2009).

1642. "Measurements on irradiated L1 sensor prototypes for the D0 Run IIb silicon detector project"

M. Ahsan, T. Bolton, K. Carnes, M. Demarteau, R. Demina, T. Gray, S. Korjenevski and F. Lehner et al..

1643. "Test beam results of heavily irradiated magnetic Czochralski silicon (MCz-Si) strip detectors"

P. Luukka, J. Harkonen, T. Maenpaa, B. Betchart, S. Bhattacharya, S. Czellar, R. Demina and A. Dierlamm et al..

Nucl. Instrum. Meth. A 612, 497 (2010).

1644. "Top quark properties and interactions"

R. Demina and E. J. Thomson.

Ann. Rev. Nucl. Part. Sci. 58, 125 (2008).

1645. "Alignment of the CMS Silicon Strip Tracker during stand-alone Commissioning" W. Adam et al. [CMS Collaboration].

arXiv:0904.1220 [physics.ins-det]

JINST 4, T07001 (2009)

1646. "Stand-alone Cosmic Muon Reconstruction Before Installation of the CMS Silicon Strip Tracker"

W. Adam et al. [CMS Tracker Collaboration].

arXiv:0902.1860 [physics.ins-det]

JINST 4, P05004 (2009)

1647. "Performance studies of the CMS Strip Tracker before installation"

W. Adam et al. [CMS Tracker Collaboration].

arXiv:0901.4316 [physics.ins-det]

JINST 4, P06009 (2009)

1648. "Silicon beam telescope for LHC upgrade tests"

T. Maenpaa, P. Luukka, B. Betchart, S. Czellar, R. Demina, Y. Gotra, M. Frey and F. Hartmann et al..

Nucl. Instrum. Meth. A 593, 523 (2008).

1649. "The CMS tracker operation and performance at the Magnet Test and Cosmic Challenge"

W. Adam, T. Bergauer, M. Dragicevic, M. Friedl, R. Fruhwirth, S. Hansel, J. Hrubec and M. Krammer et al..

JINST 3, P07006 (2008). 
1650. "Tracker operation and performance at the Magnet Test and Cosmic Challenge" D. Abbaneo, S. Albergo, F. Ambroglini, P. Azzi, F. Bogelsbacher, N. Bacchetta, R. Bainbridge and T. Barvich et al..

1651. "RD50 status report 2006: Radiation hard semiconductor devices for very high luminosity colliders"

P. Balbuena et al. [RD50 Collaboration].

1652. "Combination of CDF and Do Results on the Mass of the Top Quark"

E. Brubaker et al. [Tevatron Electroweak Working Group Collaboration]. hep-ex/0608032

1653. "Combination of CDF and D0 results on the mass of the top quark" E. Brubaker et al. [Tevatron Electroweak Working Group and CDF and D0 Collaborations]. hep-ex/0603039

1654. "RD50 status report 2005: Radiation hard semiconductor devices for very high luminosity colliders"

F. Campabadal et al. [RD50 Collaboration].

1655. "Calorimeter cell energy thresholds for jet reconstruction in CMS"

R. Demina, J. Dolen, C. Justus, P. Tipton, M. Zielinski, A. Bhatti and R. Harris.

1656. "Development of radiation tolerant semiconductor detectors for the Super-LHC" M. Moll et al. [CERN RD50 Collaboration].

Nucl. Instrum. Meth. A 546, 99 (2005).

1657. "The effect of highly ionising particles on the CMS silicon strip tracker" W. Adam et al. [CMS Tracker Collaboration].

Nucl. Instrum. Meth. A 543, 463 (2005).

1658. "Combination of CDF and D0 results on the top-quark mass"

J. F. Arguin et al. [CDF and D0 and Tevatron Electroweak Working Group Collaboration]. hep-ex/0507091

1659. "Combination of CDF and D0 results on the top-quark mass"

J. F. Arguin et al. [CDF and D0 and Tevatron Electroweak Working Group Collaborations]. hep-ex/0507006

1660. "Prospects for Higgs discovery at LHC"

R. Demina.

AIP Conf. Proc. 753, 343 (2005).

1661. "Supersymmetry searches at the Tevatron"

R. Demina [CDF and D0 Collaboration].

hep-ex/0407001

1662. "eA pion production at CLAS aimed at neutrinos"

S. Manly.

AIP Conf. Proc. 1405, 21 (2011). 
1663. "Neutrino cross sections with the MINERvA experiment"

S. Manly [MINERvA Collaboration].

Nucl. Phys. Proc. Suppl. 217, 211 (2011).

1664. "SiD Letter of Intent"

H. Aihara, (Ed.), P. Burrows, (Ed.), M. Oreglia, (Ed.), E. L. Berger, V. Guarino, J. Repond, H. Weerts and L. Xia et al..

arXiv:0911.0006 [physics.ins-det]

1665. "ILC Reference Design Report Volume 4 - Detectors"

T. Behnke, (Ed.) et al. [ILC Collaboration].

arXiv:0712.2356 [physics.ins-det]

1666. "ILC Reference Design Report: ILC Global Design Effort and World Wide Study"

J. Brau, (Ed.) et al. [ILC Collaboration].

arXiv:0712.1950 [physics.acc-ph]

1667. "International Linear Collider Reference Design Report Volume 2: Physics At The Ilc"

G. Aarons et al. [ILC Collaboration].

arXiv:0709.1893 [hep-ph]

\section{Publications by Rochester Theory group: A. Das, C. R. Hagen, S. Okubo, L. H. Orr, and S. G. Rajeev}

A. Das

1. Tests of general relativity in the transverse space of $p$-branes, A. Das, L. Greenwood and S. Panda (in preparation).

2. Infrared anomaly at finite temperature, A. Das and J. Frenkel, Physics Letters B 696, 556 (2011).

3. Generalization of the Cooper pairing mechanism for spin-triplet in superconductors, A. Das, J. Gamboa, F. Méndez and F. Torres, to be published in Physics Letters A375, 1756 (2011).

4. Finite temperature effective actions, A. Das, XIV Mexican School of Particles and Fields, Journal of Physics: Conference series 287, 012006 (2011).

5. Pseudo-Hermitian quantum mechanics, A. Das, XIV Mexican School of Particles and Fields, Journal of Physics: Conference series 287, 012002 (2011).

6. Phenomenological implications of $S$ duality symmetry, A. Das and J. Maharana, Physics Letters B699, 264 (2011).

7. Aharonov-Bohm effect in a class of noncommutative theories, A. Das, H. Falomir, J. Gamboa, F. Méndez and M. Nieto, Physical Review D84, 045002 (2011). 
8. The thermal chiral anomaly in the Schwinger model, A. Das and J. Frenkel, Physics Letters B704, 85 (2011).

9. Darboux transformation and multi-soliton solutions of the two boson hierarchy, A. Das and U. Saleem, Modern Physics Letters A26, 625 (2011).

10. Generating traveling wave solutions, D. Bazeia, A. Das, L. Losano and M. Santos, Applied Mathematics Letters 23, 681 (2010).

11. An alternative construction of the positive inner product for pseudo-Hermitian Hamiltonians: Examples, A. Das and L. Greenwood, Journal of Mathematical Physics 51, 042103 (2010).

12. The response of laser interferometers to a gravitational wave, A. Melissinos and A. Das, American Journal of Physics 78, 1160 (2010).

13. Thermal effective action for $1+1$ dimensional massive QED, A. Das and J. Frenkel, Physical Review D82, 125002 (2010).

14. Non-commutative supersymmetric quantum mechanics, A. Das, H. Falomir, J. Gamboa and F. Méndez, Physics Letters B670, 407 (2009).

15. Proper acceleration, geometric tachyon and dynamics of a fundamental string near $\mathrm{D} p$ branes, A. Das, S. Panda and S. Roy, Classical and Quantum Gravity 26, 055004 (2009).

16. The structure of supersymmetry in $\mathcal{P} \mathcal{T}$ symmetric quantum mechanics, D. Bazeia, A. Das, L. Greenwood and L. Losano, Physics Letters B673, 283 (2009) .

17. An alternative construction of the positive inner product in non-Hermitian quantum mechanics, A. Das and L. Greenwood, Physics Letters B678, 504 (2009).

18. Finite temperature effective actions, A. Das and J. Frenkel, Physics Letters B680, 195 (2009).

19. Effective actions at finite temperature, A. Das and J. Frenkel, Physical Review D 80, 125039 (2009).

20. $V$ - $A$ theory: A view from the outside, A. Das, J. Phys.:Conf Ser. 196, 012004 (2009).

21. A simple and direct method for generating travelling wave solutions for nonlinear integrable equations, D. Bezeia, A. Das, L. Losano and A. Silva, Annals of Physics 323, 1150 (2008).

22. Origin of the geometric tachyon, A. Das, S. Panda and S. Roy, Physical Review D78, 061901 (2008).

23. Thermal instability in a gravity-like scalar theory, F. T. Brandt, A. Das and J. Frenkel, Physical Review D78, 065030 (2008).

24. "The S-matrix of the Faddeev-Reshetikhin Model, Diagonalizability and PT Symmetry"

A. K. Das, A. Melikyan and V. O. Rivelles.

arXiv:0707.0511 [hep-th]

JHEP 0709, 104 (2007) 
25. "Thermal operator and dispersion relation in QED at finite temperature and chemical potential"

A. K. Das and J. Frenkel.

arXiv:0705.2534 [hep-th]

Phys. Rev. D 76, 087701 (2007)

26. "A Simple and direct method for generating travelling wave solutions for nonlinear integrable equations"

D. Bazeia, A. K. Das, L. Losano and A. Silva.

nlin/0703035

Annals Phys. 323, 1150 (2008)

27. "Hard thermal effective action in QCD through the thermal operator"

A. K. Das and J. Frenkel.

hep-th/0703079 [HEP-TH]

Phys. Rev. D 76, 025009 (2007)

28. "Hard thermal effective actions in the Schwinger formulation"

A. K. Das and J. Frenkel.

hep-th/0612025

Phys. Rev. D 75, 025021 (2007)

29. "CPT / Lorentz Invariance Violation and Quantum Field Theory"

P. Arias, P. Arias, A. K. Das, J. Lopez-Sarrion and F. Mendez.

hep-th/0610152

PoS IC 2006, 022 (2006)

30. "Forward scattering amplitudes and the thermal operator representation"

F. T. Brandt, A. K. Das, J. Frenkel and S. Perez.

hep-th/0609156

Phys. Rev. D 74, 125005 (2006)

31. "CPT / Lorentz invariance violation and neutrino oscillation"

P. Arias, J. Gamboa, J. Lopez-Sarrion, F. Mendez and A. K. Das.

hep-ph/0608007

Phys. Lett. B 650, 401 (2007)

32. "Thermal Operator and Cutting Rules at Finite Temperature and Chemical Potential"

F. T. Brandt, A. K. Das, O. Espinosa, J. Frenkel and S. Perez.

hep-th/0607196

Phys. Rev. D 74, 085006 (2006)

33. "Large-order Perturbation Theory and de Sitter/Anti de Sitter Effective Actions"

A. K. Das and G. V. Dunne.

hep-th/0607168

Phys. Rev. D 74, 044029 (2006) 
34. "Physics of quantum relativity through a linear realization"

A. K. Das and O. C. W. Kong.

gr-qc/0603114

Phys. Rev. D 73, 124029 (2006)

35. "Factorization of finite temperature graphs in thermal QED"

F. T. Brandt, A. K. Das, O. Espinosa, J. Frenkel and S. Perez.

hep-th/0601227

Phys. Rev. D 73, 067702 (2006)

36. "Thermal operator representation of finite temperature graphs. II."

F. T. Brandt, A. K. Das, O. Espinosa, J. Frenkel and S. Perez.

hep-th/0601224

Phys. Rev. D 73, 065010 (2006)

37. "Gauge field theory in the infrared regime"

A. K. Das, J. Gamboa, J. Lopez-Sarrion and F. A. Schaposnik.

hep-th/0510002

Phys. Rev. D 72, 107702 (2005)

38. "The Algebra of flat currents for the string on AdS(5) x $\mathrm{S}^{* * 5}$ in the light-cone gauge"

A. K. Das, A. Melikyan and M. Sato.

hep-th/0508183

JHEP 0511, 015 (2005)

39. "Thermal operator representation of finite temperature graphs"

F. T. Brandt, A. K. Das, O. Espinosa, J. Frenkel and S. Perez.

hep-th/0508067

Phys. Rev. D 72, 085006 (2005)

40. "Supersymmetric non-local gas equation"

A. K. Das and Z. Popowicz.

nlin/0504043 [nlin-si]

J. Math. Phys. 46, 082702 (2005)

41. "Unruh effect in the general light-front frame"

A. K. Das, J. Frenkel and S. Perez.

hep-th/0502243

Phys. Rev. D 71, 105018 (2005)

42. "Propagators with the Mandelstam-Leibbrandt prescription in the light-cone gauge"

A. K. Das and J. Frenkel.

hep-th/0412071

Phys. Rev. D 71, 087701 (2005) 
43. "The Algebra of transition matrices for the $\operatorname{AdS}(5) \times \mathrm{S}^{* * 5}$ superstring" A. K. Das, J. Maharana, A. Melikyan and M. Sato. hep-th/0411200

JHEP 0412, 055 (2004)

44. "Matter-antimatter asymmetry without departure from thermal equilibrium" J. M. Carmona, J. L. Cortes, A. K. Das, J. Gamboa and F. Mendez. hep-th/0410143

Mod. Phys. Lett. A 21, 883 (2006)

45. "Path integral approach to residual gauge fixing" A. K. Das, J. Frenkel and S. Perez. hep-th/0409081 Phys. Rev. D 70, 125001 (2004)

46. "A Nonliearly dispersive fifth order integrable equation and its hierarchy" A. K. Das and Z. Popowicz. nlin/0404049 [nlin-si] J. Nonlin. Math. Phys. 12, 105 (2005)

47. "Quantization in a general light-front frame" A. K. Das and S. Perez. hep-th/0404200 Phys. Rev. D 70, 065006 (2004)

48. "Bosonic reduction of SUSY generalized Harry Dym equation" A. K. Das and Z. Popowicz. nlin/0404017 [nlin-si] J. Phys. A A 37, 8031 (2004)

49. "Monodromy matrix in the PP wave limit"

A. K. Das, J. Maharana and A. Melikyan. hep-th/0402129

Int. J. Mod. Phys. A 19, 4503 (2004)

50. "Chiral bosonization for noncommutative fields"

A. K. Das, J. Gamboa, F. Mendez and J. Lopez-Sarrion. hep-th/0402001

JHEP 0405, 022 (2004)

51. "On a nonlocal gas dynamics like integrable hierarchy"

J. C. Brunelli and A. K. Das.

nlin/0401009 [nlin-si]

52. "Kontsevich product and gauge invariance"

A. K. Das and J. Frenkel.

hep-th/0311243

Phys. Rev. D 69, 065017 (2004) 
53. "Supersymmetric and deformed Harry Dym hierarchies"

J. C. Brunelli, A. K. Das and Z. Popowicz.

hep-th/0311228

54. "Anomalous magnetic moment of electron in Chern-Simons QED"

A. K. Das and S. Perez.

hep-th/0311130

Phys. Lett. B 581, 182 (2004)

55. "Reply to 'Comment on light-front Schwinger model at finite temperature",

A. K. Das and X. -x. Zhou.

hep-th/0311022

Phys. Rev. D 69, 128702 (2004)

56. "Finite temperature field theories on the light front"

A. K. Das.

hep-th/0310247

57. "Background field quantization and noncommutative QED"

A. K. Das, J. Frenkel, S. H. Pereira and J. C. Taylor.

hep-th/0309165

Phys. Lett. B 577, 76 (2003)

58. "Deformed Harry Dym and Hunter-Zheng equations"

J. C. Brunelli, A. K. Das and Z. Popowicz.

nlin/0307043 [nlin-si]

J. Math. Phys. 45, 2646 (2004)

59. "Transport equation for the photon Wigner operator in noncommutative QED" F. T. Brandt, A. K. Das and J. Frenkel.

hep-th/0307010

Phys. Rev. D 68, 085010 (2003)

60. "Light front Schwinger model at finite temperature"

A. K. Das and X. -x. Zhou.

hep-th/0305097

Phys. Rev. D 68, 065017 (2003)

61. "Supersymmetric extensions of the harry dym hierarchy"

J. C. Brunelli, A. K. Das and Z. Popowicz.

nlin/0304047 [nlin-si]

J. Math. Phys. 44, 4756 (2003)

62. "On the energy momentum tensor in noncommutative gauge theories"

A. K. Das and J. Frenkel.

hep-th/0212122

Phys. Rev. D 67, 067701 (2003) 
63. "The Static effective action for noncommutative QED at high temperature" F. T. Brandt, A. K. Das, J. Frenkel, J. Pereira and J. C. Taylor.

hep-th/0212090

Phys. Rev. D 67, 105010 (2003)

64. "Monodromy, duality and integrability of two-dimensional string effective action" A. K. Das, J. Maharana and A. Melikyan.

hep-th/0210012

65. "Light front field theories at finite temperature"

V. S. Alves, A. K. Das and S. Perez.

hep-th/0209036

Phys. Rev. D 66, 125008 (2002)

66. "A Lax equation for the nonlinear sigma model"

J. C. Brunelli, A. Constandache and A. K. Das.

hep-th/0208172

Phys. Lett. B 546, 167 (2002)

67. "Classical transport equation in noncommutative QED at high temperature"

F. T. Brandt, A. K. Das and J. Frenkel.

hep-th/0208115

Phys. Rev. D 66, 105012 (2002)

68. "Comment on 'Supersymmetry in the half oscillator: Revisited",

A. K. Das and S. Pernice.

hep-th/0207112

69. "Dispersion relations for the selfenergy in noncommutative field theories"

F. T. Brandt, A. K. Das and J. Frenkel.

hep-th/0206058

Phys. Rev. D 66, 065017 (2002)

70. "Vanishing magnetic mass in QED(3) with a Chern-Simons term"

A. K. Das and S. Perez.

hep-th/0204259

Phys. Rev. D 66, 025011 (2002)

71. "Alternative dispersionless limit of $\mathbf{N}=\mathbf{2}$ supersymmetric $\mathrm{KdV}$ type hierarchies" A. K. Das, S. Krivonos and Z. Popowicz.

nlin/0204063 [nlin-si]

72. "Generalized Benney lattice and the heavenly equation"

A. Constandache, A. K. Das and Z. Popowicz.

nlin/0204053 [nlin-si]

73. "Transport equation and hard thermal loops in noncommutative Yang-Mills theory"

F. T. Brandt, A. K. Das, J. Frenkel, D. G. C. McKeon and J. C. Taylor. 
hep-th/0204192

Phys. Rev. D 66, 045011 (2002)

74. "A Systematic study of the radion in the compact Randall-Sundrum model" A. K. Das and A. Mitov. hep-th/0203205 Phys. Rev. D 66, 045030 (2002)

75. "Duality, monodromy and integrability of two-dimensional string effective action"

A. K. Das, J. Maharana and A. Melikyan. hep-th/0203144

Phys. Rev. D 65, 126001 (2002)

76. "Screening length in (2+1)-dimensional Abelian Chern-Simons theories" V. S. Alves, A. K. Das and S. Perez. hep-th/0201207

Phys. Lett. B 531, 289 (2002)

77. "General structure of the photon selfenergy in noncommutative QED" F. T. Brandt, A. K. Das and J. Frenkel. hep-th/0112127 Phys. Rev. D 65, 085017 (2002)

78. "Kronecker delta energy terms in thermal field theory" F. T. Brandt, A. K. Das, J. Frenkel and J. C. Taylor. hep-th/0112016 Phys. Rev. D 65, 085008 (2002)

79. "Duality and integrability of two-dimensional string effective action" A. K. Das, J. Maharana and A. Melikyan. hep-th/0111158 Phys. Lett. B 533, 146 (2002)

80. "NonAbelian thermal large gauge transformations in $(2+1)$-dimensions" F. T. Brandt, A. K. Das, G. V. Dunne, J. Frenkel and J. C. Taylor. hep-th/0111146 Phys. Rev. D 65, 065006 (2002)

81. "Parity violating bosonic loops at finite temperature" V. S. Alves, A. K. Das, G. V. Dunne and S. Perez. hep-th/0110160 Phys. Rev. D 65, 085011 (2002)

82. "Selected topics in integrable models"

A. K. Das. hep-th/0110125 
83. "Noncommutativity of boundary closed string coordinates for an open membrane on p-brane"

A. K. Das, J. Maharana and A. Melikyan.

hep-th/0110100

84. "Lucas polynomials and a standard Lax representation for the polytropic gas dynamics"

A. Constandache, A. K. Das and F. Toppan.

hep-th/0110097

Lett. Math. Phys. 60, 197 (2002)

85. "Supersymmetric polytropic gas dynamics"

A. K. Das and Z. Popowicz.

hep-th/0109223

Phys. Lett. A 296, 15 (2002)

86. "Colliding string waves and duality"

A. K. Das, J. Maharana and A. Melikyan.

hep-th/0107229

Phys. Lett. B 518, 306 (2001)

87. "Induced parity violating thermal effective action for $(2+1)$-dimensional fermions interacting with a nonAbelian background"

F. T. Brandt, A. K. Das and J. Frenkel.

hep-th/0107120

Phys. Rev. D 65, 065013 (2002)

88. "Large gauge invariance and finite temperature"

A. K. Das.

hep-th/0104193

89. "Supersymmetric Moyal-Lax representations"

A. K. Das and Z. Popowicz.

hep-th/0104191

90. "Open membranes, p-branes and noncommutativity of boundary string coordinates"

A. K. Das, J. Maharana and A. Melikyan.

hep-th/0103229

JHEP 0104, 016 (2001)

91. "Derivative expansion and the parity violating effective action for thermal (2+1)dimensional QED at higher orders"

F. T. Brandt, A. K. Das, J. Frenkel, S. Pereira and J. C. Taylor.

hep-th/0103221

Phys. Rev. D 64, 065018 (2001) 
92. "Absence of higher order corrections to noncommutative Chern-Simons coupling"

A. K. Das and M. M. Sheikh-Jabbari.

hep-th/0103139

JHEP 0106, 028 (2001)

93. "Properties of Moyal-Lax representation"

A. K. Das and Z. Popowicz.

hep-th/0103063

Phys. Lett. B 510, 264 (2001)

94. "A simpler and elegant algorithm for computing fractal dimension in higher dimensional state space"

S. Ghorui, A. K. Das and N. Venkatramani.

Pramana 54, L331 (2000).

95. "Absence of higher order corrections to the nonAbelian topological mass term" F. T. Brandt, A. K. Das and J. Frenkel.

hep-th/0012087

Phys. Rev. D 63, 085015 (2001)

96. "Probing luminous and dark matter. Proceedings, Symposium in Honor of Adrian Melissinos, Rochester, USA, September 24-25, 1999"

A. K. Das, (ed.) and T. Ferbel, (ed.).

Singapore, Singapore: World Scientific (2000) 290 p

97. "Absence of higher order corrections to the nonAbelian Chern-Simons coefficient"

F. T. Brandt, A. K. Das and J. Frenkel.

hep-th/0009236

Phys. Lett. B 494, 339 (2000)

98. "Behavior of the thermal gluon self energy in the Coulomb gauge"

F. T. Brandt, A. K. Das and J. Frenkel.

hep-th/0009093

Phys. Rev. D 62, 127702 (2000)

99. "Two loop corrections to the topological mass term in thermal QED(3)"

F. T. Brandt, A. K. Das and J. Frenkel.

hep-th/0009031

Phys. Lett. B 492, 393 (2000)

100. "Parity violating electromagnetic interactions in $\operatorname{QED}(3)$ at finite temperature" F. T. Brandt, A. K. Das and J. Frenkel.

hep-ph/0005150

Phys. Rev. D 62, 085012 (2000) 
101. "Supersymmetry in singular quantum mechanics"

A. K. Das.

hep-th/0005042

102. "Finite temperature and large gauge invariance"

A. K. Das.

hep-ph/0005036

Braz. J. Phys. 31, 161 (2001)

103. "New nonlocal charges in SUSY integrable models"

A. K. Das and Z. Popowicz.

nlin/0004034 [nlin-si]

Phys. Lett. A 274, 30 (2000)

104. "Parity breaking electromagnetic interactions in thermal QED(3)"

F. T. Brandt, A. K. Das and J. Frenkel.

hep-ph/0004195

105. "Topics in finite temperature field theory"

A. K. Das.

hep-ph/0004125

In *Mitra, A.N. (ed.): Quantum field theory* 383-411

106. "Large gauge Ward identity"

A. K. Das, G. V. Dunne and J. Frenkel.

hep-th/9911028

Phys. Lett. B 472, 332 (2000)

107. "Dispersionless fermionic KdV"

J. Barcelos-Neto, A. Constandache and A. K. Das.

solv-int/9910001

Phys. Lett. A 268, 342 (2000)

108. "On neutrino masses and mixings from extra dimensions"

A. K. Das and O. C. W. Kong.

hep-ph/9907272

Phys. Lett. B 470, 149 (1999)

109. "Nonstatic dimensional reduction of $\mathrm{QED}(3)$ at finite temperature"

A. K. Das and G. V. Dunne.

hep-th/9905187

Phys. Rev. D 60, 085010 (1999)

110. "Supersymmetry and singular potentials"

A. K. Das and S. A. Pernice.

hep-th/9905135

Nucl. Phys. B 561, 357 (1999) 
111. "Retarded thermal Greens functions and forward scattering amplitudes at two loops"

F. T. Brandt, A. K. Das and J. Frenkel.

hep-th/9902083

Phys. Rev. D 60, 105008 (1999)

112. "A Soluble theory of massless scalar QED(2)"

F. T. Brandt, A. K. Das and J. Frenkel.

hep-th/9810197

Phys. Rev. D 59, 067701 (1999)

113. "Effective actions for $(\mathbf{0}+\mathbf{1})$-dimensional scalar QED and its SUSY generalization at T not equal 0"

J. Barcelos-Neto and A. K. Das.

hep-th/9810065

Phys. Rev. D 59, 087701 (1999)

114. "Retarded Greens functions and forward scattering amplitudes in thermal field theory"

F. T. Brandt, A. K. Das, J. Frenkel and A. J. da Silva.

hep-th $/ 9809177$

Phys. Rev. D 59, 065004 (1999)

115. "Exact effective action for (1+1)-dimensional fermions in an Abelian background at finite temperature"

A. K. Das and A. J. da Silva.

hep-th/9808027

Phys. Rev. D 59, 105011 (1999)

116. "Proper time method for fermions"

A. K. Das and C. Farina.

hep-th/9807152

117. "Derivative expansion and large gauge invariance at finite temperature"

J. Barcelos-Neto and A. K. Das.

hep-th/9805204

Phys. Rev. D 58, 085022 (1998)

118. "Light front Hamiltonian structures for the nonlinear sigma model"

A. K. Das.

hep-th/9802200

Mod. Phys. Lett. A 13, 1133 (1998)

119. "Nonlocal charges and their algebra in topological field theory"

J. C. Brunelli and A. K. Das.

hep-th $/ 9802070$

Phys. Lett. B 438, 99 (1998) 
120. "Finite temperature field theory"

A. K. Das.

Singapore, Singapore: World Scientific (1997) 404 p

121. "Finite temperature perturbation theory and large gauge invariance"

A. K. Das and G. V. Dunne.

hep-th/9712144

Phys. Rev. D 57, 5023 (1998)

122. "Integrable models and the higher dimensional representations of graded Lie algebras"

J. C. Brunelli and A. K. Das.

solv-int/9711001

Mod. Phys. Lett. A 13, 133 (1998)

123. "A Lax representation for the Born-Infeld equation"

J. C. Brunelli and A. K. Das.

hep-th/9712081

Phys. Lett. B 426, 57 (1998)

124. "An SL $(2, \mathrm{Z})$ multiplet of black holes in $\mathrm{D}=4$ type II superstring theory"

A. K. Das, J. Maharana and S. Roy.

hep-th/9709017

Phys. Lett. B 421, 185 (1998)

125. "The muon electric dipole moment"

V. D. Barger, C. Kao and A. K. Das.

hep-ph/9707544

In *Honolulu 1997, B physics and CP violation* 451-456

126. "Supersymmetry and the chiral Schwinger model"

R. Amorim and A. K. Das.

hep-th/9707058

Phys. Rev. D 57, 2599 (1998)

127. "The sTB-B hierarchy"

J. C. Brunelli and A. K. Das.

hep-th/9704126

Phys. Lett. B 409, 229 (1997)

128. "Zero curvature formalism for supersymmetric integrable hierarchies in superspace"

H. Aratyn, A. K. Das and C. Rasinariu.

hep-th/9704119

Mod. Phys. Lett. A 12, 2623 (1997)

129. "Higher dimensional SUSY quantum mechanics"

A. K. Das and S. A. Pernice. 
hep-th/9612125

Mod. Phys. Lett. A 12, 581 (1997)

130. "'Induced' supersymmetry breaking with a vanishing vacuum energy" A. K. Das and S. A. Pernice. hep-th/9612122

Nucl. Phys. B 505, 123 (1997)

131. "The Electric dipole moment of the muon in a two - Higgs doublet model" V. D. Barger, A. K. Das and C. Kao.

hep-ph/9611344

Phys. Rev. D 55, 7099 (1997)

132. "Unidexterous versus ambidexterous gravities"

R. Amorim and A. K. Das.

Phys. Rev. D 54, 4177 (1996).

133. "On M theory and the symmetries of type II string effective actions"

A. K. Das and S. Roy. hep-th/9605073

Nucl. Phys. B 482, 119 (1996)

134. "Cutting rules at finite temperature"

P. F. Bedaque, A. K. Das and S. Naik.

hep-ph/9603325

Mod. Phys. Lett. A 12, 2481 (1997)

135. "On soldering chiralities"

R. Amorim, A. K. Das and C. Wotzasek.

hep-th/9511129

Phys. Rev. D 53, 5810 (1996)

136. "A Two Higgs doublet model for the top quark"

A. K. Das and C. Kao.

hep-ph/9511329

Phys. Lett. B 372, 106 (1996)

137. "The Zero curvature formulation of TB, sTB hierarchy and topological algebras"

A. K. Das and S. Roy.

hep-th/9511091

Mod. Phys. Lett. A 11, 1317 (1996)

138. "Gelfand-Dikii brackets for nonstandard supersymmetric systems"

A. K. Das and S. Panda.

hep-th/9510075

Mod. Phys. Lett. A 11, 723 (1996) 
139. "Introduction to nuclear and particle physics"

A. K. Das and T. Ferbel.

New York, USA: Wiley (1994) 327 p

140. "The Supersymmetric two boson hierarchy"

J. C. Brunelli and A. K. Das.

hep-th/9506096

141. "Supersymmetric two boson equation, its reductions and the nonstandard supersymmetric KP hierarchy"

J. C. Brunelli and A. K. Das.

hep-th/9505093

Int. J. Mod. Phys. A 10, 4563 (1995)

142. "BiHamiltonian structure of the supersymmetric nonlinear Schrodinger equation"

J. C. Brunelli and A. K. Das.

hep-th/9505041

Mod. Phys. Lett. A 10, 2019 (1995)

143. "Thermal effects on the catalysis by a magnetic field"

A. K. Das and M. B. Hott.

hep-th/9504086

Phys. Rev. D 53, 2252 (1996)

144. "Dynamical supersymmetry"

A. K. Das and M. B. Hott.

hep-th/9504059

145. "Properties of nonlocal charges in the supersymmetric two boson hierarchy"

J. C. Brunelli and A. K. Das.

hep-th/9504030

Phys. Lett. B 354, 307 (1995)

146. "Supersymmetric theories on a nonsimply connected space-time"

A. K. Das and M. B. Hott.

hep-th/9502027

Mod. Phys. Lett. A 10, 893 (1995)

147. "Two-dimensional supersymmetric harmonic oscillator carrying a representation of the GL(2/1) graded Lie algebra"

A. K. Das and C. Wotzasek.

hep-th/9501110

J. Math. Phys. 37, 61 (1996)

148. "Properties of an alternate lax description of the KdV hierarchy"

J. C. Brunelli and A. K. Das.

hep-th/9501095

Mod. Phys. Lett. A 10, 931 (1995) 
149. "KdV and NLS equations as triHamiltonian systems"

J. C. Brunelli and A. K. Das.

hep-th/9410165

150. "Path integral solubility of a general two-dimensional model"

A. K. Das and M. B. Hott.

hep-th/9410031

Z. Phys. C 67, 707 (1995)

151. "Duality of the superstring in superspace"

A. K. Das and J. Maharana.

hep-th/9401147

Mod. Phys. Lett. A 9, 1361 (1994)

152. "Field theory: A Path integral approach"

A. K. Das.

World Sci. Lect. Notes Phys. 52, 1 (1993).

153. "On the analytic structure of the selfenergy for massive gauge bosons at finite temperature"

P. B. Arnold, S. Vokos, P. F. Bedaque and A. K. Das.

hep-ph/9211334

Phys. Rev. D 47, 4698 (1993)

154. "Selfduality in $(3+3)$-dimensions and the KP equation"

A. K. Das, E. Sezgin and Z. Khviengia.

hep-th/9206076

Phys. Lett. B 289, 347 (1992)

155. "The superW(infinity) symmetry of the Manin-Radul superKP hierarchy"

A. K. Das, E. Sezgin and S. J. Sin.

hep-th/9111054

Phys. Lett. B 277, 435 (1992)

156. "Temperature dependent anomalous statistics"

A. K. Das and S. Panda.

J. Phys. A A 25, L245 (1992).

157. "Kac-Moody algebras from covariantization of the Lax operators"

A. K. Das and S. Roy.

In *Norman 1990, Proceedings, Beyond the standard model $2 * 360-361$

158. "The Supersymmetric Boussinesq equation"

A. K. Das, W. -J. Huang and S. Roy.

Phys. Lett. A 157, 113 (1991).

159. "Introduction to gauge theories and unification"

A. K. Das. 
In $*$ St. Croix 1990, Proceedings, Techniques and concepts of high-energy physics* 1-47 and Rochester Univ. - UR 1172 (90,rec.Jul.) 56 p

160. "The Zero Curvature Formulation Of The Boussinesq Equation"

A. K. Das, W. -J. Huang and S. Roy.

Phys. Lett. A 163, 186 (1991).

161. "The Origin Of The Q(brst) In Integrable Models"

A. K. Das.

Mod. Phys. Lett. A 5, 1941 (1990).

162. "Integrable Models And Spin Algebras"

A. K. Das and S. Roy.

Int. J. Mod. Phys. A 6, 1429 (1991).

163. "Wormhole solution in coupled Yang-Mills axion system"

A. K. Das and J. Maharana.

Phys. Rev. D 41, 699 (1990).

164. "Observation On The Path Integral Derivation Of The Anomaly"

J. Barcelos-Neto and A. K. Das.

Mod. Phys. Lett. A 5, 2573 (1990).

165. "The Neveu-Schwarz-Ramond String in Background Fields : Nilpotency of BRST Charge"

A. K. Das, J. Maharana and S. Roy.

Nucl. Phys. B 331, 573 (1990).

166. "A Lax description for polytropic gas dynamics"

J. C. Brunelli and A. K. Das.

solv-int/9706005

Phys. Lett. A 235, 597 (1997)

167. "A Note on Abelian conversion of constraints"

R. Amorim and A. K. Das.

hep-th/9408075

Mod. Phys. Lett. A 9, 3543 (1994)

168. "A Nonstandard supersymmetric KP hierarchy"

J. C. Brunelli and A. K. Das.

hep-th/9408049

Rev. Math. Phys. 7, 1181 (1995)

169. "A Superspace formulation of the BV action"

N. R. F. Braga and A. K. Das.

hep-th/9408028

Nucl. Phys. B 442, 655 (1995) 
170. "On the derivative expansion at finite temperature"

A. K. Das and M. B. Hott.

hep-ph/9407283

Phys. Rev. D 50, 6655 (1994)

171. "On the ward identities at finite temperature"

A. K. Das and M. B. Hott.

hep-ph/9406426

Mod. Phys. Lett. A 9, 3383 (1994)

172. "The Supersymmetric two boson hierarchies"

J. C. Brunelli and A. K. Das.

hep-th/9406214

Phys. Lett. B 337, 303 (1994)

173. "Gelfand-Dikii brackets for nonstandard Lax equations"

J. C. Brunelli, A. K. Das and W. -J. Huang.

hep-th/9405111

Mod. Phys. Lett. A 9, 2147 (1994)

174. "Chiral invariance of massive fermions"

A. K. Das and M. B. Hott.

hep-ph/9404317

Mod. Phys. Lett. A 9, 2217 (1994)

175. "Tests of integrability of the supersymmetric nonlinear Schrodinger equation" J. C. Brunelli and A. K. Das.

hep-th/9403019

J. Math. Phys. 36, 268 (1995)

176. "A New class of supersymmetric theories"

A. K. Das.

hep-th/9401138

In *Sudarshan, E.C.G. (ed.): A gift of prophecy* 107-112

177. "Davey-Stewartson equation from a zero curvature and a selfduality condition" J. C. Brunelli and A. K. Das.

hep-th/9312070

Mod. Phys. Lett. A 9, 1267 (1994)

178. "On The higher order corrections to heavy quark effective theory"

A. K. Das. hep-ph/9310372

Mod. Phys. Lett. A 9, 341 (1994)

179. "More on symmetries in heavy quark effective theory"

A. K. Das and V. S. Mathur.

hep-ph/9309306

Phys. Rev. D 49, 2508 (1994) 
180. "Annihilation diagrams in two-body nonleptonic decays of charmed mesons" P. F. Bedaque, A. K. Das and V. S. Mathur.

hep-ph/9309247

Phys. Rev. D 49, 1339 (1994)

181. "Two-body nonleptonic decays of charmed mesons"

P. F. Bedaque, A. K. Das and V. S. Mathur.

hep-ph/9307296

Phys. Rev. D 49, 269 (1994)

182. "Out-of-equilibrium phase transitions and a toy model for disoriented chiral condensates"

P. F. Bedaque and A. K. Das.

hep-ph/9307297

Mod. Phys. Lett. A 8, 3151 (1993)

183. "Nonleptonic decays of charmed mesons into two pseudoscalar mesons"

A. K. Das and V. S. Mathur.

hep-ph/9301279

Mod. Phys. Lett. A 8, 2079 (1993)

184. "Delta expansion and selfconsistent calculation"

P. F. Bedaque and A. K. Das.

hep-th/9211101

185. "Selfduality and the supersymmetric KdV hierarchy"

A. K. Das and C. A. P. Galvao.

hep-th/9211014

Mod. Phys. Lett. A 8, 1399 (1993)

186. "Feynman parametrization and the degenerate electron gas"

P. F. Bedaque and A. K. Das.

hep-ph/9209273

Phys. Rev. D 47, 601 (1993)

187. "Selfduality and the KdV hierarchy"

A. K. Das and C. A. P. Galvao.

hep-th/9209084

Mod. Phys. Lett. A 8, 661 (1993)

188. "The Zero curvature formulation of the KP and sKP equations"

J. Barcelos-Neto, A. K. Das, S. Panda and S. Roy.

Phys. Lett. B 282, 365 (1992).

189. "On the BiHamiltonian structures of the sKdV"

J. Barcelos-Neto and A. K. Das. 
190. "A Note on supersymmetric Gelfand-Dikii brackets"

A. K. Das and W. -J. Huang.

Mod. Phys. Lett. A 7, 2159 (1992).

191. "On the zero momentum limit of Feynman amplitudes at finite temperature"

P. F. Bedaque and A. K. Das.

Phys. Rev. D 45, 2906 (1992).

192. "The Hamiltonian structures associated with a generalized Lax operator"

A. K. Das and W. -J. Huang.

J. Math. Phys. 33, 2487 (1992).

193. "The Hamiltonian structures of the KP hierarchy"

A. K. Das, W. -J. Huang and S. Panda.

Phys. Lett. B 271, 109 (1991).

194. "Zero curvature condition of $\operatorname{OSp}(2 / 2)$ and the associated supergravity theory" A. K. Das, W. -J. Huang and S. Roy.

Int. J. Mod. Phys. A 7, 4293 (1992).

195. "Generalized Schwinger model and a theory of interacting photons and Majorana fermions"

P. F. Bedaque, A. K. Das and W. -J. Huang.

Phys. Rev. D 44, 1818 (1991).

196. "Zero curvature condition and 2-D gravity theories"

A. K. Das, W. -J. Huang and S. Roy.

Int. J. Mod. Phys. A 7, 3447 (1992).

197. "A Geometrical formulation of fermionic integrable systems"

A. K. Das, W. -J. Huang and S. Roy.

J. Math. Phys. 32, 2733 (1991).

198. "Covariant Lax operators and Kac-Moody algebras"

A. K. Das and S. Roy.

J. Math. Phys. 32, 869 (1991).

199. "An Alternate Characterization Of Integrability"

A. K. Das and W. -J. Huang.

J. Math. Phys. 31, 2603 (1990).

200. "Propagators For Shape Invariant Potentials"

A. K. Das and W. -j. Huang.

Phys. Rev. D 41, 3241 (1990).

201. "The Zero Curvature Formulation Of The Skdv Equations"

A. K. Das and S. Roy.

J. Math. Phys. 31, 2145 (1990). 
202. "A Simple Lagrangian For Integrable Systems"

M. A. de Almeida da Silva and A. K. Das.

J. Math. Phys. 31, 798 (1990).

C. R. Hagen

1. "Spin and statistics in Galilean covariant field theory"

C. R. Hagen.

quant-ph/0403039

2. "Casimir effect for the sphere revisited"

C. R. Hagen. quant-ph/0206184

Phys. Lett. A 300, 591 (2002)

3. "Second central extension in Galilean covariant field theory"

C. R. Hagen.

quant-ph/0203109

Phys. Lett. B 539, 168 (2002)

4. "Cutoff dependence of the Casimir effect"

C. R. Hagen.

5. "Cutoff dependence and Lorentz invariance of the Casimir effect"

C. R. Hagen.

quant-ph/0102135

6. "Theoretical high energy physics. Proceedings, 22nd Annual Montreal-RochesterSyracuse-Toronto Conference, MRST'00, Rochester, USA, May 8-9, 2000" C. R. Hagen, (ed.).

7. "Reply to 'Comment on 'Casimir energy for spherical boundaries'",

C. R. Hagen.

hep-th/0004079

8. "Cutoff dependence of the Casimir effect"

C. R. Hagen.

quant-ph/0003108

Eur. Phys. J. C 19, 677 (2001)

9. "Soluble field theory with a massless gauge invariant limit"

C. R. Hagen.

hep-th/9909217

Phys. Rev. D 61, 085004 (2000)

10. "Fractional spin and the Pauli term"

C. R. Hagen.

hep-th/9906172

Phys. Lett. B 470, 119 (1999) 
11. "Casimir energy for spherical boundaries"

C. R. Hagen.

hep-th/9902057

Phys. Rev. D 61, 065005 (2000)

12. "Casimir energy of a spherical shell"

M. E. Bowers and C. R. Hagen.

hep-th/9806193

Phys. Rev. D 59, 025007 (1999)

13. "Nonthermalizability of a quantum field theory"

C. R. Hagen.

hep-th/9802008

14. "Whither the anyon?"

C. R. Hagen.

Z. Naturforsch. A 52, 155 (1997).

15. "Perturbative expansion in the Galilean invariant spin 1/2 Chern-Simons field theory"

C. R. Hagen.

hep-th/9703090

Phys. Rev. D 56, 2250 (1997)

16. "The last of the soluble two dimensional field theories?"

C. R. Hagen.

17. "Comment on 'Quantum phase of induced dipoles moving in a magnetic field", C. R. Hagen. quant-ph/9601001

18. "Comment on 'Statistical mechanics of nonAbelian Chern-Simons particles",

C. R. Hagen.

hep-th $/ 9512076$

Phys. Rev. Lett. 76, 4086 (1996)

19. "Perturbation theory and the Aharonov-Bohm effect"

C. R. Hagen.

hep-th/9503032

Phys. Rev. D 52, 2466 (1995)

20. "Aharonov-Bohm cross-sections as a consequence of symmetry breaking" C. R. Hagen.

In *Sudarshan, E.C.G. (ed.): A gift of prophecy* 218-221

21. "Relativistic Aharonov-Bohm Coulomb problem"

C. R. Hagen and D. K. Park.

hep-th/9410225

J. Korean Phys. Soc. 29, 17 (1996) 
22. "Dimensionality as a perturbation parameter in the generalized hydrogen atom" C. R. Hagen. hep-th/9410161

23. "Unification of the soluble two-dimensional vector coupling models" C. R. Hagen. hep-th/9409038

Phys. Rev. D 55, 1021 (1997)

24. "Comment on 'Anomaly induced magnetic screening in (2+1)-dimensional QED at finite density",

C. R. Hagen.

Phys. Rev. Lett. 73, 1303 (1994).

25. "Irregular solutions in the Aharonov-Bohm scattering of a dirac particle" C. R. Hagen.

26. "Parity conservation in Chern-Simons theories and the anyon interpretation" C. R. Hagen. Phys. Rev. Lett. 68, 3821 (1992).

27. "Comment on 'Berry's phase and persistent charge and spin currents in textured mesoscopic rings."

C. R. Hagen.

28. "Comment on 'Soliton solutions to the gauged nonlinear Schrodinger equation on the plane",

C. R. Hagen.

Phys. Rev. Lett. 66, 2681 (1991).

29. "Transmutation: Fact or fiction?"

C. R. Hagen.

In *Montreal 1990, Proceedings, High energy theory* 163-168.

30. "Comment on 'Statistics transmutation in Maxwell-Chern-Simons theories", C. R. Hagen.

Phys. Rev. D 44, 2614 (1991).

31. "Spin dependence of the Aharonov-Bohm effect: A Review"

C. R. Hagen.

Int. J. Mod. Phys. A 6, 3119 (1991).

32. "Aharonov-Bohm scattering of massive spin one particles"

C. R. Hagen and S. Ramaswamy.

Phys. Rev. D 42, 3524 (1990).

33. "Spin And The Aharonov-bohm Effect"

C. R. Hagen.

In *Columbia 1989, Quantum coherence* $72-81$ 
34. "Exact Equivalence Of Spin 1/2 Aharonov-bohm And Aharonov-casher Effects" C. R. Hagen.

Phys. Rev. Lett. 64, 2347 (1990).

35. "Scaling And Renormalization Of The Vector Meson Field In (2+1) Space" C. R. Hagen and S. Ramaswamy.

Phys. Rev. D 41, 1920 (1990).

36. "Comment On 'gauge Invariance In Chern-simons Theory On A Torus",

C. R. Hagen and E. C. G. Sudarshan.

Phys. Rev. Lett. 64, 1690 (1990).

37. "Flux Carrying Fermions And The Second Virial Coefficient"

T. Blum, C. R. Hagen and S. Ramaswamy.

Phys. Rev. Lett. 64, 709 (1990).

38. "Aharonov-bohm Scattering Of Particles With Spin"

C. R. Hagen.

Phys. Rev. Lett. 64, 503 (1990).

39. "Comment on 'Equivalence of several Chern-Simons matter models",

C. R. Hagen.

hep-th/9404130

40. "Symmetry under alpha - $i$ alpha $+\mathbf{1}$ is forbidden by helicity conservation" C. R. Hagen.

hep-th/9403065

41. "Effects of nongauge potentials on the spin 1/2 Aharonov-Bohm problem"

C. R. Hagen.

hep-th/9308009

Phys. Rev. D 48, 5935 (1993)

42. "Comment on 'Operator algebra in Chern-Simons theory on a torus",

C. R. Hagen and E. C. G. Sudarshan.

hep-th/9303163

Phys. Rev. Lett. 74, 1032 (1995)

43. "Neutron acceleration in uniform electromagnetic fields"

J. Anandan and C. R. Hagen.

hep-th/9301110

44. "Comment on 'attractive forces between electrons in (2+1)-dimensional QED", C. R. Hagen.

hep-th/9212091

Phys. Rev. Lett. 71, 202 (1993)

45. "Comment on 'Gauge independent analysis of Chern-Simons theory with matter coupling", 
C. R. Hagen.

hep-th/9210022

Phys. Rev. Lett. 70, 3518 (1993)

46. "Comment on 'Disassembling anyons",

C. R. Hagen.

hep-th/9209131

47. "Comment on 'Internal frame dragging and a global analog of the AharonovBohm effect",

C. R. Hagen.

hep-th/9209132

48. "The Aharonov-bohm Scattering Amplitude"

C. R. Hagen.

Phys. Rev. D 41, 2015 (1990).

\section{S. Okubo}

1. A. Elduque, S. Okubo, "Special Freudenthal-Kantor triple systems and Lie algebras with dicyclic symmetry", arXiv; 1004.1125 math, Proc. Roy. Soc. Edinburgh 141, 1 (2011).

2. N. Kamiya, D. Mondoc and S. Okubo, "A structure theory of $(-1,-1)$-Freudenthal-Kantor triple systems", Bull. Aust. Math.Soc. 81, 132 (2010).

3. N. Kamiya, D. Mondoc and S. Okubo, "On Lie and Jordan structures associated with $(\epsilon, \delta)$ Freudenthal-Kantor triple systems", Mitt. Math. Ges. Hamburg 29l, 1 (2010).

4. N. Kamiya, D. Mondoc and S. Okubo, " $(\epsilon, \delta)$ Freudenthal-Kantor triple systems, $\delta$-structural Algebras and Lie super algebras", Algebra, Group and Geometry, 27, 191 (2010).

5. N. Kamiya and S. Okubo, "Representation of $(\alpha, \beta, \gamma)$ triple system", Linear Algebra and Multilinear Algebra, 58, 617 (2010).

6. A. Elduque and S. Okubo, "Lie algebras with $S_{3}$ or $S_{4}$-action, and generalized Malcev algebras", Royal Society of Edinburgh, Proceeding A 139A 321 (2009)

7. S. Okubo, "Comments on works of Sudarshan in the 1950's at Rochester", J. Phys. Conf. Ser. 196, 012003 (2009).

8. "Comments on works of Sudarshan in the 1950's at Rochester"

S. Okubo.

J. Phys. Conf. Ser. 196, 012003 (2009).

9. A. Elduque and S. Okubo, " $S_{4}$-symmetry on the TITS construction of exceptional Lie algebras and super Lie algebras", Pub. Math. 52315 (2008)

10. A. Elduque and S. Okubo, "Lie Algebras with $S_{4}$-Action and Structurable Algebras", Journal of Algebra 207, 864 (2007). 
11. "A brief history of the discovery of the SU(3) mass formula and other subjects" S. Okubo.

Mod. Phys. Lett. A 21, 373 (2006).

12. N. Kamiya and S. Okubo, "Composition, Quadratic and Some Triple Systems", Non-associate Algebras and its Applications, ed. by L. Sabinin, L. Sbitneva, and I. Shestakov.

13. S. Okubo, "Algebras Satisfying Symmetric Triality Relations", ibid (as above) 313 (2006).

14. S. Okubo, "Symmetric Triality Relations and Structurable Algebras", Linear Algebras and its Applications 396, 189 (2005).

15. N. Kamiya, A. Elduque, and S. Okubo, "An Reciprocity Relation Between Triple Systems and Binary Algebras", RIMS Kokyuroku 1437, Kyoto University 1 (2005).

16. A. Elduque, N. Kamiya, and S. Okubo, “(-1,-1)-Balanced Freudenthal-Kantor Triple Systems and Non-commutative Jordan Algebras", Journal of Algebras 244, 19 (2005).

17. "Construction of Lie superalgebras from triple product systems"

S. Okubo.

AIP Conf. Proc. 687, 33 (2003).

18. "Gauge theory based upon solvable lie algebras"

S. Okubo.

J. Phys. A A 31, 7603 (1998).

19. "Lorentz invariant Hamiltonian and Riemann hypothesis"

S. Okubo. quant-ph/9707036

J. Phys. A A 31, 1049 (1998)

20. "Jordan-Lie superalgebra and Jordan-Lie triple system"

S. Okubo and N. Kamiya.

21. "Quasiclassical Lie superalgebra and Lie supertriple systems"

S. Okubo and N. Kamiya.

22. "Representation of Clifford algebras and its applications"

S. Okubo. hep-th/9408165

Math. Jap. 41, 59 (1995)

23. "CP violations in $\mathrm{D}+-$ and $\mathrm{B}+-$ boson decays"

S. Okubo.

In *Sudarshan, E.C.G. (ed.): A gift of prophecy* 376-386

24. "Supertriple systems, normal and classical Yang-Baxter equations" S. Okubo. 
25. "Supertriple systems and applications to parastatistics and Yang-Baxter equation"

S. Okubo. hep-th/9306160

In *Syracuse 1993, Proceedings, New directions in the application of symmetry principles to elementary particle physics* 200-213

26. "Yang-Baxter equation and triple product systems"

S. Okubo.

27. "Continuous integrable systems with multipoisson brackets"

S. Okubo.

J. Math. Phys. 33, 2080 (1992).

28. "Existence of gauge field in any partially integrable systems"

S. Okubo.

J. Math. Phys. 33, 2148 (1992).

29. "Poisson brackets and nijenhuis tensor"

S. Okubo.

Z. Naturforsch. A 52, 76 (1997).

30. "Kac-Moody and new infinite dimensional lie algebras"

S. Okubo.

J. Math. Phys. 32, 3333 (1991).

31. "Real representations of Clifford algebras. 2. Explicit construction and pseudooctonion"

S. Okubo.

J. Math. Phys. 32, 1669 (1991).

32. "Some comments on real Clifford algebras"

S. Okubo.

33. "Real representations of finite Clifford algebras. 2. Relation to octonions and $\mathrm{SO}(\mathrm{p}, \mathrm{q}) "$

S. Okubo.

34. "Real representations of finite Clifford algebras. 1. Classification"

S. Okubo.

J. Math. Phys. 32, 1657 (1991).

35. "BRST operator for space with zero curvature but with nonzero torsion tensor" S. Okubo.

Gen. Rel. Grav. 23, 599 (1991).

36. "Introduction to octonion and other nonassociative algebras in physics"

S. Okubo.

Cambridge, UK: Univ. Pr. (1995) 136 p. (Montroll memorial lecture series in mathematical physics, 2) 
37. "Integrability Condition And Finite Periodic Toda Lattice"

S. Okubo.

J. Math. Phys. 31, 1919 (1990).

38. "Some examples of Temperley-Lieb and Hecke algebras"

S. Okubo.

39. "New link invariants and Yang-Baxter equation"

S. Okubo.

hep-th/9407127

40. "Some new solutions of Yang Baxter equation"

S. Okubo.

hep-th/9406087

41. "Parastatistics as Lie supertriple systems"

S. Okubo.

hep-th/9312180

J. Math. Phys. 35, 2785 (1994)

42. "Jordan triple systems and Yang-Baxter equation"

S. Okubo.

hep-th/9312181

43. "Possible tests of CP violations in B+- decays"

S. Okubo.

hep-ph/9308302

44. "Triple products and Yang-Baxter equation. 2. Orthogonal and symplectic ternary systems"

S. Okubo.

hep-th/9212052

J. Math. Phys. 34, 3292 (1993)

45. "Triple products and Yang-Baxter equation. 1. Octonionic and quaternionic triple systems"

S. Okubo.

hep-th/9212051

J. Math. Phys. 34, 3273 (1993)

46. "Argument for the nonexistence of the 'strong CP problem' in QCD"

S. Okubo and R. E. Marshak.

Prog. Theor. Phys. 87, 1059 (1992).

47. "Nijenhuis-Bianchi identify and BRST like operators"

S. Okubo. 
L. H. Orr

1. "Searching for $t \bar{t}$ Resonances at the Large Hadron Collider" U. Baur and L. H. Orr. arXiv:0803.1160 [hep-ph]

Phys. Rev. D 77, 114001 (2008)

2. "ILC Reference Design Report Volume 4 - Detectors" T. Behnke, (Ed.) et al. [ILC Collaboration]. arXiv:0712.2356 [physics.ins-det]

3. "ILC Reference Design Report: ILC Global Design Effort and World Wide Study" J. Brau, (Ed.) et al. [ILC Collaboration]. arXiv:0712.1950 [physics.acc-ph]

4. "International Linear Collider Reference Design Report Volume 2: Physics At The Ilc" G. Aarons et al. [ILC Collaboration]. arXiv:0709.1893 [hep-ph]

5. "High $p_{T}$ Top Quarks at the Large Hadron Collider" U. Baur and L. H. Orr. arXiv:0707.2066 [hep-ph] Phys. Rev. D 76, 094012 (2007)

6. "Probing electroweak top quark couplings at hadron and lepton colliders" U. Baur, A. Juste, L. H. Orr and D. Rainwater. hep-ph/0606264 Nucl. Phys. Proc. Suppl. 160, 17 (2006)

7. "Top at future colliders"

L. H. Orr.

PoS TOP 2006, 031 (2006).

8. "Improved measurement of $t t Z$ couplings at the CERN LHC" U. Baur, A. Juste, D. Rainwater and L. H. Orr. hep-ph/0512262 Phys. Rev. D 73, 034016 (2006)

9. "Probing electroweak top quark couplings at hadron colliders" U. Baur, A. Juste, L. H. Orr and D. Rainwater. hep-ph/0412021 Phys. Rev. D 71, 054013 (2005)

10. "Physics interplay of the LHC and the ILC" G. Weiglein et al. [LHC/LC Study Group Collaboration]. hep-ph/0410364 Phys. Rept. 426, 47 (2006) 
11. "Theoretical progress for the associated production of a Higgs boson with heavy quarks at hadron colliders"

S. Dawson, C. B. Jackson, L. H. Orr, L. Reina and D. Wackeroth.

hep-ph/0311216

Eur. Phys. J. C 33, S451 (2004)

12. "NLO QCD corrections to hadronic Higgs production with heavy quarks" S. Dawson, C. B. Jackson, L. H. Orr, L. Reina and D. Wackeroth. hep-ph/0311105

Nucl. Phys. Proc. Suppl. 133, 111 (2004)

13. "Associated Higgs boson production with heavy quarks"

S. Dawson, L. H. Orr, L. Reina and D. Wackeroth.

hep-ph/0305282

14. "Associated Higgs production with top quarks at the large hadron collider: NLO QCD corrections"

S. Dawson, C. Jackson, L. H. Orr, L. Reina and D. Wackeroth.

hep-ph/0305087

Phys. Rev. D 68, 034022 (2003)

15. "Detecting a Higgs pseudoscalar with a $Z$ boson at the LHC"

C. Kao, G. Lovelace and L. H. Orr.

hep-ph/0305028

Phys. Lett. B 567, 259 (2003)

16. "Associated top quark Higgs boson production at the LHC"

S. Dawson, L. H. Orr, L. Reina and D. Wackeroth.

hep-ph/0211438

Phys. Rev. D 67, 071503 (2003)

17. "NLO QCD predictions for associated $\mathrm{t}$ anti-t $\mathrm{h}$ production in hadronic collisions"

S. Dawson, L. H. Orr, L. Reina and D. Wackeroth.

hep-ph/0211373

Nucl. Phys. Proc. Suppl. 116, 207 (2003)

18. "Direct measurement of the top quark charge at hadron colliders"

U. Baur, M. Buice and L. H. Orr.

hep-ph/0106341

Phys. Rev. D 64, 094019 (2001)

19. "Linear Collider Physics Resource Book for Snowmass 2001 - Part 2: Higgs and Supersymmetry Studies"

T. Abe et al. [American Linear Collider Working Group Collaboration].

hep-ex/0106056

20. "Progress towards a generator for BFKL physics"

L. H. Orr and W. J. Stirling.

hep-ph/0102226 
21. "Top quark physics: Summary"

L. H. Orr.

hep-ph/0102225

22. "QCD loop corrections to e+ e- $-i$ t anti-t $-i b$ W + anti-b W-" C. Macesanu and L. H. Orr.

AIP Conf. Proc. 541, 112 (2000).

23. "BFKL physics in jet production at e+ e- colliders"

L. H. Orr and W. J. Stirling.

hep-ph/0012201

Int. J. Mod. Phys. A 16S1A, 209 (2001)

24. "Top production and decay at linear colliders: QCD corrections"

C. Macesanu and L. H. Orr.

hep-ph/0012200

Int. J. Mod. Phys. A 16S1A, 369 (2001)

25. "Recent results on BFKL physics"

L. H. Orr and W. J. Stirling.

hep-ph/0012198

26. "Gluon radiation in top quark production and decay at e+ e- colliders"

C. Macesanu and L. H. Orr.

hep-ph/0012177

Phys. Rev. D 65, 014004 (2002)

27. "The Case for a 500-GeV e+ e- linear collider"

J. A. Bagger et al. [American Linear Collider Working Group Collaboration].

hep-ex/0007022

28. "Top quark physics"

M. Beneke, I. Efthymiopoulos, M. L. Mangano, J. Womersley, A. Ahmadov, G. Azuelos,

U. Baur and A. Belyaev et al..

hep-ph/0003033

In *Geneva 1999, Standard model physics (and more) at the LHC* 419-529

29. "BFKL Monte Carlo for dijet production at hadron colliders"

L. H. Orr and W. J. Stirling.

hep-ph/0001139

30. "Gluon radiation in top production and decay"

C. Macesanu and L. H. Orr.

hep-ph/0001138

31. "A Monte Carlo for BFKL physics"

L. H. Orr and W. J. Stirling.

hep-ph/0001137 
32. "BFKL dynamics at hadron colliders"

C. Ewerz, L. H. Orr, W. J. Stirling and B. R. Webber.

hep-ph/9912469

J. Phys. G G 26, 696 (2000)

33. "Lepton - chargino mixing and R-parity violating SUSY"

M. Bisset, O. C. W. Kong, C. Macesanu and L. H. Orr.

hep-ph/9907359

In * Ottawa 1999, High energy physics at the millennium* 49-55

34. "Top and gluons at lepton colliders"

C. Macesanu and L. H. Orr.

hep-ph/9906326

35. "R-parity ' $\mathbf{R}$ ' us Rochester"

M. Bisset, O. C. W. Kong, C. Macesanu and L. H. Orr.

In *Montreal 1998, Toward the theory of everything, MRST'98* 254-262

36. "Could the tau be substantially different from e and mu in the supersymmetric standard model?"

M. Bisset, O. C. W. Kong, C. Macesanu and L. H. Orr.

hep-ph/9811499

Nucl. Phys. Proc. Suppl. 76, 201 (1999)

37. "Supersymmetry without R-parity: Leptonic phenomenology"

M. Bisset, O. C. W. Kong, C. Macesanu and L. H. Orr.

hep-ph/9811498

Phys. Rev. D 62, 035001 (2000)

38. "Gluon radiation in top mass reconstruction: Effect of hadronic W decays"

L. H. Orr, T. Andre and T. Stelzer.

hep-ph/9811424

In *Vancouver 1998, High energy physics, vol. 2* 1103-1107

39. "A BFKL Monte Carlo approach to jet production at hadron hadron and lepton - hadron colliders"

L. H. Orr and W. J. Stirling.

hep-ph/9811423

In *Vancouver 1998, High energy physics, vol. $1 * 755-759$

40. "Gluon radiation in top quark production and decay at an e+ e- collider"

C. Macesanu and L. H. Orr.

hep-ph/9808403

In *Montreal 1998, Toward the theory of everything, MRST '98* 28-36

41. "BFKL physics in dijet production at the LHC"

L. H. Orr and W. J. Stirling.

hep-ph/9806371

Phys. Lett. B 436, 372 (1998) 
42. "A BFKL Monte Carlo approach to jet production at hadron hadron and lepton - hadron colliders"

L. H. Orr and W. J. Stirling. hep-ph/9804431

In *Brussels 1998, Deep inelastic scattering and QCD* 553-557

43. "A Simple phenomenological parametrization of supersymmetry without R-parity" M. Bisset, O. C. W. Kong, C. Macesanu and L. H. Orr.

hep-ph/9804282

Phys. Lett. B 430, 274 (1998)

44. "Gluon radiation in top production and decay at lepton colliders"

L. H. Orr.

hep-ph/9802215

In *Batavia 1997, Physics at the first muon collider* 823-830

45. "The Collision energy dependence of dijet cross-sections as a probe of BFKL physics"

L. H. Orr and W. J. Stirling.

hep-ph/9801304

Phys. Lett. B 429, 135 (1998)

46. "Dijet production at hadron hadron colliders in the BFKL approach"

L. H. Orr and W. J. Stirling.

hep-ph/9706529

Phys. Rev. D 56, 5875 (1997)

47. "Top quark physics: Future measurements"

R. Frey, D. W. Gerdes, J. A. Jaros, S. Vejcik, E. L. Berger, R. S. Chivukula, F. Cuypers and P. S. Drell et al..

hep-ph/9704243

eConf C 960625, STC119 (1996)

48. "Gluon radiation and top quark physics"

L. H. Orr, T. Stelzer and W. J. Stirling.

hep-ph/9609354

49. "Gluon radiation in $\mathrm{t}$ anti-t production and decay at the LHC"

L. H. Orr, T. Stelzer and W. J. Stirling.

hep-ph/9609246

Phys. Rev. D 56, 446 (1997)

50. "Soft gluon radiation in top events: Effect of hadronic $W$ decays"

B. Masuda, L. H. Orr and W. J. Stirling.

hep-ph/9605369

Phys. Rev. D 54, 4453 (1996)

51. "On gluon radiation in $t \bar{t}$ production and decay"

L. H. Orr, T. Stelzer and W. J. Stirling. 
52. "Top quark physics: Theory"

L. H. Orr.

53. "On gluon radiation in $t \bar{t}$ production and decay"

L. H. Orr, T. Stelzer and W. J. Stirling.

hep-ph/9505282

Phys. Lett. B 354, 442 (1995)

54. "Gluon radiation in $t \bar{t}$ production at the Tevatron $p \bar{p}$ collider"

L. H. Orr, T. Stelzer and W. J. Stirling.

hep-ph/9412294

Phys. Rev. D 52, 124 (1995)

55. "How to measure $\mathbf{m}(\mathbf{t})$ : A Brief overview"

L. H. Orr.

hep-ph/9409345

In *Meribel les Allues 1994, Proceedings, '94 electroweak interactions and unified theories* 51-58, and Rochester U. - UR-1359 (94/05,rec.Oct.) 9 p

56. "Soft jets and top mass measurement at the Tevatron"

L. H. Orr and W. J. Stirling.

hep-ph/9409365

In *Glasgow 1994, Proceedings, High energy physics, vol. $2 * 835-838$, and Rochester U. UR-1379 (94/08,rec.Oct.) 4 p. Durham U. - DTP-94-072 (94/08,rec.Oct.) 4 p

57. "Top momentum reconstruction and extra soft jets"

L. H. Orr and W. J. Stirling.

hep-ph/9409276

58. "Additional soft jets in $\mathrm{t}$ anti-t production at the tevatron $\mathrm{p}$ anti-p collider"

L. H. Orr and W. J. Stirling.

hep-ph/9409238

Phys. Rev. D 51, 1077 (1995)

59. "Gluon radiation and top width effects"

L. H. Orr, Y. L. Dokshitzer, V. A. Khoze and W. J. Stirling.

hep-ph/9307338

In *Waikoloa 1993, Proceedings, Physics and experiments with linear e+ e- colliders, vol. 2* 670-675. and Calif. Univ. Davis - UCD-93-23 (93/07,rec.Aug.) 6 p. C

60. "Soft photons in W+W-production at LEP-200"

Y. L. Dokshitzer, V. A. Khoze, L. H. Orr and W. J. Stirling.

hep-ph/9305327

Phys. Lett. B 313, 171 (1993)

61. "Properties of soft radiation near $t \bar{t}$ and $W^{-} W^{-}$threshold"

Y. L. Dokshitzer, V. A. Khoze, L. H. Orr and W. J. Stirling.

hep-ph/9302250

Nucl. Phys. B 403, 65 (1993) 
62. "Top width effects in soft gluon radiation"

L. H. Orr, Y. L. Dokshitzer, V. A. Khoze and W. J. Stirling.

hep-ph/9211326

63. "MSSM Higgs bosons at future hadron colliders"

J. F. Gunion and L. H. Orr.

In *Ottawa 1992, Beyond the standard model $3 * 473-477$

64. "Soft gluon radiation in top production and decay"

L. H. Orr, V. A. Khoze and W. J. Stirling.

In *Les Arcs 1992, Perturbative QCD and hadronic interactions* 241-245

65. "Soft gluon radiation in $e+e-\rightarrow \mathbf{t}$ anti-t"

V. A. Khoze, W. J. Stirling and L. H. Orr.

Nucl. Phys. B 378, 413 (1992).

66. "Detecting the Higgs bosons of the minimal supersymmetric model"

J. F. Gunion and L. H. Orr.

Phys. Rev. D 46, 2052 (1992).

67. "Longitudinal W scattering as a probe of a strongly interacting electroweak symmetry breaking sector"

L. H. Orr.

In *Vancouver 1991, Proceedings, Particles and fields '91, vol. 2* 791-795 and Calif. Univ.

Davis - UCD-91-31 (91/09,rec.Dec.) 5 p

68. "Isolating purely leptonic signals for strong $\mathbf{W}$ scattering using antitagging jet tagging, and lepton isolation"

D. A. Dicus, J. F. Gunion, L. H. Orr and R. Vega.

Nucl. Phys. B 377, 31 (1992).

69. "Decay versus hadronization for top quarks produced in hadron colliders"

L. H. Orr.

Phys. Rev. D 44, 88 (1991).

70. "The Longitudinal structure function at HERA: The Effect of next-to-leading order QCD corrections and heavy flavor thresholds"

L. H. Orr and W. J. Stirling.

Phys. Rev. Lett. 66, 1673 (1991).

71. "Comparison Of Top Quark Hadronization And Decay Rates"

L. H. Orr and J. L. Rosner.

Phys. Lett. B 246, 221 (1990).

S. G. Rajeev

1. "A Non-Riemannian Metric on Space-Time Emergent From Scalar Quantum Field Theory"

A. Kar and S. G. Rajeev. arXiv:1207.0748 [hep-th] 
2. "Scalar Quantum Field Theory on Fractals"

A. Kar and S. G. Rajeev.

arXiv:1110.0145 [hep-th]

Annals Phys. 327, 102 (2012)

3. "Global well-posedness and scattering for Skyrme wave maps"

D. -A. Geba, K. Nakanishi and S. G. Rajeev.

arXiv:1106.5750 [math.AP]

4. "A Dispersion Relation for the Density of States With Application to the Casimir Effect"

S. G. Rajeev.

arXiv:1011.4659 [quant-ph]

Annals Phys. 326, 1536 (2011)

5. "On The Relativistic Classical Motion of a Radiating Spinning Particle in a Magnetic Field"

A. Kar and S. G. Rajeev.

arXiv:1010.0221 [hep-th]

Annals Phys. 326, 958 (2011)

6. "The Langevin equation on Lie algebras: Maxwell-Boltzmann is not always the equilibrium"

S. G. Rajeev.

Annals Phys. 324, 2586 (2009).

7. "Nonconcentration of energy for a semilinear Skyrme model"

D. -A. Geba and S. G. Rajeev.

arXiv:1006.3470 [math.AP]

Annals Phys. 325, 2697 (2010)

8. "A Continuity argument for a semilinear Skyrme model"

D. -A. Geba and S. G. Rajeev.

arXiv:1005.1095 [math.AP]

9. "The Geometry of Non-Ideal Fluids"

S. G. Rajeev.

arXiv:1004.2462 [math-ph]

10. "A Lie-Algebraic Approach To the Kondo Problem"

S. G. Rajeev.

arXiv:0911.2858 [math-ph]

Annals Phys. 325, 899 (2010)

11. "The Maxwell-Boltzmann Distribution is not the Equilibrium on a Hyperboloid" S. G. Rajeev.

arXiv:0907.2401 [math-ph] 
12. "Topological Dark Matter in the Little Higgs Models"

A. Joseph and S. G. Rajeev.

arXiv:0905.2772 [hep-ph]

Phys. Rev. D 80, 074009 (2009)

13. "Hamilton-Jacobi formalism for string gas thermodynamics"

A. Joseph and S. G. Rajeev.

arXiv:0807.3957 [hep-th]

Phys. Rev. D 79, 063525 (2009)

14. "A Relativistic Wave Equation for the Skyrmion"

S. G. Rajeev.

arXiv:0801.4538 [hep-th]

Annals Phys. 323, 2873 (2008)

15. "Exact Solution of the Landau-Lifshitz Equations for a Radiating Charged Particle in The Coulomb Potential"

S. G. Rajeev.

arXiv:0801.0238 [hep-th]

Annals Phys. 323, 2654 (2008)

16. "A Hamilton-Jacobi Formalism for Thermodynamics"

S. G. Rajeev.

arXiv:0711.4319 [hep-th]

Annals Phys. 323, 2265 (2008)

17. "Fuzzy fluid mechanics in three dimensions"

S. G. Rajeev.

arXiv:0705.2139 [math-ph]

Int. J. Mod. Phys. A 23, 1019 (2008)

18. "Quantization of Contact Manifolds and Thermodynamics"

S. G. Rajeev. math-ph/0703061 [MATH-PH]

Annals Phys. 323, 768 (2008)

19. "Dissipative Mechanics Using Complex-Valued Hamiltonians"

S. G. Rajeev.

quant-ph/0701141 [QUANT-PH]

20. "Incompressible fluids"

S. G. Rajeev.

Int. J. Mod. Phys. A 20, 6122 (2005).

21. "Yangian symmetries of matrix models and spin chains: The Dilatation operator of $\mathrm{N}=4$ SYM"

A. Agarwal and S. G. Rajeev.

hep-th/0409180

Int. J. Mod. Phys. A 20, 5453 (2005) 
22. "The Dilatation operator of $\mathrm{N}=4 \mathrm{SYM}$ and classical limits of spin chains and matrix models"

A. Agarwal and S. .G. Rajeev.

hep-th/0405116

Mod. Phys. Lett. A 19, 2549 (2004)

23. "Yang-Mills theory on loop space"

S. G. Rajeev.

hep-th/0401215

24. "Three-dimensional simplicial Yang-Mills theory: An Approach to the mass gap" S. G. Rajeev.

hep-th/0401202

25. "Yang-Mills theory on loop space"

S. G. Rajeev.

AIP Conf. Proc. 687, 41 (2003).

26. "Parton distributions from 1+1 QCD"

V. John, G. S. Krishnaswami and S. G. Rajeev.

AIP Conf. Proc. 549, 524 (2000).

27. "New classical limits of quantum theories"

S. G. Rajeev.

hep-th/0210179

28. "Collective potential for large $\mathbf{N}$ Hamiltonian matrix models and free Fisher information"

A. Agarwal, L. Akant, G. S. Krishnaswami and S. G. Rajeev. hep-th/0207200

Int. J. Mod. Phys. A 18, 917 (2003)

29. "A Model of two-dimensional turbulence using random matrix theory"

S. V. Iyer and S. G. Rajeev.

physics/0206083

Mod. Phys. Lett. A 17, 1539 (2002)

30. "A Cohomological interpretation of the Migdal-Makeenko equations"

A. Agarwal and S. G. Rajeev.

hep-th/0202095

Mod. Phys. Lett. A 17, 481 (2002)

31. "Variational principle for large N matrix models"

L. Akant, G. S. Krishnaswami and S. G. Rajeev.

32. "Example of an asymptotically free matrix model"

A. Agarwal and S. G. Rajeev. 
33. "Entropy of operator valued random variables: A Variational principle for large N matrix models"

L. Akant, G. S. Krishnaswami and S. G. Rajeev.

hep-th/0111263

Int. J. Mod. Phys. A 17, 2413 (2002)

34. "Open string field theory of strong interactions in two dimensions"

S. G. Rajeev.

Nucl. Phys. Proc. Suppl. 96, 487 (2001).

35. "Interacting quark model from bi-local soliton picture of baryon in two dimensions"

V. John, G. S. Krishnaswami and S. G. Rajeev.

AIP Conf. Proc. 541, 178 (2000).

36. "A quantum chain model for QCD"

M. N. Coca and S. G. Rajeev.

AIP Conf. Proc. 541, 120 (2000).

37. "Parton model from bilocal solitonic picture of the baryon in two-dimensions" V. John, G. S. Krishnaswami and S. G. Rajeev.

hep-th/0310014

Phys. Lett. B 492, 63 (2000)

38. "An interacting parton model for quark and anti-quark distributions in the baryon"

V. John, G. S. Krishnaswami and S. G. Rajeev.

hep-ph/0310027

Phys. Lett. B 487, 125 (2000)

39. "Parton distribution functions from large N(c) QCD"

S. G. Rajeev.

Nucl. Phys. Proc. Suppl. 86, 86 (2000).

40. "The anti-quark distribution function of the baryon"

V. John, G. S. Krishnaswami and S. G. Rajeev.

hep-ph/9908285

41. "The $\mathrm{x}$ dependence of parton distributions compared with neutrino data"

G. S. Krishnaswami and S. G. Rajeev.

hep-ph/9908279

42. "Valence parton distribution functions from quantum chromodynamics"

S. G. Rajeev.

hep-ph/9908278

43. "Large N Yang-Mills theory as classical mechanics"

C. W. H. Lee and S. G. Rajeev. 
hep-th/9906150

In *Ottawa 1999, High energy physics at the millennium* 72-79

44. "A Review of symmetry algebras of quantum matrix models in the large N limit" C. W. H. Lee and S. G. Rajeev.

hep-th/9906060

Int. J. Mod. Phys. A 14, 4395 (1999)

45. "A Condensation of interacting bosons in two-dimensional space"

S. G. Rajeev.

hep-th/9905120

46. "Derivation of the hadron structure functions from quantum chromodynamics" S. G. Rajeev.

hep-th/9905072

47. "Bound states in models of asymptotic freedom"

S. G. Rajeev.

hep-th/9902025

48. "Symmetries of large-N gauge field theory, M-theory and spin chains" C. W. H. Lee and S. G. Rajeev.

49. "Symmetry of quantum matrix models"

C. W. H. Lee and S. G. Rajeev.

In *Montreal 1998, Toward the theory of everything, MRST'98* 187-194

50. "Poisson brackets of normal ordered Wilson loops"

C. W. H. Lee and S. G. Rajeev.

hep-th $/ 9810233$

J. Math. Phys. 40, 1870 (1999)

51. "A Model of interacting partons for hadronic structure functions"

G. S. Krishnaswami and S. G. Rajeev.

hep-ph/9807345

Phys. Lett. B 441, 429 (1998)

52. "Integrability of supersymmetric quantum matrix models in the large N limit" C. W. H. Lee and S. G. Rajeev.

hep-th/9806019

Phys. Lett. B 436, 91 (1998)

53. "A Lie algebra for closed strings, spin chains and gauge theories"

C. W. H. Lee and S. G. Rajeev.

hep-th/9806002

J. Math. Phys. 39, 5199 (1998)

54. "Symmetry algebras of large N matrix models for open strings" C. W. H. Lee and S. G. Rajeev. 
hep-th/9712090

Nucl. Phys. B 529, 656 (1998)

55. "Symmetries of large N(c) matrix models for closed strings"

C. W. H. Lee and S. G. Rajeev.

hep-th/9711052

Phys. Rev. Lett. 80, 2285 (1998)

56. "Renormalized contact potential in two-dimensions"

R. J. Henderson and S. G. Rajeev.

hep-th/9710061

J. Math. Phys. 39, 749 (1998)

57. "Geometric quantization and two-dimensional QCD"

S. G. Rajeev and O. T. Turgut.

hep-th/9705103

Commun. Math. Phys. 192, 493 (1998)

58. "Renormalized path integral in quantum mechanics"

R. J. Henderson and S. G. Rajeev.

hep-th/9609109

59. "Nontrivial fixed point in four-dimensional scalar field theory and the Higgs mass"

S. G. Rajeev.

hep-th/9607100

60. "Integrability of the Wess-Zumino-Witten model as a nonultralocal theory"

S. G. Rajeev, A. Stern and P. Vitale.

hep-th/9602149

Phys. Lett. B 388, 769 (1996)

61. "Some rigorous results for Yang-Mills theories on a cylinder"

S. G. Rajeev and L. Rossi.

J. Math. Phys. 36, 3308 (1995).

62. "Dynamics of heavy mesons in spherical quantum chromodynamics"

K. S. Gupta, S. Guruswamy and S. G. Rajeev.

In *Syracuse 1993, Proceedings, New directions in the application of symmetry principles to elementary particle physics* $75-87$

63. "Poisson brackets of Wilson loops and derivations of free algebras"

S. G. Rajeev and O. T. Turgut.

hep-th $/ 9508103$

J. Math. Phys. 37, 637 (1996)

64. "Solitons in a bilocal field theory"

R. J. Henderson and S. G. Rajeev. 
hep-th/9501080

Int. J. Mod. Phys. A 10, 3765 (1995)

65. "Yang-Mills theory on a cylinder coupled to point particles"

K. S. Gupta, R. J. Henderson, S. G. Rajeev and O. T. Turgut.

66. "A Three-dimensional conformal field theory"

S. Guruswamy, S. G. Rajeev and P. Vitale.

cond-mat/9408018

In *Montreal 1994, Proceedings, What next?* 58-64, and Rochester U. - UR-1368 (94,rec.Aug.)

$7 \mathrm{p}$

67. "Poisson algebra of Wilson loops in four-dimensional Yang-Mills theory"

S. G. Rajeev and O. T. Turgut.

hep-th/9410053

Int. J. Mod. Phys. A 10, 2479 (1995)

68. " $\mathrm{O}(\mathrm{N})$ sigma model as a three-dimensional conformal field theory"

S. Guruswamy, S. G. Rajeev and P. Vitale.

hep-th/9406010

Nucl. Phys. B 438, 491 (1995)

69. "Alternative canonical formalism for the Wess-Zumino-Witten model"

S. G. Rajeev, G. Sparano and P. Vitale.

hep-th/9312178

Int. J. Mod. Phys. A 9, 5469 (1994)

70. "Symplectic manifolds, coherent states and semiclassical approximation"

S. G. Rajeev, S. Kalyana Rama and S. Sen.

hep-th/9310138

J. Math. Phys. 35, 2259 (1994)

71. "Renormalization in quantum mechanics"

K. S. Gupta and S. G. Rajeev.

hep-th/9305052

Phys. Rev. D 48, 5940 (1993)

72. "Three-dimensional quantum chromodynamics"

G. Ferretti, S. G. Rajeev and Z. Yang.

hep-th/9209129

73. "Two-dimensional meson theory"

S. G. Rajeev.

In *Trieste 1991, Proceedings, High energy physics and cosmology, vol. $2^{*}$ 955-973 and Rochester Univ. - UR 1252 (92/03,rec.Oct.) 19 p

74. "Non-commutative generalizations of Yang-Mills theory"

S. G. Rajeev.

Conf. Proc. C 910725V1, 123 (1991). 
75. "Anomalous scaling of strong interactions at low-energies" S. G. Rajeev.

In *Trieste 1991, Proceedings, High energy physics and cosmology, vol. 2* 1043-1051

76. "Matrix models for Yang-Mills theories"

S. G. Rajeev and G. Ferretti.

In ${ }^{*}$ New York 1991, Proceedings, Differential geometric methods in theoretical physics, vol. $2^{*} 769-779$

77. "Long distance behavior of the four-dimensional nonlinear sigma model with anomaly"

B. E. Baaquie and S. G. Rajeev.

Mod. Phys. Lett. A 8, 625 (1993).

78. "Matrix models with global S0(3) invariance."

G. Ferretti and S. G. Rajeev.

79. "Embedding Yang-Mills theory into universal Yang-Mills theory"

S. G. Rajeev.

Phys. Rev. D 44, 1836 (1991).

80. "Universal Yang-Mills theory and chiral fermions"

J. Mickelsson and S. G. Rajeev.

81. "Deformations of current algebras in (3+1)-dimensions"

J. Mickelsson and S. G. Rajeev.

Lett. Math. Phys. 21, 173 (1991).

82. "What are the anti-particles of $\mathrm{K}(\mathrm{L}, \mathrm{S})$ ?"

V. S. Mathur and S. G. Rajeev.

Mod. Phys. Lett. A 6, 2741 (1991).

83. "UNIVERSAL TEICHMULLER SPACE AND Diff S1 / S1"

D. K. Hong and S. G. Rajeev.

Commun. Math. Phys. 135, 401 (1990).

84. "Universal Gauge Theory"

S. G. Rajeev.

Phys. Rev. D 42, 2779 (1990).

85. "Universal Dirac-yang-mills Theory"

G. Ferretti and S. G. Rajeev.

Phys. Lett. B 244, 265 (1990).

86. "Towards A Bosonization Of Quantum Electrodynamics"

D. K. Hong and S. G. Rajeev.

Phys. Rev. Lett. 64, 2475 (1990). 
87. "The Strong Phase Of Qed"

D. K. Hong and S. G. Rajeev.

Phys. Lett. B 240, 471 (1990).

88. "Quantum gravity on a circle and the diffeomorphism invariance of the Schrodinger equation"

R. J. Henderson and S. G. Rajeev.

gr-qc/9401029

Class. Quant. Grav. 11, 1631 (1994)

89. "Quantum hadrodynamics in two-dimensions"

S. G. Rajeev.

hep-th/9401115

Int. J. Mod. Phys. A 9, 5583 (1994)

90. "Yang-Mills theory on a cylinder coupled to point particles"

K. S. Gupta, R. J. Henderson, S. G. Rajeev and O. T. Turgut.

hep-th/9311064

J. Math. Phys. 35, 3845 (1994)

91. "Spherical quantum chromodynamics of heavy quark systems"

K. S. Gupta, S. Guruswamy and S. G. Rajeev.

hep-ph/9301208

Phys. Rev. D 48, 3354 (1993)

92. "Two-dimensional quantum chromodynamics on a cylinder"

S. Guruswamy and S. G. Rajeev.

hep-th/9210136

Mod. Phys. Lett. A 7, 3783 (1992)

93. "Two-dimensional baryons in the large N limit"

P. F. Bedaque, I. Horvath and S. G. Rajeev.

hep-th/9209027

Mod. Phys. Lett. A 7, 3347 (1992)

94. "Current algebra in three-dimensions"

G. Ferretti and S. G. Rajeev.

hep-th/9207039

Phys. Rev. Lett. 69, 2033 (1992)

95. "The Effective Lagrangian of three-dimensional quantum chromodynamics"

G. Ferretti, S. G. Rajeev and Z. Yang.

hep-th/9204075

Int. J. Mod. Phys. A 7, 7989 (1992)

96. "Baryons as solitons in three-dimensional quantum chromodynamics"

G. Ferretti, S. G. Rajeev and Z. Yang.

hep-th/9204076

Int. J. Mod. Phys. A 7, 8001 (1992) 
97. "CP**(N-1) model with a Chern-Simons term" G. Ferretti and S. G. Rajeev.

hep-th/9202026

Mod. Phys. Lett. A 7, 2087 (1992) 